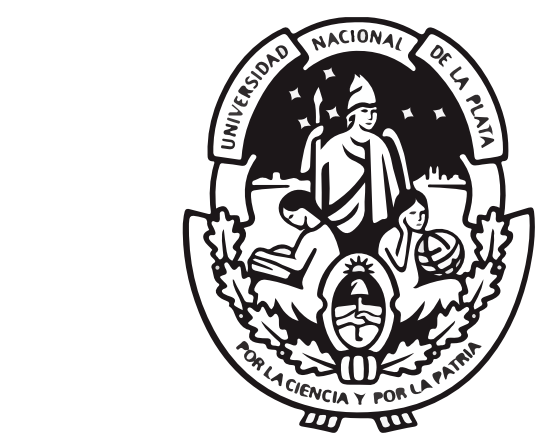

UNIVERSIDAD NACIONAL DE LA PLATA

\title{
MODELADO Y ANÁlisis DE LA RESPUESTA SÍSMICA DE ROCAS HETEROGÉNEAS SATURADAS POR FLUIDOS INMISCIBLES
}

\author{
Santiago G. Solazzi
}

\author{
Director: Dr. Germán Rubino
}

Codirector: Dr. Luis Guarracino

Tesis presentada para obtener el grado académico de Doctor en Geofísica

\author{
La Plata, Argentina \\ - Junio DE 2018 - \\ (C) Santiago G. Solazzi
}


A mi familia 


\section{Resumen}

En el marco de la Geofísica Aplicada y Ambiental, un gran número de actividades involucran la inyección o extracción de fluidos en la corteza terrestre, tales como, la explotación o recuperación secundaria de hidrocarburos, la remediación de acuíferos contaminados, o la captura de $\mathrm{CO}_{2}$ en repositorios geológicos. En este contexto, resulta de vital importancia el desarrollo de nuevas metodologías que permitan caracterizar la distribución espacial de los fluidos porales involucrados y monitorear su evolución temporal. El método sísmico de prospección tiene un gran potencial para lograr este objetivo debido a que las propiedades de las ondas sísmicas pueden ser sensibles a las características de los fluidos porales. En particular, cuando un campo de ondas sísmicas viaja a través de un medio saturado con distribuciones heterogéneas de fluidos inmiscibles, las ondas pueden perder energía debido a un mecanismo conocido como flujo inducido por onda. Este mecanismo de disipación está siendo ampliamente estudiado, ya que un mayor entendimiento del mismo podría permitir una caracterización detallada de la distribución de fluidos y de las propiedades hidromecánicas del subsuelo a partir del dato sísmico. Los modelos empleados para este propósito están basados en la teoría de la poroelasticidad y suelen considerar escenarios donde las rocas se encuentran saturadas por distribuciones binarias de fluidos inmiscibles. Este tipo de distribución de fluidos no responde a las leyes de flujo en medios porosos y, por ello, las firmas sísmicas derivadas pueden conducir a estimaciones erróneas de las propiedades de los medios estudiados. En consecuencia, es preciso generar modelos más realistas para evaluar el impacto y las características de este mecanismo de disipación en rocas parcialmente saturadas.

En el presente trabajo de Tesis se estudia la atenuación y dispersión de las ondas sísmicas por flujo inducido en rocas saturadas por distribuciones realistas de fluidos. Para ello, es necesario modelar adecuadamente tanto la respuesta sísmica como los campos de saturación. Con este objetivo, en primer lugar, se presenta una metodología original para calcular la atenuación por flujo inducido que se encuentra completamente basada en la teoría de la poroelasticidad. Esta metodología permite validar la hipótesis de sólido viscoelástico equivalente, usualmente empleada en la literatura, y brinda herramientas para explorar la contribución local de las diferentes regiones del medio a la atenuación total. En segundo lugar, se estudia la atenuación y dispersión de ondas sísmicas en presencia de campos de saturación realistas. Para ello, se resuelven las ecuaciones de flujo bifásico en medios porosos mediante un método de elementos finitos. Como caso particular de estudio, se analiza la atenuación y dispersión durante un proceso de imbibición. Se observa que las firmas sísmicas son sensibles a los contrastes de compresibilidad generados por el frente de saturación y por ciertas zonas aisladas con alta saturación de agua rodeadas por mezclas de agua y gas. Asimismo, la tasa de inyección del proceso de imbibición afecta significativamente al comportamiento de la atenuación y la velocidad de las ondas sísmicas. Finalmente, se evalúa la capacidad de los modelos binarios de saturación, comúnmente empleados en la literatura, para representar la respuesta sísmica de medios parcialmente saturados. Se demuestra que las estimaciones obtenidas por medio de modelos binarios de saturación no son capaces de representar, de forma simultánea, los niveles de atenuación y dispersión, el nivel de saturación global, y la frecuencia crítica de la muestra saturada de forma realista.

Los aportes realizados en este trabajo de Tesis muestran la importancia de considerar campos de saturación realistas al determinar los efectos de flujo inducido en rocas parcialmente saturadas. En particular, un modelado más realista de los procesos de flujo y de la respuesta sísmica asociada podrían permitir alcanzar algunos de los objetivos centrales de la Geofísica Aplicada y Ambiental, tales como, la determinación de la distribución espacial y de la evolución temporal de los fluidos alojados en el subsuelo. 


\section{Índice}

$\begin{array}{lll}\text { Resumen } & \text { iii }\end{array}$

1. Introducción general 1

1.1. Descripción de los contenidos . . . . . . . . . . . . . 6

2. Ondas sísmicas en medios porosos saturados 9

2.1. Introducción . . . . . . . . . . . . . . . . . . . 9 9

2.2. Teoría de la poroelasticidad de Biot . . . . . . . . . . . . . . 10

2.2.1. Hipótesis . . . . . . . . . . . . . . . . . . . . . . . 10

2.2.2. Variables de la teoría de Biot . . . . . . . . . . . . 11

2.2.3. Densidad de energía elástica de deformación y relaciones constitutivas .......................... 12

2.2.4. Función de disipación . . . . . . . . . . . . . . . . . . . . 14

2.2.5. Densidad de energía cinética . . . . . . . . . . . . 15

2.2.6. Ecuaciones de movimiento . . . . . . . . . . . . . . . 15

2.2.7. Soluciones de las ecuaciones de movimiento . . . . . . . . . . . . 17

2.2.8. Ecuaciones de consolidación de Biot . . . . . . . . . . . . . . . 20

2.3. Flujo inducido por onda en la mesoescala . . . . . . . . . . . . . . . . 21

2.4. Conclusiones . . . . . . . . . . . . . . . . . 23

3. Modelado de atenuación sísmica en medios porosos heterogéneos 24

3.1. Introducción . . . . . . . . . . . . . . . . . . . 24

3.2. Experimentos oscilatorios de relajación . . . . . . . . . . . . 26

3.3. Modelado de la atenuación sísmica utilizando consideraciones energéticas 29

3.3.1. Potencia disipada promedio . . . . . . . . . . . . . . 29

3.3.2. Energía elástica de deformación . . . . . . . . . . . . . . . 30

3.4. Atenuación global y contribución local . . . . . . . . . . . . . . . 31

3.5. Hipótesis de medio viscoelástico equivalente . . . . . . . . . . . . . . . 31

3.6. Modelo 1-D de saturación «patchy» . . . . . . . . . . . . . . . . 32

3.6.1. Medios periódicos estratificados: Teoría de White . . . . . . . 32

3.6.2. Análisis de la atenuación sísmica . . . . . . . . . . . . . . 37

3.7. Modelo estocástico 2-D de saturación «patchy» . . . . . . . . . . . . . 40

3.7.1. Análisis de la atenuación sísmica . . . . . . . . . . . . . . . . 42

3.7.2. Análisis de la energía elástica de deformación . . . . . . . . . . 44

3.8. Modelo 2-D de medio fracturado . . . . . . . . . . . . . . . . 47

3.8.1. Análisis de la atenuación sísmica . . . . . . . . . . . . . . 49

3.9. Conclusiones . . . . . . . . . . . . . . . . . . . . . . 52 
4. Flujo bifásico en medios porosos heterogéneos 53

4.1. Introducción . . . . . . . . . . . . . . . . . 53

4.2. Ecuaciones de flujo bifásico en medios porosos . . . . . . . . 55

4.2.1. Relaciones constitutivas . . . . . . . . . . . . 56

4.3. Formulaciones de las ecuaciones de flujo . . . . . . . . . . . . 59

4.3.1. Formulación de dos presiones . . . . . . . . . . . . . . 59

4.3.2. Formulación fraccional . . . . . . . . . . . . . . . 60

4.3.3. Formulación fraccional alternativa . . . . . . . . . . . . 61

4.4. Problema diferencial . . . . . . . . . . . . . . . . . . . 62

4.5. Discretización temporal . . . . . . . . . . . . . . . . . 62

4.6. Discretización espacial . . . . . . . . . . . . . . . . . 63

4.6.1. Resolución de la ecuación de presión . . . . . . . . . . . 63

4.6.2. Resolución de la ecuación de saturación . . . . . . . . . . . . 66

4.7. Esquema general de la simulación . . . . . . . . . . . . . . 66

4.8. Validación del método numérico . . . . . . . . . . . . . . 67

4.8.1. Solución analítica de la ecuación de Buckley-Leverett . . . . . . 67

4.8.2. Flujo bifásico en medios heterogéneos 2-D . . . . . . . . . 69

4.9. Conclusiones . . . . . . . . . . . . . . . . . . . . . 71

5. Modelado de atenuación y dispersión de ondas sísmicas durante procesos de imbibición

5.1. Introducción . . . . . . . . . . . . . . . . . 72

5.2. Diseño de modelos numéricos 2-D . . . . . . . . . . . . . . 75

5.2.1. Muestra de roca heterogénea y propiedades físicas asociadas . . 75

5.2.2. Modelado del proceso de imbibición . . . . . . . . . . . . 77

5.2.3. Cálculo de atenuación y dispersión sísmica por flujo inducido por onda . . . . . . . . . . . . . . . . . . . 79

5.3. Análisis de la atenuación y dispersión sísmica durante un experimento de imbibición . . . . . . . . . . . . . . . . . 80

5.3.1. Estimación de la atenuación . . . . . . . . . . . . . 80

5.3.2. Estimación de la dispersión sísmica . . . . . . . . . . . . 82

5.3.3. Interpretación física de las curvas de atenuación y dispersión . . 87

5.4. Efecto de la tasa de inyección en la atenuación y dispersión sísmica . . 89

5.5. Discusión de los resultados . . . . . . . . . . . . . . . . . . . . 94

5.6. Conclusiones . . . . . . . . . . . . . . . . . . . . . . . 97

6. Atenuación y dispersión en medios parcialmente saturados: Modelos $\begin{array}{ll}\text { realistas vs. modelos binarios de saturación } & 98\end{array}$

6.1. Introducción . . . . . . . . . . . . . . . . 98

6.2. Respuesta sísmica de una roca homogénea 1-D parcialmente saturada . 101

6.2.1. Muestra de roca y campo de saturación . . . . . . . . . . . . 101

6.2.2. Atenuación y dispersión de las ondas sísmicas . . . . . . . . 102

6.2.3. Contribución local a la atenuación . . . . . . . . . . . . . . 104

6.3. Respuesta sísmica de una roca heterogénea 2-D parcialmente saturada . 105

6.3.1. Muestra de roca y campo de saturación . . . . . . . . . . . 105

6.3.2. Atenuación y dispersión de las ondas sísmicas . . . . . . . . 106

6.3.3. Contribución local a la atenuación . . . . . . . . . . . . 108 
6.4. Limitaciones técnicas y prácticas asociadas al cálculo de los efectos de flujo mesoscópico en medios parcialmente saturados . . . . . . . . . . . 110

6.5. Recomendaciones de aplicación práctica . . . . . . . . . . . . . . . . . 112

6.6. Conclusiones . . . . . . . . . . . . . . . . . . . . . 115

$\begin{array}{ll}\text { 7. Conclusiones } & 116\end{array}$ 
"L'art ne vient pas se coucher dans les lits qu'on a faits pour lui; il se sauve aussitôt qu'on prononce son nom. Ce qu'il aime, c'est l'incognito, ses meilleurs moments sont quand il oublie comment il s'appelle."

Jean Dubuffet

"There is an art to science, and a science in art; the two are not enemies, but different aspects of the whole."

Isaac Asimov 


\section{Capítulo 1}

\section{Introducción general}

La región más somera de la corteza terrestre es una zona de vital importancia para la humanidad, en parte, debido a la vasta cantidad de recursos naturales que se encuentran localizados en las formaciones geológicas que la conforman. Específicamente, los fluidos alojados en el subsuelo, tales como el agua y los hidrocarburos, poseen una significativa importancia socioeconómica. Se estima que un $50 \%$ de la población mundial se abastece de fuentes de agua subterránea (Margat y Van der Gun, 2013; UNESCO, 2003), y que, actualmente, el $56.7 \%$ de la energía empleada por la humanidad proviene del petróleo y el gas (British Petroleum Co., 2016). En consecuencia, la localización, caracterización, y correcta explotación de estos recursos son consideradas actividades primordiales para la sociedad moderna. Los escenarios de interés exploratorio actual presentan complejidades sin precedentes, incluyendo, por ejemplo, la utilización de procesos de inyección y/o extracción de fluidos, tales como, la estimulación hidráulica de reservorios no convencionales, la recuperación secundaria de hidrocarburos, el almacenamiento de $\mathrm{CO}_{2}$ en repositorios geológicos, y la remediación de acuíferos. Es por ello que la comunidad geofísica está abocada al desarrollo de nuevas metodologías que permitan caracterizar la distribución espacial de los fluidos alojados en el subsuelo y su evolución temporal utilizando técnicas no invasivas.

Uno de los métodos geofísicos más utilizados para estudiar las propiedades del subsuelo es el método sísmico de prospección (Lawyer et al., 2001). Este se basa en la generación de ondas sísmicas por medio de fuentes controladas, tales como, la caída de un peso o el uso de un camión vibrador. Las ondas generadas se propagan a través del subsuelo, reflejándose o refractándose en las discontinuidades generadas por las formaciones geológicas. En efecto, debido a reflexiones y refracciones críticas, parte de la energía enviada al subsuelo regresa a la superficie. El tiempo de arribo de las ondas a la superficie puede medirse empleando una serie de instrumentos distribuidos a diferentes distancias de la fuente. Se han creado una serie de estrategias orientadas a convertir los tiempos de arribo a profundidades, permitiendo así generar imágenes de alta calidad de la geometría del subsuelo. Es interesante resaltar que, en su viaje a través del subsuelo, las ondas sísmicas pierden energía, atenuándose. A su vez, el paquete de ondas enviado al subsuelo se dispersa, haciendo posible definir una velocidad de grupo y una velocidad de fase para cada frecuencia. El procesamiento de la información sísmica se realiza, esencialmente, a través de conceptos de elastodinámica (Bourbié et al., 1987). Por lo tanto, la concepción de las rocas del subsuelo como medios porosos saturados no es inherente al método sísmico tradicional.

Más allá de su capacidad para identificar las características geométricas de los horizontes geológicos, el dato sísmico contiene información altamente valiosa respecto del medio explorado. En particular, las ondas sísmicas se ven fuertemente afectadas por 
la presencia de fluidos en los poros de las rocas del subsuelo y por sus propiedades hidráulicas (p. ej., Gassmann, 1951; Pride, 2005; Müller et al., 2010). Evidentemente, si se desea estimar la composición y las propiedades hidráulicas del subsuelo a partir del dato sísmico, es necesario poseer una profunda comprensión del comportamiento hidromecánico de los medios porosos. Los primeros estudios donde se explora la influencia de la porosidad, permeabilidad, y propiedades de los fluidos en la respuesta mecánica de las rocas fueron realizados hace más de 60 años por Terzaghi (Terzaghi, 1943), Frenkel (Frenkel, 1944), Gassmann (Gassmann, 1951), y Biot (Biot, 1941, 1956a,b). El estudio del comportamiento de las rocas a través de esta nueva concepción dio lugar a una reformulación de la teoría de propagación de ondas sísmicas en el subsuelo.

La teoría más aceptada y utilizada para analizar la propagación de ondas sísmicas en medios porosos saturados es conocida como Teoría de la Poroelasticidad o Teoría de Biot (Biot, 1941, 1956a,b, 1962). Esta teoría considera que las rocas están compuestas por una matriz porosa elástica homogénea e isótropa, donde el espacio poral conforma una red interconectada completamente saturada por un fluido viscoso compresible (Biot, 1962). Esencialmente, la teoría de Biot se caracteriza por incluir los efectos de un fluido saturante móvil dentro del espacio poral, lo cual, afecta la física asociada a la propagación de ondas sísmicas. Una de las particularidades más notables de esta formulación es la existencia de un modo de propagación adicional a los modos de propagación clásicos $\mathrm{P}$ y S, presentes en medios elásticos o viscoelásticos, denominado onda lenta compresional (p. ej., Biot, 1956a,b, 1962). La comprobación experimental de la existencia de este modo compresional adicional fue realizada por Plona y Johnson (1980), aproximadamente 25 años después de la publicación de los trabajos de Biot. En el marco de la poroelasticidad, la onda lenta compresional posee un carácter difusivo en el rango de frecuencias de la sísmica de exploración, y es altamente sensible a las propiedades hidráulicas de las rocas. Por ello, los efectos asociados al carácter disipativo de la onda lenta de Biot son ampliamente estudiados tanto a través de modelos numéricos (p. ej., Masson y Pride, 2011; Rubino et al., 2009; Quintal et al., 2011; Müller y Rothert, 2006), como a través de diseños experimentales (p. ej., Chapman et al., 2016; Tisato y Quintal, 2013; Lebedev et al., 2009).

Una forma de analizar los efectos de la presencia de fluidos porales en las ondas sísmicas es a través de mediciones de atenuación y dispersión (p. ej., White, 1975; Dutta y Odé, 1979a,b; Johnson, 2001; Rubino et al., 2009). Estos fenómenos pueden ser causados por efectos elásticos (p. ej., «scattering», divergencia esférica) y por efectos inelásticos, donde la energía de la onda se disipa en forma de calor. Los efectos inelásticos son particularmente interesantes para la sísmica de exploración, ya que las ondas sísmicas pueden sufrir una sustancial pérdida de energía debido a la fricción viscosa asociada al movimiento relativo del fluido poral (Müller et al., 2010). Este tipo de proceso disipativo evidencia una conexión entre observables sísmicos, tales como, la amplitud y la velocidad de las ondas, y propiedades hidráulicas y mecánicas del medio poroso saturado. Sin embargo, estudiar esta conexión no es una tarea sencilla, en parte porque la disipación por fricción viscosa puede responder a diversos procesos físicos, tales como: el mecanismo intrínseco de Biot o flujo global (p. ej., Biot, 1962; Bourbié et al., 1987); el flujo inducido por onda a escala poral o flujo local ${ }^{1}$ (p. ej., Dvorkin et al., 1994, 1995); y el flujo inducido por onda a escala mesoscópica o flujo mesoscópico

${ }^{1} \mathrm{El}$ flujo local es comúnmente denominado «squirt flow» en los textos en inglés. 
(p. ej., Müller et al., 2010; Rubino et al., 2009; Quintal et al., 2011). Cada uno de estos mecanismos de disipación afecta a las ondas sísmicas en un rango de frecuencias característico e involucra procesos que tienen lugar a determinadas escalas espaciales, diferenciadas en la Figura 1.1 en función de la longitud de onda $\lambda$ de una onda sísmica monocromática. En particular, es de nuestro interés estudiar la disipación de las ondas sísmicas en el rango de frecuencias de la sísmica de exploración (incluyendo mediciones «crosshole»y de perfilajes sónicos), es decir, de $1 \mathrm{~Hz}$ a algunos $\mathrm{kHz}$.
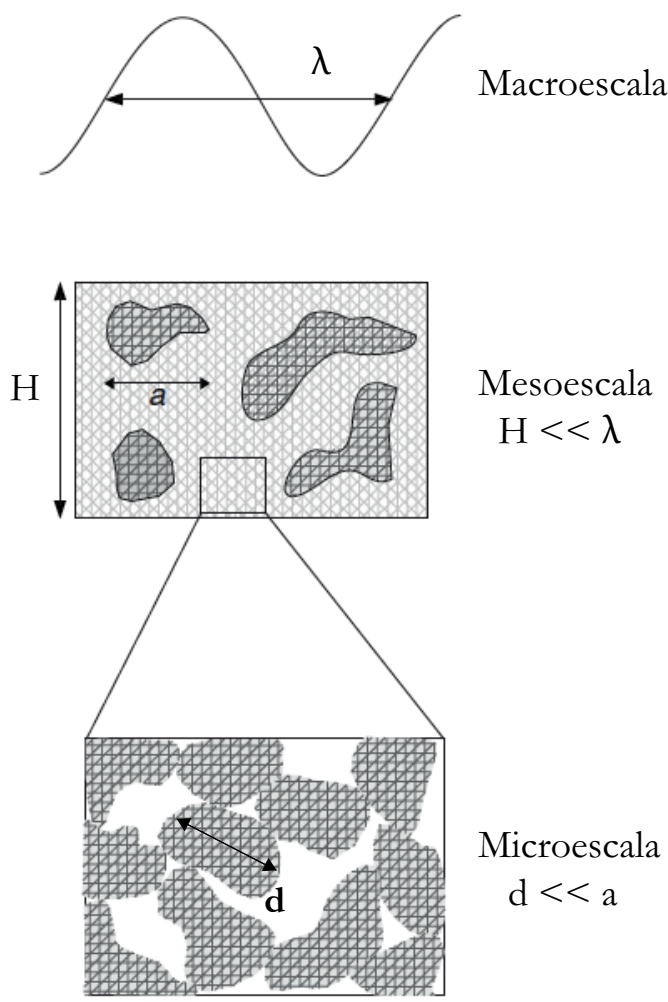

Figura 1.1. Tamaño de las heterogeneidades involucradas en los diversos procesos disipativos (modificado a partir de Müller et al. (2010)).

Cuando un campo de ondas $\mathrm{P}$ o S se propaga por un medio poroelástico homogéneo, las ondas se atenúan por medio del mecanismo intrínseco de Biot. Este mecanismo de disipación puede manifestarse a través de dos fenómenos de relajación diferentes (Pride, 2005). El primero está asociado a la generación de una capa límite viscosa dentro del espacio poral. Debido a las aceleraciones causadas por un campo de onda sísmica clásico compresional y/o de corte, la matriz arrastra parte del fluido poral en su movimiento, generando disipación por fricción viscosa dentro del espacio poral (Biot, 1956a,b; Bourbié et al., 1987). El segundo fenómeno de relajación puede ocurrir solamente en el caso de ondas compresionales, y sucede cuando existe tiempo suficiente en un período de onda para que se equilibren las presiones entre las regiones del medio sujetas a compresión y aquellas sujetas a extensión. Este mecanismo de disipación suele ser también denominado flujo global (Pride, 2005), y tiene lugar, primordialmente, en el rango de frecuencias ultrasónico (p. ej., Müller et al., 2010; Johnston et al., 1979; Winkler, 1985; Pride, 2005). 
Por otro lado, cuando una onda sísmica se propaga a través de un medio que posee heterogeneidades de microescala (ver Figura 1.1, panel inferior), tales como, por ejemplo, microfracturas o contactos entre granos mal cementados, las ondas pierden energía por flujo a escala poral. Debido a las diferencias geométricas y mecánicas de estas microestructuras respecto del espacio poral principal en su entorno inmediato, la deformación producida por el campo de onda genera gradientes de presión. Estos gradientes tienden a relajarse, generando disipación por flujo viscoso (p. ej., Pride et al., 2004). Se considera que la principal contribución a la atenuación y a la dispersión sísmica de este mecanismo ocurre en frecuencias ultrasónicas, aunque también puede afectar a las ondas en las frecuencias sísmicas (p. ej., Müller et al., 2010).

Por último, el flujo inducido por onda a escalas mesoscópicas es considerado un mecanismo de disipación predominante en el rango de frecuencias de la sísmica de exploración (p. ej., Müller et al., 2010). El flujo mesoscópico tiene lugar cuando una onda sísmica se propaga por un medio que contiene heterogeneidades de tamaños mayores que la escala poral típica, pero mucho menores que las longitudes de onda predominantes, como se ilustra en el panel central de la Figura 1.1. Si las heterogeneidades poseen compresibilidades distintas a las de su entorno, el paso de una onda generará gradientes de presión y disipación por flujo viscoso. Puede demostrarse que este proceso no es más que una conversión de la energía de un modo de propagación clásico a ondas lentas de Biot con carácter difusivo en las interfases de las heterogeneidades (Pride et al., 2002). Este mecanismo de disipación fue propuesto por White (1975) y White et al. (1975). Los trabajos de Dutta y Odé (1979a,b) analizaron este proceso físico utilizando la teoría de la poroelasticidad de Biot, dándole así un marco teórico más sólido. Uno de los aspectos más interesantes del flujo inducido por onda en la mesoescala es que el proceso de disipación depende directamente de propiedades hidráulicas de la matriz, es decir, de la permeabilidad del medio, como así también de las propiedades físicas de los fluidos porales, y de factores relacionados con la geometría de las heterogeneidades. Es por ello que el comportamiento de la atenuación y de la dispersión de la velocidad asociadas al proceso de flujo inducido por onda en la mesoescala poseen, potencialmente, la capacidad de proveer información sobre las propiedades hidráulicas de la matriz, el contenido de fluidos, y la geometría de sus distribuciones espaciales.

La interpretación del proceso físico de flujo inducido por onda a escalas mesoscópicas se basa en modelos analíticos (p. ej., White, 1975; Johnson, 2001; Pride et al., 2004) o en modelos numéricos de homogeneización ${ }^{2}$ (p. ej., Masson y Pride, 2007; Rubino et al., 2009; Quintal et al., 2011; Wenzlau et al., 2010). En general, estos modelos hacen uso de la hipótesis de medio viscoelástico equivalente. Es decir, asumen que la roca porosa heterogénea estudiada puede ser representada por un medio homogéneo viscoelástico que exhibe la misma respuesta efectiva en función de la frecuencia que el medio original al ser sujeto a un campo de tensiones o deformaciones idéntico. Los medios viscoelásticos resultantes han sido empleados por algunos investigadores para efectuar simulaciones de propagación de ondas (p. ej., Rubino et al., 2011; Sidler et al., 2013) o para comparar las curvas de atenuación y dispersión con modelos viscoelásticos específicos (p. ej., Picotti et al., 2010). Sin embargo, es de vital importancia contar con una metodología alternativa para calcular la atenuación sísmica, completamente basada en la teoría de la poroelasticidad, para corroborar la validez de esta hipótesis. La

\footnotetext{
${ }^{2}$ La homogeneización suele denominarse «upscaling» en los textos en inglés.
} 
razón principal por la cual es importante estudiar la validez de la hipótesis de medio viscoelástico equivalente es que, más allá de la física que gobierna las ecuaciones a escala local y de las condiciones de borde impuestas, siempre se puede definir un módulo viscoelástico equivalente en términos de promedios volumétricos de los campos de tensión y deformación. Sin embargo, no hay garantías de que estos módulos representen de manera adecuada el comportamiento efectivo del medio heterogéneo original desde el punto de vista de la energía elástica almacenada y de la energía disipada. En el caso de fallar en este tipo de representación, los parámetros sísmicos derivados no serían representativos de los medios que se desean estudiar.

Es importante destacar que, para que las ondas sísmicas sufran disipación por flujo inducido en un medio homogéneo saturado por dos fluidos inmiscibles, el campo de saturación debe presentar heterogeneidades de escalas mesoscópicas y las fases fluidas involucradas deben poseer contrastes de compresibilidad significativos. Debido a que las distribuciones de agua y gas suelen satisfacer esta condición, el estudio de este tipo de escenario, comúnmente denominado ambiente de saturación "patchy», es muy común en la literatura. El término anglosajón «patch» hace referencia a regiones de tamaño mesoscópico saturadas de forma predominante por una de las fases fluidas (p. ej., gas metano o $\mathrm{CO}_{2}$ ) rodeadas de zonas saturadas mayormente por la fase fluida restante (p. ej., agua). Los efectos del flujo mesoscópico en ambientes con saturación «patchy» han sido modelados empleando procedimientos que poseen limitaciones inherentes, como es el caso de los desarrollos analíticos, o han sido utilizados considerando distribuciones de fluidos muy simples. Por ejemplo, la mayoría de los modelos analíticos proponen rocas de matriz homogénea saturadas por «patches» binarios de fluidos (p. ej., White, 1975; White et al., 1975; Johnson, 2001). Los modelos de homogeneización numérica son más flexibles en este sentido, sin embargo, la mayoría de los trabajos suelen considerar «patches» binarios de saturación basados en procesos estocásticos (p. ej., Rubino et al., 2011; Masson y Pride, 2011). Existen razones físicas que permiten cuestionar la existencia de distribuciones binarias de saturación en la mesoescala, tales como, procesos de difusión, o flotabilidad. De hecho, varios estudios experimentales evidencian que los campos de saturación de mesoescala, logrados en muestras de roca a partir de procesos de inyección de fluidos inmiscibles, poseen variaciones espaciales suaves asociadas a las heterogeneidades de la matriz (p. ej., Cadoret et al., 1995; Toms-Stewart et al., 2009; Alemu et al., 2013). Por lo tanto, los resultados derivados de modelos de saturación binarios pueden estar estimando de manera errónea los niveles de atenuación y de dispersión sísmica en rocas saturadas por fluidos inmiscibles. Rubino y Holliger (2012) propusieron, por primera vez en la literatura, estudiar la atenuación y dispersión por flujo mesoscópico conectando el patrón de saturación con las heterogeneidades de la matriz, generando así campos de saturación con variaciones espaciales suaves. Para ello, asumieron que los fluidos saturantes (gas y agua) se encontraban en equilibrio capilar. Sus resultados muestran que los niveles de atenuación y dispersión sísmica calculados discrepan de los resultados obtenidos empleando modelos binarios de saturación. Estos resultados evidencian que es necesario explorar los efectos del flujo inducido por onda utilizando modelos de saturación de fluidos realistas, dejando de lado los modelos binarios de saturación. Una propuesta interesante consiste en generar modelos donde la distribución de fluidos responda a las ecuaciones de flujo en medios porosos. En este contexto, el modelado numérico de flujo bifásico en medios porosos 
constituye una herramienta de gran valor, la cual permite explorar la complejidad de los campos de saturación y su relación con las propiedades del medio y de los fluidos.

Desde un enfoque experimental, se han desarrollado una serie de estrategias de laboratorio que apuntan a relacionar el contenido y la distribución espacial de los fluidos porales con las mediciones de atenuación y dispersión sísmica. La estrategia más utilizada consiste en medir, de manera transitoria, las propiedades sísmicas de una roca saturada por una fase fluida determinada mientras esta es desplazada por una segunda fase (Lopes y Lebedev, 2012; Lopes et al., 2014; Zhang et al., 2015; Alemu et al., 2013). Este tipo de procesos de flujo presentan dos casos extremos, de gran interés teórico y práctico, denominados drenaje e imbibición. Durante un procedimiento de drenaje, un fluido no mojante (p. ej., gas) es inyectado en una roca inicialmente saturada por un fluido mojante (p. ej., agua). De manera inversa, un proceso de imbibición consiste en la inyección de un fluido mojante en una roca inicialmente saturada por un fluido no mojante. Al llevar a cabo alguno de estos procesos, los patrones de saturación de fluido resultantes dependerán de una serie de factores, tales como, las heterogeneidades de la roca, y la acción combinada de fuerzas capilares, viscosas, y gravitacionales (p. ej., Shi et al., 2011). La evidencia experimental indica que los patrones de saturación heterogéneos resultantes pueden producir atenuación y dispersión de las ondas sísmicas (p. ej., Tisato y Quintal, 2013; Yin et al., 1992). Es interesante resaltar que los patrones de fluidos resultantes de procesos de imbibición y de drenaje suelen diferir, evidenciando la existencia de un proceso de histéresis (p. ej., Cadoret et al., 1998; Le Ravalec et al., 1996). Además, se ha observado que la distribución espacial de los fluidos porales es altamente sensible a la velocidad con la que una fase fluida desplaza a la otra (p. ej., Riaz et al., 2007; Lenormand y Zarcone, 1984; Lenormand, 1990). Al cambiar las características del proceso de inyección, no solo cambia la distribución espacial de los fluidos, sino que, además, la respuesta sísmica se ve significativamente afectada, como se ha observado en estudios recientes (Lopes y Lebedev, 2012; Lopes et al., 2014). Estos resultados evidencian una conexión entre las características del proceso de inyección de fluido, la distribución de fluidos resultante, y la respuesta sísmica asociada. Sin embargo, las causas físicas de la atenuación y dispersión de ondas sísmicas observadas en el laboratorio durante procesos de inyección de fluidos y, en particular, su relación con la tasa de inyección, no han sido explorados en profundidad.

En la actualidad, se observa una falta de trabajos en el contexto de flujo inducido por onda a escalas mesoscópicas en medios porosos que contienen patrones de saturación realistas en general, y en caso de saturación transitoria en particular. La evolución temporal de los fluidos saturantes puede ser modelada resolviendo las ecuaciones de flujo bifásico, utilizando condiciones iniciales y de borde adecuadas. Si deseamos comprender el impacto que poseen las distribuciones realistas de fluidos inmiscibles en las propiedades de las ondas sísmicas es necesario realizar un análisis conjunto, considerando procesos de flujo bifásico y cuantificando la disipación sísmica asociada.

\subsection{Descripción de los contenidos}

El principal objetivo de este trabajo de Tesis consiste en analizar los efectos del flujo inducido en la atenuación y dispersión de las ondas sísmicas en medios porosos heterogéneos saturados con patrones realistas de fluidos inmiscibles. Para ello, por un 
lado, debemos contar con herramientas adecuadas que nos permitan generar modelos de roca parcialmente saturada respetando las leyes de flujo bifásico en medios porosos. Por el otro, es necesario poseer una metodología de cálculo de la atenuación sísmica consistente con la formulación empleada, es decir, con la teoría de la poroelasticidad. En conjunto, ambas técnicas brindan la posibilidad de estudiar las relaciones existentes entre las características de los procesos de flujo y las firmas sísmicas de los medios parcialmente saturados resultantes. A su vez, este tipo de análisis conjunto permite evaluar la representatividad de las firmas sísmicas utilizando modelos binarios de saturación, ya que estos son usualmente empleados en la literatura. Con este objetivo, la presentación de los contenidos de este trabajo de Tesis ha sido estructurada en siete Capítulos.

El Capítulo 2 contiene una breve descripción de la teoría de la poroelasticidad de Biot (1962). Se derivan las relaciones constitutivas y las ecuaciones de movimiento para medios poroelásticos por medio de una formulación de medio continuo. Se resuelven las ecuaciones de onda y se describen las propiedades de los modos resultantes de esta teoría. Luego, se presentan las ecuaciones de consolidación de Biot (1941), cuya resolución permite explorar el carácter difusivo de la onda lenta en bajas frecuencias. Finalmente, se introduce el flujo inducido a escalas mesoscópicas como mecanismo de atenuación y dispersión.

Con el fin de evaluar la validez de la hipótesis de medio viscoelástico equivalente, en el Capítulo 3 se propone una metodología original de cálculo de la atenuación sísmica por flujo mesoscópico basada en la teoría de poroelasticidad. Esta nueva metodología utiliza la energía elástica de deformación y la potencia disipada, en respuesta a un test oscilatorio de relajación. A continuación, con la intención de analizar los patrones de disipación de energía, se define la contribución local a la atenuación total. Este parámetro expresa la contribución de las diferentes regiones de la roca saturada al proceso disipativo generado por el paso de una onda sísmica. Finalmente, se evalúa la validez de la hipótesis de medio viscoelástico equivalente, y se estudia la energía elástica almacenada y la contribución local a la atenuación en medios 1-D y 2-D parcialmente saturados con distribuciones binarias de fluidos inmiscibles, y en medios fracturados.

Con la motivación de calcular la atenuación sísmica en medios saturados con distribuciones realistas de fluidos, se propone realizar simulaciones de flujo bifásico en medios porosos heterogéneos. Con este objetivo, en el Capítulo 4 se introducen las ecuaciones de flujo bifásico en medios porosos y las relaciones constitutivas asociadas. Se presenta una formulación de las ecuaciones que permite modelar procesos de flujo en medios porosos heterogéneos. Las ecuaciones en cuestión conforman un sistema altamente no lineal y acoplado. En primer lugar se lleva a cabo una discretización temporal de las ecuaciones empleando un esquema implícito en la presión y explícito en la saturación (IMPES). Luego, se describe un método de elementos finitos mixtos híbridos, combinado con elementos finitos Galerkin discontinuos, que permite resolver el flujo simultáneo de dos fases fluidas inmiscibles e incompresibles en un medio poroso altamente heterogéneo, considerando efectos de capilaridad, gravedad, y permitiendo saltos de saturación. Finalmente, se procede a validar los resultados del método numérico.

En el Capítulo 5 se utilizan los métodos descriptos en los Capítulos 3 y 4 para analizar la respuesta sísmica de rocas heterogéneas durante procesos de imbibición de fluidos. Se analizan los cambios en las curvas de atenuación y dispersión de la velocidad 
para diferentes estadíos del proceso de inyección. A su vez, se utiliza la contribución local a la atenuación para explorar las zonas de la roca parcialmente saturada que contribuyen significativamente al fenómeno de flujo inducido por onda. Por último, se realiza un estudio del efecto que posee la tasa de inyección de fluidos en los patrones de saturación, y en la atenuación y la dispersión de las ondas sísmicas.

En el Capítulo 6, se evalúa la validez de los modelos binarios de saturación, ampliamente utilizados en la literatura, para representar la respuesta sísmica de rocas saturadas por medio de procesos de imbibición. En primer lugar, se analiza el caso de la atenuación y dispersión generada por la presencia de un frente de saturación en una roca homogénea. Se analiza el alcance del modelo de White et al. (1975) para representar los niveles de saturación, atenuación, y dispersión de la roca saturada de forma realista. Luego, se procede a estudiar casos más complejos utilizando una roca heterogénea saturada por medio del proceso de imbibición descripto en el Capítulo 5. Se propone binarizar la distribución de fluidos para un estadío particular, considerando diferentes criterios de binarización. Luego se compara la saturación global, la atenuación y la dispersión resultantes de considerar distribuciones binarias de fluidos con la respuesta sísmica del medio realista asociado.

Finalmente, en el Capítulo 7 se destacan los aportes y contribuciones más importantes resultantes de este trabajo de Tesis. 


\section{Capítulo 2}

\section{Ondas sísmicas en medios porosos sa- turados}

En este Capítulo presentaremos una síntesis de la teoría de la poroelasticidad de Biot. En primer lugar, comentaremos las hipótesis en las que se fundamenta esta teoría, para luego derivar las relaciones constitutivas y las ecuaciones de movimiento. El sistema de ecuaciones resultante permite caracterizar los medios porosos saturados desde un punto de vista sísmico. Luego, resolveremos las ecuaciones de onda asociadas y analizaremos las características de las ondas descriptas por esta teoría. A continuación, introduciremos las ecuaciones de consolidación de Biot, comúnmente empleadas para explorar la disipación de energía causada por procesos de difusión de presión en bajas frecuencias. Finalmente, describiremos las bases físicas del proceso de flujo inducido por onda en la mesoescala en medios porosos heterogéneos. Los contenidos del presente Capítulo son el resultado de una recopilación bibliográfica de textos clásicos y publicaciones actuales. En caso de requerir información más detallada sobre la teoría de la poroelasticidad, referimos al lector a los trabajos de Biot (1941, 1956a,b, 1962) y al libro de Bourbié et al. (1987). Para mayor información respecto del proceso de flujo inducido por onda en la mesoescala, se recomienda la lectura de la revisión llevada a cabo por Müller et al. (2010).

\subsection{Introducción}

Un medio poroso es un material que posee un esqueleto sólido, comúnmente denominado matriz, que posee en su interior espacios vacíos interconectados que conforman el espacio poral. En particular, los medios porosos pueden estar completamente saturados por una fase fluida o poseer una saturación parcial, es decir, que su espacio poral puede albergar más de una fase fluida. Muchos materiales tales como rocas, suelos, tejidos biológicos, cementos, y ciertos alimentos, entre otros, pueden ser representados utilizando medios porosos. En particular las rocas que contienen agua, petróleo, y/o gas pueden describirse como medios porosos total o parcialmente saturados.

$\mathrm{Al}$ abordar el problema de la propagación de ondas sísmicas en medios porosos saturados, surge la necesidad de definir, de manera rigurosa, el medio poroso en sí. Es decir, debemos encontrar una serie de parámetros y variables que permitan definir su estado y evolución. A nivel microscópico, el medio poroso suele presentar geometrías significativamente complejas, por lo cual, una descripción matemática rigurosa resulta virtualmente impracticable (Bear, 1972). En este sentido, la descripción de un medio poroso suele abordarse empleando una formulación de medio continuo, a escala 
macroscópica.

Al utilizar una formulación de medio continuo se asume la existencia de variables de macroescala, definidas en volúmenes elementales representativos (REV). Es decir, se asume que para una determinada escala macroscópica es posible definir una serie de variables, dadas por promedios volumétricos de las propiedades de microescala, que permiten definir el estado del medio. Por ello, los REVs deben poseer el tamaño más pequeño posible que satisfaga que todas las variables macroscópicas empleadas, tales como, la porosidad o la permeabilidad, sean representativas del medio original. Biot (1962) empleó una formulación de medio continuo para desarrollar la teoría de la poroelasticidad. Como se verá en este Capítulo, la teoría de Biot se encuentra basada en una postulación Lagrangiana y hace uso del principio de Hamilton para derivar las ecuaciones de propagación de ondas en medios porosos.

A continuación abordaremos los fundamentos de la teoría de la poroelasticidad. Esta teoría considera, bajo ciertas hipótesis debidamente fundamentadas, que al estudiar la propagación de ondas sísmicas en medios porosos saturados por una única fase fluida, la matriz es isótropa y elástica. En particular, un aspecto notable de esta teoría es que los fluidos son libres de moverse dentro de la matriz. Esta característica posee ciertas consecuencias fenomenológicas, entre las cuales resalta la existencia de un modo compresional adicional a los modos de onda clásicos compresional (P) y de corte (S) presentes en sólidos elásticos y viscoelásticos. Esta onda adicional, comúnmente conocida como $\mathrm{P}_{2} \mathrm{u}$ onda lenta de Biot, se caracteriza por poseer una velocidad de fase menor a la de la onda $\mathrm{P}$ clásica. A su vez, la onda lenta se asocia con un movimiento fuera de fase de la matriz respecto del fluido poral. De forma singular, en el régimen de bajas frecuencias la onda lenta no se expresa como un modo de propagación, sino que toma la forma de un fenómeno de difusión, es decir, se comporta como un proceso que tiende a equilibrar los gradientes de presión poral presentes en el medio.

Luego, los modos de propagación de la teoría de Biot en bajas frecuencias pueden asociarse, de manera conceptual, a dos modos sísmicos ( $\mathrm{S}$ y $\mathrm{P}$ rápida) y un modo «hidráulico» $\left(\mathrm{P}_{2}\right)$. En particular, en presencia de heterogeneidades del medio poroso, los modos clásicos generan ondas $\mathrm{P}_{2}$ por conversión tipo «scattering》 (Pride, 2005). Por lo tanto, el estudio de la propagación de ondas sísmicas en medios porosos en el contexto de la teoría de Biot permite explorar las posibles conexiones entre los observables sísmicos y las propiedades hidráulicas del medio.

\subsection{Teoría de la poroelasticidad de Biot}

La teoría de Biot es ampliamente utilizada para analizar la propagación de ondas sísmicas en medios porosos saturados. A continuación, presentaremos una breve revisión de sus fundamentos.

\subsubsection{Hipótesis}

Como cualquier teoría física, los trabajos de Biot (1941, 1956a,b, 1962) están basados en ciertas hipótesis. Estas pueden resumirse de la siguiente manera (p. ej., Carcione, 2007; Bourbié et al., 1987): 
- El medio poroso está compuesto por una matriz sólida porosa continua, elástica, e isótropa.

- Los desplazamientos de la fases sólida y fluida son muy pequeños. Consecuentemente, las formulaciones Eulerianas y Lagrangianas coinciden hasta el primer orden. Esta hipótesis está justificada, ya que las deformaciones ejercidas por el paso de una onda sísmica son típicamente menores a $10^{-6}$ unidades.

- Es posible aplicar los principios de la mecánica de medios continuos para definir variables macroscópicas. Estas variables macroscópicas, dentro de la teoría de Biot, son promedios volumétricos de las variables microscópicas correspondientes.

- La longitud de onda es mucho mayor que las dimensiones de los volumen elementales empleados en la formulación de medio continuo para definir las variables macroscópicas. A su vez, estos volúmenes poseen propiedades, tales como, la porosidad, la permeabilidad, y los módulos elásticos, que son representativos del medio.

- Las condiciones termodinámicas son adiabáticas. Esta hipótesis es razonable, ya que la perturbación generada por el paso de una onda sísmica es un fenómeno que tiene lugar en un intervalo de tiempo significativamente breve.

- La fase fluida es continua y la distribución de tensiones dentro del fluido puede ser considerada hidrostática.

- No existen acoplamientos, en particular, acoplamientos termomecánicos.

Basándonos en estas hipótesis procederemos a introducir las variables características de la teoría de Biot. Luego, derivaremos las relaciones constitutivas correspondientes.

\subsubsection{Variables de la teoría de Biot}

Consideremos un volumen elemental $\Omega$ de material poroso saturado, centrado en la posición $\mathbf{x}$, que posee un tamaño mucho mayor al tamaño característico de los poros y granos sólidos, pero mucho más pequeño que las longitudes de onda sísmica predominantes. La porosidad en $\mathbf{x}$ se define como la relación entre el volumen poral $V_{p}$ y el volumen $V$ de $\Omega$, es decir,

$$
\phi(\mathbf{x})=\frac{V_{p}}{V}
$$

Sean $\mathbf{u}(\mathbf{x}, t)$ el desplazamiento promedio de las partículas de la matriz sólida de $\Omega$ para un tiempo $t$ y $\mathbf{U}(\mathbf{x}, t)$ el desplazamiento promedio de la fase fluida en $\Omega$ generados por el paso de una onda sísmica. Tanto $\mathbf{u}(\mathbf{x}, t)$ como $\mathbf{U}(\mathbf{x}, t)$ deben entenderse como un desplazamiento equivalente asignado al punto $\mathbf{x}$. Es de utilidad definir el vector de desplazamiento relativo del fluido,

$$
\mathbf{w}=\phi(\mathbf{U}-\mathbf{u})
$$

que representa el desplazamiento de fluido relativo al sólido. Es interesante notar que, para cualquier volumen macroscópico de material poroso $\Omega$ con frontera $\Gamma$, utilizando 
el teorema de la divergencia tenemos que

$$
\int_{\Gamma} \mathbf{w} \cdot \boldsymbol{\nu} \mathrm{d} S=\int_{\Omega} \nabla \cdot \mathbf{w} \mathrm{d} V
$$

donde $\boldsymbol{\nu}$ es el versor normal unitario exterior a $\Gamma$ y $\mathrm{d} S$ un diferencial de superficie. En particular, si el volumen $V$ es suficientemente pequeño como para considerar $\nabla \cdot \mathbf{w}$ constante en $\Omega$, la ecuación (2.3) resulta en

$$
\frac{1}{V} \int_{\Gamma} \mathbf{w} \cdot \boldsymbol{\nu} \mathrm{d} S \simeq \nabla \cdot \mathbf{w}
$$

Esta ecuación permite observar que $\nabla \cdot \mathbf{w}$ es una medida del cambio de contenido de fluido por unidad de volumen. Por ello, se define al cambio local en el contenido de fluido poral $\zeta$ como

$$
\zeta=-\nabla \cdot \mathbf{w}
$$

Si $\zeta$ es positivo en $\mathbf{x}$ el contenido de fluido aumenta. De manera contraria, si $\zeta$ es negativo el contenido de fluido en $\mathbf{x}$ disminuye.

Como la teoría de Biot es una teoría lineal, el tensor de deformaciones de la matriz sólida $\epsilon$ está dado por

$$
\boldsymbol{\epsilon}=\frac{1}{2}\left(\nabla \mathbf{u}+(\nabla \mathbf{u})^{\mathrm{T}}\right)
$$

donde $\mathrm{T}$ denota la transpuesta ${ }^{1}$.

En particular, la teoría de Biot hace uso del tensor de tensiones totales $\boldsymbol{\sigma}$. Este tiene en cuenta las tensiones actuantes sobre las fases sólida y fluida, y responde a (Cheng, 2016)

$$
\boldsymbol{\sigma}=(1-\phi) \boldsymbol{\sigma}^{s}-\phi p_{f} \mathbf{I}
$$

donde $p_{f}$ denota el cambio de presión del fluido poral debido a la perturbación generada por el paso de una onda sísmica, I es la matriz identidad, y $\boldsymbol{\sigma}^{s}$ el tensor de tensiones de la matriz sólida.

Una vez definidas las variables de la teoría de Biot, podemos abocarnos a analizar la expresión de la energía elástica de deformación del medio poroso saturado. Esta permitirá relacionar el tensor de tensiones $\boldsymbol{\sigma}$, el tensor de deformaciones $\boldsymbol{\epsilon}$, la presión del fluido poral $p_{f}$ y el cambio en el contenido de fluido $\zeta$.

\subsubsection{Densidad de energía elástica de deformación y relacio- nes constitutivas}

Consideremos que la perturbación generada por el paso de una onda sísmica es un fenómeno adiabático, ya que tiene lugar en un intervalo de tiempo significativamente breve. Luego, es razonable asumir la existencia de un potencial volumétrico interno $\mathcal{W}$, cuya variación representa el trabajo por unidad de volumen necesario para deformar la roca en una transformación infinitesimal macroscópica (p. ej., Bourbié et al., 1987). El potencial volumétrico $\mathcal{W}$, definido de esta manera, no es otra cosa que la densidad de

\footnotetext{
${ }^{1}$ El gradiente del vector desplazamiento es un tensor de segundo rango: $\nabla \mathbf{u}=\partial u_{j} / \partial x_{i} \check{\mathbf{e}}_{j} \otimes \check{\mathbf{e}}_{i}$.
} 
energía elástica de deformación del medio y, por lo tanto, depende de las componentes del tensor de deformaciones $\boldsymbol{\epsilon}$ y del cambio en el contenido de fluido $\zeta$, es decir,

$$
\mathcal{W}=\mathcal{W}(\epsilon, \zeta)
$$

Además, se considera que su variación d $\mathcal{W}$ responde a una forma diferencial exacta, es decir,

$$
\mathrm{d} \mathcal{W}=\frac{\partial \mathcal{W}}{\partial \epsilon_{i j}} \mathrm{~d} \epsilon_{i j}+\frac{\partial \mathcal{W}}{\partial \zeta} \mathrm{d} \zeta
$$

donde seguimos la notación de Einstein para la suma de subíndices repetidos. El primer miembro de la ecuación (2.9) corresponde al trabajo generado por deformación macroscópica a contenido de fluido constante, y el segundo término se asocia al trabajo generado por un incremento de fluido a deformación macroscópica fija. Luego, empleando el principio de los trabajos virtuales, se encuentra que el tensor de tensiones y la presión de fluido responden a

$$
\begin{aligned}
\sigma_{i j} & =\frac{\partial \mathcal{W}}{\partial \epsilon_{i j}}, \\
p_{f} & =\frac{\partial \mathcal{W}}{\partial \zeta} .
\end{aligned}
$$

Procedamos entonces a encontrar una expresión para $\mathcal{W}$ en función de los desplazamientos de las fases sólida y fluida.

Es importante recordar que la densidad de energía elástica de deformación es una función isótropa (Biot, 1962). Es decir, $\mathcal{W}$ depende de la deformación propiamente dicha, y no así de las rotaciones que pueda sufrir el sistema. Por lo tanto, esta densidad de energía es una función de los invariantes del tensor de deformación $I_{1}, I_{2}, I_{3}$, y del cambio en el contenido del fluido $\zeta$

$$
\mathcal{W}=\mathcal{W}\left(I_{1}, I_{2}, I_{3}, \zeta\right)
$$

Como Biot considera una relación lineal entre esfuerzo y deformación, las energías deben ser formas cuadráticas. Luego, $\mathcal{W}$ depende solamente de $\zeta$, y de los invariantes lineal y cuadrático del tensor de deformaciones $\boldsymbol{\epsilon}$,

$$
\begin{aligned}
& I_{1}(\epsilon)=\epsilon_{x x}+\epsilon_{y y}+\epsilon_{z z}, \\
& I_{2}(\epsilon)=\epsilon_{x x} \epsilon_{y y}+\epsilon_{x x} \epsilon_{z z}+\epsilon_{z z} \epsilon_{y y}-\epsilon_{x y} \epsilon_{y x}-\epsilon_{x z} \epsilon_{z x}-\epsilon_{y z} \epsilon_{z y},
\end{aligned}
$$

respectivamente. Entonces, la densidad de energía elástica en el contexto de la teoría de la poroelasticidad puede expresarse a través de la siguiente combinación lineal (Biot, 1962)

$$
2 \mathcal{W}=\left(\lambda_{c}+2 \mu\right) I_{1}^{2}-4 \mu I_{2}-2 \alpha M I_{1} \zeta+M \zeta^{2},
$$

donde $\lambda_{c}, \alpha, \mu, \mathrm{y} M$ son constantes a determinar.

Luego, empleando la ecuación (2.15) en (2.10) y (2.11), obtenemos las relaciones constitutivas para un medio poroelástico isótropo saturado por un único fluido poral

$$
\begin{gathered}
\boldsymbol{\sigma}=2 \mu \boldsymbol{\epsilon}+\mathbf{I}\left(\lambda_{c} \nabla \cdot \mathbf{u}-\alpha M \zeta\right), \\
p_{f}=-\alpha M \nabla \cdot \mathbf{u}+M \zeta
\end{gathered}
$$


Podemos observar que las tensiones $\boldsymbol{\sigma}$ y la presión $p_{f}$ se relacionan con las deformaciones $\boldsymbol{\epsilon}$ y el incremento en el contenido de fluido $\zeta$, a través de cuatro constantes: $\lambda_{c}, \alpha$, $\mu$, y $M$. A su vez, empleando las relaciones constitutivas (2.16) y (2.17) en la ecuación (2.15), es fácil demostrar que la energía de deformación responde a (Biot, 1941, 1956a)

$$
\mathcal{W}=\frac{1}{2}\left(\boldsymbol{\sigma}: \boldsymbol{\epsilon}+p_{f} \zeta\right)
$$

donde $\boldsymbol{\sigma}: \boldsymbol{\epsilon}=\sum_{i} \sum_{j} \sigma_{i j} \epsilon_{i j}$ denota la doble contracción de los tensores correspondientes.

Para conocer el significado de las constantes $\lambda_{c}, \alpha, \mu$ y $M$, se requiere de una serie de experimentos teóricos, denominados experimentos «gedanken» (p. ej., Biot y Willis, 1957). En breve, los resultados de esta serie de experimentos teóricos indican que el coeficiente $\mu$ es el módulo de corte clásico del medio poroso saturado, que es igual al módulo de corte de la matriz seca $\mu_{m}$ (Gassmann, 1951). El coeficiente $\lambda_{c}$ es comúnmente denominado constante de Lamé en estado «undrained», es decir, en un escenario donde no hay intercambio de fluido con el entorno $(\zeta=0$ en (2.16)). Por otro lado, el coeficiente $M$ es denominado coeficiente de Biot, y denota el incremento de la presión del fluido en respuesta a un aumento del contenido de fluido de una unidad, a deformación constante $(\nabla \cdot \mathbf{u}=0$ en $(2.17))$. Por último, el coeficiente $\alpha$, denominado comúnmente coeficiente de Biot-Willis, cuantifica la relación entre la variación en el contenido de fluido y la deformación macroscópica para un sistema abierto, donde el fluido puede escapar libremente $\left(p_{f}=0\right.$ en (2.17)). Las constantes poroelásticas pueden vincularse con las propiedades físicas del medio mediante las siguientes relaciones (p. ej., Masson y Pride, 2007)

$$
\begin{aligned}
\alpha & =1-\frac{K_{m}}{K_{s}} \\
\lambda_{c} & =K_{m}+\alpha^{2} M-\frac{2}{3} \mu, \\
M & =\left(\frac{\phi}{K_{f}}+\frac{\alpha-\phi}{K_{s}}\right)^{-1},
\end{aligned}
$$

donde $K_{f}, K_{s}$ y $K_{m}$ son los módulos de volumen ${ }^{2}$ del fluido, del material que compone a la matriz (p. ej., granos minerales), y de la matriz seca, respectivamente.

La teoría de Biot considera la existencia de una función de disipación, cuya definición es clave en la propagación de ondas en medios poroelásticos, y será analizada a continuación.

\subsubsection{Función de disipación}

Como ya se ha mencionado, cuando una onda se propaga por un medio poroelástico, el fluido poral puede experimentar desplazamientos respecto de la matriz. Este proceso puede generar disipación de la energía por fricción viscosa. La correspondiente fuerza disipativa $\mathbf{F}$ por unidad de volumen, depende de la velocidad relativa del fluido respecto de la matriz (Bourbié et al., 1987)

$$
F_{i}=-b \dot{\mathrm{w}}_{i}
$$

\footnotetext{
${ }^{2}$ Del inglés: «Bulk moduli».
} 
donde $b$ es un coeficiente de fricción y $\cdot$ denota la derivada temporal de la variable respectiva. Entonces, es posible definir una función de disipación $\mathcal{D}$ tal que $F_{i}=-\partial \mathcal{D} / \partial \dot{\mathrm{w}}_{i}$. En consecuencia, la función de disipación puede expresarse de acuerdo a (Biot, 1962)

$$
\mathcal{D}=\frac{1}{2} \dot{\mathbf{w}}^{\mathrm{T}} b \dot{\mathbf{w}} .
$$

La expresión del coeficiente de fricción $b$ en función de las propiedades físicas del medio se discutirá en conjunto con las ecuaciones de movimiento.

\subsubsection{Densidad de energía cinética}

La densidad de energía cinética macroscópica no puede obtenerse sumando la energías cinéticas del sólido y el fluido en términos de $\mathbf{u}$ y w, ya que estas no son las verdaderas velocidades (microscópicas), sino velocidades promedio. Es por ello que Biot postula una forma cuadrática para la densidad de energía cinética que contiene un término de acople, es decir (Bourbié et al., 1987)

$$
\mathcal{C}=\frac{1}{2} \rho_{b} \dot{\mathbf{u}}^{\mathrm{T}} \dot{\mathbf{u}}+\rho_{f} \dot{\mathbf{w}}^{\mathrm{T}} \dot{\mathbf{u}}+\frac{1}{2} g \dot{\mathbf{w}}^{\mathrm{T}} \dot{\mathbf{w}}
$$

donde $\rho_{f}$ denota la densidad del fluido poral, $\rho_{b}=(1-\phi) \rho_{s}+\phi \rho_{f}$ es la densidad promedio del agregado ${ }^{3}$, y $\rho_{s}$ denota la densidad de los granos sólidos. Además, se define el factor de acoplamiento de masa $g$, el cual cuantifica la interacción entre las fases sólida y fluida. Este tiene en cuenta que el movimiento del fluido no se da necesariamente en la dirección del gradiente de presión macroscópico, debido a la tortuosidad del espacio poral, y responde a

$$
g=\frac{\tau \rho_{f}}{\phi}
$$

donde $\tau$ denota la tortuosidad de la roca. La ausencia de términos del tipo $\dot{\mathrm{w}}_{i} \dot{\mathrm{w}}_{j}$ en la ecuación (2.24) se debe a que hemos asumido que el material es isótropo.

A continuación, empleando $\mathcal{W}, \mathcal{D}$, y $\mathcal{C}$ derivaremos las ecuaciones de movimiento de un medio poroso saturado.

\subsubsection{Ecuaciones de movimiento}

Luego de introducir las expresiones de la energía elástica de deformación $\mathcal{W}$, la función de disipación $\mathcal{D}$, y la energía cinética $\mathcal{C}$, procederemos a derivar las ecuaciones de movimiento en un medio poroelástico. En este sentido, la teoría de Biot (1956a,b) postula la validez de los conceptos de la mecánica del continuo y en particular de las ecuaciones de Lagrange a escala macroscópica.

Por un lado, el Lagrangiano por unidad de volumen $\mathcal{L}$, definido en base a la densidad de energía cinética (2.24) y al potencial de deformación (2.18), responde a

$$
\mathcal{L}=\mathcal{C}-\mathcal{W}
$$

Por el otro lado, el principio de Hamilton establece que, entre todos los campos de desplazamiento posibles que satisfacen las condiciones de contorno de un problema, el

\footnotetext{
${ }^{3} \rho_{b}$ es denominada densidad «bulk» en los textos en inglés.
} 
que realmente ocurre es aquel que hace estacionaria la integral sobre tiempo y espacio de $\mathcal{L}$ y del trabajo de las fuerzas disipativas. Estas últimas se derivan de la función de disipación $\mathcal{D}$ (2.23). Luego, las ecuaciones de Euler Lagrange responden a (p. ej., Goldstein y Safko, 2014)

$$
\frac{\partial}{\partial t} \frac{\partial \mathcal{L}}{\partial \dot{\mathfrak{q}}_{i}}+\frac{\partial}{\partial x_{j}} \frac{\partial \mathcal{L}}{\partial \mathfrak{q}_{i, j}}-\frac{\partial \mathcal{L}}{\partial \mathfrak{q}_{i}}+\frac{\partial \mathcal{D}}{\partial \dot{\mathfrak{q}}_{i}}=0
$$

donde $\mathfrak{q}_{i}$, con $i=x, y, z$, son las coordenadas generalizadas del problema. Estas se encuentran asociadas a las componentes del desplazamiento promedio de la matriz sólida $\mathrm{u}_{i} \mathrm{y}$ las componentes del desplazamiento relativo del fluido $\mathrm{w}_{i}$. Además, denotamos $\mathfrak{q}_{i, j}=\partial \mathfrak{q}_{i} / \partial x_{j}$

Luego, empleando las expresiones de $\mathcal{L}$ y $\mathcal{D}$ en la ecuación (2.27), se obtienen las ecuaciones de movimiento en el dominio del espacio y el tiempo

$$
\begin{gathered}
\nabla \cdot \boldsymbol{\sigma}=\rho_{b} \ddot{\mathbf{u}}+\rho_{f} \ddot{\mathbf{w}}, \\
-\nabla p_{f}=\rho_{f} \ddot{\mathbf{u}}+g \ddot{\mathbf{w}}+b \dot{\mathbf{w}} .
\end{gathered}
$$

Las ecuaciones (2.28)-(2.29) acopladas mediante las relaciones constitutivas (2.16)(2.17), permiten estudiar la propagación de ondas sísmicas en medios porosos saturados por un fluido viscoso compresible. Es común que este juego de ecuaciones se exprese en el dominio del espacio y la frecuencia, es decir,

$$
\begin{gathered}
\nabla \cdot \hat{\boldsymbol{\sigma}}=-\omega^{2} \rho_{b} \hat{\mathbf{u}}-\omega^{2} \rho_{f} \hat{\mathbf{w}} \\
-\nabla \hat{p}_{f}=-\omega^{2} \rho_{f} \hat{\mathbf{u}}-\omega^{2} g \hat{\mathbf{w}}+i \omega b \hat{\mathbf{w}},
\end{gathered}
$$

donde $\omega$ es la frecuencia angular. Empleamos la notación ^ para expresar los parámetros asociados en el dominio de la frecuencia. Trabajar en el espacio y la frecuencia presenta ciertas ventajas algebraicas a la hora de operar numéricamente y, por ello, la mayor parte de los cómputos realizados en este trabajo de Tesis se llevan a cabo en este dominio.

Procedamos entonces a identificar el coeficiente de fricción $b$. En el caso estacionario $\left(\ddot{\mathrm{w}}_{i}=0\right.$ y $\left.\ddot{\mathrm{u}}_{i}=0\right)$, la ecuación $(2.29)$ se reduce a $-\nabla p_{f}=b \dot{\mathbf{w}}$. Entonces, por analogía con la ley de Darcy, se puede definir

$$
b=\frac{\eta}{\kappa},
$$

donde $\eta$ y $\kappa$ son la viscosidad dinámica del fluido y la permeabilidad de la matriz.

Es importante resaltar que las expresiones brindadas en este Capítulo para los parámetros $b$ y $g$, ecuaciones (2.25) y (2.32), son correctas siempre y cuando el flujo sea laminar, es decir, si las fuerzas viscosas predominan por sobre las fuerzas inerciales. En caso contrario, se comienzan a desarrollar capas límites viscosas dentro del espacio poral (Cheng, 2016). Para que el flujo sea considerado laminar, el espesor de la capa límite viscosa debe ser mayor al radio poral. Esta condición se sostiene para frecuencias menores a la frecuencia crítica de Biot (Johnson et al., 1987)

$$
\omega_{\text {Biot }}=\frac{\phi \eta}{\tau \rho_{f} \kappa} .
$$


Para frecuencias mayores a $\omega_{\text {Biot }}$, el flujo deja de ser laminar y es necesario considerar una dependencia de $b$ y de $g$ con la frecuencia (p. ej., Johnson et al., 1987). En general, el valor de $\omega_{\text {Biot }}$ es mucho mayor a las frecuencias usualmente empleadas en la sísmica de exploración, y las expresiones (2.25) y (2.32) son aceptables en la mayoría de las aplicaciones geofísicas.

A continuación introduciremos brevemente las soluciones de las ecuaciones de onda, probando, entre otras cosas, la existencia de la onda lenta de Biot.

\subsubsection{Soluciones de las ecuaciones de movimiento}

Consideremos el teorema de descomposición de Helmholtz para desacoplar los campos de onda compresionales y de corte (p. ej., Tod, 2003). Para ello, asumimos que los desplazamientos $\hat{\mathbf{u}} \mathrm{y} \hat{\mathbf{w}}$ poseen una componente irrotacional y otra isovolumétrica, es decir,

$$
\left\{\begin{array}{c}
\hat{\mathbf{u}} \\
\hat{\mathbf{w}}
\end{array}\right\}=\left\{\begin{array}{c}
\nabla \Phi_{1} \\
\nabla \Phi_{2}
\end{array}\right\}+\left\{\begin{array}{c}
\nabla \times \boldsymbol{\Psi}_{1} \\
\nabla \times \boldsymbol{\Psi}_{2}
\end{array}\right\},
$$

donde $\Phi_{1}$ y $\Phi_{2}$ son potenciales escalares, y $\boldsymbol{\Psi}_{1}$ y $\boldsymbol{\Psi}_{2}$ potenciales vectoriales. De esta manera, podemos encontrar soluciones independientes para las ecuaciones de onda compresional y de corte.

\section{Ondas compresionales}

En efecto, al considerar la propagación de una onda plana compresional monocromática, de frecuencia $\omega$, a través de un medio poroelástico, el desplazamiento del sólido y del fluido pueden expresarse de acuerdo a

$$
\begin{aligned}
& \hat{\mathbf{u}}(\mathbf{x}, \omega)=\nabla \Phi_{1}, \\
& \hat{\mathbf{w}}(\mathbf{x}, \omega)=\nabla \Phi_{2} .
\end{aligned}
$$

Consideramos que

$$
\begin{aligned}
& \Phi_{1}=A_{c} e^{i\left(\omega t-\mathbf{k}_{\mathbf{c}} \cdot \mathbf{x}\right)}, \\
& \Phi_{2}=B_{c} e^{i\left(\omega t-\mathbf{k}_{\mathbf{c}} \cdot \mathbf{x}\right)},
\end{aligned}
$$

donde $\mathbf{k}_{\mathbf{c}}$ denota el vector de onda compresional. Para ondas homogéneas, $\mathbf{k}_{\mathbf{c}}$ puede escribirse de acuerdo a

$$
\mathbf{k}_{\mathbf{c}}=k_{c}(\omega) \check{\mathbf{k}}_{\mathbf{c}}
$$

donde $k_{c}(\omega)$ es el número de onda complejo y $\check{\mathbf{k}}_{\mathbf{c}}$ es un vector unitario en la dirección de propagación de la onda. Como consideramos ondas planas compresionales, $\hat{\mathbf{u}}, \hat{\mathbf{w}} \mathrm{y}$ $\check{\mathbf{k}}_{\mathbf{c}}$ son paralelos. Por lo cual, sin pérdida de generalidad, podemos tomar el caso de una onda compresional monocromática propagándose en la dirección del eje $x$, de manera que $\check{\mathbf{k}}_{\mathbf{c}}=\check{\mathbf{e}}_{\mathbf{1}}$, con la intención de simplificar los cálculos.

Procedemos entonces a introducir el juego de ecuaciones (2.35) y (2.36) en las ecuaciones de movimiento (2.30)-(2.31) y, empleando las relaciones constitutivas (2.16)(2.17), obtenemos el siguiente sistema de ecuaciones

$$
\begin{array}{r}
{\left[-\rho_{b} \omega^{2}+H k_{c}(\omega)^{2}\right] A_{c}+\left[-\omega^{2} \rho_{f}+\alpha M k_{c}(\omega)^{2}\right] B_{c}=0} \\
{\left[-\rho_{f} \omega^{2}+\alpha M k_{c}(\omega)^{2}\right] A_{c}+\left[-\omega^{2} g+i b \omega+M k_{c}(\omega)^{2}\right] B_{c}=0}
\end{array}
$$


donde $H=\lambda_{c}+2 \mu$. Para que este sistema lineal de ecuaciones posea solución, más allá de la solución trivial $B_{c}=A_{c}=0$, su determinante debe ser nulo. Esta condición da lugar a una ecuación bicuadrática en $k_{c}(\omega)$, la cual posee cuatro soluciones matemáticamente válidas. Sin embargo, solamente dos de ellas son físicamente aceptables. Para ello, las soluciones deben cumplir que la parte imaginaria del número de onda complejo sea negativa $\Im\left\{k_{c}\right\}<0$. Esta condición, como puede observarse de las expresiones de los desplazamientos

$$
\begin{aligned}
& \hat{\mathbf{u}}=-i k_{c} A_{c} e^{\Im\left\{k_{c}\right\} x} e^{i\left(\omega t-\Re\left\{k_{c}\right\} x\right)} \check{\mathbf{e}}_{\mathbf{1}}, \\
& \hat{\mathbf{w}}=-i k_{c} B_{c} e^{\Im\left\{k_{c}\right\} x} e^{i\left(\omega t-\Re\left\{k_{c}\right\} x\right)} \check{\mathbf{e}}_{\mathbf{1}},
\end{aligned}
$$

asegura que las ondas no aumentan su amplitud al propagarse, sino que, de forma contraria, su amplitud disminuye. Luego, existen dos posibles ondas compresionales, usualmente denominadas onda rápida $\mathrm{P}$ y onda lenta $\mathrm{P}_{2}$, con números de onda compresionales $k_{\mathrm{P}}$ y $k_{\mathrm{P}_{2}}$.

Para frecuencias mucho menores que la frecuencia crítica de Biot $\left(\omega<<\omega_{\text {Biot }}\right)$, las fuerzas viscosas dominan por sobre las fuerzas inerciales y, entonces, las fases sólida y fluida tienden a moverse de forma conjunta frente al paso de una onda $\mathrm{P}$ rápida. Como el desplazamiento relativo del fluido es prácticamente nulo, la atenuación y la dispersión son despreciables. Para frecuencias mucho mayores que la frecuencia crítica de Biot $\left(\omega>>\omega_{\text {Biot }}\right)$, los efectos inerciales del fluido son significativos. Sin embargo, la capa límite es muy delgada y las fuerzas viscosas no logran hacer trabajo. Por esta razón, la atenuación y la dispersión de la onda $\mathrm{P}$ son despreciables también en este rango de frecuencias. Al considerar frecuencias cercanas a $\omega_{\text {Biot }}$, el espesor de la capa límite es del orden del radio poral, de modo que prácticamente todo el fluido está afectado por las fuerzas viscosas y, además, el desplazamiento relativo del fluido es considerable. En este caso, puede observarse una significativa atenuación y dispersión sísmica. Es interesante hacer notar que, un segundo mecanismo de disipación puede tener lugar en la propagación de una onda $\mathrm{P}$ rápida (Pride, 2005). Este ocurre cuando (y si) existe tiempo suficiente en un período de onda para que se equilibren las presiones entre las regiones del medio sujetas a compresión y aquellas sujetas a extensión. Sin embargo, puede demostrarse que este mecanismo de relajación no opera en la mayor parte de las rocas sedimentarias (Pride, 2005). En conjunto, estos mecanismos de disipación conforman el comúnmente denominado mecanismo intrínseco de Biot. Este tiene lugar, primordialmente, en el rango de frecuencias ultrasónico (p. ej. Müller et al., 2010; Johnston et al., 1979; Winkler, 1985; Pride, 2005).

Por otro lado, la onda lenta $\mathrm{P}_{2}$ está caracterizada por un movimiento fuera de fase del fluido respecto de la matriz. Para altas frecuencias $\left(\omega>>\omega_{\text {Biot }}\right)$, la onda $P_{2}$ es un modo de propagación. Sin embargo, en el régimen de bajas frecuencias $\left(\omega<<\omega_{\text {Biot }}\right)$, la onda $\mathrm{P}_{2}$ es altamente dispersiva $\mathrm{y}$, de hecho, toma la forma de un proceso de difusión de la presión del fluido (Dutta y Odé, 1979a). La velocidad de la onda lenta de Biot está asociada a la velocidad propagación de los cambios en la presión poral a través del medio poroso (Mavko et al., 2009). Es importante resaltar que, en medios heterogéneos, las ondas $\mathrm{P}$ y $\mathrm{S}$ pueden generar ondas $\mathrm{P}_{2}$ por conversión de energía en las interfases de las heterogeneidades. Las implicancias de este proceso serán analizadas con mayor profundidad en la Sección 2.3. Como hemos comentado previamente, la confirmación experimental de la existencia de la onda lenta $\mathrm{P}_{2}$ fue llevada a cabo, por primera vez, 
por Plona y Johnson (1980).

\section{Ondas de corte}

$\mathrm{Al}$ analizar los posibles desplazamientos transversales asociados a ondas de corte, los desplazamientos del sólido y del fluido pueden expresarse de acuerdo a

$$
\begin{aligned}
\hat{\mathbf{u}}(\mathbf{x}, \omega) & =\nabla \times \boldsymbol{\Psi}_{1}, \\
\hat{\mathbf{w}}(\mathbf{x}, \omega) & =\nabla \times \boldsymbol{\Psi}_{2} .
\end{aligned}
$$

Las expresiones de los potenciales vectoriales $\boldsymbol{\Psi}_{1}$ y $\boldsymbol{\Psi}_{2}$ responden a

$$
\begin{aligned}
& \boldsymbol{\Psi}_{1}=\mathbf{A}_{\mathbf{s}} e^{i\left(\omega t-\mathbf{k}_{\mathbf{s}} \cdot \mathbf{x}\right)}, \\
& \boldsymbol{\Psi}_{2}=\mathbf{B}_{\mathbf{s}} e^{i\left(\omega t-\mathbf{k}_{\mathbf{s}} \cdot \mathbf{x}\right)},
\end{aligned}
$$

donde $\mathbf{A}_{\mathbf{s}}$ y $\mathbf{B}_{\mathbf{s}}$ son los vectores que determinan la dirección, sentido, y amplitud de los potenciales, $\mathbf{y} \mathbf{k}_{\mathbf{s}}$ es el vector número de onda. En el caso de una onda plana monocromática S propagándose en un medio homogéneo e isótropo, los desplazamientos $\hat{\mathbf{u}} \mathrm{y} \hat{\mathbf{w}}$ son paralelos, pero ortogonales a $\mathbf{k}_{\mathbf{s}}$. De modo que, sin pérdida de generalidad podemos considerar el caso de una onda plana que se propaga en la dirección del eje $x$ y que tanto $\boldsymbol{\Psi}_{1}$ como $\boldsymbol{\Psi}_{2}$ están contenidas en el eje $z$. En este contexto, al introducir el juego de ecuaciones (2.41) y (2.42) en las ecuaciones de movimiento (2.30)-(2.31), considerando las relaciones constitutivas (2.16)-(2.17), obtenemos

$$
\begin{array}{r}
{\left[-\rho_{b}+\mu\left(\frac{k_{s}(\omega)}{\omega}\right)^{2}\right] A_{s}-\rho_{f} B_{s}=0} \\
-\rho_{f} A_{s}+\left[-g+\frac{i b}{\omega}\right] B_{s}=0
\end{array}
$$

donde $A_{s}, B_{s}$, y $k_{s}(\omega)$ son los módulos de los vectores $\mathbf{A}_{\mathbf{s}}, \mathbf{B}_{\mathbf{s}}, \mathrm{y} \mathbf{k}_{\mathbf{s}}$, respectivamente. Nuevamente, si deseamos que el sistema tenga solución más allá de la solución trivial $B_{s}=A_{s}=0$, el determinante del sistema debe ser nulo. Al plantear esta condición sobre (2.43), obtenemos que el modulo complejo para una onda $\mathrm{S}$ responde a

$$
k_{s}(\omega)=\left[\frac{\omega^{2}}{\mu}\left(\rho_{b}-\frac{\rho_{f}^{2}}{g-i \frac{b}{\omega}}\right)\right]^{\frac{1}{2}} .
$$

En este caso, existen dos posibles soluciones para $k_{s}(\omega)$. Elegimos aquella raíz que posee una parte imaginaria negativa. Para justificar esta elección, podemos observar que los desplazamientos

$$
\begin{aligned}
& \hat{\mathbf{u}}=i k_{s} A_{s} e^{\Im\left\{k_{s}\right\} x} e^{i\left(\omega t-\Re\left\{k_{s}\right\} x\right)} \check{\mathbf{e}}_{\mathbf{2}}, \\
& \hat{\mathbf{w}}=i k_{s} B_{s} e^{\Im\left\{k_{s}\right\} x} e^{i\left(\omega t-\Re\left\{k_{s}\right\} x\right)} \check{\mathbf{e}}_{\mathbf{2}},
\end{aligned}
$$

disminuyen su amplitud al propagarse la onda a través del medio si $\Im\left\{k_{s}\right\}<0$. Por lo cual, en el caso de ondas de corte, existe un único modo de propagación. Este posee, tal como la onda $\mathrm{P}$ rápida, una pérdida máxima de energía por fricción viscosa para 
frecuencias cercanas a $\omega_{\text {Biot }}$, mientras que es despreciable en el límite de bajas y de altas frecuencias. En el caso de la onda S, la atenuación y dispersión del medio se debe solo a efectos de aceleración de la matriz.

Como hemos mencionado, las ecuaciones de Biot poseen ciertas características particulares en el régimen de bajas frecuencias. A continuación, exploraremos las ecuaciones de consolidación de Biot.

\subsubsection{Ecuaciones de consolidación de Biot}

La ecuaciones de consolidación o ecuaciones cuasiestáticas de la teoría de la poroelasticidad (Biot, 1941) consisten en una aproximación de bajas frecuencias de las ecuaciones de movimiento (2.30)-(2.31). Esta aproximación permite estudiar de forma directa el mecanismo de difusión por relajación de presión poral asociado a la onda lenta de Biot, para un fluido monofásico. Las ecuaciones cuasiestáticas de Biot, en el dominio del espacio y la frecuencia, responden a (p. ej., Biot, 1941)

$$
\begin{gathered}
\nabla \cdot \hat{\sigma}=0, \\
-\nabla \hat{p}_{f}=\frac{\eta}{\kappa} i \omega \hat{\mathbf{w}} .
\end{gathered}
$$

La ecuación (2.46) representa el equilibrio de tensiones dentro del medio, mientras que la ecuación (2.47) es la ley de Darcy. Utilizando las relaciones constitutivas (2.16)-(2.17) y las ecuaciones de consolidación (2.46)-(2.47), se deriva directamente la ecuación de difusión (p. ej., Carcione, 2007)

$$
D \nabla^{2} \hat{p}_{f}=i \omega \hat{p}_{f}
$$

donde $D$ es la difusividad del medio y responde a

$$
D=\frac{\kappa}{\eta}\left(\frac{M H-\alpha^{2} M^{2}}{H}\right) .
$$

La ecuación (2.48) evidencia que el movimiento relativo del fluido asociado a la onda $\mathrm{P}_{2}$ en bajas frecuencias, responde a un proceso de difusión de presión. Se suele definir la longitud característica del proceso difusivo para procesos oscilatorios en el dominio del espacio y la frecuencia, denominada longitud de difusión, de acuerdo a (Norris, 1993)

$$
L_{d}=\sqrt{\frac{D}{\omega}} .
$$

La longitud de difusión cuantifica la escala espacial para la cual el proceso difusivo es significativo.

Las ecuaciones de consolidación de Biot (2.46) a (2.47) suelen ser empleadas para explorar los efectos del proceso de flujo inducido por onda en la mesoescala. A continuación abordaremos las particularidades de este proceso de disipación. 


\subsection{Flujo inducido por onda en la mesoescala}

Se considera que el mecanismo de flujo inducido por onda en la mesoescala puede contribuir de forma significativa a los altos niveles de disipación típicamente observados en la sísmica de exploración (Pride et al., 2004; Pride, 2005). Como hemos comentado previamente, este mecanismo de disipación tiene lugar cuando el medio poroso posee heterogeneidades con compresibilidades contrastantes, tales como, distribuciones irregulares de los fluidos porales y/o cambios en las propiedades de la matriz. En particular, estas heterogeneidades deben ser de escalas mucho mayores al tamaño característico de los poros, pero mucho menores que las longitudes de onda predominantes. Luego, las diversas regiones del medio poroso heterogéneo responden de manera diferente a la deformación causada por el paso de una onda sísmica, generando así gradientes de presión en los fluidos saturantes. Estos gradientes de presión tenderán a relajarse por medio de flujo a escalas mesoscópicas, disipando así la energía de la onda por fricción viscosa.

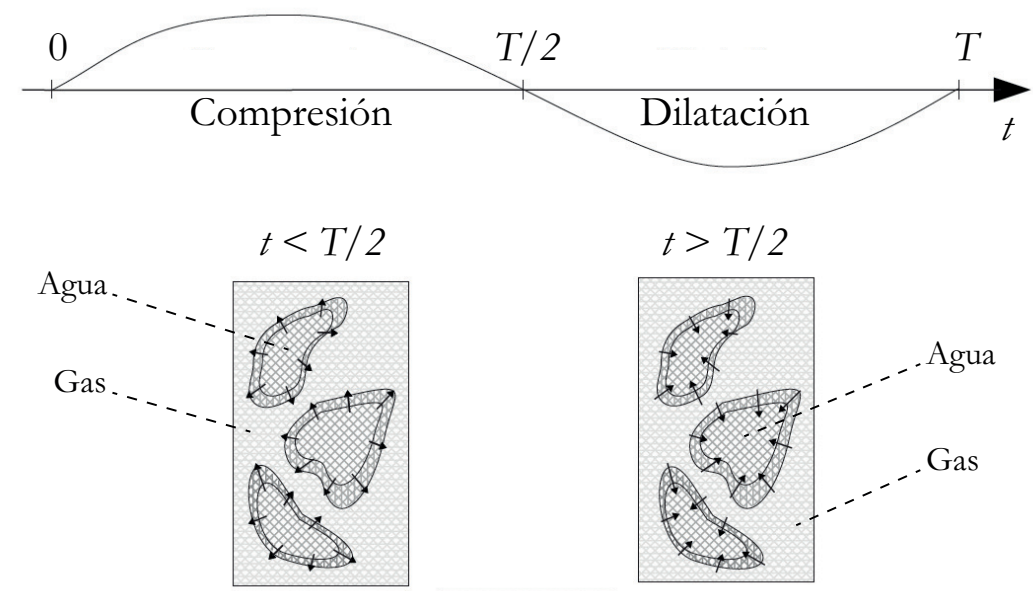

Figura 2.1. Ilustración esquemática del proceso de flujo inducido en respuesta al campo de tensión generado por una onda compresional P de período T. Durante el ciclo compresivo los «patches» saturados por el fluido con mayor módulo de volumen (agua) inyectarán fluido hacia el entorno saturado por un fluido con menor módulo de volumen (gas). En esta figura la dirección del flujo está indicada con flechas. Durante el ciclo de extensión, la dirección del flujo es inversa (modificado de Müller et al. (2010)).

La Figura 2.1 esquematiza el proceso de flujo inducido por el paso de una onda compresional P. En este caso, la matriz es homogénea y se encuentra saturada por dos fluidos inmiscibles de diferentes compresibilidades. Consideremos que las regiones aisladas de la Figura 2.1 están saturadas por un fluido poco compresible (alto módulo de volumen) como el agua y que las zonas restantes están saturadas con un fluido muy compresible (bajo módulo de volumen) como el gas. Las regiones saturadas por agua responden a la compresión generada por la onda con un mayor incremento de presión del fluido poral en comparación con el cambio de presión experimentado en las regiones saturadas por gas. Debido a esta diferencia de presiones, las regiones saturadas por 
agua inyectan fluido en su entorno inmediato, saturado por gas. Durante los períodos extensionales de la onda sísmica, la dirección del flujo es inversa. Este movimiento oscilatorio genera disipación de la energía sísmica por fricción viscosa y, por ello, la onda compresional puede verse significativamente atenuada.

Como hemos observado en la ecuación (2.50), la longitud característica del proceso difusivo, dada por $L_{d}$, depende de la frecuencia. Consecuentemente, pueden definirse diferentes regímenes para el proceso de flujo inducido por onda en la mesoescala dependiendo de la frecuencia de la onda sísmica. Cuando las frecuencias son muy bajas, la longitud de difusión es mucho mayor que el tamaño de las heterogeneidades y, por lo tanto, habrá suficiente tiempo en cada semiciclo de oscilación para que la presión del fluido poral del medio heterogéneo se equilibre a un valor común. Por lo tanto, el régimen de bajas frecuencias representa un estado relajado. En el otro extremo, para frecuencias suficientemente altas, en un semiciclo de oscilación no hay tiempo suficiente para que se equilibren las presiones porales entre las diferentes partes del medio $\mathrm{y}$, por lo tanto, el régimen de altas frecuencias se encuentra asociado a un estado no relajado. Sin embargo, para una determinada frecuencia intermedia, que denominaremos frecuencia crítica $\omega_{c}$, las longitudes de difusión son comparables con la longitud característica de las heterogeneidades $a_{\text {meso }}$, es decir,

$$
L_{d}\left(\omega_{c}\right) \propto a_{\text {meso }}
$$

En este escenario, el flujo asociado al paso de una onda sísmica se torna significativo. Por lo tanto, la frecuencia característica

$$
\omega_{c}=\frac{D}{L_{d}^{2}} \propto \frac{D}{a_{\mathrm{meso}}^{2}},
$$

está asociada con la ubicación del máximo de atenuación de las ondas sísmicas por flujo inducido. Es decir, el máximo de atenuación está determinado, en parte, por la longitud característica de las heterogeneidades $a_{\text {meso }}$. Por lo cual, se deduce que el dato sísmico puede contener información sobre las características geométricas de los patrones de distribución de fluidos que se encuentran por debajo de su resolución. Por otro lado, la difusividad $D$ (ecuación (2.49)) es directamente proporcional a la permeabilidad del medio $\kappa$. Luego, considerando (2.52), la frecuencia característica del proceso difusivo $\omega_{c}$ es directamente proporcional a la permeabilidad de la región donde tiene lugar la disipación. Por esta razón, los métodos sísmicos poseen la potencialidad de proveer información de gran valor respecto de los fluidos alojados en el subsuelo y de las propiedades hidráulicas de los reservorios.

Es importante resaltar que el flujo mesoscópico también puede ser inducido por el paso de una onda de corte $\mathrm{S}$, si el medio poroso contiene heterogeneidades de mesoescala en la matriz. La condición requerida para que una onda $\mathrm{S}$ genere gradientes locales de presión es que estas heterogeneidades posean una geometría anisótropa (Masson y Pride, 2007). Ejemplos de esta geometría anisótropa pueden ser una arenisca que posee lentes de sedimentos «shale» o fracturas. Las geometrías isótropas, tales como, inclusiones esféricas, no permiten que los esfuerzos de corte generados por el paso de un campo de ondas $\mathrm{S}$ generen cambios en la presión del fluido poral. 


\subsection{Conclusiones}

En este Capítulo hemos presentado las bases de la teoría de la poroelasticidad de Biot, introduciendo las variables, las relaciones constitutivas, y la ecuación de movimiento para medios poroelásticos. Demostramos que la teoría de Biot considera la existencia de un modo de propagación adicional a los modos clásicos compresional $\mathrm{P}$ y de corte $\mathrm{S}$, denominado onda lenta compresional $\mathrm{P}_{2}$. Esta última puede asociarse, para frecuencias suficientemente bajas, a un proceso de difusión de presión. En este sentido, se introdujeron las ecuaciones de movimiento cuasiestáticas de Biot, las cuales permiten modelar el proceso de difusión de presión asociado a la onda lenta compresional. En este contexto, hemos definido el mecanismo de flujo inducido por onda en la mesoescala, el cual contempla la disipación de la energía sísmica debido a la presencia de heterogeneidades de escalas mayores a la dimensión poral y menores que las longitudes de onda predominantes. A pesar de no poder evidenciar de manera directa la presencia de estas heterogeneidades en el dato sísmico, ya que se encuentran por debajo de su resolución característica, su presencia genera cambios en las amplitudes y velocidades de las ondas sísmicas. Consecuentemente, las mediciones de atenuación y dispersión de la velocidad pueden proveer información valiosa sobre las características de los medios porosos saturados. En particular, poseen la capacidad de brindar información sobre la permeabilidad del medio y las características geométricas de las distribuciones de fluidos.

La teoría de Biot provee un marco adecuado para estudiar los efectos de distribuciones mesoscópicas de fluidos inmiscibles en las firmas sísmicas, por ello será utilizada en este trabajo de Tesis. En el próximo Capítulo presentaremos diferentes metodologías para estimar la atenuación sísmica debida a flujo inducido por onda en rocas porosas heterogéneas. 


\section{Capítulo 3}

\section{Modelado de atenuación sísmica en medios porosos heterogéneos}

Con el objetivo de explorar los efectos del flujo inducido a mesoescala sobre las ondas sísmicas, se han desarrollado diversos modelos numéricos y analíticos de distintas complejidades. Estos modelos asumen que la respuesta sísmica de una roca poroelástica heterogénea en la mesoescala puede ser representada por un sólido viscoelástico homogéneo equivalente. Es decir, estas metodologías se basan en la comúnmente llamada hipótesis de medio viscoelástico equivalente. Sin embargo, aseverar que un medio viscoelástico homogéneo efectivo puede representar la atenuación y la dispersión de las ondas sísmicas debidas a flujo inducido por onda en medios porosos heterogéneos no es algo trivial. En este Capítulo describiremos el procedimiento generalmente utilizado para estimar la atenuación sísmica debida a flujo inducido por onda y presentaremos una novedosa formulación, completamente basada en la teoría de la poroelasticidad y que no hace uso de la hipótesis de medio viscoelástico equivalente. Esta metodología alternativa emplea la potencia disipada y la energía elástica almacenada en respuesta a un experimento oscilatorio numérico de relajación. Emplearemos este procedimiento para calcular la atenuación sísmica en una serie de escenarios pertinentes, que incluyen rocas parcialmente saturadas y rocas fracturadas, evaluando así el alcance de la hipótesis de medio viscoelástico equivalente. Además, a diferencia de las metodologías clásicas, el procedimiento propuesto posee la ventaja de proveer las contribuciones locales a la atenuación del medio. Estas permiten explorar las características internas de la disipación viscosa en el medio estudiado. Los resultados que se presentan en este Capítulo forman parte de los trabajos de Solazzi et al. (2014) y Solazzi et al. (2016).

\subsection{Introducción}

La atenuación que presentan las ondas sísmicas al atravesar un medio poroso saturado contiene información valiosa sobre las propiedades mecánicas e hidráulicas del mismo. Usualmente, se emplea el inverso del factor de calidad $Q^{-1}$ como medida no dimensional de la atenuación en términos del cociente entre la potencia disipada y la energía elástica almacenada en el medio. La definición del factor de calidad $Q$ proviene de la teoría de circuitos eléctricos y ha sido adoptada por las Geociencias empleando diversas definiciones (Green, 1955).

La gran mayoría de los modelos desarrollados para cuantificar la atenuación sísmica en presencia de heterogeneidades de mesoescala se basan en la hipótesis de existencia de un medio viscoelástico equivalente (p. ej., Masson y Pride, 2007; Rubino et al., 
2009; Quintal et al., 2011). Esta hipótesis considera que una roca porosa heterogénea sujeta a un campo de tensiones o deformaciones puede ser representada por un sólido viscoelástico homogéneo que exhibe la misma respuesta promedio en función de la frecuencia. Esto permite calcular los módulos viscoelásticos complejos equivalentes de onda plana y de corte del medio estudiado, a través del comportamiento promedio de la roca sometida al campo de tensión o deformación. Luego, de acuerdo a la teoría de la viscoelasticidad clásica, el inverso del factor de calidad $1 / Q^{\mathcal{D}}$, que cuantifica la atenuación del medio, se obtiene mediante la relación entre la parte imaginaria y la parte real de módulo complejo correspondiente (p. ej., Borcherdt, 2009)

$$
\frac{1}{Q_{p}^{\mathcal{D}}(\omega)}=\frac{\Im\left\{M_{c}(\omega)\right\}}{\Re\left\{M_{c}(\omega)\right\}}, \quad \frac{1}{Q_{s}^{\mathcal{D}}(\omega)}=\frac{\Im\left\{\mu_{c}(\omega)\right\}}{\Re\left\{\mu_{c}(\omega)\right\}},
$$

donde $M_{c}(\omega)$ y $\mu_{c}(\omega)$ son los módulos complejos equivalentes de onda plana y de corte dependientes de la frecuencia, empleados para calcular la atenuación de onda P y S, respectivamente.

Los sólidos viscoelásticos equivalentes han sido empleados para realizar simulaciones numéricas de propagación de ondas considerando efectos de flujo inducido (p. ej., Rubino et al., 2011; Sidler et al., 2013), como así también para calcular coeficientes de reflexión (p. ej., Quintal et al., 2008; Ren et al., 2009; Rubino y Velis, 2011). Sin embargo, es necesario un análisis riguroso que permita evaluar el alcance de la teoría de la viscoelasticidad para representar el comportamiento de un medio poroelástico heterogéneo en general, y para calcular la atenuación debida a flujo inducido por onda en particular. Un esfuerzo reciente en esta dirección fue dado por Jänicke et al. (2015), quien derivó un proceso de homogeneización utilizando el criterio de Hill-Mandel (Hill, 1963, 1972). La razón principal por la cual es importante estudiar la hipótesis de medio viscoelástico equivalente es que, más allá de la física que gobierna las ecuaciones a escala local y de las condiciones de borde impuestas, siempre podremos definir un módulo elástico equivalente utilizando los promedios volumétricos de los campos de tensión y deformación. Sin embargo, no hay garantías de que estos módulos representen de manera adecuada el comportamiento efectivo del medio heterogéneo original desde el punto de vista de la energía elástica almacenada y de la energía disipada. De hecho, si se falla en este tipo de representación, los parámetros sísmicos derivados no serán representativos. En el caso de medios viscoelásticos, la equivalencia entre los módulos elásticos efectivos definidos en función de la energía y aquellos definidos en función de promedios de los campos de tensión y deformación está garantizada (Hill, 1963; Hashin, 1964). Sin embargo, en el planteo que estamos proponiendo aquí, el medio original es poroelástico, y el mecanismo de disipación es la fricción viscosa, mientras que dentro de la hipótesis de sólido viscoelástico equivalente la disipación es representada por viscosidad intrínseca del medio. Por lo tanto, la equivalencia entre los dos procedimientos no está garantizada. Para poner a prueba y corroborar la hipótesis de medio viscoelástico equivalente se deben desarrollar nuevas técnicas, completamente basadas en la teoría de la poroelasticidad, que permitan calcular la atenuación sísmica por flujo inducido a escalas mesoscópicas.

Si nos basamos en el concepto de disipación de la energía, el inverso del factor de calidad puede definirse de acuerdo a (p. ej., O'Connell y Budiansky, 1978; Aki y 
Richards, 1980)

$$
\frac{1}{Q^{\mathcal{M}}(\omega)}=\frac{\overline{\Delta \mathcal{P}}(\omega)}{\omega \mathcal{W}_{\max }(\omega)},
$$

donde $\overline{\Delta \mathcal{P}}$ es la potencia disipada promedio en un ciclo de onda sísmica y $\mathcal{W}_{\max }$ es el máximo de la energía elástica de deformación almacenada dentro de la muestra de prueba en el mismo ciclo. Como el flujo inducido por onda es un mecanismo de disipación, la ecuación (3.2) implica que la atenuación puede ser cuantificada calculando numéricamente $\overline{\Delta \mathcal{P}}$ y $\mathcal{W}_{\max }$ dentro del marco de la teoría de la poroelasticidad, sin suponer medio equivalente alguno para representar el comportamiento de la muestra. Por lo tanto, esta expresión puede ser utilizada para verificar la hipótesis de medio viscoelástico equivalente.

O'Connell y Budiansky (1978) han propuesto otra definición para la atenuación, que responde a

$$
\frac{1}{Q^{\mathcal{A}}(\omega)}=\frac{\overline{\Delta \mathcal{P}}(\omega)}{2 \omega \overline{\mathcal{W}}(\omega)},
$$

donde $\overline{\mathcal{W}}$ denota la energía de deformación promediada en un ciclo de onda. En el marco de la teoría de la viscoelasticidad, la ecuación (3.1) es compatible con la ecuación (3.3), es decir, $Q^{\mathcal{A}}$ y $Q^{\mathcal{D}}$ son idénticas (O'Connell y Budiansky, 1978; Masson y Pride, 2014). Además, es posible demostrar, nuevamente en el contexto de la teoría de viscoelasticidad, que $\mathcal{W}_{\text {max }} \simeq 2 \overline{\mathcal{W}}$ si $Q^{\mathcal{M}} \gtrsim 10$ (Bourbié et al., 1987). Teniendo en cuenta esta aproximación, las ecuaciones (3.2) y (3.3) son equivalentes.

En general, los estudios de flujo inducido por onda se basan en la hipótesis de medio viscoelástico equivalente (ecuación (3.1)), y la equivalencia de este procedimiento con las definiciones basadas en las ecuaciones (3.2) y (3.3) nunca ha sido explorada para materiales poroelásticos heterogéneos. En este Capítulo presentaremos una novedosa metodología para calcular la atenuación sísmica debida a flujo inducido por onda completamente basada en la teoría de la poroelasticidad.

Las definiciones y las metodologías propuestas en este Capítulo serán analizadas en escenarios clásicos de la literatura, como lo son rocas conteniendo campos de saturación tipo «patchy» binarios y medios fracturados. Esto permite evaluar el alcance de la metodología propuesta, y validar la hipótesis de medio viscoelástico equivalente en cada caso. En los Capítulos 5 y 6 de este trabajo de Tesis, haremos uso de las herramientas desarrolladas en este Capítulo para analizar el comportamiento de rocas heterogéneas saturadas por distribuciones realistas de fluidos que responden a las leyes de flujo en medios porosos.

\subsection{Experimentos oscilatorios de relajación}

El modelado numérico de la atenuación sísmica debida a flujo inducido por onda en la mesoescala es una tarea difícil. Esto se debe, principalmente, a que el proceso de difusión de presión de fluido poral sucede a escalas mucho menores que la longitud de onda (p. ej., Rubino et al., 2011). Por lo tanto, al discretizar el medio, es necesario considerar celdas computacionales suficientemente pequeñas como para representar de 
manera adecuada las heterogeneidades mesoscópicas y el respectivo proceso de difusión. A su vez, se debe considerar un dominio de resolución suficientemente extenso como para poder observar el decaimiento de amplitud de la onda sísmica en el rango de frecuencias de interés. Cumplir, de manera simultánea, estos dos requerimientos da lugar a un sistema de ecuaciones cuya resolución tiene un alto costo computacional (Rubino et al., 2009).

Con la intención de proveer una solución a este problema, Rubino et al. (2009, 2013 b) propusieron un procedimiento numérico de homogeneización basado en la resolución de las ecuaciones de consolidación de Biot (2.46)-(2.47), en conjunto con las relaciones constitutivas (2.16)-(2.17). Este consiste en la aplicación de un experimento numérico de relajación a una muestra de roca bidimensional que contiene una distribución de heterogeneidades de mesoescala representativa del medio de interés $(\mathrm{REV})^{1}$. Los promedios volumétricos de los campos de tensión y deformación resultantes permiten estimar la atenuación y la dispersión por flujo mesoscópico haciendo uso de la hipótesis de medio viscoelástico equivalente. Como las dimensiones de la muestra pueden ser mucho menores que la longitud de onda predominante $\lambda$, se evita el alto costo computacional que supone modelar un proceso de propagación.

Como se verá a continuación, los campos de desplazamiento de la matriz sólida y del fluido, en respuesta al experimento considerado, pueden emplearse para calcular la atenuación del medio a través de consideraciones energéticas, sin recurrir a la hipótesis de medio viscoelástico equivalente.

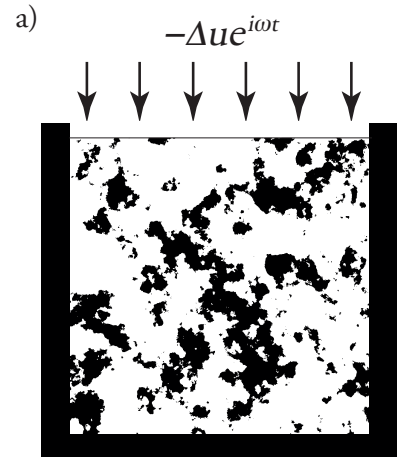

b)

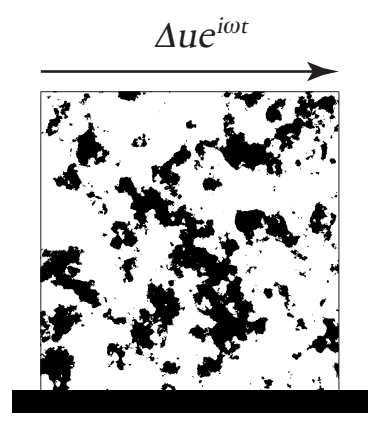

Figura 3.1. Ilustración esquemática de las experimentos numéricos de relajación empleados para determinar las propiedades de las ondas a) P y b) S. Se aplica un desplazamiento $\Delta u$ a lo largo del límite superior de las muestras, emulando el campo de tensiones y deformaciones asociados con el paso de una onda sísmica.

Para obtener la respuesta de la muestra de roca seleccionada ante la deformación generada por el paso de una onda compresional $\mathrm{P}$, aplicamos un desplazamiento sólido vertical y armónico en tiempo, de la forma $\Delta u e^{i \omega t}$ a lo largo del borde superior de la misma (Figura 3.1a). No se aplican fuerzas tangenciales a los bordes de la muestra. Además, el sólido está imposibilitado de moverse verticalmente en la base de la muestra y no posee desplazamientos horizontales en los límites laterales. El fluido se encuentra completamente contenido dentro de la muestra, es decir, los bordes están sellados hidráulicamente.

\footnotetext{
${ }^{1}$ El lector no debe confundir este REV con el aquel empleado en el marco de la formulación de medios continuos en el Capítulo 2.
} 
Sea $\Omega=\left(0, L_{x}\right) \times\left(0, L_{y}\right)$ el dominio que representa la muestra de prueba, con frontera $\Gamma$, la cual está compuesta por $\Gamma=\Gamma^{L} \cup \Gamma^{B} \cup \Gamma^{R} \cup \Gamma^{T}$, donde

$$
\begin{gathered}
\Gamma^{L}=\{(x, y) \in \Omega: x=0\}, \\
\Gamma^{R}=\left\{(x, y) \in \Omega: x=L_{x}\right\}, \\
\Gamma^{B}=\{(x, y) \in \Omega: y=0\}, \\
\Gamma^{T}=\left\{(x, y) \in \Omega: y=L_{y}\right\} .
\end{gathered}
$$

Para estimar la respuesta de la muestra de roca sujeta al experimento de relajación consideramos entonces las siguientes condiciones de borde

$$
\begin{gathered}
\hat{\mathbf{u}} \cdot \boldsymbol{\nu}=-\Delta u, \quad(x, y) \in \Gamma^{T}, \\
\hat{\mathbf{u}} \cdot \boldsymbol{\nu}=0, \quad(x, y) \in \Gamma^{L} \cup \Gamma^{R} \cup \Gamma^{B}, \\
(\hat{\boldsymbol{\sigma}} \cdot \boldsymbol{\nu})^{\mathrm{T}} \cdot \boldsymbol{\chi}=0, \quad(x, y) \in \Gamma, \\
\hat{\mathbf{w}} \cdot \boldsymbol{\nu}=0, \quad(x, y) \in \Gamma .
\end{gathered}
$$

Donde $\boldsymbol{\nu}$ y $\chi$ denotan la normal saliente unitaria y la tangente unitaria sobre $\Gamma$, respectivamente. De esta forma, $\{\boldsymbol{\nu}, \boldsymbol{\chi}\}$ es un sistema ortonormal sobre $\Gamma$.

Por otro lado, en el caso de una onda $\mathrm{S}$, imponemos un desplazamiento horizontal de la forma $\Delta u e^{i \omega t}$ a lo largo del límite superior de la muestra (Figura 3.1b). No se aplican fuerzas normales en los bordes de la muestra e imponemos condiciones de desplazamiento vertical nulo en los bordes laterales y desplazamiento horizontal nulo en la base de la muestra. Además, los bordes de la muestra están sellados hidráulicamente. El juego de condiciones de borde empleado en el experimento de relajación de corte responde a

$$
\begin{gathered}
\hat{\mathbf{u}} \cdot \boldsymbol{\chi}=\Delta u, \quad(x, y) \in \Gamma^{T}, \\
\hat{\mathbf{u}} \cdot \boldsymbol{\chi}=0, \quad(x, y) \in \Gamma^{L} \cup \Gamma^{R} \cup \Gamma^{B}, \\
(\hat{\boldsymbol{\sigma}} \cdot \boldsymbol{\nu})^{\mathrm{T}} \cdot \boldsymbol{\nu}=0, \quad(x, y) \in \Gamma, \\
\hat{\mathbf{w}} \cdot \boldsymbol{\nu}=0, \quad(x, y) \in \Gamma .
\end{gathered}
$$




\subsection{Modelado de la atenuación sísmica utilizando consideraciones energéticas}

El comportamiento de una roca heterogénea sujeta a un experimento oscilatorio de relajación permite obtener estimaciones de la atenuación sísmica utilizando consideraciones energéticas mediante las definiciones (3.2) y (3.3). Para realizar estos cálculos es necesario contar con estimaciones de la potencia disipada y la energía elástica almacenada en la muestra. A continuación describiremos el procedimiento para obtener, de forma numérica, estos parámetros para una roca poroelástica heterogénea.

\subsubsection{Potencia disipada promedio}

La potencia disipada cuantifica la energía que se pierde en el sistema por unidad de tiempo. En el caso de materiales poroelásticos, la energía de disipación se debe a la fricción viscosa que ocurre en el fluido poral y depende de la función de disipación por unidad de volumen $\mathcal{D}$ (ecuación (2.23)). La contribución local a la potencia disipada en función del tiempo responde a (Biot, 1962; Bourbié et al., 1987)

$$
\begin{aligned}
\mathrm{d} \mathcal{P}(\mathrm{t}) & =2 \mathcal{D} \mathrm{dV} \\
& =\dot{\mathbf{w}}^{\mathrm{T}} \frac{\eta}{\kappa} \dot{\mathbf{w}} \mathrm{dV}
\end{aligned}
$$

Procedamos entonces a calcular el promedio de $\mathrm{d} \mathcal{P}(\mathrm{t})$ sobre un período $2 \pi / \omega$. Para ello es importante resaltar que consideramos una perturbación armónica del campo de deformación en respuesta al experimento oscilatorio, es decir, $\mathbf{w}(t)=\Re\left\{\hat{\mathbf{w}} e^{i \omega t}\right\} \mathrm{y}$ que, en el dominio del espacio y la frecuencia, $\hat{\mathbf{w}}=i \omega \hat{\mathbf{w}}$. Luego, podemos expresar el promedio de $\mathrm{d} \mathcal{P}(\mathrm{t})$ sobre un período de oscilación de acuerdo a

$$
\overline{\mathrm{d} \mathcal{P}}(\omega)=\frac{\omega}{2 \pi} \int_{0}^{\frac{2 \pi}{\omega}} \Re\left\{i \omega \hat{\mathbf{w}}^{T} e^{i \omega t}\right\} \Re\{\mathbf{K}\} \Re\left\{i \omega \hat{\mathbf{w}} e^{i \omega t}\right\} \mathrm{dt} \mathrm{dV},
$$

donde $\mathbf{K}=\frac{\eta}{\kappa} \mathbf{I}$. Las propiedades de promediación en variables complejas dictan que dados un vector A, cuyas componentes son armónicas y complejas, y una matriz simétrica $\mathbb{D}$, se puede verificar que (p. ej., Rubino et al., 2006)

$$
\frac{\omega}{2 \pi} \int_{0}^{\frac{2 \pi}{\omega}} \Re\left\{\mathbf{A}^{T}\right\} \Re\{\mathbb{D}\} \Re\{\mathbf{A}\} \mathrm{dt}=\frac{1}{2} \Re\left\{\mathbf{A}^{\mathrm{T}} \mathbb{D} \mathbf{A}^{*}\right\},
$$

donde $*$ denota el complejo conjugado. Si empleamos este resultado en la ecuación (3.17), obtenemos

$$
\overline{\mathrm{d} \mathcal{P}}(\omega)=\frac{1}{2} \Re\left\{(i \omega \hat{\mathbf{w}})^{T} \mathbf{K}(i \omega \hat{\mathbf{w}})^{*}\right\} \mathrm{dV}=\frac{\eta}{2 \kappa} \Re\left\{(\mathrm{i} \omega \hat{\mathbf{w}})^{\mathrm{T}}(\mathrm{i} \omega \hat{\mathbf{w}})^{*}\right\} \mathrm{dV} .
$$

Luego, utilizando que $(i \hat{\mathbf{w}})^{T}(i \hat{\mathbf{w}})^{*}=\|\hat{\mathbf{w}}\|^{2}$, e integrando sobre el volumen de estudio, obtenemos la potencia disipada promediada en un período (Johnson, 2001; Müller y Rothert, 2006)

$$
\overline{\Delta \mathcal{P}}(\omega)=\frac{\omega^{2}}{2} \int_{\Omega} \frac{\eta}{\kappa}\|\hat{\mathbf{w}}\|^{2} \mathrm{dV}
$$


$\mathrm{Al}$ resolver de forma numérica las ecuaciones cuasiestáticas de Biot (ecuaciones (2.46) a $(2.47)$ ), discretizamos el dominio computacional $\Omega$ en $N_{x} \times N_{y}$ elementos rectangulares $\Omega_{k l}$ de área $\delta^{2}$. El procedimiento numérico permite obtener el desplazamiento relativo del fluido $(\hat{\mathbf{w}})_{k l}$ en cada elemento $\Omega_{k l}$, y entonces, la potencia media disipada en esa región está dada por

$$
\overline{\Delta \mathcal{P}_{k l}}(\omega)=\frac{\omega^{2}}{2}\left(\frac{\eta}{\kappa}\|\hat{\mathbf{w}}\|^{2}\right)_{k l} \delta^{2} .
$$

Luego, la potencia media disipada en toda la muestra se obtiene sumando las contribuciones locales de cada región

$$
\overline{\Delta \mathcal{P}}(\omega)=\sum_{k=1}^{N_{x}} \sum_{l=1}^{N_{y}} \overline{\Delta \mathcal{P}_{k l}}(\omega) .
$$

Si se desea emplear las ecuaciones (3.2) y (3.3) para calcular la atenuación sísmica, además de la potencia media disipada, es necesario poseer una estimación del máximo y del promedio de la energía elástica de deformación dentro de la muestra estudiada. A continuación abordaremos este tópico, empleando definiciones de la teoría de la poroelasticidad.

\subsubsection{Energía elástica de deformación}

Para una onda monocromática de frecuencia $\omega$, la energía de deformación acumulada en el elemento $\Omega_{k l}$ en función del tiempo $\mathcal{W}_{k l}(t)$ está dada por la ecuación (2.18), multiplicada por el área de la celda $\delta^{2}$. Para computar la energía elástica máxima sumamos los valores de $\mathcal{W}_{k l}(t) \delta^{2}$ sobre el dominio discretizado y determinamos numéricamente el máximo en el dominio del tiempo

$$
\mathcal{W}_{\text {max }}(\omega)=\operatorname{máx}_{0 \leq t \leq \frac{2 \pi}{\omega}}\left\{\sum_{k=1}^{N_{x}} \sum_{l=1}^{N_{y}} \mathcal{W}_{k l}(t) \delta^{2}\right\} .
$$

Por otro lado, para estimar la energía de deformación promedio sobre un ciclo de onda utilizamos, tal como hemos hecho en el caso de la potencia disipada, propiedades de promediación aplicadas a variables temporales, armónicas, y complejas (p. ej., Rubino et al., 2006). Como resultado, la energía de deformación promedio sobre un ciclo de onda, en un elemento $\Omega_{k l}$ puede expresarse de acuerdo a

$$
\overline{\mathcal{W}_{k l}}(\omega)=\frac{1}{4} \Re\left\{\hat{\boldsymbol{\sigma}}: \hat{\boldsymbol{\epsilon}}^{*}+\hat{p}_{f} \hat{\zeta}^{*}\right\}_{k l} \delta^{2} .
$$

Los valores de las componentes de los tensores de tensión y deformación, tanto como la presión y el cambio en el contenido de fluido, son determinados utilizando las relaciones constitutivas una vez que se obtienen los desplazamientos del sólido y del fluido por medio del experimento oscilatorio de relajación previamente descripto.

La energía elástica de deformación promedio sobre la muestra de prueba está dada por

$$
\overline{\mathcal{W}}(\omega)=\sum_{k=1}^{N_{x}} \sum_{l=1}^{N_{y}} \overline{\mathcal{W}_{k l}}(\omega) .
$$

Con esta información podemos proceder a calcular la atenuación por medio de consideraciones energéticas poroelásticas. 


\subsection{Atenuación global y contribución local}

Utilizando las ecuaciones (3.2) y (3.22) podemos expresar

$$
\frac{1}{Q^{\mathcal{M}}(\omega)}=\frac{\sum_{k=1}^{N_{x}} \sum_{l=1}^{N_{y}} \overline{\Delta \mathcal{P}_{k l}}(\omega)}{\omega \mathcal{W}_{\max }(\omega)}
$$

con $\overline{\Delta \mathcal{P}_{k l}}(\omega)$ y $\mathcal{W}_{\text {max }}(\omega)$ dados por las ecuaciones (3.21) y (3.23), respectivamente. Por otro lado, considerando las ecuaciones (3.3) y(3.22), tenemos

$$
\frac{1}{Q^{\mathcal{A}}(\omega)}=\frac{\sum_{k=1}^{N_{x}} \sum_{l=1}^{N_{y}} \overline{\Delta \mathcal{P}_{k l}}(\omega)}{2 \omega \overline{\mathcal{W}}(\omega)}
$$

donde $\overline{\mathcal{W}}(\omega)$ responde a la ecuación (3.25).

Como indica la ecuación (3.27), se puede definir, para una frecuencia determinada $\omega$, la contribución local a la atenuación sísmica. Esto se logra reescribiendo la ecuación (3.27) de acuerdo a

$$
\frac{1}{Q^{\mathcal{A}}(\omega)}=\sum_{k=1}^{N_{x}} \sum_{l=1}^{N_{y}} q_{k l}^{-1}(\omega) \delta^{2}
$$

donde

$$
q_{k l}^{-1}(\omega)=\frac{\overline{\Delta \mathcal{P}_{k l}}(\omega)}{2 \delta^{2} \omega \overline{\mathcal{W}}(\omega)},
$$

es la contribución del elemento $\Omega_{k l}$ a la atenuación sísmica global de la roca de prueba por unidad de área. Este parámetro permite observar directamente cómo contribuyen las heterogeneidades al proceso de disipación de flujo inducido por onda. Es importante notar que la ecuación (3.29) no puede obtenerse mediante los procedimientos basados en la hipótesis de medio viscoelástico equivalente.

A continuación describiremos el procedimiento de cálculo de la atenuación sísmica por medio de la hipótesis de medio viscoelástico equivalente, el cual hace uso de estimaciones del módulo de onda plana y módulo de corte equivalentes empleando promedios volumétricos de componentes del tensor de tensión y deformación resultantes del experimento oscilatorio de relajación.

\subsection{Hipótesis de medio viscoelástico equivalente}

El procedimiento estándar para obtener la atenuación sísmica debida a flujo inducido asume que el medio poroelástico estudiado puede ser representado por un medio viscoelástico homogéneo equivalente. Luego, cuando una roca es sometida a un experimento de relajación, es posible obtener los módulos de onda plana y de corte del medio viscoelástico equivalente considerando el cociente entre el promedio volumétrico de ciertas componentes del tensor de tensión y del tensor de deformación resultantes. 
Asumiendo que los promedios volumétricos de las tensiones y deformaciones están relacionados a través de las relaciones constitutivas para sólidos viscoelásticos, podemos definir un módulo de onda plana equivalente

$$
M_{c}(\omega)=\frac{\left\langle\hat{\sigma}_{y y}(\omega)\right\rangle}{\left\langle\hat{\epsilon}_{y y}(\omega)\right\rangle},
$$

donde $\left\langle\hat{\sigma}_{y y}(\omega)\right\rangle$ y $\left\langle\hat{\epsilon}_{y y}(\omega)\right\rangle$ denotan los promedios volumétricos de las componentes verticales de los campos de tensión y deformación que resultan del experimento oscilatorio de relajación ilustrado en la Figura 3.1(a). Análogamente, podemos definir un módulo de corte equivalente de acuerdo a

$$
\mu_{c}(\omega)=\frac{1}{2} \frac{\left\langle\hat{\sigma}_{x y}(\omega)\right\rangle}{\left\langle\hat{\epsilon}_{x y}(\omega)\right\rangle}
$$

donde $\left\langle\hat{\sigma}_{x y}(\omega)\right\rangle$ y $\left\langle\hat{\epsilon}_{x y}(\omega)\right\rangle$ son los promedios volumétricos de las componentes de corte de los campos de tensión y deformación que resultan del experimento oscilatorio ilustrado en la Figura 3.1(b). Este procedimiento, representado en las ecuaciones (3.30) y (3.31), es en sí un proceso «upscaling», ya que permite analizar las propiedades efectivas del medio estudiado a una escala mayor.

Finalmente, el inverso del factor de calidad se deriva de los módulos equivalentes empleando la ecuación (3.1). Es importante notar que, en este contexto, no es posible calcular las contribuciones locales de las diferentes regiones de la roca a la atenuación total.

Para estudiar la relación entre las definiciones (3.1), (3.2), y (3.3), calcularemos las curvas de atenuación en función de la frecuencia para una serie de escenarios clásicos de la literatura, tales como, por ejemplo, medios periódicos estratificados (teoría de White), medios binarios saturados con distribuciones estocásticas de fluidos, y medios fracturados. A su vez, exploraremos la contribución local a la atenuación global en el interior de dichos medios.

\subsection{Modelo 1-D de saturación «patchy»}

A continuación analizaremos un escenario clásico de estudio de los efectos del flujo mesoscópico sobre las ondas sísmicas, comúnmente denominado medio de White. Este posee una geometría simple (1-D) y la solución al sistema de ecuaciones puede expresarse analíticamente. Las secciones anteriores han permitido obtener expresiones para calcular las atenuaciones, ya sea por medio de la hipótesis de medio viscoelástico equivalente o bien empleando consideraciones energéticas, y su contribución local en un medio 2-D. Estas expresiones pueden emplearse en medios estratificados 1-D, considerando únicamente desplazamientos en la dirección perpendicular a las capas.

\subsubsection{Medios periódicos estratificados: Teoría de White}

El problema de obtener la atenuación y dispersión sísmica de un medio compuesto por capas planas paralelas y periódicas saturadas de manera alternada con gas y agua fue, en primer instancia, abordado por White et al. (1975). Desde un punto de vista 
hidrológico, los modelos de roca homogénea saturada por campos binarios de fluidos inmiscibles son escenarios inestables. Es decir, debido a efectos gravitatorios, el gas tendería a migrar por flotabilidad y, a su vez, las transiciones entre gas y agua tenderían a difundirse debido a gradientes en la presión capilar. Por lo cual, los modelos de roca saturada por campos binarios de fluidos inmiscibles no son realistas. La representatividad de las firmas sísmicas derivadas de este tipo de modelos es un problema que será abordado en el Capítulo 6 de este trabajo de Tesis. A pesar de su simplicidad, el modelo de White continua siendo, en la actualidad, uno de los modelos más estudiados en el contexto de la disipación sísmica por flujo inducido por onda en la mesoescala (p. ej., Norris, 1993; Gurevich y Lopatnikov, 1995; Carcione y Picotti, 2006; Vogelaar y Smeulders, 2007; Qi et al., 2014b). A continuación analizaremos la validez de la hipótesis de medio viscoelástico equivalente para el modelo de White, empleando la metodología poroelástica desarrollada. A su vez, utilizaremos la contribución local a la atenuación para explorar las características internas del proceso de disipación en dicho modelo.

El modelo de White explora la disipación en un medio poroso homogéneo, saturado de forma alternada por dos fluidos con compresibilidades diferentes al ser atravesado transversalmente por una onda sísmica compresional. La Figura 3.2 ilustra el medio periódico estratificado correspondiente, utilizando colores gris y blanco para esquematizar las regiones completamente saturadas con agua y gas, respectivamente. Para calcular los campos de desplazamiento del sólido $\hat{\mathbf{u}}=[0, \hat{\mathrm{u}}(y)]$ y del fluido $\hat{\mathbf{w}}=[0, \hat{\mathrm{w}}(y)]$, asociados con el campo de deformación de una onda sísmica compresional, consideraremos un volumen elemental representativo (REV) del medio. Debido a la simetría inherente del modelo, estudiamos la respuesta del medio considerando un subdominio compuesto por dos semicapas, como se ilustra en la Figura 3.2b.

Resolvemos las ecuaciones de consolidación de Biot (1941) en la muestra seleccionada, empleando condiciones de borde apropiadas. Es importante recalcar que este cálculo puede ser llevado a cabo de forma numérica empleando las condiciones de borde descriptas en la Sección 3.2. Sin embargo, una de las características más notables del modelo de White es que el sistema de ecuaciones resultante posee una solución analítica. Para este caso 1-D particular, imponemos un desplazamiento armónico y dependiente del tiempo $-\Delta u e^{i \omega t}$ en el borde superior del subdominio compuesto por dos semicapas, emulando la deformación causada por el paso de una onda sísmica compresional (Figura 3.2b). Además, debido a la simetría del sistema de capas paralelas, se asume que el flujo es nulo en el techo y que tanto el flujo como el desplazamiento de la fase sólida son nulos en la base de la muestra. Luego, en el dominio del espacio y la frecuencia, las condiciones de borde responden a

$$
\begin{aligned}
& \hat{\mathrm{u}}(y)=-\Delta u, \quad y=L, \\
& \hat{\mathrm{w}}(y)=0, \quad y=L, \\
& \hat{\mathrm{W}}(y)=0, \quad y=0, \\
& \hat{\mathrm{u}}(y)=0, \quad y=0 .
\end{aligned}
$$

donde el eje de coordenadas y se define en la dirección perpendicular a la estratificación, de manera que el origen se encuentre en la base de la muestra considerada. A su vez, $L$ denota el espesor total de la muestra. Además, $L=L_{1}+L_{2}$, con $L_{1}$ el espesor de la semicapa con gas, y $L_{2}$ el espesor de la semicapa con agua. Es necesario, para obtener la 
a)

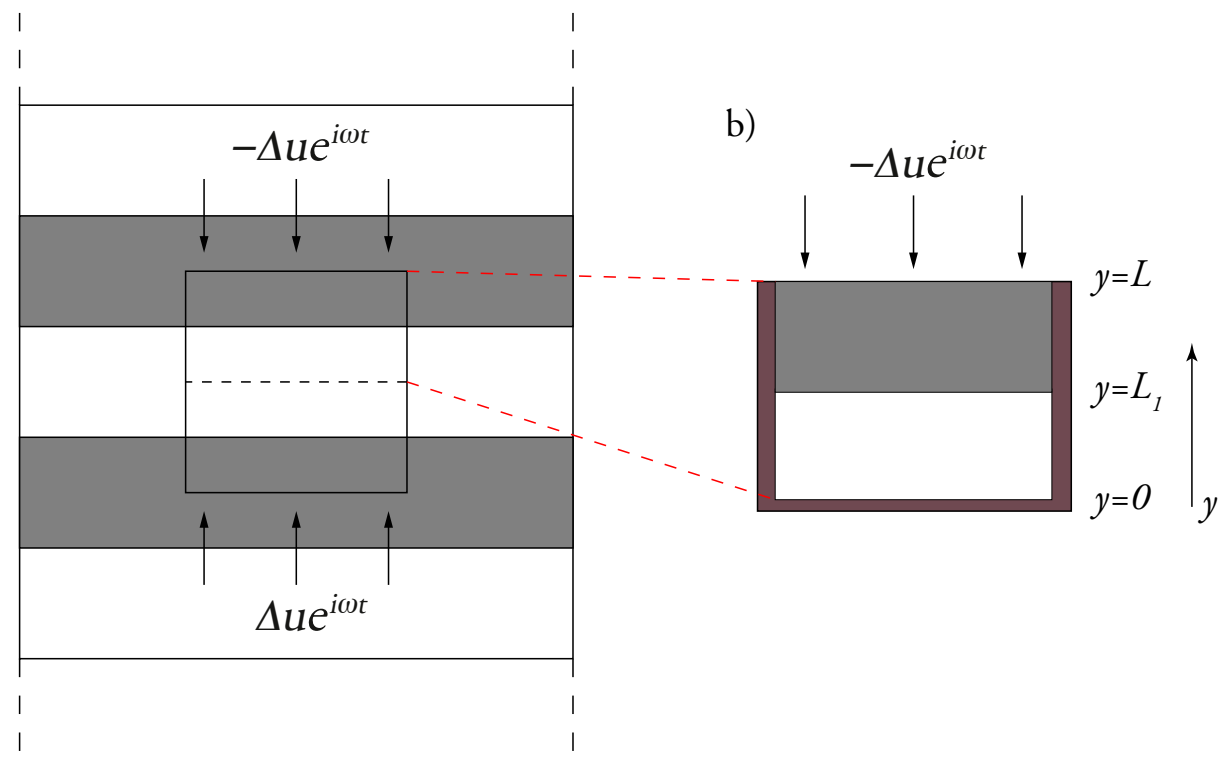

Figura 3.2. (a) Ilustración esquemática del modelo de capas paralelas de White. El rectángulo de bordes negros delimita un posible REV del medio en cuestión. Como consecuencia de la simetría inherente del sistema, el comportamiento del medio puede ser explorado utilizando la mitad superior (b) de este rectángulo, donde $y$ varía de 0 a $L$.

solución analítica del modelo de White, imponer condiciones adicionales en la interfase entre ambas semicapas. En este sentido, imponemos continuidad del desplazamiento del fluido y del sólido, de manera que

$$
\begin{aligned}
\hat{u}_{1}\left(L_{1}\right) & =\hat{u}_{2}\left(L_{1}\right), \\
\hat{w}_{1}\left(L_{1}\right) & =\hat{w}_{2}\left(L_{1}\right),
\end{aligned}
$$

donde los subíndices 1 y 2 denotan los parámetros correspondientes a las semicapas saturadas con gas y con agua, respectivamente. Además, imponemos continuidad de las presiones y tensiones

$$
\begin{gathered}
p_{1, f}\left(L_{1}\right)=p_{2, f}\left(L_{1}\right), \\
\sigma_{1, y y}\left(L_{1}\right)=\sigma_{2, y y}\left(L_{1}\right) .
\end{gathered}
$$

Es importante notar que estamos considerando una condición de «poro abierto», es decir, que existe una buena comunicación hidráulica entre ambas capas. En este sentido, Qi et al. (2014b) propuso una condición alternativa, que modela el posible salto de presión entre ambas capas considerando que $p_{1, f}\left(L_{1}\right)-p_{2, f}\left(L_{1}\right)=-\mathrm{Z} \dot{w}(y)$, donde $\mathrm{Z}$ es un coeficiente de resistencia. En este trabajo, sin embargo, hemos seguido el procedimiento estándar, asumiendo $\mathrm{Z}=0$.

Obtengamos entonces la solución analítica del modelo de White, para explorar la atenuación por flujo mesoscópico. Para un medio homogéneo, considerando únicamente desplazamientos en la dirección del eje $y$, las ecuaciones de movimiento y las relaciones 
constitutivas (ecuaciones (2.16)-(2.17) y (2.46)-(2.47)) pueden combinarse para obtener

$$
\frac{\partial^{2} \hat{w}(y)}{\partial y^{2}} D=i \omega \hat{w}(y)
$$

donde $D$ es la difusividad del medio, dada por la ecuación (2.49). Para resolver esta ecuación en cada capa, se propone que los desplazamientos relativos del fluido $\hat{w}(y)$ responden a una combinación de exponenciales, es decir,

$$
\begin{array}{ll}
\hat{w}_{1}(y)=\mathbb{A}_{1} e^{k_{1} y}+\mathbb{B}_{1} e^{-k_{1} y}, & y \in\left[0, L_{1}\right], \\
\hat{w}_{2}(y)=\mathbb{A}_{2} e^{k_{2} y}+\mathbb{B}_{2} e^{-k_{2} y}, & y \in\left[L_{1}, L\right],
\end{array}
$$

donde

$$
k_{j}=\sqrt{\frac{i \omega}{D_{j}}}, j=1,2,
$$

es el número de onda de la onda lenta de Biot en cada semicapa. Los parámetros $\mathbb{A}_{j}$, $\mathbb{B}_{j}$, con $j=1,2$, son constantes a determinar. Es importante notar que, debido a la ecuación de equilibrio (2.46), la tensión $\hat{\sigma}_{j, y y}$ es constante en cada capa.

Las ecuaciones constitutivas (2.16) y (2.17) permiten obtener las siguientes expresiones para la presión del fluido poral y el desplazamiento de la fase sólida en cada capa

$$
\begin{gathered}
\hat{p}_{j, f}(y)=-\beta_{s, j} \hat{\sigma}_{j, y y}+\left(\beta_{s, j}^{2} H_{j}-M_{j}\right) \frac{\partial \hat{w}_{j}(y)}{\partial y}, \\
\hat{u}_{j}(y)=\frac{\hat{\sigma}_{j, y y}}{H_{j}} y-\beta_{s, j} \hat{w}_{j}(y)+\mathbb{E}_{j}, \quad j=1,2,
\end{gathered}
$$

donde $\mathbb{E}_{1}$ y $\mathbb{E}_{2}$ son constantes de integración, y $\beta_{s, j}=\alpha_{j} M_{j} / H_{j}$ son los coeficientes de Skempton 1-D asociados a cada semicapa. El coeficiente de Skempton para cada capa se define como el cociente entre la variación de la presión del fluido y la tensión aplicada en estado «undrained», es decir $\beta_{s, j} \equiv-\partial \hat{p}_{j, f} /\left.\partial \hat{\sigma}_{y y}\right|_{\xi=0}$ (p. ej., Wang, 2017). Por lo cual, este parámetro mide cómo aumenta la presión de fluido frente a una tensión aplicada (Wang, 2017).

Para encontrar las expresiones de los campos de desplazamiento en función de la posición es preciso determinar los valores de las constantes $\mathbb{A}_{j}, \mathbb{B}_{j}, \mathbb{E}_{j}$ y $\hat{\sigma}_{j, y y}$. Para ello, emplearemos las condiciones de borde (3.32) a (3.35), y las condiciones de continuidad (3.36) a (3.39).

Las constantes $\mathbb{A}_{j}$ y $\mathbb{B}_{j}$ pueden vincularse analizando los valores de $\hat{w}_{j}(y)$ en los límites superior e inferior de la muestra explorada empleando las condiciones de borde, es decir,

$$
\begin{aligned}
\hat{w}_{1}(0)=\mathbb{A}_{1} e^{k_{1} 0}+\mathbb{B}_{1} e^{-k_{1} 0} & =0, \\
\mathbb{A}_{1} & =-\mathbb{B}_{1}, \\
\hat{w}_{2}(L)=\mathbb{A}_{2} e^{k_{2} L}+\mathbb{B}_{2} e^{-k_{2} L} & =0, \\
\mathbb{A}_{2} & =-\mathbb{B}_{2} e^{-2 L k_{2}} .
\end{aligned}
$$


Por lo cual, las expresiones para el desplazamiento del fluido en cada semicapa de la muestra considerada responden a

$$
\begin{array}{rr}
\hat{w}_{1}(y)=\mathbb{A}_{1}\left(e^{k_{1} y}-e^{-k_{1} y}\right), & y \in\left[0, L_{1}\right], \\
\hat{w}_{2}(y)=\mathbb{B}_{2}\left(e^{-k_{2} y}-e^{k_{2}(y-2 L)}\right), & y \in\left[L_{1}, L\right] .
\end{array}
$$

Por otro lado, las constantes $\mathbb{E}_{j}$ pueden calcularse a través de la ecuación (3.45), evaluando $\hat{u}_{j}(y)$, en los bordes superior e inferior de la muestra estudiada, es decir,

$$
\begin{aligned}
\hat{u}_{1}(0)=\mathbb{E}_{1} & =0, \\
\hat{u}_{2}(L)=\frac{\hat{\sigma}_{2, y y}}{H_{2}} L+\mathbb{E}_{2} & =-\Delta u, \\
\mathbb{E}_{2} & =-\Delta u-\frac{\hat{\sigma}_{2, y y}}{H_{2}} L .
\end{aligned}
$$

Entonces, las expresiones para el desplazamiento de la fase sólida responden a

$$
\begin{array}{r}
\hat{u}_{1}(y)=\frac{\hat{\sigma}_{1, y y}}{H_{1}} y-\beta_{s, 1} \hat{w}_{1}(y), \quad y \in\left[0, L_{1}\right], \\
\hat{u}_{2}(y)=-\Delta u+\frac{\hat{\sigma}_{2, y y}}{H_{2}}(y-L)-\beta_{s, 2} \hat{w}_{2}(y), \quad y \in\left[L_{1}, L\right] .
\end{array}
$$

Para determinar las constantes restantes, $\mathbb{A}_{1}$ y $\mathbb{B}_{2}$, así como el valor asociado a los esfuerzos $\hat{\sigma}_{j, y y}$, se emplean las ecuaciones (3.36)-(3.39). En primer lugar, empleando la ecuación (3.39), observamos que la tensión es constante en toda el dominio $\hat{\sigma}_{j, y y} \equiv \hat{\sigma}_{y y}$. Luego, evaluando las presiones y desplazamientos en la interfase $y=L_{1}$ se obtiene

$$
\begin{gathered}
\mathbb{A}_{1}=\left(e^{k_{1} L_{1}}-e^{-k_{1} L_{1}}\right)^{-1} \frac{\hat{\sigma}_{y y}\left[\beta_{s, 2}-\beta_{s, 1}\right]}{i \omega \sum_{j=1}^{2} \frac{\eta_{j}}{k_{j} \kappa_{j}} \operatorname{coth} k_{j} L_{j}}, \\
\mathbb{B}_{2}=\left(e^{-k_{2} L_{1}}-e^{k_{2}\left(L_{1}-2 L\right)}\right)^{-1} \frac{\hat{\sigma}_{y y}\left[\beta_{s, 2}-\beta_{s, 1}\right]}{i \omega \sum_{j=1}^{2} \frac{\eta_{j}}{k_{j} \kappa_{j}} \operatorname{coth} k_{j} L_{j}}, \\
\hat{\sigma}_{y y}=-\Delta u\left[\left(\frac{L_{1}}{H_{1}}+\frac{L_{2}}{H_{2}}\right)+\frac{\left[\beta_{s, 2}-\beta_{s, 1}\right]^{2}}{i \omega \sum_{j=1}^{2} \frac{\eta_{j}}{k_{j} \kappa_{j}} \operatorname{coth} k_{j} L_{j}}\right]^{-1} .
\end{gathered}
$$

De esta manera, se obtienen los desplazamientos del sólido y del fluido $\hat{w}(y)$ y $\hat{u}(y)$ en cada capa (ecuaciones (3.50), (3.51),(3.55), y (3.56)). Empleando sus expresiones, es posible calcular de forma directa la potencia disipada y la energía almacenada a través de las ecuaciones (3.25),(3.23) y (3.22). Luego, es posible calcular la atenuación empleando las definiciones poroelásticas $1 / Q_{p}^{\mathcal{M}}$ y $1 / Q_{p}^{\mathcal{A}}$, ecuaciones (3.2) y (3.3).

Por otro lado, para calcular $1 / Q_{p}^{\mathcal{D}}$ empleando medios viscoelásticos equivalentes (ecuación (3.1)) es necesario contar con una estimación del módulo de onda plana viscoelástico equivalente del sistema $M_{c}(\omega)$. Este puede ser calculado utilizando la ecuación (3.30), donde $\left\langle\hat{\epsilon}_{y y}\right\rangle$ responde en este caso a

$$
\left\langle\hat{\epsilon}_{y y}\right\rangle=\frac{1}{L} \int_{0}^{L} \frac{\partial \hat{u}(y)}{\partial y} \mathrm{~d} y=\frac{1}{L}\left(\hat{u}_{2}(L)-\hat{u}_{1}(0)\right)=-\Delta u / L
$$


Tabla 3.1. Propiedades físicas de los materiales sólidos y fluidos empleados en las simulaciones numéricas (Rubino et al., 2011, 2014).

\begin{tabular}{lcclcc}
\hline & Arenisca Utsira & Arenisca & & Agua & $\mathrm{CO}_{2}$ \\
\hline$K_{s}$ & $36.9 \mathrm{GPa}$ & $37 \mathrm{GPa}$ & $K_{f}$ & $2.3 \mathrm{GPa}$ & $0.0229 \mathrm{GPa}$ \\
$\rho_{s}$ & $2650 \mathrm{Kg} / \mathrm{m}^{3}$ & $2650 \mathrm{Kg} / \mathrm{m}^{3}$ & $\rho_{f}$ & $1090 \mathrm{Kg} / \mathrm{m}^{3}$ & $693 \mathrm{Kg} / \mathrm{m}^{3}$ \\
$\phi$ & 0.37 & 0.1 & $\eta$ & $0.001 \mathrm{~Pa} . \mathrm{s}$ & $1.56 \times 10^{-5} \mathrm{~Pa} . \mathrm{s}$ \\
$K_{m}$ & $2.68 \mathrm{GPa}$ & $26 \mathrm{GPa}$ & & & \\
$\mu_{m}$ & $0.857 \mathrm{GPa}$ & $31 \mathrm{GPa}$ & & & \\
$\kappa$ & $1 \mathrm{D}$ & $10^{-5} \mathrm{D}$ & & & \\
\hline
\end{tabular}

$\mathrm{y}\left\langle\hat{\sigma}_{y y}\right\rangle=\hat{\sigma}_{y y}$. Luego, el módulo de onda plana para el modelo de White responde a

$$
M_{c}(\omega)=\left[\left(\frac{L_{1}}{L H_{1}}+\frac{L_{2}}{L H_{2}}\right)+\frac{\left[\beta_{s, 2}-\beta_{s, 1}\right]^{2}}{i \omega L \sum_{j=1}^{2} \frac{\eta_{j}}{k_{j} \kappa_{j}} \operatorname{coth} k_{j} L_{j}}\right]^{-1}
$$

Por lo cual, el módulo de onda plana efectivo para el modelo de White depende del contraste entre los módulos de onda plana «undrained» $H_{1}$ y $H_{2}$, pesados por la longitud relativa de cada semicapa $L_{1} / L$ y $L_{2} / L$, y del contraste de los coeficientes de Skempton $\beta_{s, 1}$ y $\beta_{s, 2}$.

A continuación, compararemos estas tres definiciones de atenuación sísmica $\left(1 / Q_{p}^{\mathcal{D}}\right.$, $1 / Q_{p}^{\mathcal{M}}$, y $\left.1 / Q_{p}^{\mathcal{A}}\right)$ y exploraremos la contribución local a la atenuación en el contexto del modelo de White.

\subsubsection{Análisis de la atenuación sísmica}

Con la intención de comparar las definiciones (3.1), (3.2), y (3.3) en el contexto del modelo de White, consideramos una matriz homogénea con las propiedades de la arenisca Utsira (Rubino et al., 2011), saturada con $\mathrm{CO}_{2}$ y agua. La saturación global de $\mathrm{CO}_{2}$ de la muestra se determina por el cociente entre el espesor de la semicapa saturada con $\mathrm{CO}_{2}, L_{1}$, y el espesor total de la muestra $L=L_{1}+L_{2}=1 \mathrm{~m}$. Las propiedades físicas de la matriz y de los fluidos porales están expresadas en la Tabla 3.1.

Las Figuras 3.3a y 3.3b muestran el inverso del factor de calidad en función de la frecuencia $f=\omega / 2 \pi$ para dos casos, con saturaciones globales de $\mathrm{CO}_{2}$ de $10 \%$ y $90 \%$, respectivamente. La solución que emplea la hipótesis de medio viscoelástico equivalente $1 / Q_{p}^{\mathcal{D}}$ está representada por una línea roja sólida, mientras que las soluciones basadas en consideraciones energéticas, $1 / Q_{p}^{\mathcal{M}}$ y $1 / Q_{p}^{\mathcal{A}}$, están representadas por una línea sólida verde y una línea azul discontinua, respectivamente. Observamos que existe un acuerdo excelente entre $Q_{p}^{\mathcal{D}}$ y $Q_{p}^{\mathcal{A}}$ para ambos valores de saturación de $\mathrm{CO}_{2}$, lo cual sostiene la relación establecida por O'Connell y Budiansky (1978) entre las ecuaciones (3.1) y (3.3), derivadas para modelos viscoelásticos simples, incluso cuando el medio viscoelástico es la contraparte equivalente de un medio poroelástico. Para el caso de baja saturación de $\mathrm{CO}_{2}$, tenemos que $Q_{p}^{\mathcal{M}} \lesssim 10$ en la vecindad del máximo de atenuación (Figura 3.3a) y, por lo tanto, nos encontramos por fuera del rango donde la aproximación $\mathcal{W}_{\max } \simeq 2 \overline{\mathcal{W}}$ es válida. Consecuentemente, el factor de calidad $Q_{p}^{\mathcal{M}}$ tiende a discrepar 
de $Q_{p}^{\mathcal{D}}$ y $Q_{p}^{\mathcal{A}}$, con una diferencia relativa de $\sim 10 \%$. Sin embargo, este no es el caso en la Figura $3.3 \mathrm{~b}$, ya que aquí los valores de $Q_{p}^{\mathcal{M}}$ se encuentran dentro del rango donde la aproximación es válida. Estos comportamientos son similares a aquellos esperados para sólidos viscoelásticos y verifican que la hipótesis de medio viscoelástico equivalente está en acuerdo con las definiciones basadas en consideraciones energéticas para el modelo de White.

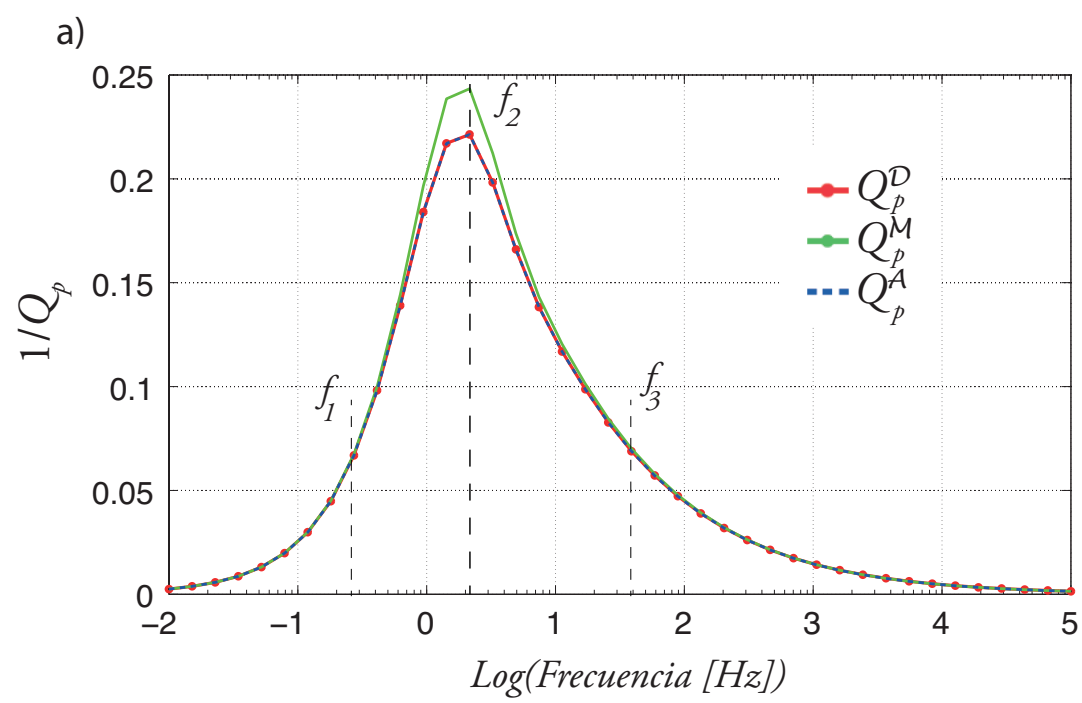

b)

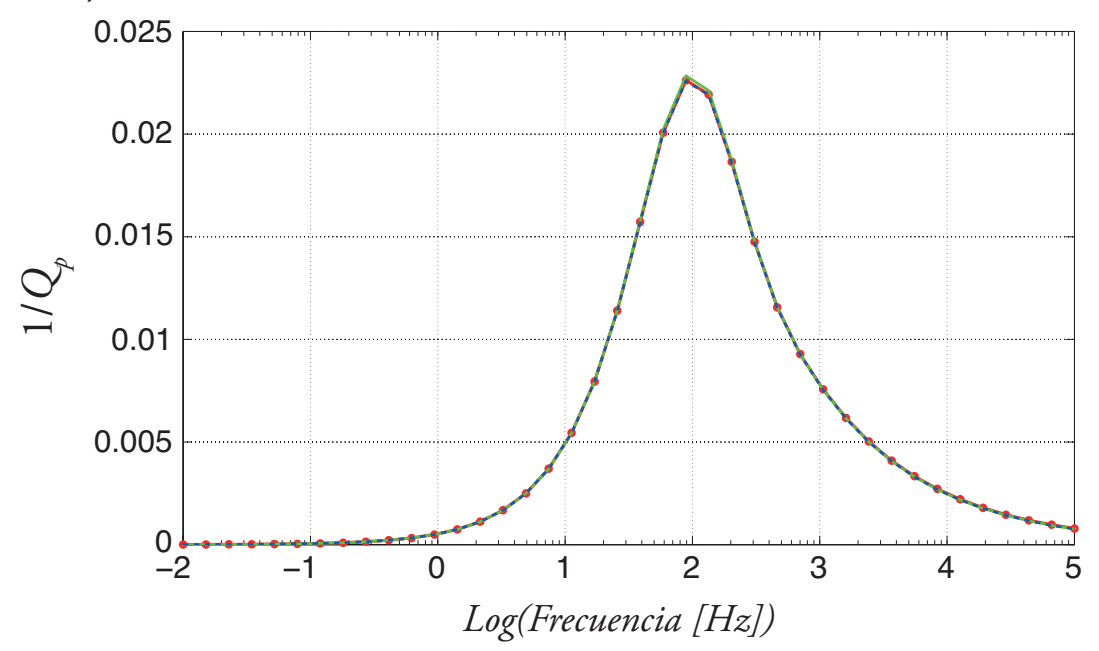

Figura 3.3. Inverso del factor de calidad en función de la frecuencia para una roca compuesta por una arena Utsira saturada de manera alternada por $\mathrm{CO}_{2}$ y agua. La saturación global de $\mathrm{CO}_{2}$ es (a) $10 \%$ y (b) $90 \%$. Las curvas corresponden a las tres metodologías presentadas en las ecuaciones (3.1), (3.2) y (3.3).

Las definiciones basadas en consideraciones energéticas poroelásticas nos permiten analizar el proceso de flujo inducido por onda en términos de las contribuciones locales $q^{-1}(y, f)$. La Figura 3.4 nos muestra la distribución espacial de $q^{-1}(y, f)$ para tres frecuencias, indicadas en la Figura $3.3(\mathrm{a})$, es decir, $f_{1}=0.27 \mathrm{~Hz}, f_{2}=2.15 \mathrm{~Hz}, \mathrm{y}$ $f_{3}=38 \mathrm{~Hz}$. La frecuencia $f_{2}$ es aquella en la cual la atenuación es máxima, mientras que $f_{1}$ y $f_{3}$ se eligen de manera tal que $Q_{p}\left(f_{1}\right) \simeq Q_{p}\left(f_{3}\right)$. Es importante notar que si 


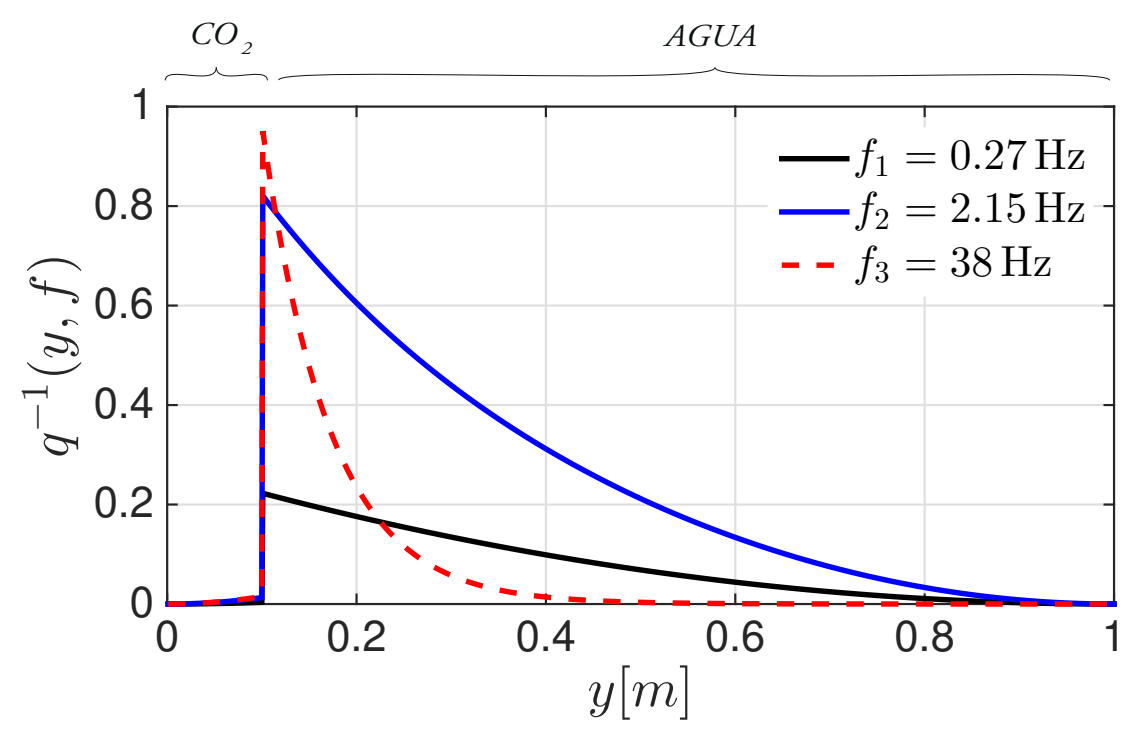

Figura 3.4. Contribución local a la atenuación en función de la distancia para el modelo de capas planas paralelas saturadas de forma alternada con agua y $\mathrm{CO}_{2}$. Se grafica la contribución para tres frecuencias, resaltadas en la Figura 3.3(a).

integramos $q^{-1}(y, f)$ a lo largo de cada curva de la Figura 3.4, obtendremos la atenuación global para esa frecuencia, graficada en la Figura 3.3(a). Una particularidad de la Figura 3.4 es que, independientemente de la frecuencia, las contribuciones locales a la atenuación global tienen lugar en la capa saturada con agua. Este comportamiento es esperado, ya que la disipación viscosa tiene lugar, mayormente, en las zonas saturadas con el fluido de mayor viscosidad y cerca de la interfase $\mathrm{CO}_{2}$-agua, donde el gradiente de presión del fluido poral es máximo.

Los cambios en la distribución espacial de $q^{-1}(y, f)$ con la frecuencia pueden analizarse observando el comportamiento de las curvas de la Figura 3.4. Para frecuencias menores a la frecuencia asociada al máximo de atenuación, es decir, por ejemplo, para $f_{1}$, la contribución local (curva negra) posee un máximo en la discontinuidad agua$\mathrm{CO}_{2}$, y luego disminuye lentamente con la distancia a la interfase. Este comportamiento cambia al considerar la frecuencia asociada al máximo de atenuación $f_{2}$. En este caso observamos nuevamente un máximo en la curva de $q^{-1}\left(y, f_{2}\right)$ (curva azul) en la interfase agua- $\mathrm{CO}_{2}$, mayor al de $q^{-1}\left(y, f_{1}\right)$. Sin embargo, la contribución local disminuye de forma más abrupta con la distancia. A su vez, el área bajo la curva es significativamente mayor, lo cual es esperable ya que $f_{2}$ es la frecuencia asociada al máximo de atenuación. Para frecuencias mayores al máximo, como es el caso de $f_{3}$, la concavidad de la curva $q^{-1}\left(y, f_{3}\right)$ (curva roja) se acentúa, evidenciando una contribución alta en las regiones cercanas a la discontinuidad, mayor a las de $q^{-1}\left(y, f_{1}\right)$ y $q^{-1}\left(y, f_{2}\right)$, que se vuelve prácticamente nula para regiones alejadas. Este comportamiento puede ser analizado empleando la definición de la longitud de difusión $L_{d}(\omega)$ (ecuación (2.50)). Como $L_{d}$ es inversamente proporcional a la frecuencia, la extensión espacial de las regiones que contribuyen a disipar la energía tiende a acortarse cuanto mayor es la frecuencia de la onda, haciendo que las curvas de $q^{-1}(y, f)$ aumenten su concavidad. Una característica que es posible analizar a través de la contribución local a la atenuación es que, a pesar de que las curvas $q^{-1}\left(y, f_{1}\right)$ y $q^{-1}\left(y, f_{3}\right)$ son significativamente diferentes, el área bajo 
ambas es idéntica, por lo cual la atenuación es igual en ambos casos.

Evidentemente, el modelo de White considera una distribución de fluidos simple, lo cual permite estudiar de manera directa la dependencia de cada parámetro físico en el comportamiento de la atenuación por flujo inducido por onda en la mesoescala. A continuación consideraremos el caso de distribuciones estocásticas de fluidos. En este contexto, analizaremos la atenuación en función de la frecuencia y, luego, estudiaremos la contribución local a la atenuación.

\subsection{Modelo estocástico 2-D de saturación «patchy»}

Estudiaremos ahora la atenuación por flujo inducido por onda en la mesoescala en medios 2-D parcialmente saturados. Para ello, tomamos una roca homogénea de $1 \mathrm{~m}$ de lado saturada con una distribución espacial heterogénea de dos fluidos inmiscibles, con compresibilidades diferentes. Las propiedades físicas de la matriz (arenisca Utsira) y fluidos porales (agua y $\mathrm{CO}_{2}$ ) son idénticas a las propiedades consideradas en el caso del modelo de White analizado en la Sección anterior (Tabla 3.1). En este caso, resolvemos las ecuaciones de consolidación de Biot (1941) considerando un experimento oscilatorio de relajación, utilizando las condiciones de borde descriptas por las ecuaciones (3.8)(3.11). El sistema de ecuaciones resultante es resuelto por medio de un método de elementos finitos que permite encontrar los campos de desplazamiento $\hat{\mathbf{u}} \mathrm{y}$ w en función del espacio y la frecuencia (Rubino et al., 2009, 2013b). Este método emplea funciones bilineales para aproximar los vectores de desplazamiento del sólido y un subespacio cerrado de la parte vectorial del espacio de Raviart-Thomas-Nedelec de orden cero para representar el desplazamiento relativo del fluido (Raviart y Thomas, 1977; Nedelec, 1980).

Una gran cantidad de propiedades petrofísicas asociadas a depósitos sedimentarios exhiben características de escala invariante o fractales y, por ello, pueden ser modeladas satisfactoriamente empleando funciones de covarianza de tipo von Kármán (p. ej., Tronicke y Holliger, 2005). Para generar una distribución binaria altamente irregular de fluidos utilizaremos una función de densidad espectral del tipo von-Kármán que responde a (Rubino y Holliger, 2012)

$$
S_{d}\left(k_{x}, k_{y}\right)=S_{0}\left(1+k_{x} a_{x}^{2}+k_{y}^{2} a_{y}^{2}\right)^{-(H+E / 2)},
$$

donde $k_{x}$ y $k_{y}$ son los números de onda vertical y horizontal, $a_{x} \mathrm{y} a_{y}$ son las longitudes de correlación horizontal y vertical, $S_{0}$ es una constante de normalización, y $E$ es la dimensión euclídea. El parámetro $H$ varia entre 0 y 1 y determina la complejidad del proceso estocástico. Esta expresión corresponde a un proceso estocástico de banda limitada y escala invariante con una dimensión fractal de Hausdorff $D_{H}=E+1-H$. Para obtener la distribución de fluidos a partir de la ecuación (3.62), asignamos a cada celda $\Omega_{j k}$ del dominio computacional un número pseudoaleatorio a partir de una distribución uniforme. Luego, tomamos la transformada de Fourier de este campo al dominio del número de onda y filtramos su espectro de amplitud utilizando la ecuación (3.62). Luego, transformamos el resultado nuevamente al dominio espacial y obtenemos una distribución aleatoria de números en la forma de una matriz de $N_{x} \times N_{y}$ valores. Luego, utilizamos una transformación lineal para mapear estos valores al intervalo 
$[0,1]$. Finalmente, el campo de saturación binario de interés se obtiene por medio de «thresholding». Es decir, se compara numéricamente el valor obtenido en cada elemento de esta matriz con un valor umbral $^{2}$ determinado. Luego, toda celda cuyo valor sea mayor a al valor umbral se considera completamente saturada con agua, de modo contrario, se considera completamente saturada con gas.

La Figura 3.5 muestra la distribución obtenida empleando este procedimiento numérico, la cual corresponde a una longitud isotrópica de correlación de $a_{y}=a_{x}=36 \mathrm{~cm}$, $H=0.8$ y un valor umbral de 0.41 para realizar la binarización. Se observa una distribución de fluidos donde $\mathrm{CO}_{2}$ (regiones negras) y agua (regiones blancas) saturan a una arena homogénea con una saturación global de $\mathrm{CO}_{2}$ de $20 \%$.

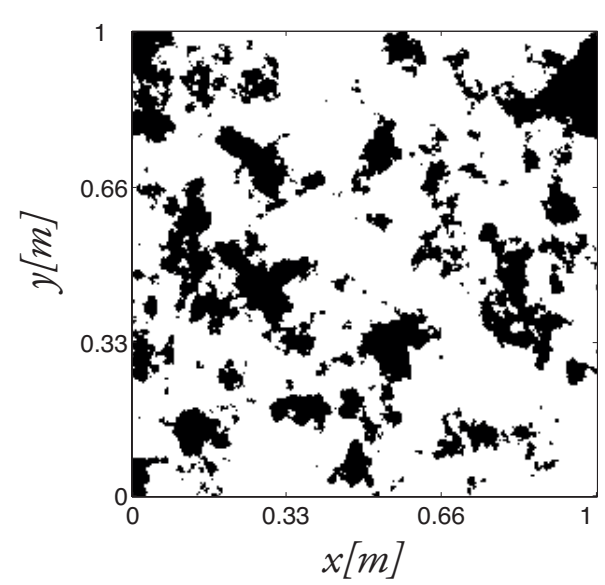

Figura 3.5. Campo de saturación parcial binaria de $\mathrm{CO}_{2}$ (regiones negras) y agua (regiones blancas) considerado para calcular la atenuación sísmica. La saturación global de $\mathrm{CO}_{2}$ es del $20 \%$. Notar que la matriz de roca es homogénea y sus propiedades se corresponden con las de la arenisca Utsira (Tabla 3.1).

Es importante recalcar que, a pesar de su simplicidad, los modelos de rocas homogéneas que contienen «patches» binarios han sido considerados en el pasado por varios autores para modelar la respuesta sísmica de ambientes parcialmente saturados (p. ej., White, 1975; Dutta y Odé, 1979a; Johnson, 2001; Norris, 1993; Rubino et al., 2009; Masson y Pride, 2011). Como hemos resaltado previamente, este tipo de patrones de saturación no respeta las leyes de flujo en medios porosos. Sin embargo, la elección de este tipo de modelo binario permite explorar las características del proceso de difusión evitando las complejidades asociadas a distribuciones de fluidos realistas con características continuas. En este sentido, abordaremos el estudio de modelos con campos de saturación realistas, asociados a leyes de flujo en medios porosos, en los Capítulos 5 y 6 de esta Tesis.

\footnotetext{
${ }^{2}$ Del inglés: «Threshold value».
} 


\subsubsection{Análisis de la atenuación sísmica}

La Figura 3.6 muestra el inverso del factor de calidad en función de la frecuencia para las diferentes definiciones propuestas en las ecuaciones (3.1), (3.2) y (3.3). Observamos que la curva de atenuación asociada a la hipótesis de medio viscoelástico equivalente $Q_{p}^{\mathcal{D}}$ (línea roja) posee un buen acuerdo con la curva que se obtiene utilizando la metodología basada en el cálculo de la energía elástica promedio $Q_{p}^{\mathcal{A}}$ (línea azul punteada). Sin embargo, los valores del factor de calidad en las cercanías del máximo de atenuación caen por fuera del rango donde $Q_{p}^{\mathcal{M}} \gtrsim 10$, es decir, por fuera del rango de validez de la aproximación $\mathcal{W}_{\max } \simeq 2 \overline{\mathcal{W}}$. Observamos que, en esta situación, hay discrepancias entre la metodología basada en el cálculo de la energía elástica máxima $Q_{p}^{\mathcal{M}}$ (línea verde) y las otras estimaciones del factor de calidad, con una diferencia relativa máxima de $\sim 5 \%$. Estos resultados, en conjunto con los resultados obtenidos para el modelo de White, indican que las estimaciones basadas en la hipótesis de medio viscoelástico equivalente poseen un muy buen acuerdo con aquellas derivadas de la ecuación (3.3). Además, observamos que las diferencias de $Q_{p}^{\mathcal{D}}$ y $Q_{p}^{\mathcal{A}}$ respecto de la definición clásica $Q_{p}^{\mathcal{M}}$ son significativas solo en presencia de niveles de atenuación muy altos. Estos comportamientos son similares a aquellos esperados para sólidos viscoelásticos, y por ello, verifican que la hipótesis de medio viscoelástico equivalente se condice con los resultados obtenidos utilizando definiciones energéticas.

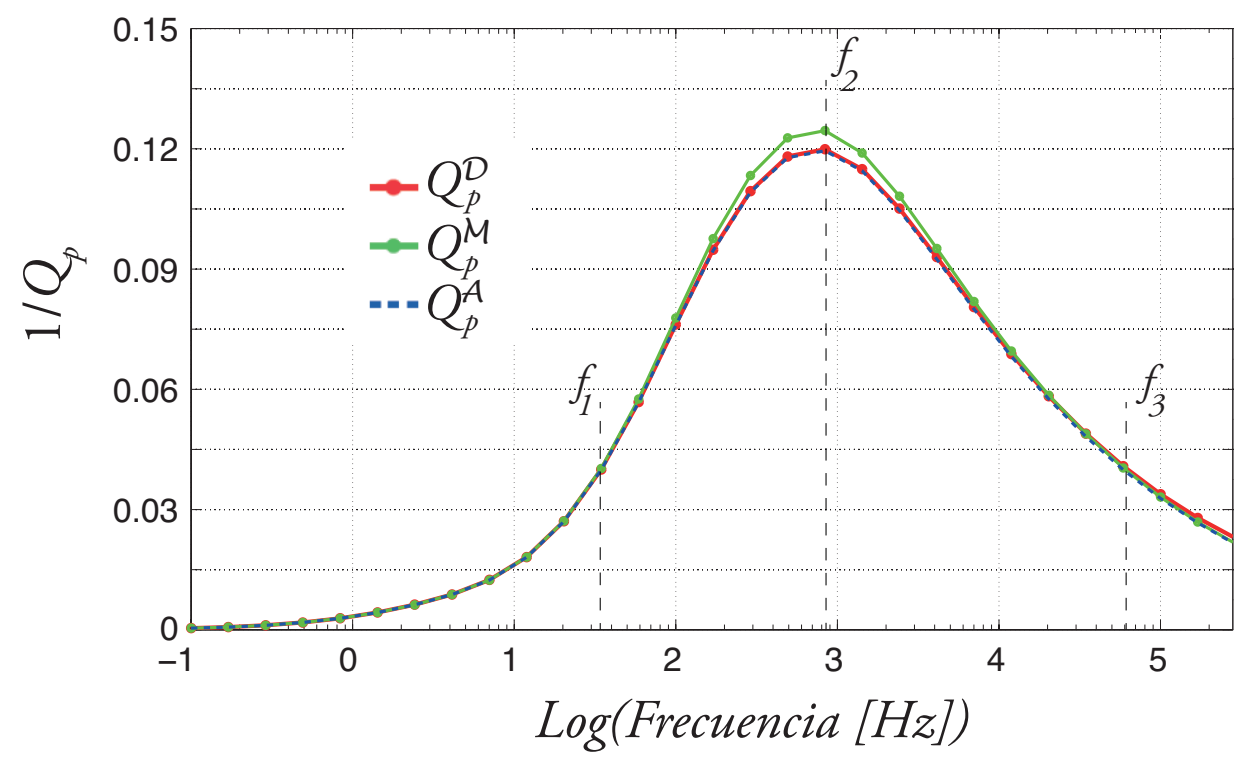

Figura 3.6. Inverso del factor de calidad en función de la frecuencia para una roca homogénea saturada con la distribución de fluidos porales que muestra la Figura 3.5. Las diferentes curvas corresponden a las tres definiciones de atenuación presentadas a través de las ecuaciones (3.1), (3.2), y (3.3).

Las definiciones basadas en consideraciones energéticas poroelásticas nos permiten analizar el proceso de flujo inducido por onda en términos de las contribuciones locales $q_{k l}^{-1}(\omega)$ (ecuación (3.28)). La Figura 3.7 nos muestra la distribución espacial de $q_{k l}^{-1}$ para las frecuencias $f_{1}=34.5 \mathrm{~Hz}, f_{2}=837.7 \mathrm{~Hz}$, y $f_{3}=5.8 \times 10^{4} \mathrm{~Hz}$ (indicadas en la Figura 3.6). En este contexto, $f_{2}$ es la frecuencia en la cual la atenuación es máxima. 
Por otro lado, $f_{1}$ y $f_{3}$ se eligen de manera tal que $Q_{p}\left(f_{1}\right)=Q_{p}\left(f_{3}\right)$. Es importante notar que si integramos los valores de $q_{k l}^{-1}$ en cada panel de la Figura 3.7, obtendremos la atenuación global para esa frecuencia, graficada en la Figura 3.6. Al igual que en el caso del modelo de White, observamos en la Figura 3.7 que, independientemente de la frecuencia, las contribuciones locales a la atenuación global tienen lugar en las zonas saturadas con agua que rodean a los «patches» de $\mathrm{CO}_{2}$. Este comportamiento es esperado, ya que, como fue observado en la Figura 3.4, la disipación tiene lugar, mayormente, en las zonas saturadas con el fluido más viscoso y cerca de la interfase $\mathrm{CO}_{2}$-agua, donde el gradiente de presión del fluido poral es máximo.

Los cambios en la distribución espacial de $q_{k l}^{-1}(\omega)$ con la frecuencia pueden analizarse teniendo en cuenta la longitud de difusión $L_{d}(\omega)$ (ecuación (2.50)). Podemos observar que, para la frecuencia $f_{1}$, las regiones donde $q_{k l}^{-1}$ es significativo, son irregulares, y desaparecen completamente alrededor de algunos «patches» de $\mathrm{CO}_{2}$. Esto se debe a que, para esta frecuencia baja, la longitud de difusión excede la distancia que separa los «patches» de $\mathrm{CO}_{2}$ cercanos. Luego, estas regiones se comportan, de manera efectiva, como un «patch» de tamaño relativamente grande $\mathrm{y}$, en su interior, las contribuciones $q_{k l}^{-1}$ son esencialmente despreciables. Al realizar una comparación de las Figuras 3.5 y 3.7a podemos observar que, para esta frecuencia, las regiones que contribuyen de manera significativa a la atenuación global de la muestra están relacionadas con los «patches» de agua con tamaños característicos grandes en comparación con las demás regiones de la roca y que se encuentran relativamente libres de pequeños «patches» de $\mathrm{CO}_{2}$. Para las frecuencias $f_{2}$ y $f_{3}$, las contribuciones a la disipación $q_{k l}^{-1}$ pueden observarse alrededor de todos los «patches» de $\mathrm{CO}_{2}$. Sin embargo, las regiones caracterizadas por altas contribuciones $q_{k l}^{-1}$ son mayores para $f=f_{2}$ y, por lo tanto, la atenuación global es también mayor.
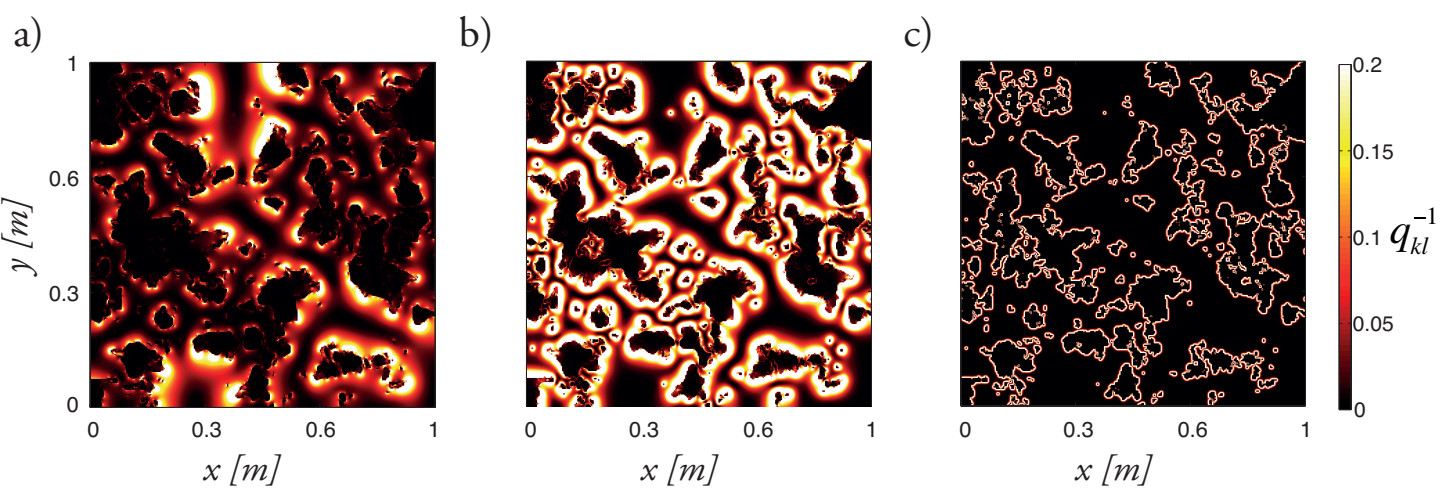

Figura 3.7. Distribución espacial de las contribuciones locales $q_{k l}^{-1}\left[\mathrm{~m}^{-2}\right]$ a la atenuación global para una roca homogénea que contiene la distribución de fluidos la muestra de roca de la Figura 3.5. Los paneles corresponden a tres frecuencias a) $\left.f_{1}=34.5 \mathrm{~Hz}, \mathrm{~b}\right) f_{2}=837.7 \mathrm{~Hz}$, y c) $f_{3}=5.8 \times 10^{4} \mathrm{~Hz}$, indicadas en la Figura 3.6.

Como la longitud de difusión es inversamente proporcional a la frecuencia (ecuación (2.50)), es esperable que la extensión espacial de las regiones con valores significativos de $q_{k}^{-1}$ se reduzca a las inmediaciones de los «patches» de $\mathrm{CO}_{2}$ al aumentar la frecuencia. Este comportamiento prevalece cuando las frecuencias se incrementan de $f_{2}$ a $f_{3}$ (Figuras $3.7 \mathrm{~b}$ y $3.7 \mathrm{c}$ ). Sin embargo, las Figuras 3.7 a y $3.7 \mathrm{~b}$ indican que, para 
las regiones saturadas con agua, la extensión espacial sobre la cual la energía se disipa se ensancha al aumentar la frecuencia de $f_{1}$ a $f_{2}$. Esto evidencia que existe una interacción entre «patches», es decir una interferencia entre procesos de flujo vecinos, que afecta el proceso de disipación. Además, es importante notar que, a pesar de que la distribución espacial y los valores locales de $q_{k l}^{-1}$ en las Figuras 3.7a y 3.7c son diferentes, la atenuación global para estas dos frecuencias es la misma (Figura 3.6). La ventaja de este análisis de atenuación local yace en poder identificar y analizar estas características, cuya interpretación permite comprender ciertos aspectos del procesos de disipación, tales como, por ejemplo, la interacción entre el procesos de relajación de presión en presencia de «patches» cercanos. Esto no es posible con métodos basados en la hipótesis de medio viscoelástico equivalente.

Hemos analizado la atenuación global y la contribución local $q_{k l}^{-1}$ en una roca homogénea parcialmente saturada con una distribución binaria de $\mathrm{CO}_{2}$ y agua. Es importante notar que la atenuación depende tanto de la disipación $\mathcal{P}$ como de la energía de deformación $\mathcal{W}$. En este sentido, las discrepancias observadas en la Figura 3.6 entre $Q_{p}^{\mathcal{M}}$ y $Q_{p}^{\mathcal{A}}$ se deben a que el máximo $\mathcal{W}_{\text {max }}$ y el doble de la media de la energía elástica de deformación $2 \overline{\mathcal{W}}$ difieren levemente en cierto rango de frecuencias. Consecuentemente, la energía elástica de deformación almacenada posee algún control en las características de las curvas de atenuación sísmica en función de la frecuencia. A continuación estudiaremos brevemente estas características.

\subsubsection{Análisis de la energía elástica de deformación}

Como lo sugieren las ecuaciones (3.2) y (3.3), la energía de deformación controla, al menos en parte, las características de las curvas de factor de calidad en función de la frecuencia. A continuación analizaremos sus características locales y globales. En particular, de acuerdo con la ecuación (2.18), la energía de deformación por unidad de volumen en cada celda computacional de material poroelástico se puede descomponer en

$$
\mathcal{W}_{k l}(t)=\mathcal{W}_{k l}^{s}(t)+\mathcal{W}_{k l}^{f}(t)
$$

donde

$$
\mathcal{W}_{k l}^{s}(t)=\frac{1}{2}(\sigma: \epsilon)_{k l}, \quad \mathcal{W}_{k l}^{f}(t)=\frac{1}{2}\left(p_{f} \zeta\right)_{k l}
$$

Los términos $\mathcal{W}_{k l}^{s}(t)$ y $\mathcal{W}_{k l}^{f}(t)$ denotan la contribución relacionada a la deformación macroscópica a contenido de fluido constante y la contribución relacionada con los cambios en el contenido de fluido a deformación macroscópica constante, respectivamente (Bourbié et al., 1987). Trabajos previos, donde se calcula la energía de deformación, no consideran la contribución $\mathcal{W}_{k l}^{f}(t)$ asociada al contenido de fluido (p. ej., Mavko y Nur, 1979; Müller y Rothert, 2006), por lo cual, resulta interesante explorar los efectos correspondientes sobre la energía de deformación de forma local y global.

Es importante resaltar que $\mathcal{W}_{k l}^{s}$ y $\mathcal{W}_{k l}^{f}$ no son energías «sensu stricto», y que, en determinadas situaciones, $\mathcal{W}_{k l}^{f}$ puede tomar valores negativos en el contexto de un experimento oscilatorio. Es decir, es posible que suceda que $\left(p_{f} \zeta\right)_{k l}<0$ en una celda 
determinada $\Omega_{k l}$. Este comportamiento puede tener lugar en dos escenarios diferentes:

$$
\begin{aligned}
& \text { 1) } p_{f}<0 \wedge \zeta>0, \quad \text { en } \Omega_{k l}, \\
& \text { 2) } p_{f}>0 \wedge \zeta<0, \quad \text { en } \Omega_{k l} .
\end{aligned}
$$

En el primer caso, la presión del fluido poral disminuye $p_{f}<0$ y el contenido de fluido aumenta $\zeta>0$. En este contexto, de acuerdo a la ecuación (2.17), la región debe estar sometida a una expansión macroscópica, es decir,

$$
\begin{aligned}
p_{f}=-\alpha M \nabla \cdot \mathbf{u}+M \zeta & <0, \\
\zeta & <\alpha \nabla \cdot \mathbf{u}, \\
0 & <\nabla \cdot \mathbf{u},
\end{aligned}
$$

donde hemos considerado que $\alpha>0$ y $M>0$ (p. ej., Biot y Willis, 1957). Luego, el primer escenario consiste en una disminución de presión $p_{f}<0$ causada por una expansión macroscópica de la región $\nabla \cdot \mathbf{u}>0$, en la cual, la porción del medio poroelástico comprendido en el elemento $\Omega_{k l}$ «succiona» el fluido de los elementos vecinos, aumentando el contenido de fluido $\zeta>0$.

En el segundo caso, la presión del fluido poral aumenta $p_{f}>0$ y el contenido de fluido disminuye $\zeta<0$. En este contexto, de acuerdo a la ecuación (2.17), $\nabla \cdot \mathbf{u}<0$. Por lo cual, el segundo escenario consiste en un aumento de presión debido a una compresión macroscópica, donde, el elemento $\Omega_{k l}$ «expulsa» el fluido a las regiones vecinas.

De acuerdo a la ecuación (3.24), para una frecuencia $\omega$ dada, la energía elástica de deformación promedio en cada celda computacional de material poroelástico, en función de la transformada de Fourier de los desplazamientos, se puede expresar como

$$
\overline{\mathcal{W}_{k l}}(\omega)=\overline{\mathcal{W}_{k l}^{s}}(\omega)+\overline{\mathcal{W}_{k l}^{f}}(\omega)
$$

donde

$$
\overline{\mathcal{W}_{k l}^{s}}(\omega)=\frac{\delta^{2}}{4} \Re\left(\hat{\sigma}: \hat{\epsilon}^{*}\right)_{k l}, \quad \overline{\mathcal{W}_{k l}^{f}}(\omega)=\frac{\delta^{2}}{4} \Re\left(\hat{p}_{f} \hat{\zeta}^{*}\right)_{k l}
$$

Empleando esta definición, podemos calcular la distribución espacial de la energía elástica de deformación promedio en respuesta al desplazamiento generado por una onda $\mathrm{P}$ que viaja a través de una muestra homogénea de arenisca Utsira saturada por la distribución de fluidos graficada en la Figura 3.5. Este comportamiento se muestra en la Figura 3.8 para tres frecuencias de oscilación: una frecuencia muy baja $\left(f_{\text {low }}=\right.$ $0.1 \mathrm{~Hz}$ ) asociada a un estado relajado (columna izquierda), la frecuencia asociada al máximo de atenuación $\left(f_{\max }=837.7 \mathrm{~Hz}\right)$ (columna del medio) y una frecuencia muy alta $\left(f_{\text {high }}=10^{5} \mathrm{~Hz}\right)$ asociada a un estado poco relajado (columna derecha). La fila superior corresponde a la distribución espacial de la energía de deformación total $\overline{\mathcal{W}_{k l}}$, la fila central corresponde a la contribución $\overline{\mathcal{W}_{k l}^{s}}$ y la inferior a $\overline{\mathcal{W}_{k l}^{f}}$.

Comparando la Figura 3.8a con la Figura 3.5, podemos observar que para frecuencias mucho menores a la frecuencia máxima $f_{\max }$ las regiones caracterizadas por valores relativamente altos de energía promedio de deformación corresponden a los «patches» de $\mathrm{CO}_{2}$. Además, para este régimen de bajas frecuencias $\overline{\mathcal{W}_{k l}^{s}}$ posee una distribución relativamente homogénea (Figura 3.8d) y, por lo tanto, las irregularidades observadas en 

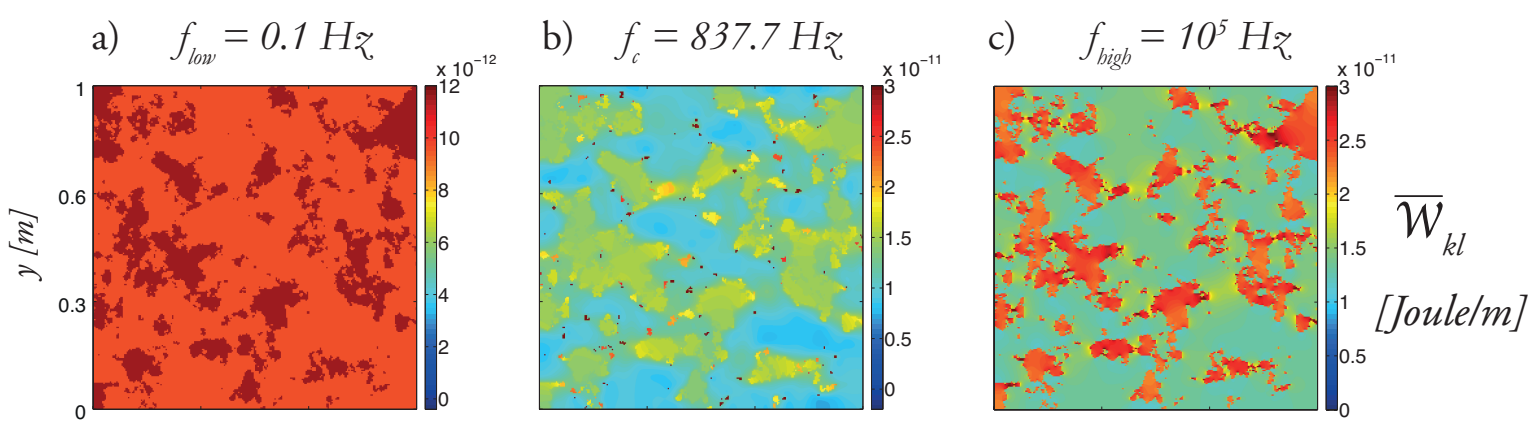

d)

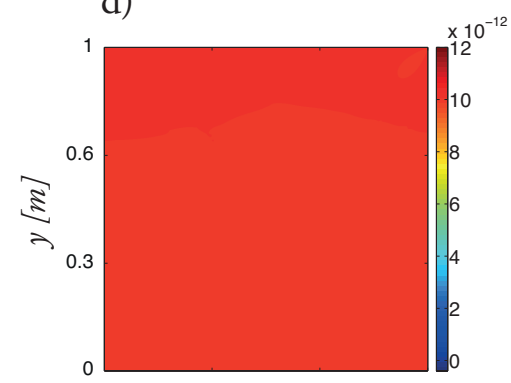

e)

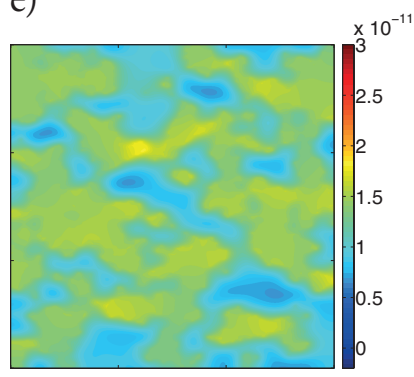

f)

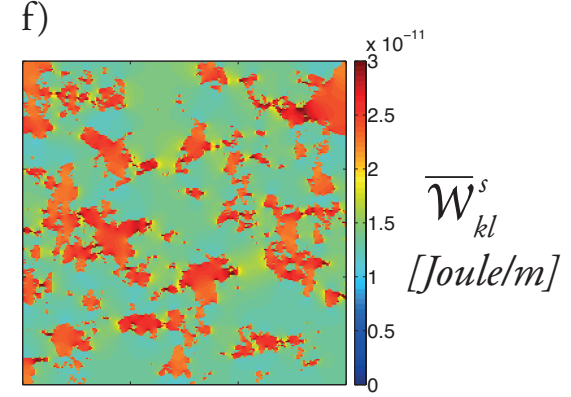

g)

h)
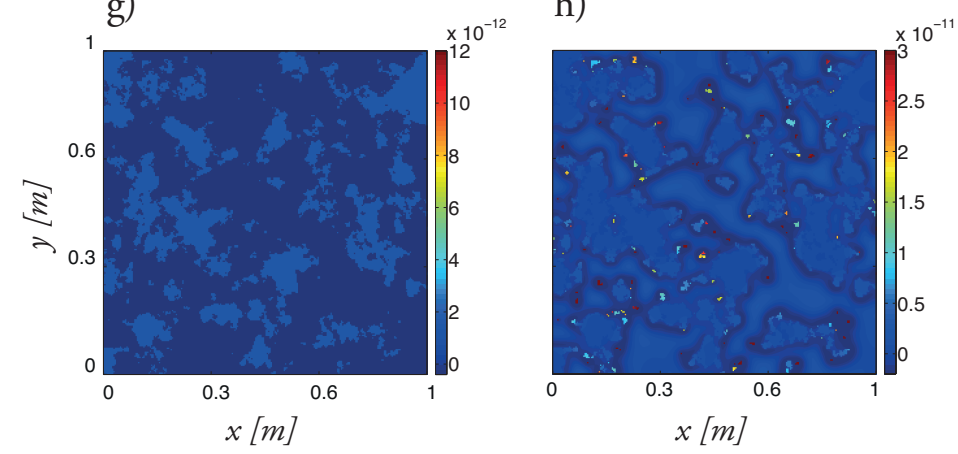

i)

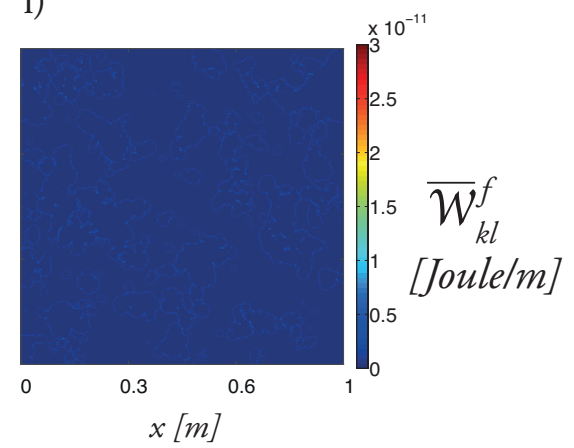

Figura 3.8. Energía elástica de deformación promedio correspondiente a una roca homogénea saturada con la distribución de fluidos graficada en la Figura 3.5. Las filas corresponden a la distribución espacial de la energía de deformación promedio total $\overline{\mathcal{W}_{k l}}$ (fila superior), a las componentes $\overline{\mathcal{W}_{k l}^{s}}$ (fila del medio) y $\overline{\mathcal{W}_{k l}^{f}}$ (fila inferior). Las columnas corresponden una frecuencia relativamente baja $f=0.1 \mathrm{~Hz}$ (columna izquierda), a la frecuencia del máximo de atenuación (columna del medio) y a una frecuencia relativamente alta $f=10^{5} \mathrm{~Hz}$ (columna derecha). 
la Figura 3.8a están controladas por la contribución asociada a cambios en el contenido de fluidos (Fig. 3.8g).

Para la frecuencia $f_{\max }$ asociada al máximo de atenuación, los valores máximos de energía de deformación promedio tienen lugar en los «patches» de $\mathrm{CO}_{2}$ (Figura 3.8b). En este caso, la energía de deformación promedio se encuentra controlada principalmente por las deformaciones a contenido de fluido constante $\overline{\mathcal{W}_{k l}^{s}}$ (Figura 3.8e). La contribución $\overline{\mathcal{W}_{k l}^{f}}$ muestra algunos valores negativos alrededor de los «patches» de $\mathrm{CO}_{2}$ (Figura 3.8h). Sin embargo, hemos verificado que estos los valores $\overline{\mathcal{W}^{f}}$ son despreciables frente a los valores tomados por $\overline{\mathcal{W}^{s}}$ en este caso.

Finalmente, para una frecuencia $f_{\text {high }}$, mucho mayor a $f_{\text {max }}$, observamos que las regiones que contienen los valores más altos de energía de deformación promedio nuevamente coinciden con los «patches» de $\mathrm{CO}_{2}$ (Figura 3.8c). Esto es esperable, ya que nos encontramos en un estado poco relajado y no hay tiempo suficiente en un semiperíodo para que haya un considerable intercambio de presión entre los «patches» y su entorno. La energía de deformación es entonces controlada por el término asociado a deformación a contenido de fluido constante (Figura 3.8f).

La Figura 3.9 muestra la contribución $\overline{\mathcal{W}^{s}}$ (curva azul) y la energía de deformación promedio total $\overline{\mathcal{W}}$ (círculos rojos) para toda la muestra. Se observa que $\overline{\mathcal{W}} \simeq \overline{\mathcal{W}^{s}}$. Esto indica que, a pesar de que la contribución de la energía de deformación promedio relacionada a cambios en el contenido de fluido $\overline{\mathcal{W}^{f}}$ puede ser observada y analizada (Figura 3.8), su efecto a escala de REV es despreciable frente al efecto de $\overline{\mathcal{W}^{s}}$. Además, en la Figura 3.9 se grafica la energía de deformación máxima $\mathcal{W}_{\text {max }}$ para la muestra (curva verde). Observamos que en los entornos de la frecuencia crítica $f_{\max }, \mathcal{W}_{\max }$ es menor que $\overline{\mathcal{W}}$, lo cual explica por qué $1 / Q_{p}^{\mathcal{M}}$ es mayor que $1 / Q_{p}^{\mathcal{A}}$ (Figura 3.6).

Es importante notar que tanto el modelo de roca analizado en esta Sección, como el modelo 1-D de White analizado previamente, consisten en una matriz homogénea parcialmente saturada. En este sentido, los contrastes de compresibilidad se encuentran asociados a las propiedades de los fluidos saturantes. Como hemos anticipado, nuestro objetivo es estudiar la atenuación por flujo inducido por onda en rocas heterogéneas saturadas por fluidos inmiscibles, de manera de mejorar los modelos de saturación parcial usualmente empleados. Para ello, es importante explorar la atenuación global y la contribución local a la atenuación en escenarios donde la matriz es heterogénea. Un caso interesante para explorar es el de medio fracturado. A diferencia de los medios saturados por «patches», que solo generan atenuación en ondas compresionales, los medios fracturados generan atenuación tanto para las ondas compresionales como para las ondas de corte. En la próxima Sección validaremos la hipótesis de medio viscoelástico equivalente para ondas $\mathrm{P}$ y $\mathrm{S}$ en un medio fracturado saturado por un único fluido. A su vez, analizaremos la atenuación global y las correspondientes contribuciones locales para ambos tipos de onda.

\subsection{Modelo 2-D de medio fracturado}

La presencia de fracturas puede producir altos niveles de atenuación sísmica por flujo inducido por onda. Esto se debe al considerable contraste de compresibilidad que existe entre estos objetos y el medio poroso circundante (e.g. Rubino et al., 2014). A conti- 


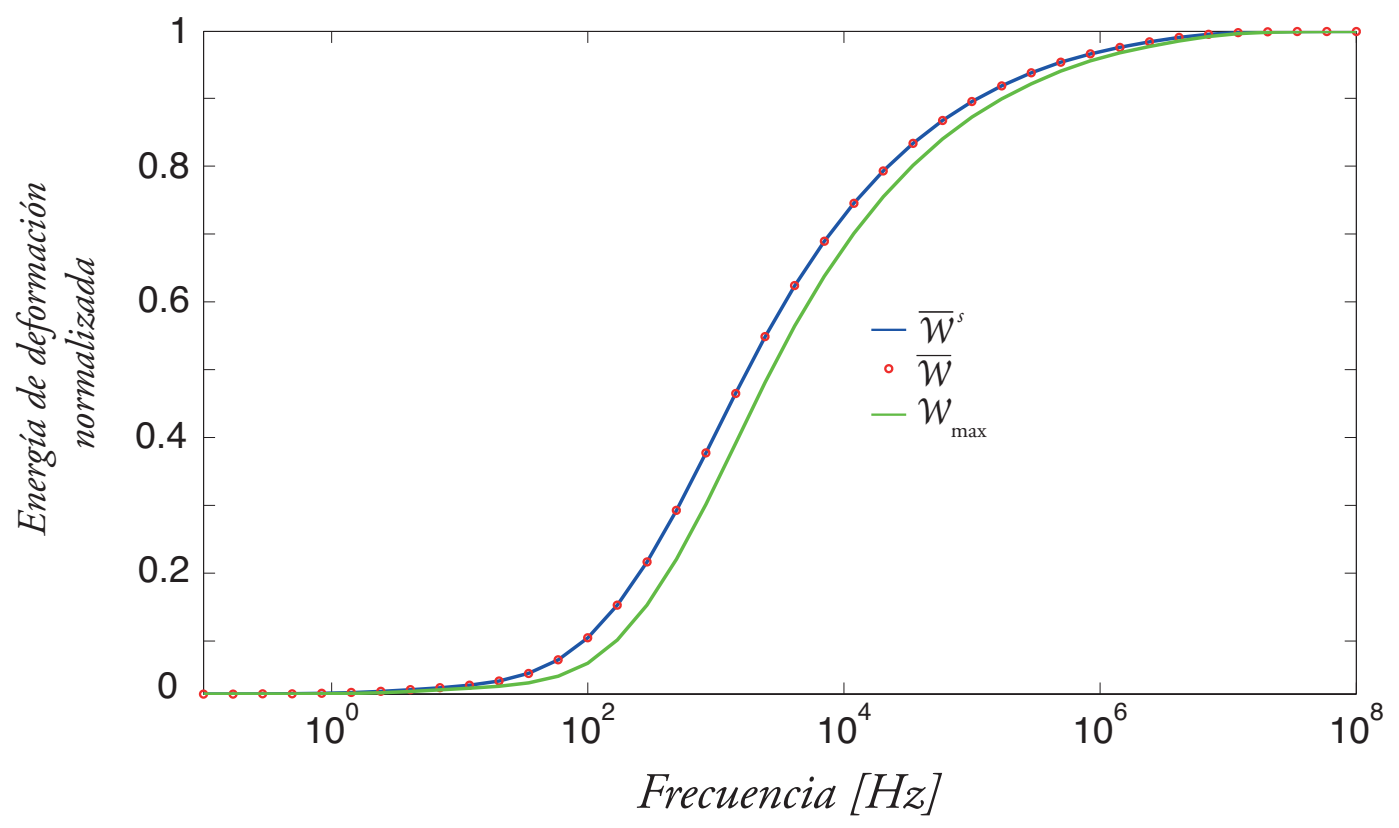

Figura 3.9. Energía elástica de deformación en el REV normalizada respecto del máximo en función de la frecuencia para la muestra de roca analizada. Las curvas representan la energía de deformación promedio total $\overline{\mathcal{W}}$ (círculos rojos), la contribución $\overline{\mathcal{W}^{s}}$ (curva azul), y $\mathcal{W}_{\max }$ (curva verde)

nuación analizaremos la relación entre las definiciones (3.1), (3.2), (3.3) en un medio fracturado, para ondas P y S viajando en la dirección vertical. Utilizaremos los experimentos oscilatorios descriptos en la Sección 3.2 de este trabajo de Tesis. La muestra de roca representativa está ilustrada en la Figura 3.10. Esta consiste de una roca porosa de $2.75 \mathrm{~cm}$ de lado que contiene una fractura elíptica inclinada $45^{\circ}$ respecto de la horizontal. El tamaño de la muestra de roca y las dimensiones de la fractura consideradas están en acuerdo con las escalas usualmente empleadas al estudiar los efectos del flujo inducido en medios fracturados (p. ej., Rubino et al., 2014; Milani et al., 2016; Barbosa et al., 2017).

En general, los modelos poroelásticos suelen considerar a las fracturas como parte del medio poroso (Brajanovsky et al., 2005; Nakagawa y Schoenberg, 2007; Gurevich et al., 2009; Rubino et al., 2013a, 2014). Como fue resaltado por Rubino et al. (2014), la representación efectiva de una fractura como parte de un medio poroelástico es consistente con el hecho de que las fracturas tienden a estar caracterizadas por superficies corrugadas, lo cual implica que las paredes de la fractura están en contacto entre sí en ciertas regiones. Además, las regiones entre estas áreas de contacto contienen fragmentos de rocas, productos del «weathering», o depósitos minerales. Consecuentemente, modelamos la fractura como un medio con una muy alta porosidad y permeabilidad, alojado en una arenisca relativamente dura cuyas propiedades están dadas en la Tabla 3.1 (Arenisca). A su vez, consideraremos que ambos medios poseen las mismas propiedades a nivel de grano. Tomamos una apertura máxima $h$ de $0.033 \mathrm{~cm}$ y, siguiendo el trabajo de Nakagawa y Schoenberg (2007), utilizamos $K_{m}^{f}=0.02 \mathrm{GPa}$ y $\mu_{m}^{f}=0.01 \mathrm{GPa}$ para representar las propiedades de la matriz seca que representa la fractura (Rubino et al., 2014). Para el material que rellena la fractura asumimos una porosidad $\phi^{f}$ de 


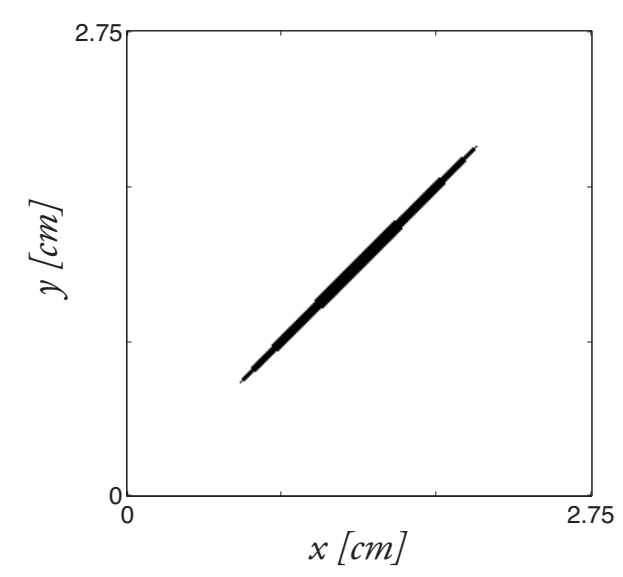

Figura 3.10. Muestra de roca que contiene una fractura elíptica inclinada a $45^{\circ}$, con una apertura máxima $h$ de $0.033 \mathrm{~cm}$. La región de matriz no fracturada (región blanca) corresponde a una arenisca de baja porosidad y la fractura (región negra) se encuentra modelada como una región de alta porosidad y permeabilidad. La muestra se encuentra completamente saturada con agua.

0.5 y una permeabilidad $\kappa^{f}$ de $100 \mathrm{D}$. Por último, supondremos que tanto la fractura como la matriz de roca se encuentran completamente saturadas por agua (Tabla 3.1).

\subsubsection{Análisis de la atenuación sísmica}

La Figura 3.11 muestra una comparación del inverso del factor de calidad para las ondas P y S utilizando las definiciones de atenuación dadas según las ecuaciones (3.1), (3.2), y (3.3). En el caso de la atenuación de onda P (Figura 3.11a), las tres curvas son muy similares a lo largo del ancho de banda graficado. Por otro lado, en el caso de la atenuación de onda $\mathrm{S}$ (Figura 3.11b) observamos que $Q_{s}^{\mathcal{M}}$ se desvía levemente respecto de $Q_{s}^{\mathcal{D}}$ y $Q_{s}^{\mathcal{A}}$ cuando $Q_{s}^{\mathcal{M}} \lesssim 10$. Estos resultados, cuyas características son similares a los esperados para medios viscoelásticos lineales, evidencian que la hipótesis de medio viscoelástico equivalente se encuentra en acuerdo con las estimaciones basadas en consideraciones energéticas también en casos que presentan considerables contrastes de propiedades mecánicas de la matriz.

Exploremos entonces las contribuciones locales a la atenuación $q_{k l}^{-1}$ para ambas ondas. Las Figuras 3.12 y 3.13 muestran las contribuciones locales para las frecuencias indicadas en la Figura 3.11: $f_{1}=0.2 \mathrm{~Hz}$, la frecuencia del máximo de atenuación $f_{2}=2.15 \mathrm{~Hz}$, y $f_{3}=74.44 \mathrm{~Hz}$. Observamos que tanto para las ondas $\mathrm{S}$ como para las ondas $\mathrm{P}$ la disipación tiene lugar principalmente en la matriz de roca cercana a la fractura. Esto es esperable, ya que la disipación de la energía es inversamente proporcional a la permeabilidad (ecuación (3.21)). Por lo tanto, la disipación se produce en mayor medida en las regiones de baja permeabilidad afectadas por el flujo inducido por onda, es decir, en las inmediaciones de las fracturas. Es interesante notar que, mientras que la contribución local muestra un patrón «en forma de z» para las ondas P (Figura $3.12 \mathrm{~b}$ ), el correspondiente patrón para las ondas $\mathrm{S}$ es relativamente simétrico respecto a la fractura (Figura 3.13b). Podemos notar también que, tanto para las ondas S como para las $\mathrm{P}$, la región que contribuye mayormente a la atenuación global se encuentra 
a)

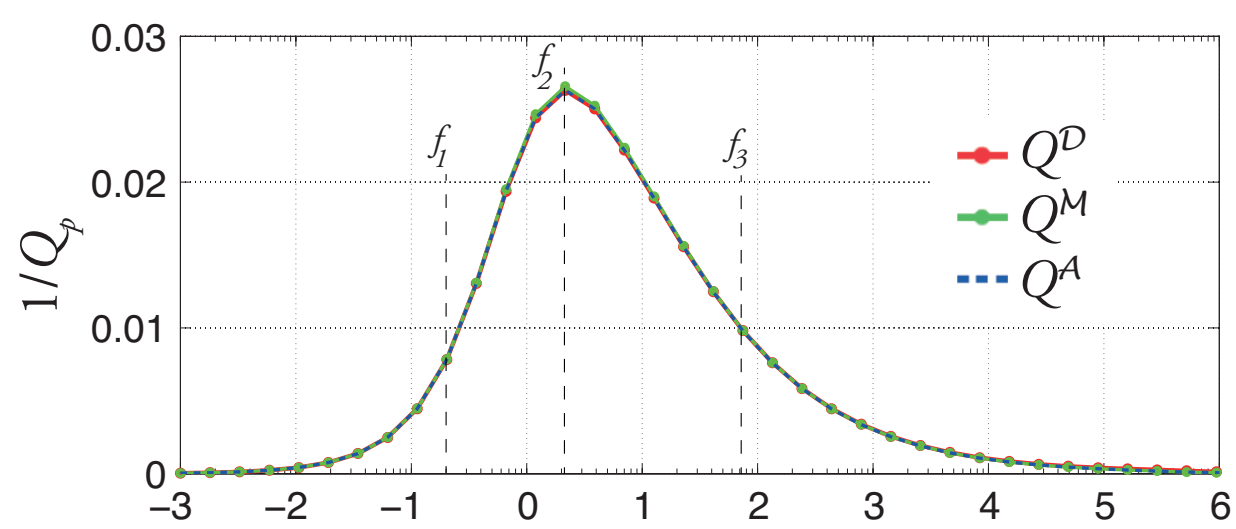

b)

$\log ($ Frecuencia $[\mathrm{Hz}])$

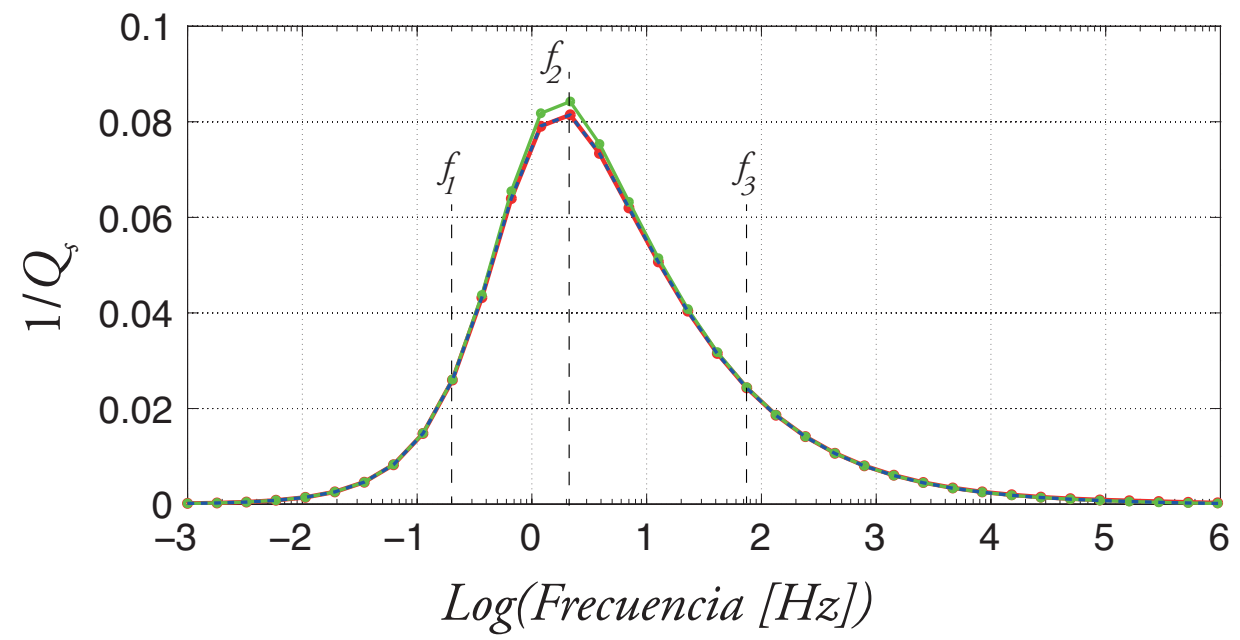

Figura 3.11. Inverso del factor de calidad en función de la frecuencia para la muestra de la Figura 3.10 para el caso de una propagación vertical de una onda (a) P y (b) S. Las curvas corresponden a las diferentes definiciones presentadas en las ecuaciones (3.1), (3.2), y (3.3).

en los extremos de las fracturas (Figuras 3.12 y 3.13). Hemos verificado que esta característica se mantiene para diferentes orientaciones de la fractura dentro de la muestra de roca considerada. Esta particularidad es interesante ya que, como hemos visto previamente, se estima que el máximo de atenuación ocurre en frecuencias $\omega_{c}$ para las cuales la longitud de difusión $L_{d}(\omega)$ es comparable con la longitud característica de la heterogeneidad (ecuación (2.50)). Esto se sostiene para heterogeneidades en las cuales el flujo tiene lugar de manera regular a lo largo de los bordes de las heterogeneidades en la frecuencia crítica, como observamos en el caso de saturación parcial (Figura 3.7b). Sin embargo, el hecho de que para medios fracturados la disipación de la energía se concentre en los extremos de las fracturas (Figuras 3.12 y 3.13) indica que esta relación entre longitud de difusión, longitud característica de la fractura, y frecuencia del máximo de atenuación, puede no ser totalmente correcta para este tipo de escenarios. 


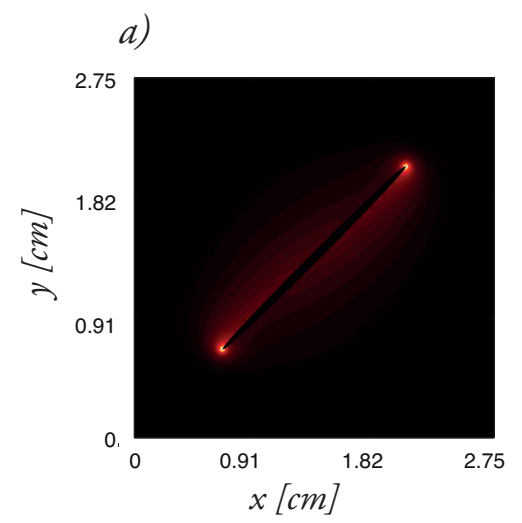

b)

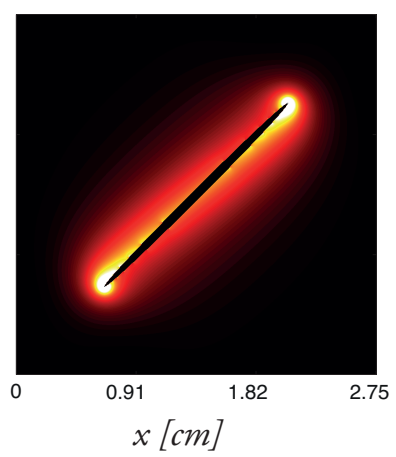

c)

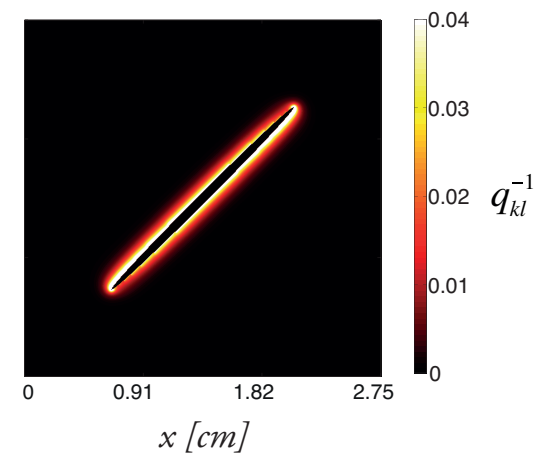

Figura 3.12. Distribución espacial de $q_{k l}^{-1}\left[\mathrm{~m}^{-2}\right]$ en la muestra de la Figura 3.10 para una onda $\mathrm{P}$ propagándose de forma vertical con frecuencias a) $f_{1}=0.2 \mathrm{~Hz}, \mathrm{~b}$ ) $f_{2}=2.15 \mathrm{~Hz}$, y c) $f_{3}=74.44 \mathrm{~Hz}$.

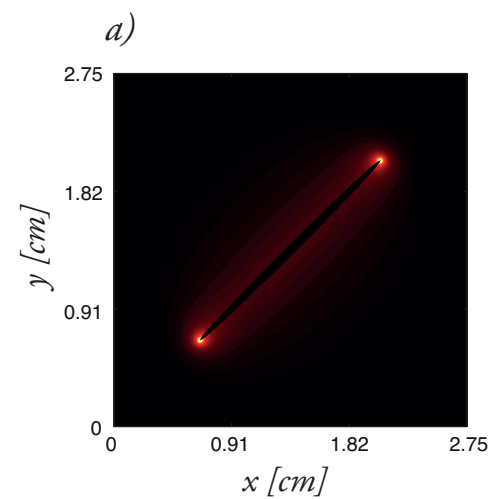

b)

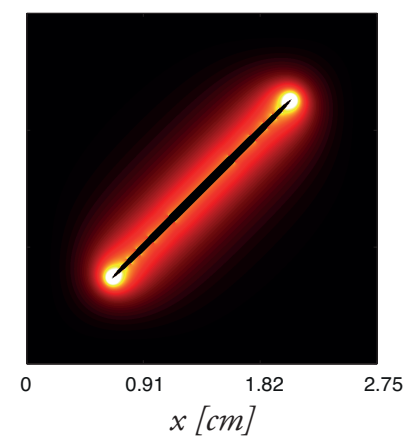

c)

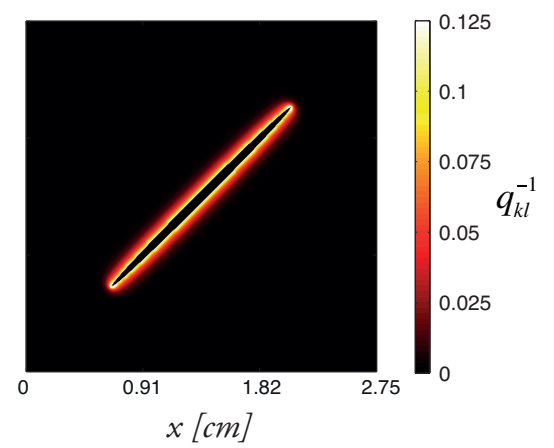

Figura 3.13. Distribución espacial de $q_{k l}^{-1}\left[\mathrm{~m}^{-2}\right]$ en la muestra de la Figura 3.10 para una onda $\mathrm{S}$ propagándose de forma vertical con frecuencias) $f_{1}=0.2 \mathrm{~Hz}, \mathrm{~b}$ ) $f_{2}=2.15 \mathrm{~Hz}$, y c) $f_{3}=74.44 \mathrm{~Hz}$. 


\subsection{Conclusiones}

En este Capítulo hemos propuesto una nueva metodología para calcular la atenuación sísmica debida a flujo inducido por onda en la mesoescala en medios poroelásticos heterogéneos. Esta metodología está basada en el cálculo directo de la potencia disipada y la energía elástica de deformación asociadas al paso de un campo de ondas sísmicas.

Hemos verificado que las estimaciones de atenuación sísmica basadas en la ampliamente utilizada hipótesis de medio viscoelástico equivalente están en acuerdo con aquellas obtenidas a través de una metodología completamente desarrollada en el marco teórico de la poroelasticidad que no hace uso de esta hipótesis. Esto es cierto incluso en escenarios caracterizados por fuertes cambios de compresibilidad, tales como medios parcialmente saturados por «patches» binarios y medios fracturados. En todos los casos considerados en este Capítulo el factor de calidad definido en función de la potencia disipada promedio y la energía de deformación promedio es idéntico a aquel obtenido por medio de la hipótesis de medio viscoelástico equivalente. Al utilizar la energía de deformación máxima para calcular el inverso del factor de calidad observamos ligeras diferencias para valores de atenuación altos. Estos comportamientos, similares a aquellos esperados en sólidos viscoelásticos lineales, le dan sustento a la idea de que la contraparte de un medio poroelástico con heterogeneidades en la mesoescala puede ser representado correctamente por un sólido viscoelástico homogéneo equivalente.

Una característica del método propuesto en este Capítulo, a diferencia de las metodologías que se encuentran en la literatura, es que permite obtener la contribución local a la atenuación global. Esta información adicional puede ser empleada para identificar las regiones que controlan el proceso de disipación, tales como, por ejemplo, los extremos de las fracturas. También puede ser empleado para estudiar la interacción entre procesos de difusión iniciados en heterogeneidades vecinas. Estas dos características permanecen poco exploradas en el contexto del flujo inducido, y pueden permitir el desarrollo de modelos más adecuados para relacionar las características geométricas de las heterogeneidades, la longitud de difusión del proceso de flujo inducido, y las firmas sísmicas asociadas. Consecuentemente, el método propuesto posee la potencialidad de mejorar nuestro actual conocimiento del proceso de flujo inducido por ondas sísmicas en la mesoescala. Las herramientas desarrolladas en este Capítulo serán empleadas en este trabajo de Tesis para analizar la disipación asociada al proceso de flujo inducido en rocas heterogéneas saturadas por distribuciones realistas de fluidos inmiscibles. 


\section{Capítulo 4}

\section{Flujo bifásico en medios porosos he- terogéneos}

Con el objetivo de generar campos de saturación realistas de fluidos inmiscibles que sirvan como escenario de estudio de las propiedades sísmicas, en este Capítulo se introducen las bases teóricas de los procesos de flujo bifásico en medios porosos. En primer lugar, se presentan las ecuaciones y relaciones constitutivas que describen este proceso físico. A su vez, se presenta una formulación que permite modelar el flujo simultáneo de dos fases inmiscibles considerando efectos de capilaridad en medios heterogéneos. Luego, se introducen las bases de un procedimiento de elementos finitos que permite resolver este problema de forma numérica. Por último, se presenta la estructura general del procedimiento numérico desarrollado y su respectiva validación.

\subsection{Introducción}

La mayoría de los trabajos que analizan la respuesta sísmica de medios parcialmente saturados considera que las rocas se encuentran saturadas por distribuciones binarias de fluidos, tales como, por ejemplo, la distribución de fluidos graficada en la Figura 3.5. Sin embargo, experimentos de flujo realizados en rocas porosas heterogéneas, evidencian que los campos de saturación poseen variaciones espaciales continuas, es decir, con valores locales que se encuentran en el rango entre 0 y 1 (p. ej., Shi et al., 2011; Alemu et al., 2013). Por estas razones, es preciso mejorar los modelos de roca parcialmente saturada usualmente empleados al analizar los efectos del flujo inducido sobre las firmas sísmicas. En este Capítulo presentamos un procedimiento computacional que permite generar modelos de roca parcialmente saturada resolviendo las ecuaciones de flujo bifásico.

En primer lugar, es preciso definir la escala de estudio y el tipo de flujo a modelar. En este sentido, se desea generar modelos de flujo que puedan representar de forma adecuada los campos de saturación a escalas mayores que la escala poral. Por lo cual, del mismo modo que al desarrollar la teoría de la poroelasticidad de Biot, se considerará una formulación de medio continuo, donde las leyes de conservación y de equilibrio se basan en la existencia de variables macroscópicas (Bear, 1972). Por otro lado, las fases fluidas involucradas en una gran parte de los escenarios de interés de la Geofísica Aplicada y Ambiental pueden ser consideradas inmiscibles. Tal es el caso del flujo simultáneo de hidrocarburos y agua en un reservorio geológico, o el flujo de contaminantes no acuosos (NAPLs) y agua en un acuífero (Helmig et al., 2006; Hoteit y Firoozabadi, 2008). Por estas razones, es preciso modelar correctamente los efectos característicos del flujo de 
dos fases inmiscibles en un medio poroso heterogéneo, entre los cuales, se destacan los efectos capilares (p. ej., Hoteit y Firoozabadi, 2008).

El modelado numérico de procesos de flujo bifásico en medios porosos heterogéneos es un problema de gran complejidad. Esto se debe a que las ecuaciones diferenciales que describen este proceso físico están acopladas y son altamente no lineales (Chen et al., 2006). Esta dificultad ha llevado a los investigadores a explorar formulaciones alternativas a las ecuaciones de flujo, y a buscar algoritmos especializados que favorezcan su resolución (Binning y Celia, 1999).

El procedimiento clásico de resolución de las ecuaciones de flujo bifásico emplea la formulación de dos presiones (Chavent, 1976). Esta formulación resuelve el sistema de ecuaciones de flujo empleando las presiones de ambas fases como variables principales del sistema. Sin embargo, esta elección de variables presenta problemas si una región se encuentra completamente saturada por una de las fases, ya que la presión de la fase ausente queda indefinida. Para evitar este inconveniente, se suelen manipular y combinar las ecuaciones de flujo, introduciendo funciones auxiliares, logrando así nuevas formulaciones que favorecen la resolución del sistema ante eventuales escenarios de desaparición de una de las fases. Una formulación que cumple estos criterios es la formulación fraccional (p. ej., Chavent et al., 1990). Esta formulación considera a los flujos de las fases individuales como fracciones de un flujo total. Además, considera que las presiones pueden integrarse formando una presión global. De esta manera, la formulación fraccional propone resolver el sistema de ecuaciones empleando como incógnitas principales la saturación de una de las fases y la presión global, permitiendo así modelar problemas que involucran la ausencia de una de las fases (Binning y Celia, 1999).

Como las ecuaciones de flujo bifásico son altamente no lineales, debe emplearse una técnica numérica para su resolución. Existen varias técnicas numéricas desarrolladas para este propósito y, de hecho, la discretización temporal es un tópico de gran interés en la literatura. La gran mayoría de los modelos hidrológicos emplean esquemas completamente implícitos (p.ej., Guarracino y Santos, 2004; Celia y Binning, 1992; Binning y Celia, 1999). Estos esquemas son estables, aunque requieren de una gran cantidad de iteraciones para resolver la no linealidad de las ecuaciones. Por otro lado, en el marco de las simulaciones petroleras, el esquema predominante es un esquema semiimplícito. En este contexto, el esquema más utilizado es implícito en las presiones y explícito en las saturaciones (IMPES). Este procedimiento posee la capacidad de desacoplar las ecuaciones, permitiendo así una resolución secuencial de las ecuaciones de presión y de saturación en cada paso de tiempo (p. ej., Chen et al., 2006; Hoteit y Firoozabadi, 2008; Fuč́k y Mikyška, 2011). Siguiendo el trabajo de Hoteit y Firoozabadi (2008), en este trabajo de Tesis, emplearemos un esquema IMPES sobre las ecuaciones de flujo bifásico.

Por último, existen diversos métodos numéricos desarrollados para resolver las ecuaciones de flujo bifásico. En particular, los métodos de elementos finitos mixtos presentan ciertas ventajas, ya que aproximan la presión y su derivada (el flujo) con la misma precisión, operando sobre ambas variables de forma individual (Guarracino y Santos, 2004). Chavent et al. (1990) emplearon un método de elementos finitos mixto híbrido (EFMH) para resolver el problema de flujo fraccional en medios heterogéneos, considerando una única función de presión capilar. Es importante resaltar que si se consideran 
diferentes funciones de presión capilar dentro del dominio de resolución, la formulación fraccional presenta inconsistencias al ser resuelta empleando un método EFMH. Esto se debe a que tanto la presión global como sus derivadas dejan de ser continuas al considerar diferentes funciones de presión capilar en el dominio de resolución (Hoteit y Firoozabadi, 2008). Mientras que en un medio homogéneo el efecto de la presión capilar puede ser despreciado en ciertos escenarios, este puede no ser el caso en medios heterogéneos. De hecho, la presión capilar está íntimamente relacionada con las propiedades del medio y, por lo tanto, cada heterogeneidad suele estar caracterizada por una función de presión capilar particular (p. ej., Pini et al., 2012; Pini y Benson, 2013). En este contexto, Hoteit y Firoozabadi (2008) propusieron una formulación fraccional alternativa que permite considerar diferentes funciones capilares dentro del dominio de resolución. Esta no utiliza la presión global, sino que toma como incógnitas principales a la presión y a la saturación de una de las fases. Para resolver el sistema de ecuaciones de flujo fraccional alternativo, Hoteit y Firoozabadi (2008) combinaron un método de elementos finitos mixtos híbridos (EFMH) con un método de elementos finitos discontinuos Galerkin (DG). Este último permite describir correctamente los posibles saltos de saturación asociados a medios altamente heterogéneos. Esta combinación de los métodos EFMH-DG posee la ventaja de ser localmente conservativa (Hoteit y Firoozabadi, 2008; Fučík y Mikyška, 2011). Por estas razones, en este trabajo de Tesis utilizaremos la formulación fraccional alternativa y el método de EFMH-DG para generar modelos numéricos de flujo en muestras de roca heterogéneas.

En este Capítulo presentaremos las ecuaciones de flujo bifásico y las relaciones constitutivas en medios porosos. Luego, introduciremos la formulación fraccional alternativa y sus funciones auxiliares. A continuación, emplearemos un esquema IMPES para realizar la discretización temporal del sistema de ecuaciones resultante. Procederemos luego a resolver el sistema de ecuaciones empleando el método de EFMH-DG. Por último, presentaremos una validación del método mediante una comparación de los resultados con la solución analítica de Buckley y Leverett (1942), considerando un medio 1-D homogéneo, y con una solución numérica, en un medio poroso 2-D heterogéneo.

\subsection{Ecuaciones de flujo bifásico en medios porosos}

El flujo bifásico inmiscible en medios porosos está gobernado por dos ecuaciones de conservación de masa y dos ecuaciones de Darcy, una para cada fase fluida. Una de las características de este tipo de flujo es que una de las fases (p. ej., agua) mojará más el medio poroso que la fase restante (p. ej., gas o petróleo). Por lo tanto, las fases fluidas son denominadas fase mojante y fase no mojante, de acuerdo a sus propiedades físicas en relación con la matriz. Si consideramos que tanto la densidad de los fluidos como la porosidad de la matriz no varían en el tiempo, las ecuaciones de conservación de la masa responden a (p. ej., Peaceman, 1977)

$$
\begin{aligned}
& \phi \frac{\partial S_{w}}{\partial t}+\nabla \cdot \mathbf{q}_{w}=0 \\
& \phi \frac{\partial S_{n}}{\partial t}+\nabla \cdot \mathbf{q}_{n}=0,
\end{aligned}
$$


donde los subíndices $w$ y $n$ denotan las fases mojante y no mojante, respectivamente. Además, $S_{\xi}$ y $\mathbf{q}_{\xi}$ denotan la saturación y velocidad de Darcy (o flujo) de la fase $\xi=$ $\{w, n\}$, respectivamente. El flujo de cada fase responde a la ecuación de Darcy:

$$
\begin{gathered}
\mathbf{q}_{w}=-\lambda_{w}\left(S_{w}\right) \kappa \nabla\left(p_{w}-\rho_{w} \mathbf{g} \cdot \mathbf{x}\right), \\
\mathbf{q}_{n}=-\lambda_{n}\left(S_{n}\right) \kappa \nabla\left(p_{n}-\rho_{n} \mathbf{g} \cdot \mathbf{x}\right),
\end{gathered}
$$

donde $p_{\xi}$ y $\rho_{\xi}$ son la presión y la densidad de la fase $\xi=\{w, n\}$, respectivamente. Además, $\mathbf{x}$ es el vector posición, $\mathbf{g}$ el vector de aceleración gravitacional. La movilidad de las fases se define de acuerdo a

$$
\lambda_{\xi}\left(S_{\xi}\right)=\frac{k_{r, \xi}\left(S_{\xi}\right)}{\eta_{\xi}}, \quad \xi=w, n
$$

donde $\eta_{\xi}$, y $k_{r, \xi}\left(S_{\xi}\right)$ son la viscosidad dinámica y la permeabilidad relativa de la fase $\xi$, respectivamente. La permeabilidad relativa $k_{r, \xi}\left(S_{\xi}\right)$ expresa la reducción de la permeabilidad del medio para una fase particular debido a la presencia de una segunda fase fluida en el espacio poral y, como veremos en la Sección 4.2.1, existen diversos modelos para expresar su comportamiento en función de la saturación.

Las saturaciones de las fases están condicionadas por

$$
S_{w}+S_{n}=1 .
$$

A su vez, las presiones de ambas fases están relacionadas por la función de presión capilar $p_{c}\left(S_{w}\right)$ (p. ej., Bear, 1972)

$$
p_{c}\left(S_{w}\right)=p_{n}-p_{w} .
$$

Como veremos a continuación, el comportamiento macroscópico de la presión capilar puede modelarse empleando una relación funcional dependiente de la saturación $S_{w}$.

El sistema de ecuaciones de flujo bifásico consiste de las ecuaciones (4.1) a (4.4), donde las saturaciones y presiones de cada fase se encuentran relacionadas por las ecuaciones (4.6) y (4.7). Por último, la función de presión capilar $p_{c}\left(S_{w}\right)$ y las permeabilidades relativas $k_{r, w}\left(S_{w}\right)$ y $k_{r, n}\left(S_{n}\right)$ están determinadas por las relaciones constitutivas del sistema.

\subsubsection{Relaciones constitutivas}

Al emplear una formulación de medio continuo para expresar las ecuaciones de flujo en medios porosos, es necesario contar con modelos que definan el comportamiento macroscópico de la permeabilidad relativa y la presión capilar.

\section{Presión Capilar}

En las formulaciones de medio continuo, la presión capilar macroscópica $p_{c}$ puede relacionarse con la saturación efectiva de la fase mojante (p. ej., Corey, 1954; Brooks y Corey, 1964; van Genuchten, 1980). Se define la saturación efectiva de la fase mojante como

$$
S_{e, w}=\frac{S_{w}-S_{r, w}}{S_{s, w}-S_{r, w}},
$$


donde $S_{r, w}$ y $S_{s, w}$ son la saturación residual y la saturación máxima de la fase mojante, respectivamente. La saturación efectiva de la fase no mojante responde a $S_{e, n}=1-S_{e, w}$. Los modelos más utilizados para relacionar la presión capilar y la saturación efectiva son los modelos de Brooks y Corey (1964) y de van Genuchten (1980).

El modelo de Brooks y Corey (1964), propone

$$
S_{e, w}\left(p_{c}\right)=\left\{\begin{array}{lll}
\left(p_{c} / p_{d}\right)^{\lambda_{\mathrm{BC}}}, & \text { si } & p_{c}>p_{d} \\
1, & \text { si } & p_{c}<p_{d}
\end{array}\right.
$$

donde $\lambda_{B C}$ es el factor de distribución de tamaño poral, un parámetro que caracteriza la distribución de tamaño de los poros del medio. La presión de entrada $p_{d}$ representa el valor de presión que debe alcanzar la fase no mojante para desplazar a la fase mojante del espacio poral. Por otro lado, el modelo de van Genuchten (1980), considera la siguiente relación entre presión capilar y saturación efectiva

$$
S_{e, w}\left(p_{c}\right)=\left[1+\left(\alpha_{\mathrm{VG}} p_{c}\right)^{n_{\mathrm{VG}}}\right]^{-m_{\mathrm{VG}}},
$$

donde $n_{\mathrm{VG}}=1 /\left(1-m_{\mathrm{VG}}\right)$, mientras que $\alpha_{\mathrm{VG}} \mathrm{y} m_{\mathrm{VG}}$ son parámetros de ajuste.

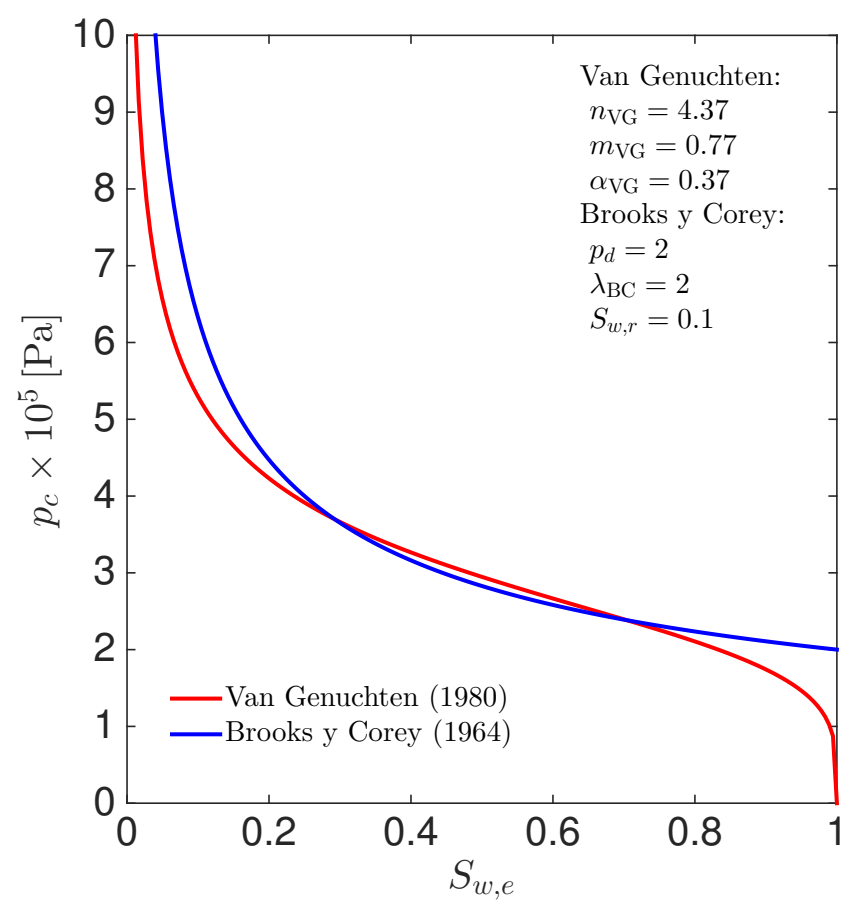

Figura 4.1. Curvas típicas de presión capilar en función de la saturación efectiva. La curva roja responde al modelo de van Genuchten (1980) y la azul al modelo de Brooks y Corey (1964). Los parámetros de ambos modelos han sido tomados de Pavuluri (2014).

La Figura 4.1 ilustra el comportamiento de la presión capilar en función de la saturación efectiva para los modelos de Brooks y Corey (ecuación (4.9)) y de van Genuchten (ecuación (4.10)). Podemos observar que la presión capilar asociada al modelo de Brooks y Corey (curva azul) disminuye a medida que la roca se satura con la fase 
mojante. Al alcanzar el valor máximo de saturación, la presión capilar toma el valor de la presión de entrada $p_{c}\left(S_{s, w}\right)=p_{d}$. En el caso del modelo de van Genuchten (curva roja), también se observa una caída en la presión capilar a medida que $S_{e, w}$ aumenta. Sin embargo, para valores de $S_{e, w}$ cercanos a 1 , es decir para saturaciones cercanas a la saturación máxima de agua, la presión capilar tiende a cero.

\section{Permeabilidad relativa}

Cuando dos fases fluyen simultáneamente dentro un medio poroso, obstruyéndose e impidiéndose el paso entre ellas, la permeabilidad efectiva del medio se ve reducida. La permeabilidad relativa es el parámetro que cuantifica esta reducción en la permeabilidad en las formulaciones de medio continuo. Comúnmente, los procedimientos utilizados para derivar las funciones de permeabilidad relativa están basados en los modelos predictivos de Burdine (1953) o de Mualem (1976).

Es posible derivar, utilizando el modelo predictivo de Burdine (1953), una relación entre la permeabilidad relativa y la saturación, utilizando un determinado modelo de presión capilar $p_{c}\left(S_{w}\right)$. En este contexto, la permeabilidad relativa asociada al modelo de Brooks y Corey (1964), ecuación (4.9), responde a

$$
\begin{gathered}
k_{r, w}\left(S_{w}\right)=S_{e, w}^{\left(2+3 \lambda_{\mathrm{BC}}\right) / \lambda_{\mathrm{BC}}} \\
k_{r, n}\left(S_{w}\right)=\left(1-S_{e, w}\right)^{2}\left(1-S_{e, w}^{\left(2+\lambda_{\mathrm{BC}}\right) / \lambda_{\mathrm{BC}}}\right) .
\end{gathered}
$$

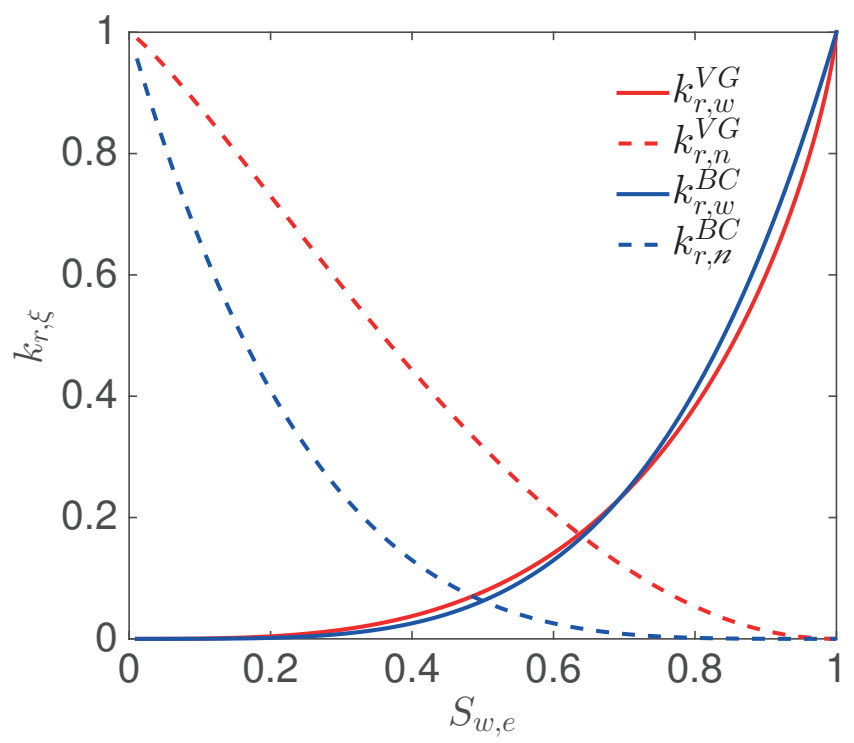

Figura 4.2. Curvas típicas de permeabilidad relativa en función de la saturación. Las curvas rojas responden al modelo de van Genuchten (1980) para la fase mojante (curva continua) y no mojante (curva discontinua). Las curvas azules corresponden al modelo de Brooks y Corey (1964). Los parámetros de ambos modelos han sido tomados de la Figura 4.1. 
Por otro lado, empleando el modelo predictivo de Mualem (1976), la permeabilidad relativa asociada al modelo de van Genuchten (1980), ecuación (4.10), responde a

$$
\begin{aligned}
& k_{r, w}\left(S_{w}\right)=S_{e, w}^{1 / 2}\left(1-\left(1-S_{e, w}^{1 / m_{\mathrm{VG}}}\right)^{m_{\mathrm{VG}}}\right)^{2}, \\
& k_{r, n}\left(S_{w}\right)=\left(1-S_{e, w}\right)^{1 / 2}\left(1-S_{e, w}^{1 / m_{\mathrm{VG}}}\right)^{2 m_{\mathrm{VG}}} .
\end{aligned}
$$

La Figura 4.2 ilustra el comportamiento de la permeabilidad relativa, empleando curvas azules y rojas para las permeabilidades relativas asociadas a los modelos de Brooks y Corey (1964) y van Genuchten (1980), respectivamente. Para diferenciar los comportamientos de la permeabilidad relativa de la fase mojante $k_{r, w}\left(S_{w}\right)$ y de la fase no mojante $k_{r, n}\left(S_{w}\right)$, se utilizan curvas sólidas y curvas a trazos, respectivamente. Se observa que, para ambos modelos, $k_{r, w}\left(S_{w}\right)$ aumenta con la saturación efectiva de la fase mojante, variando entre los valores 0 y 1 . De forma contraria, $k_{r, n}\left(S_{w}\right)$ disminuye de 1 a 0 al aumentar la saturación efectiva.

Una vez definidas las propiedades del medio y de los fluidos, y las relaciones constitutivas, podemos abocarnos a la resolución de las ecuaciones de flujo. Como se ha mencionado previamente, existen diversas formulaciones que permiten plantear el sistema de ecuaciones en función de diferentes variables principales. Entre ellas se destacan: La formulación de dos presiones (p. ej., Chavent, 1976; Celia y Binning, 1992); la formulación fraccional (p. ej., Chavent y Jaffré, 1986; Binning y Celia, 1999); y la formulación fraccional alternativa (p. ej., Hoteit y Firoozabadi, 2008; Fučík y Mikyška, 2011). En este trabajo de Tesis, emplearemos la formulación fraccional alternativa.

\subsection{Formulaciones de las ecuaciones de flujo}

Las ecuaciones de flujo bifásico (4.1) a (4.4) dependen de cuatro variables $S_{w}, S_{n}, p_{w}$, y $p_{n}$. Sin embargo, estas variables están relacionadas. Como veremos a continuación, es posible reducir el problema de flujo bifásico a un sistema de dos ecuaciones con dos incógnitas. La elección de las variables principales está íntimamente relacionada con el problema que se desea resolver. En este sentido, deseamos emplear una formulación que permita modelar correctamente los procesos de flujo en medios porosos heterogéneos en los cuales pueden existir regiones completamente saturadas por la fase mojante.

\subsubsection{Formulación de dos presiones}

La formulación de dos presiones ha sido ampliamente utilizada en la literatura hidrogeológica (p. ej., Celia y Binning, 1992). En ella, las ecuaciones de flujo se escriben en términos de las presiones de cada fase substituyendo las ecuaciones de Darcy (4.3) y (4.4) en las ecuaciones de balance de masa (4.1) y (4.2), es decir,

$$
\begin{array}{r}
\phi \frac{\partial S_{w}\left(p_{c}\right)}{\partial t}-\nabla \cdot\left[\lambda_{w}\left(p_{c}\right) \kappa \nabla\left(p_{w}-\rho_{w} \mathbf{g} \cdot \mathbf{x}\right)\right]=0 \\
\phi \frac{\partial\left(1-S_{w}\left(p_{c}\right)\right)}{\partial t}-\nabla \cdot\left[\lambda_{n}\left(p_{c}\right) \kappa \nabla\left(p_{n}-\rho_{n} \mathbf{g} \cdot \mathbf{x}\right)\right]=0
\end{array}
$$


donde hemos utilizado que $S_{n}=\left(1-S_{w}\left(p_{c}\right)\right)$. El sistema (4.15) utiliza a las presiones $p_{w}$ y $p_{n}$ como variables principales, y debe completarse empleando un juego de relaciones constitutivas.

La elección de las presiones como variables principales no resulta adecuada para modelar problemas donde una de las fases puede desaparecer del medio poroso en una región determinada. Esto se debe a que, si en una determinada región la saturación de una de las fases es nula, la presión asociada queda indefinida (Binning y Celia, 1999). En consecuencia, se han formulado una serie de alternativas al sistema (4.15), tales como, la formulación fraccional.

\subsubsection{Formulación fraccional}

La formulación fraccional tiene sus orígenes en la literatura asociada a la industria petrolera, y se caracteriza por expresar las ecuaciones de flujo en términos de la saturación de la fase mojante y de una nueva variable denominada presión global (p. ej., Chavent y Jaffré, 1986).

En primer lugar se propone sumar las ecuaciones de balance de masa (4.1) y (4.2), lo cual da lugar a

$$
\nabla \cdot\left(\mathbf{q}_{w}+\mathbf{q}_{n}\right)=\nabla \cdot \mathbf{q}_{t}=0
$$

donde $\mathbf{q}_{t}$, comúnmente denominado velocidad total, es la suma de las velocidades de Darcy de ambas fases. Luego, se define una presión global $P_{\mathrm{G}}$, con la intención de expresar la velocidad total en términos de su gradiente. Siguiendo el trabajo de Chavent y Jaffré (1986), la presión global responde a

$$
P_{\mathrm{G}}=\frac{1}{2}\left(p_{w}+p_{n}\right)-\int_{S_{s, w}}^{S_{w}}\left(f_{w}\left(S_{w}\right)-\frac{1}{2}\right) \frac{\mathrm{d} p_{c}\left(S_{w}\right)}{\mathrm{d} S_{w}} \mathrm{~d} S_{w}
$$

donde $f_{w}\left(S_{w}\right)=\lambda_{w}\left(S_{w}\right) / \lambda_{t}\left(S_{w}\right)$ es la función de flujo fraccional, con $\lambda_{t}\left(S_{w}\right)=\lambda_{w}\left(S_{w}\right)+$ $\lambda_{n}\left(S_{w}\right)$. Como se observa en la expresión (4.17), la presión global está íntimamente relacionada con las características de la función de presión capilar. Haciendo uso de la presión global, la velocidad total responde a

$$
\mathbf{q}_{t}\left(P_{\mathrm{G}}, S_{w}\right)=-\kappa \lambda_{t}\left(S_{w}\right) \nabla\left\{P_{\mathrm{G}}-\left[f_{w}\left(S_{w}\right) \rho_{w}+f_{n}\left(S_{w}\right) \rho_{n}\right] \mathbf{g} \cdot \mathbf{x}\right\},
$$

donde $f_{n}\left(S_{w}\right)=\lambda_{n}\left(S_{w}\right) / \lambda_{t}\left(S_{w}\right)$. A su vez, podemos expresar la velocidad de la fase mojante $\mathbf{q}_{w}$ en términos de la velocidad total $\mathbf{q}_{t}$, es decir

$$
\mathbf{q}_{w}\left(P_{\mathrm{G}}, S_{w}\right)=f_{w}\left(S_{w}\right)\left[\mathbf{q}_{t}\left(P_{\mathrm{G}}, S_{w}\right)+\kappa \lambda_{n} \nabla\left(p_{c}\left(S_{w}\right)+\left(\rho_{w}-\rho_{n}\right) \mathbf{g} \cdot \mathbf{x}\right)\right] .
$$

Las ecuaciones de la formulación de flujo fraccional se obtienen reemplazando la ecuación (4.18) en la expresión (4.16), y reescribiendo la ecuación de balance de masa de la fase mojante (4.1) empleando (4.19). Este procedimiento da lugar a

$$
\begin{array}{r}
\nabla \cdot\left(-\kappa \lambda_{t}\left(S_{w}\right) \nabla\left[P_{\mathrm{G}}-\left(f_{w}\left(S_{w}\right) \rho_{w}+f_{n}\left(S_{w}\right) \rho_{n}\right) \mathbf{g} \cdot \mathbf{x}\right]\right)=0 \\
\phi \frac{\partial S_{w}}{\partial t}+\nabla \cdot\left\{f_{w}\left(S_{w}\right)\left[\mathbf{q}_{t}\left(P_{\mathrm{G}}, S_{w}\right)+\kappa \lambda_{n} \nabla\left(p_{c}\left(S_{w}\right)+\left(\rho_{w}-\rho_{n}\right)\right) \mathbf{g} \cdot \mathbf{x}\right]\right\}=0 .
\end{array}
$$

El sistema de ecuaciones (4.20) emplea como variables principales a la presión global $P_{\mathrm{G}}$ y a la saturación de la fase mojante $S_{w}$, por lo cual, suele ser adecuado para modelar 
procesos de flujo en medios heterogéneos donde la fase no mojante puede desaparecer del dominio de resolución. Sin embargo, debido a la dependencia de la presión global $P_{\mathrm{G}}$ con la función de presión capilar $p_{c}\left(S_{w}\right)$ en cada punto del dominio, la formulación fraccional puede presentar problemas al considerar medios heterogéneos con diferentes funciones de presión capilar. Esto se debe a que, en este contexto, $P_{\mathrm{G}} \mathrm{y}$ sus derivadas no serían continuas en el dominio de resolución.

Nuestro objetivo es estudiar el flujo de fases inmiscibles en medios heterogéneos, incluyendo los efectos de capilaridad. Ciertamente, si un medio posee variaciones en sus propiedades petrofísicas, las funciones $p_{c}\left(S_{w}\right)$ serán diferentes. Con la intención de resolver las ecuaciones de flujo en este tipo de escenarios, Hoteit y Firoozabadi (2008) propusieron una formulación de flujo fraccional alternativa, que no utiliza la presión global, sino que toma como variables principales a la presión $p_{w}$ y saturación $S_{w}$.

\subsubsection{Formulación fraccional alternativa}

La formulación fraccional alternativa propone resolver el sistema de ecuaciones (4.1) a (4.4) empleando nuevas velocidades, denominadas $\mathbf{q}_{a}$ y $\mathbf{q}_{c}$, cuyas expresiones responden a (Fučík y Mikyška, 2011)

$$
\begin{gathered}
\mathbf{q}_{a}\left(p_{w}, S_{w}\right)=-\lambda_{t}\left(S_{w}\right) \kappa \nabla\left(p_{w}-\rho_{w} \mathbf{g} \cdot \mathbf{x}\right), \\
\mathbf{q}_{c}\left(S_{w}\right)=-\lambda_{t}\left(S_{w}\right) \kappa \nabla\left[p_{c}\left(S_{w}\right)-\left(\rho_{n}-\rho_{w}\right) \mathbf{g} \cdot \mathbf{x}\right] .
\end{gathered}
$$

Las velocidades de Darcy de las fases mojante $\mathbf{q}_{w}$ y no mojante $\mathbf{q}_{n}$ pueden ser expresadas en términos de las nuevas velocidades $\mathbf{q}_{a}$ y $\mathbf{q}_{c}$, de acuerdo a

$$
\mathbf{q}_{w}=f_{w}\left(S_{w}\right) \mathbf{q}_{a}, \quad \mathbf{q}_{n}=f_{n}\left(S_{w}\right)\left(\mathbf{q}_{a}+\mathbf{q}_{c}\right) .
$$

Luego, se reescribe la ecuación (4.16), empleando las nuevas velocidades,

$$
\nabla \cdot \mathbf{q}_{t}=\nabla \cdot\left(\mathbf{q}_{w}+\mathbf{q}_{n}\right)=\nabla \cdot\left[\mathbf{q}_{a}+f_{n}\left(S_{w}\right) \mathbf{q}_{c}\right]
$$

El sistema de ecuaciones de la formulación fraccional alternativa está basado en las ecuaciones de balance de masa (4.1) y en la ecuación (4.24),

$$
\begin{aligned}
\nabla \cdot\left[\mathbf{q}_{a}\left(p_{w}, S_{w}\right)+f_{n}\left(S_{w}\right) \mathbf{q}_{c}\left(S_{w}\right)\right] & =0 \\
\phi \frac{\partial S_{w}}{\partial t}+\nabla \cdot\left[f_{w}\left(S_{w}\right) \mathbf{q}_{a}\left(p_{w}, S_{w}\right)\right] & =0
\end{aligned}
$$

Este sistema de ecuaciones requiere de un juego de relaciones constitutivas para la presión capilar y la permeabilidad relativa. En el contexto de la formulación fraccional alternativa, las ecuaciones (4.25) y (4.26) son comúnmente denominadas ecuación de presión y ecuación de saturación, respectivamente (p. ej., Fučík y Mikyška, 2011; Hoteit y Firoozabadi, 2008). Esta elección de variables permite modelar procesos de flujo que contemplan la ausencia de la fase no mojante del espacio poral. Además, la presión $p_{w}$ es continua aún considerando varias funciones de presión capilar dentro del dominio de resolución. Por estas razones, consideramos que esta formulación es adecuada para los objetivos planteados en este trabajo de Tesis. 


\subsection{Problema diferencial}

El problema diferencial asociado a la formulación fraccional alternativa responde al siguiente juego de ecuaciones:

$$
\begin{array}{r}
\phi \frac{\partial S_{w}}{\partial t}+\nabla \cdot\left[f_{w}\left(S_{w}\right) \mathbf{q}_{\mathbf{a}}\right]=0, \\
\nabla \cdot\left[\mathbf{q}_{a}+f_{n}\left(S_{w}\right) \mathbf{q}_{c}\right]=0, \\
\mathbf{q}_{a}=-\lambda_{t}\left(S_{w}\right) \kappa \nabla\left(p_{w}-\rho_{w} \mathbf{g} \cdot \mathbf{x}\right), \\
\mathbf{q}_{c}=-\lambda_{t}\left(S_{w}\right) \kappa \nabla\left[p_{c}\left(S_{w}\right)-\left(\rho_{n}-\rho_{w}\right) \mathbf{g} \cdot \mathbf{x}\right] .
\end{array}
$$

La resolución de este sistema está sujeta a condiciones de borde e iniciales que determinan la saturación inicial, y las presiones o los flujos en los bordes externos. Sea $\Gamma=\Gamma^{N} \cup \Gamma^{D}$ el borde del dominio computacional $\Omega$, donde $\Gamma^{N}$ y $\Gamma^{D}$ son bordes no solapados que corresponden a condiciones tipo Neuman y Dirichlet, respectivamente. El sistema de ecuaciones está sujeto a

$$
\begin{aligned}
\mathbf{q}_{\xi} \cdot \boldsymbol{\nu}=q_{\xi}^{N}(\mathbf{x}), & \mathbf{x} \in \Gamma^{N} \subset \partial \Omega, \quad \xi=n, w, \\
p_{w}=p_{w}^{D}(\mathbf{x}), & \mathbf{x} \in \Gamma^{D} \subset \partial \Omega, \\
S_{w}(\mathbf{x}, t=0)=S_{w}^{\text {ini }}(\mathbf{x}), & \mathbf{x} \in \Omega \\
S_{w}=S_{w}^{D}(\mathbf{x}), & \mathbf{x} \in \Gamma^{D} \subset \partial \Omega,
\end{aligned}
$$

donde $\boldsymbol{\nu}$ denota la normal saliente a $\Omega$, y $q_{\xi}^{N}$ y $p_{w}^{D}$ son el flujo de la fase $\xi$ y la presión de la fase mojante en $\Gamma^{N}$ y $\Gamma^{D}$, respectivamente. A su vez, $S_{w}^{\text {ini }}$ es la saturación inicial, y $S_{w}^{D}$ es la saturación del fluido en el borde $\Gamma^{D}$. Las condiciones de borde e iniciales deben ser consistentes con (4.6) y (4.7). En este sentido, al considerar $S_{w}^{D}$ sobre $\Gamma^{D}$, se establece un valor para la presión capilar en ese borde $p_{c}^{D}\left(S_{w}^{D}\right)$. Por lo cual, de forma indirecta, se define la presión de la fase no mojante en este borde $p_{n}^{D}=p_{c}^{D}\left(S_{w}^{D}\right)+p_{w}^{D}$.

El sistema de ecuaciones (4.27)-(4.30) es un sistema no lineal y altamente acoplado. A continuación, implementaremos un esquema numérico IMPES, que permite resolver de forma secuencial las ecuaciones de presión y saturación en cada paso de tiempo (p. ej., Hoteit y Firoozabadi, 2008; Fučík y Mikyška, 2011).

\subsection{Discretización temporal}

Sea $\mathfrak{J}=(0, T)$, con $T>0$ el intervalo de tiempo de interés, con $0=t^{0}<t^{1}<t^{2}<$ $\ldots<t^{N}=T$ una partición de $\mathfrak{J}$. Definiremos un paso temporal $\Delta t^{\mathrm{n}}$ de acuerdo a $\Delta t^{\mathrm{n}}=t^{\mathrm{n}+1}-t^{\mathrm{n}}$, con $\mathrm{n}=0, . ., N-1$. El esquema de discretización seleccionado emplea un algoritmo de Euler hacia adelante, por lo cual, el problema diferencial discretizado responde a

$$
\begin{aligned}
\phi \frac{S_{w}^{\mathrm{n}+1}-S_{w}^{\mathrm{n}}}{\Delta \mathrm{t}^{\mathrm{n}}}+\nabla \cdot\left[f_{w}^{\mathrm{n}}\left(S_{w}^{\mathrm{n}}\right) \mathbf{q}_{a}^{\mathrm{n}}\right]=0, & \mathbf{x} \in \Omega, \\
\nabla \cdot\left[\mathbf{q}_{a}^{\mathrm{n}}+f_{n}^{\mathrm{n}}\left(S_{w}^{\mathrm{n}}\right) \mathbf{q}_{c}^{\mathrm{n}}\right]=0, & \mathbf{x} \in \Omega, \\
\mathbf{q}_{a}^{\mathrm{n}}=-\lambda_{t}^{\mathrm{n}}\left(S_{w}^{\mathrm{n}}\right) \kappa \nabla\left(p_{w}^{\mathrm{n}}-\rho_{w} \mathbf{g} \cdot \mathbf{x}\right), & \mathbf{x} \in \Omega, \\
\mathbf{q}_{c}^{\mathrm{n}}=-\lambda_{t}^{\mathrm{n}}\left(S_{w}^{\mathrm{n}}\right) \kappa \nabla\left[p_{c}^{\mathrm{n}}\left(S_{w}^{\mathrm{n}}\right)-\left(\rho_{n}-\rho_{w}\right) \mathbf{g} \cdot \mathbf{x}\right], & \mathbf{x} \in \Omega,
\end{aligned}
$$


donde el supraíndice $\mathrm{n}$ indica que la variable correspondiente se encuentra evaluada a tiempo $t^{\mathrm{n}}$. A su vez, las condiciones de borde e iniciales responden a

$$
\begin{array}{rr}
\mathbf{q}_{\xi}^{\mathrm{n}} \cdot \boldsymbol{\nu}=q_{\xi}^{N, \mathrm{n}}, & \mathbf{x} \in \Gamma^{N} \subset \partial \Omega, \quad \xi=n, w, \\
p_{w}^{\mathrm{n}}=p_{w}^{D, \mathrm{n}}, & \Gamma^{D} \subset \partial \Omega, \\
S_{w}^{0}=S_{w}^{\text {ini }}, & \mathbf{x} \in \Omega, \\
S_{w}^{\mathrm{n}}=S_{w}^{D, \mathrm{n}}, & \mathbf{x} \in \Gamma^{D} \subset \partial \Omega .
\end{array}
$$

El método de Euler hacia adelante es un método de primer orden lineal de simple aplicación, sin embargo, el error que proviene de hacer una extrapolación lineal nos limita a utilizar pasos temporales pequeños. Debido a que la ecuación de saturación se resuelve de forma explícita, es preciso considerar el criterio de Courant-FriederichsLewy (CFL) para garantizar la estabilidad del esquema (Courant et al., 1928). Este establece que el paso temporal $\Delta \mathrm{t}^{\mathrm{n}}$ debe satisfacer (p. ej., Chavent et al., 1990)

$$
\mathfrak{C}^{\mathrm{n}}=\frac{\Delta \mathrm{t}^{\mathrm{n}}}{2 h} \operatorname{máx}_{\Omega}\left\{\frac{\mathrm{q}_{\mathrm{i}, \xi}^{\mathrm{n}}}{\phi}\right\}<1, \quad \text { con } \xi=w, n, \quad \text { e } i=x, y,
$$

donde $\mathfrak{C}^{\mathfrak{n}}$ es el número de Courant, $h$ la longitud característica de la discretización espacial empleada, y $q_{i, \xi}^{\mathrm{n}}$ son las componentes del vector velocidad de la fase $\xi$ (Dubey, 2010). Este criterio limita el paso temporal en función de la máxima velocidad de Darcy presente en el dominio discretizado.

A continuación plantearemos la discretización espacial del problema empleando un método de EFMH-DG. Se emplea un método EFMH para resolver, a partir de valores de $S_{w}^{\mathrm{n}}$, las ecuaciones (4.35b)-(4.35d), obteniendo así las presiones $p_{w}^{\mathrm{n}}$ y $p_{c}^{\mathrm{n}}$ y los flujos $\mathbf{q}_{a}^{\mathrm{n}} \mathrm{y} \mathbf{q}_{c}^{\mathrm{n}}$. Luego, se utiliza un método de elementos finitos DG para resolver la ecuación de saturación (4.35a). De esta manera se obtienen las saturaciones en el siguiente paso temporal $S_{w}^{\mathrm{n}+1}$. Esto permite realizar una revaluación de los coeficientes a tiempo $t^{\mathrm{n}+1}$ y, de forma iterativa, modelar la evolución del campo de saturación con el tiempo.

\subsection{Discretización espacial}

Consideremos una partición regular no solapada de $\Omega$ en subdominios $\Omega_{j, k}$ de lado $h$, con $j=1, \ldots, N_{x} ; k=1, \ldots, N_{y}$, donde $N_{x}$ y $N_{y}$ representan el número de subdominios en las direcciones $x$ e $y$, respectivamente. La Figura 4.3a ilustra un elemento genérico $\Omega_{j, k}$ de la partición, cuyo borde es $\Gamma_{j, k}=\Gamma_{j, k}^{L} \cup \Gamma_{j, k}^{R} \cup \Gamma_{j, k}^{B} \cup \Gamma_{j, k}^{T}$.

\subsubsection{Resolución de la ecuación de presión}

Utilizaremos un método EFMH para resolver la ecuación de presión. La idea detrás de la aplicación de un método de elementos finitos mixto consiste en estimar de forma conjunta la presión $p_{w}^{\mathrm{n}}$ y la velocidad de Darcy $\mathbf{q}_{a}^{\mathrm{n}}$.

Cuando se emplea un método de elementos finitos estándar o diferencias finitas, el campo de velocidades se suele calcular realizando una diferenciación numérica de la presión $p_{w}^{\mathrm{n}}$. Como consecuencia, si la precisión en la aproximación de $p_{w}^{\mathrm{n}}$ es de orden $\mathcal{O}\left(h^{m}\right)$, la precisión en el campo de velocidades $\mathbf{q}_{a}^{\mathrm{n}}$ es de un orden más bajo $\mathcal{O}\left(h^{m-1}\right)$. La 
aproximación conjunta realizada por los métodos mixtos garantiza que la estimación de la velocidad y de la presión sean del mismo orden. Además, los métodos mixtos garantizan tanto la conservación local como global de la masa. Sin embargo, la complejidad y las dimensiones de los sistemas algebraicos resultantes pueden ser considerables. Lo cual puede representar una limitación computacional importante según el problema a resolver. Esta desventaja puede salvarse hibridizando el problema.

Los procedimiento mixtos híbridos de elementos finitos suelen resultar en sistemas de ecuaciones simétricos y definidos positivos. Esto los hace más simples de resolver que los sistemas asociados a la aplicación de un método mixto de elementos finitos convencional (Hoteit y Firoozabadi, 2008). La hibridización consiste en remover la condición de continuidad de la componente normal de la velocidad en los bordes internos de los elementos del dominio. El requerimiento de continuidad se impone introduciendo un conjunto de multiplicadores de Lagrange, que se encuentran asociados a las presiones $p_{w}^{\mathrm{n}}$ en los bordes de los elementos. Las Figuras $4.3 \mathrm{~b}$ y $4.3 \mathrm{c}$ ilustran los grados de libertad asociados al flujo $\mathbf{q}_{a}^{\mathrm{n}}$ y a la presión de la fase mojante $p_{w}^{\mathrm{n}}$.

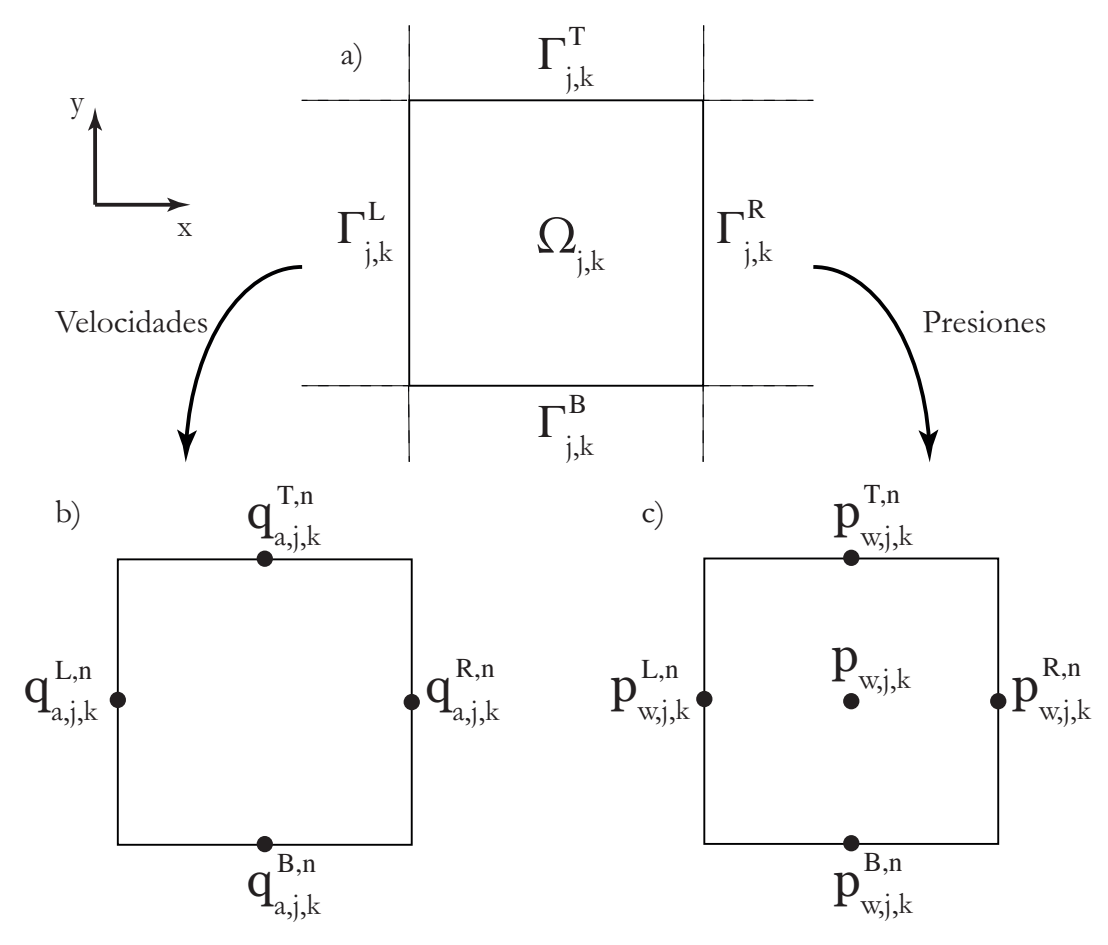

Figura 4.3. Ilustración esquemática de a) un elemento $\Omega_{j, k}$ del dominio. Se ilustran también los grados de libertad asociados a b) la velocidad de Darcy $\mathbf{q}_{a}^{\mathrm{n}}$, y c) a la presión de la fase mojante $p_{w}^{\mathrm{n}}$.

El método EFMH será utilizado para resolver de forma conjunta las ecuaciones (4.35b) y (4.35c). Sin embargo, para llevar a cabo esta tarea, se debe contar con una estimación de $\mathbf{q}_{c}^{\mathrm{n}}$. Para ello debe resolverse, en primer lugar, la ecuación (4.35d).

\section{Aproximación de las velocidades $\mathbf{q}_{c}$}

La ecuación (4.35d) puede ser resuelta de diversas maneras. En particular, podría utilizarse una diferenciación numérica de los valores $p_{c}^{\mathrm{n}}\left(S_{w}^{\mathrm{n}}\right)$ en cada elemento del dominio 
para aproximar la velocidad $\mathbf{q}_{c}^{\mathrm{n}}$. Hoteit y Firoozabadi (2008) propusieron emplear un método de EFMH para obtener una aproximación de mejor calidad para las velocidades. Con este objetivo, se realiza una discretización espacial del campo de velocidades $\mathbf{q}_{c}^{\mathrm{n}} \mathrm{y}$ del campo de presiones $p_{c}^{\mathrm{n}}$ idéntica a la propuesta para $\mathbf{q}_{a}^{\mathrm{n}}$ y $p_{w}^{\mathrm{n}}$, ilustrada en la Figura 4.3. Empleamos funciones lineales asociadas al espacio de Raviart-Thomas-Nédélec de menor orden $\left(\mathrm{RTN}_{0}\right)$ (Raviart y Thomas, 1977; Nedelec, 1980) para aproximar la velocidad y funciones escalares para aproximar el comportamiento de la presión capilar en el centro de los elementos. Las presiones capilares asociadas a los bordes de los elementos resultan de considerar un espacio de multiplicadores de Lagrange. Se impone continuidad en las velocidades y en las presiones capilares en los bordes internos (Hoteit y Firoozabadi, 2008). Haciendo uso de los valores $S_{w}^{\mathrm{n}}$ en el centro de los elementos, es posible encontrar el valor de la presión capilar en los bordes de los elementos del dominio resolviendo un sistema de ecuaciones del tipo

$$
\mathbf{M}_{c}^{\mathrm{n}} \Psi_{c}^{\mathrm{n}}=\mathbf{b}_{c}^{\mathrm{n}}
$$

donde la matriz tridiagonal $\mathbf{M}_{c}^{\mathrm{n}}$ y los vectores $\boldsymbol{\Psi}_{c}^{\mathrm{n}}$ y $\mathbf{b}_{c}^{\mathrm{n}}$ tienen dimensiones iguales al número de bordes de la discretización seleccionada, es decir, $\left(N_{y}+1\right) N_{x}+\left(N_{x}+1\right) N_{y}$. Los componentes del vector $\Psi_{c}^{\mathrm{n}}$ son las presiones capilares en los bordes de los elementos. La matriz $\mathbf{M}_{c}^{\mathrm{n}}$ y el vector $\mathbf{b}_{c}^{\mathrm{n}}$ dependen de las propiedades del medio y de las condiciones de borde del problema. La resolución de este sistema se basa en un procedimiento de eliminación gaussiana, comúnmente denominado Algoritmo de Thomas (p. ej., Conte y De Boor, 1980). Una vez aproximadas las presiones capilares en los bordes de los elementos, es posible aproximar los flujos $\mathbf{q}_{c}^{\mathrm{n}}$.

\section{Aproximación de las velocidades $\mathbf{q}_{a}$}

La resolución conjunta de las ecuaciones (4.35b) y (4.35c) empleando un método EFMH hace uso de las velocidades $\mathbf{q}_{c}^{\mathrm{n}}$ previamente calculadas. Se utilizan funciones lineales asociadas al espacio de $\mathrm{RTN}_{0}$ para aproximar las velocidades $\mathbf{q}_{a}^{\mathrm{n}}$ en cada elemento y funciones escalares para aproximar las presiones $p_{w}^{\mathrm{n}}$. Las presiones de la fase mojante asociadas a los bordes de los elementos resultan de considerar un espacio de multiplicadores de Lagrange. Al asumir continuidad de las velocidades y de las presiones de la fase mojante en los bordes entre elementos, obtenemos un sistema de ecuaciones del tipo

$$
\mathbf{M}_{a}^{\mathrm{n}} \Psi_{w}^{\mathrm{n}}=\mathbf{b}_{a}^{\mathrm{n}}
$$

donde la matriz $\mathbf{M}_{a}^{\mathrm{n}}$ (simétrica y definida positiva), y los vectores $\Psi_{w}^{\mathrm{n}}$ y $\mathbf{b}_{a}^{\mathrm{n}}$ tienen una dimensión igual a la cantidad de bordes de la discretización seleccionada, es decir, $\left(N_{y}+1\right) N_{x}+\left(N_{x}+1\right) N_{y}$. La matriz $\mathbf{M}_{a}^{\mathrm{n}}$ y el vector $\mathbf{b}_{a}^{\mathrm{n}}$ dependen de las propiedades del medio y de las condiciones de borde del problema. $\Psi_{w}^{\mathrm{n}}$ es el vector de incógnitas que contiene las presiones de la fase mojante en los bordes de los elementos. Este sistema de ecuaciones es resuelto de forma iterativa a través de un método de subrelajación por líneas (LSOR) (Peaceman, 1977). Las presiones de la fase mojante en los bordes de los elementos permiten obtener los flujos $\mathbf{q}_{a}^{\mathrm{n}}$ en todo el dominio. 


\subsubsection{Resolución de la ecuación de saturación}

Como se ha resaltado previamente, para resolver la ecuación de saturación (4.35a) empleamos un método de elementos finitos Galerkin discontinuos, considerando una partición del dominio igual a la tomada el resolver la ecuación de presión. Este método asegura la conservación de la masa a escala local y permite la existencia de saltos en la saturación entre elementos (Chen et al., 2006). Se emplean funciones bilineales para aproximar la saturación de la fase mojante en cada elemento. La Figura 4.4 ilustra los grados de libertad asociados a la saturación $S_{w}$ en un elemento genérico $\Omega_{j, k}$. El cálculo del valor de la saturación en cada vértice resulta de resolver un sistema de ecuaciones simple, que depende de los valores de $S_{w}^{\mathrm{n}}$, el valor de $\Delta t^{\mathrm{n}}$, y los flujos $\mathbf{q}_{a}^{\mathrm{n}}$ calculados previamente en los bordes de los elementos. Es importante resaltar que, en las simulaciones realizadas, tomaremos un número de Courant constante $\mathfrak{C}^{\mathrm{n}}=1 / 10, \mathrm{y}$ calcularemos $\Delta t^{\mathrm{n}}$ de forma adaptativa de acuerdo a la ecuación (4.37).

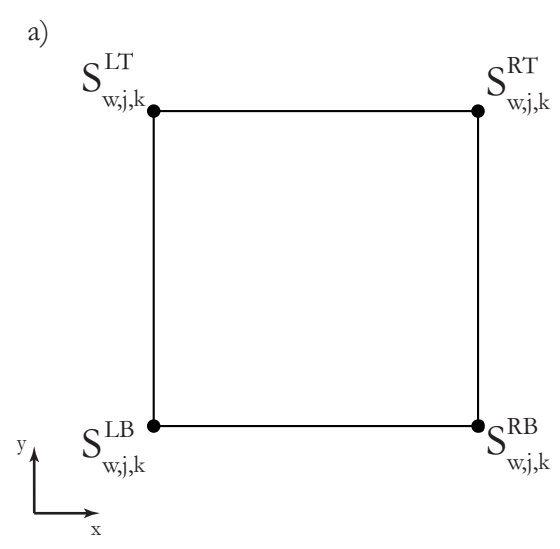

Figura 4.4. Ilustración esquemática de los grados de libertad asociados a la saturación. Los nodos del método de elementos finitos DG se encuentran en los vértices de los elementos.

\subsection{Esquema general de la simulación}

El código computacional para resolver el sistema de ecuaciones por medio del procedimiento EFMH-DG fue desarrollado en lenguaje FORTRAN 90. La estructura del algoritmo puede resumirse en los siguientes pasos:

1. Asignar un valor inicial $t^{0}$, e inicializar el valor del campo de saturación. Asignar un valor de saturación en cada vértice de $\Omega_{j, k}$.

2. Repetir los siguientes pasos hasta alcanzar el tiempo final predeterminado de la simulación $T$ :

a) Calcular, basándose en las saturaciones $S_{w, j, k}^{\mathrm{n}}$ en el centro de cada elemento $\Omega_{j, k}$, las presiones capilares en los centros de los elementos $p_{c, j, k}^{\mathrm{n}}$ utilizando la función de presión capilar correspondiente al elemento en cuestión.

b) Armar la matriz $\mathbf{M}_{c}^{\mathrm{n}}$ y el vector $\mathbf{b}_{c}^{\mathrm{n}}$ correspondientes al sistema (4.38). 
c) Resolver el sistema (4.38) y luego calcular los flujos $q_{c, j, k}^{\mathrm{n}}$ para todos los bordes de $\Omega_{j, k}$

d) Armar la matriz $\mathbf{M}_{a}^{\mathrm{n}}$ y el vector $\mathbf{b}_{a}^{\mathrm{n}}$ correspondientes al sistema (4.39).

e) Resolver el sistema (4.39) y calcular $q_{a, j, k}^{\mathrm{n}}$ para todos los bordes de $\Omega_{j, k}$.

f) Determinar $\Delta t^{\mathrm{n}}$ empleando la ecuación (4.37).

g) Resolver la ecuación de saturación para obtener $S_{w, j, k}^{n+1}$ en los vértices de $\Omega_{j, k}$.

h) Verificar la conservación de la masa empleando las velocidades de Darcy y la variación de la saturación de la fase mojante en cada elemento

i) Avanzar un paso de tiempo $t^{\mathrm{n}+1}=t^{\mathrm{n}}+\Delta t^{\mathrm{n}}$.

\subsection{Validación del método numérico}

En esta sección procedemos a validar el método numérico. Para ello inicialmente se comparan sus salidas con una solución analítica 1-D, resultante de un experimento de flujo realizado en un medio homogéneo, en el cual se desprecian los efectos capilares. Luego, se resuelve un experimento numérico de flujo en un medio heterogéneo 2-D, considerando efectos capilares, y se comparan los resultados con aquellos obtenidos por Hoteit y Firoozabadi (2008) en el mismo escenario.

\subsubsection{Solución analítica de la ecuación de Buckley-Leverett}

En el marco del flujo bifásico una de las soluciones clásicas es la ecuación de Buckley y Leverett (1942). Esta puede obtenerse al resolver las ecuaciones de flujo bifásico en medios porosos para el caso de flujo unidimensional despreciando los efectos de la gravedad, capilaridad, y compresibilidad de los fluidos (Bear, 1972; Chen et al., 2006).

Consideremos entonces un medio poroso 1-D homogéneo sujeto a las siguientes condiciones de contorno

$$
\begin{array}{cll}
S_{w}=S_{w}^{i n i}, & \text { con } \quad t=0, & 0 \leq x \leq L, \\
q_{w}=q_{w}^{N}, & \text { con } \quad t>0, & x=0, \\
q_{n}=0, & \text { con } t>0, & x=0,
\end{array}
$$

donde $S_{w}^{\text {ini }}$ es el estado inicial de saturaciones, dado por la saturación residual de agua, y $L$ la longitud del dominio de estudio. La tasa de inyección de fase mojante $q_{w}^{N}$ es constante en el punto $x=0$.

Considerando la ecuación (4.16) en 1-D, tenemos

$$
\frac{\partial q_{t}}{\partial x}=\frac{\partial\left(q_{w}+q_{n}\right)}{\partial x}=0
$$

es decir, el flujo total es constante, e igual a la tasa de inyección $q_{t}=q_{w}^{N}$.

Si despreciamos los efectos de la presión capilar y la gravedad, y utilizamos las relaciones expresadas en (4.23), es fácil probar que la velocidad de Darcy de la fase 
mojante es igual a: $q_{w}=f_{w}\left(S_{w}\right) q_{t}$. Luego, podemos expresar la ecuación de saturación de la fase mojante (4.1), de acuerdo a

$$
\frac{\partial S_{w}}{\partial t}+\frac{q_{w}^{N}}{\phi} \frac{\partial f_{w}\left(S_{w}\right)}{\partial S_{w}} \frac{\partial S_{w}}{\partial x}=0
$$

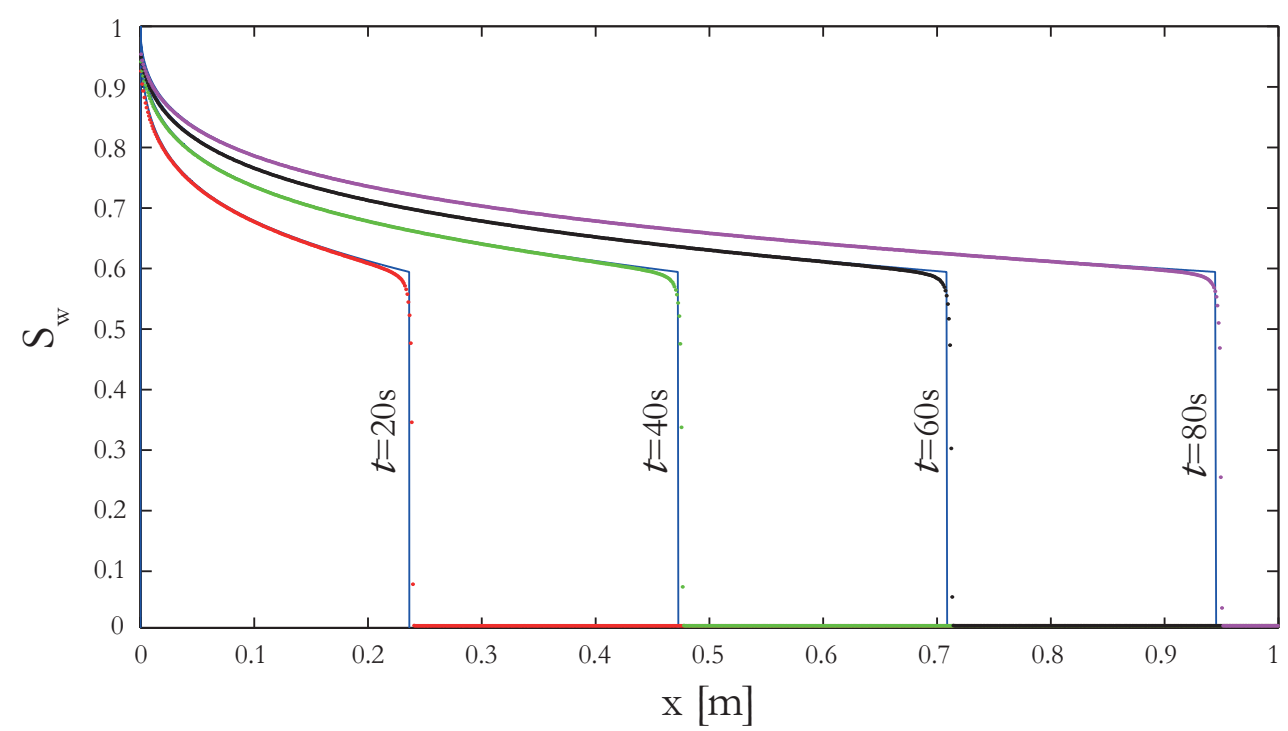

Figura 4.5. Saturación de la fase mojante en función de la posición durante un proceso de inyección a tasa constante. Los perfiles de saturación graficados empleando líneas sólidas azules responden a la solución analítica de Buckley-Leverett, para diferentes tiempos. Las soluciones del método numérico propuesto están dadas por lineas punteadas de diferentes colores para cada tiempo considerado en este análisis.

La ecuación (4.42) es una ecuación diferencial parcial en $S_{w}$ que puede resolverse por medio del método de las características (Bear, 1972). La solución posee la siguiente expresión

$$
\left.x\right|_{S_{w}}(t)=\frac{q_{w}^{N}}{\phi}\left(\frac{\partial f_{w}\left(S_{w}\right)}{\partial S_{w}}\right) t+\left.x\right|_{S_{w}}(0),
$$

donde $\left.x\right|_{S_{w}}(t)$ y $\left.x\right|_{S_{w}}(0)$ son las coordenadas del frente cuya saturación es $S_{w}$ en los tiempos $t$ y 0 , respectivamente. Esta ecuación permite obtener $S_{w}(t)$ en función de $x$ de manera implícita. En particular, la solución (4.43) depende de $\partial f_{w} / \partial S_{w}$. Por lo cual, la solución está condicionada por la elección de las funciones $k_{r, w}\left(S_{w}\right)$ y $k_{r, n}\left(S_{w}\right)$. Debido a las propiedades de $\partial f_{w} / \partial S_{w}$ la solución de la ecuación (4.43) es una función multievaluada. Para salvar esta multievaluación se emplea el método de Welge (1952).

La Figura 4.5 muestra los perfiles de saturación para una medio homogéneo de $1 \mathrm{~m}$ de longitud, inicialmente saturado con un fluido no mojante cuyas propiedades están descriptas en la Tabla 4.1, en el cual se inyecta agua por el borde izquierdo a tasa constante, dada por $q_{w}^{N}=3.57 \times 10^{-3} \mathrm{~m} / \mathrm{s}$. Las curvas azules muestran los perfiles calculados con la ecuación de Buckley-Leverett para diferentes tiempos. A su vez, empleando líneas punteadas, con colores diferentes en cada tiempo, se grafican los perfiles obtenidos con el método numérico. Se ha considerado que las permeabilidades relativas responden a las ecuaciones (4.11)-(4.12). Las propiedades de la matriz, de los 
Tabla 4.1. Propiedades físicas de los materiales sólidos y fluidos empleados en los ejemplos numéricos de flujo. Aquellos parámetros que tengan asignado un valor N/A en el ejemplo 1-D, no son requeridas por el modelo analítico de Buckley y Leverett (1942).

\begin{tabular}{lcc}
\hline & Ejemplo 1-D & Ejemplo 2-D \\
\hline Domino $[\mathrm{m}]$ & 1 & $300 \times 150$ \\
Matriz $[\mathrm{mD}]$ & $\phi=0.488, \kappa=\mathrm{N} / \mathrm{A}$ & $\phi=0.2, \kappa=1,100$ \\
Fluidos $[\mathrm{Pa} . \mathrm{s}]$ & $\eta_{w}=0.001, \eta_{n}=0.0035$ & $\eta_{w}=0.001, \eta_{n}=5 \times 10^{-4}$ \\
$\quad\left[\mathrm{~kg} / \mathrm{m}^{3}\right]$ & $\rho_{w}=\mathrm{N} / \mathrm{A}, \rho_{n}=\mathrm{N} / \mathrm{A}$ & $\rho_{w}=1000, \rho_{n}=660$ \\
$B_{c}\left[\mathrm{~Pa} \mathrm{mD} \mathrm{mD}^{1 / 2}\right]$ & $\mathrm{N} / \mathrm{A}$ & $1 \times 10^{7}$ \\
$\lambda_{B C}$ & 4.66 & 2 \\
Sat. residuales & $S_{w, r}=0 S_{n, r}=0$ & $S_{w, r}=0 S_{n, r}=0$ \\
Tamaño de malla & 200 segmentos & $200 \times 100$ rectángulos \\
\hline
\end{tabular}

fluidos porales, y los valores de los parámetros asociados a las relaciones constitutivas se encuentran en la Tabla 4.1. Puede observarse un muy buen acuerdo entre la solución numérica y la analítica.

\subsubsection{Flujo bifásico en medios heterogéneos 2-D}

El objetivo de esta Sección es comparar nuestras soluciones numéricas con resultados conocidos en medios heterogéneos 2-D. Para ello consideramos un ejemplo analizado en los trabajos de Kou y Sun (2010) y Yang et al. (2016), el cual será descripto a continuación. Estos autores consideran una formulación fraccional alternativa para resolver la ecuaciones de flujo.

Consideremos un dominio de $300 \mathrm{~m} \times 150 \mathrm{~m}$ de permeabilidad $100 \mathrm{mD}$ que contiene dos barreras de baja permeabilidad de $1 \mathrm{mD}$, tal como se muestra en la Figura 4.6a. Específicamente, el campo de permeabilidades responde a

$$
\kappa=\left\{\begin{array}{lr}
1[\mathrm{mD}], & 70 \leq x \leq 100 \text { y } 0 \leq y \leq 100 \\
1[\mathrm{mD}], & 150 \leq x \leq 180 \text { y } 50 \leq y \leq 150 \\
100[\mathrm{mD}], & \text { en el dominio restante }
\end{array}\right.
$$

Inicialmente, el dominio se encuentra completamente saturado por petróleo (fluido no mojante), es decir, $S_{w}^{i n i}=10^{-4}$. Se inyecta agua de manera uniforme a lo largo del borde izquierdo del dominio a una tasa de $q_{w}^{L}=3.8 \times 10^{-7} \mathrm{~m} / \mathrm{s}(0.2$ volúmenes porales por año). Se asume que los bordes superior e inferior del dominio están sellados. A su vez, el borde derecho del dominio se encuentra a presión constante $p_{w}^{R}=0[\mathrm{~Pa}]$. Las propiedades físicas del medio y de los fluidos se encuentran detalladas en la Tabla 4.1.

En el contexto de este ejemplo, las permeabilidades relativas son funciones cuadráticas de la saturación, es decir,

$$
k_{r, w}\left(S_{w}\right)=S_{e, w}^{2}, \quad k_{r, n}\left(S_{w}\right)=\left(1-S_{e, w}\right)^{2} .
$$

Además, la función de presión capilar está dada por

$$
p_{c}\left(S_{w}\right)=-\frac{B_{c}}{\sqrt{k}} \log \left(S_{e, w}\right),
$$


a)

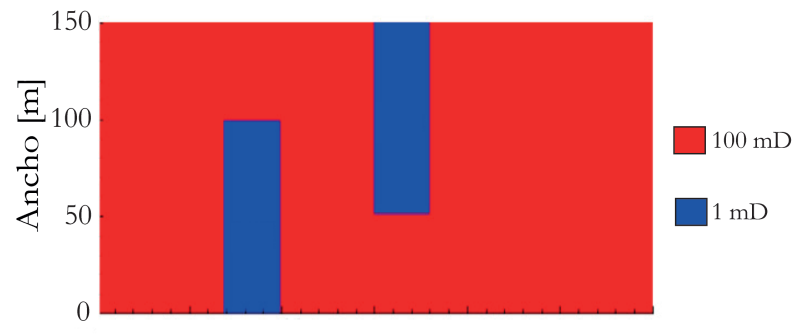

c)

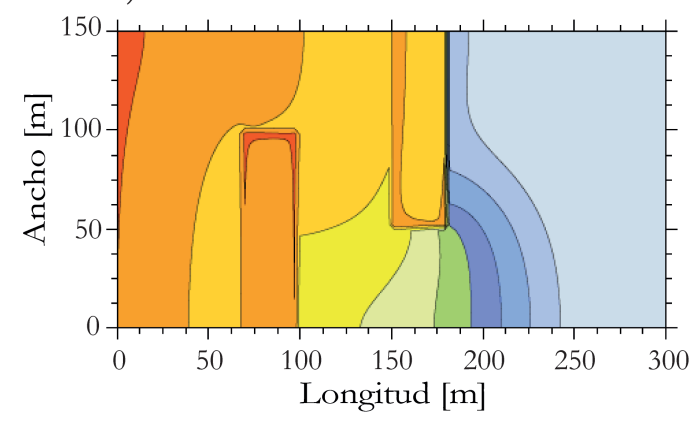

b)

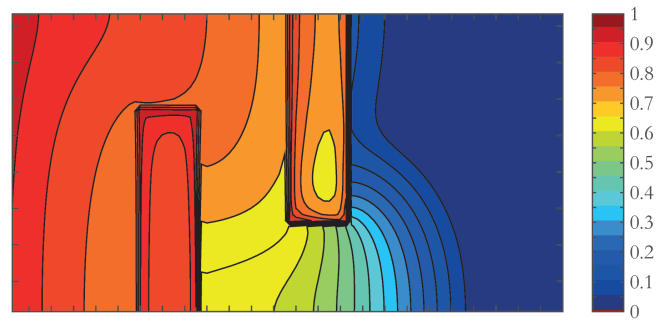

d)

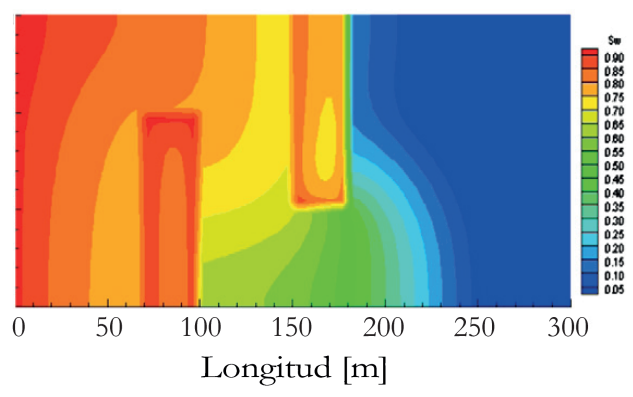

Figura 4.6. a) Dominio 2-D con permeabilidad heterogénea. b) Perfiles de $S_{w}$ a saturación global $50 \%$ obtenidos a través del procedimiento numérico desarrollado. c-d) Perfiles de $S_{w}$ a saturación global $50 \%$ obtenidos por Yang et al. (2016) y Kou y Sun (2010), respectivamente.

donde $B_{c}$ es un parámetro capilar, que en el contexto de este ejemplo es considerado igual a $1 \times 10^{7}\left[\mathrm{~Pa} \mathrm{mD}^{1 / 2}\right]$.

La Figura 4.6b muestra los valores de la saturación de la fase mojante obtenidos con el método numérico desarrollado, para una saturación global de $50 \%$, es decir, luego de 2.5 años de inyección. Se observa que el agua inyectada fluye más rápidamente en las regiones de mayor permeabilidad. Las barreras de baja permeabilidad se saturan, principalmente, debido a los gradientes de presión capilar existentes entre estas y el medio circundante.

Las Figuras 4.6c y 4.6d muestran los valores de la saturación de la fase mojante obtenidos por Yang et al. (2016) y por Kou y Sun (2010) para para el mismo ejemplo, respectivamente. Es importante resaltar que las barras de color utilizadas por estos autores son levemente diferentes a las elegidas en nuestras simulaciones numéricas. Al comparar los resultados de las Figuras 4.6b, 4.6c, y 4.6d, observamos que los resultados son virtualmente idénticos. En ambos casos es posible identificar pequeñas diferencias, posiblemente asociadas a diferencias en los procedimientos numéricos empleados para realizar las inversiones de las matrices y en la discretización temporal. Más allá de esto, los resultados obtenidos confirman que el procedimiento numérico desarrollado logra modelar exitosamente el comportamiento del flujo en medios heterogéneos considerando efectos capilares. 


\subsection{Conclusiones}

En este Capítulo se han presentado las ecuaciones que modelan el flujo simultáneo de dos fases fluidas inmiscibles e incompresibles en un medio poroso heterogéneo. En primer lugar, se introdujo una formulación de las ecuaciones de flujo adecuada al problema que deseamos resolver. Luego, se llevó a cabo una discretización temporal de las mismas través de un esquema IMPES, y se presentó un método EFMH-DG para calcular la evolución del campo de saturación en un medio poroso sujeto a condiciones de borde de presión o de flujo. Finalmente, la técnica numérica propuesta fue validada por medio de la solución analítica de Buckley y Leverett, y por medio de un ejemplo numérico considerando rocas heterogéneas con efectos capilares. A continuación, emplearemos este procedimiento numérico para simular procesos de flujo en rocas porosas heterogéneas, con la intención de estudiar la respuesta sísmica asociada. 


\section{Capítulo 5}

\section{Modelado de atenuación y dispersión de ondas sísmicas durante procesos de imbibición}

La velocidad y la amplitud de las ondas sísmicas suelen experimentar cambios durante procesos de inyección de fluidos en las formaciones exploradas. El análisis de estos cambios podría permitir la delimitación y la caracterización de las zonas afectadas. Sin embargo, para llevar a cabo esta tarea es necesario comprender las relaciones existentes entre los procesos de flujo, la distribución de los fluidos porales, y su efecto en las firmas sísmicas. Con esta motivación, en este Capítulo se estudia la atenuación y la dispersión de ondas sísmicas por flujo mesoscópico durante un experimento de inyección de fluidos. En particular, se propone modelar la inyección de agua en una roca heterogénea inicialmente saturada con gas, emulando un experimento de imbibición realizado en laboratorio. El modelado de flujo emplea la metodología descripta en el Capítulo 4 de este trabajo de Tesis. Luego, analizamos el comportamiento del factor de calidad y de la velocidad sísmica durante el proceso de imbibición empleando las estrategias de cálculo desarrolladas en el Capítulo 3. Este tipo de análisis numérico conjunto permite vincular propiedades inherentes al flujo, tales como, la tasa de inyección y el patrón de saturación resultante, con la atenuación y dispersión sísmica debidas a flujo inducido por onda. Los contenidos del presente Capítulo forman parte del trabajo de Solazzi et al. (2017).

\subsection{Introducción}

Durante un proceso de inyección o de extracción de fluidos en el subsuelo, las fases fluidas involucradas tienden a formar patrones de saturación altamente complejos (p. ej., Alemu et al., 2013). La geometría que presentan estas distribuciones está controlada por las propiedades físicas de las fases fluidas y las propiedades petrofísicas de la matriz, las características geométricas de las heterogeneidades presentes en la formación geológica, el estado inicial de saturación, y las tasas de inyección o extracción de fluidos.

Como se ha observado en los Capítulos 2 y 3 , si los fluidos saturantes poseen compresibilidades contrastantes, el método sísmico no solo es capaz de detectar su presencia, sino que, además, es sensible a las características geométricas de sus patrones de saturación. En este contexto, los experimentos de laboratorio proporcionan oportunidades únicas para ampliar la comprensión actual de los procesos de flujo multifásico, los pa- 
trones de distribución de fluidos resultantes, y su impacto en las firmas sísmicas. En particular, los procesos de imbibición, en los que una fase mojante desplaza una fase no mojante ya presente de los poros de una roca, representa un caso de significativa importancia teórica y práctica. En los últimos años, se ha realizado un considerable esfuerzo en el marco de las Geociencias para comprender, por medio de estudios numéricos y de laboratorio, las características de los procesos de imbibición (p. ej., Lopes y Lebedev, 2012; Lopes et al., 2014; Tisato y Quintal, 2013; Lenormand y Zarcone, 1984; Yin et al., 1992). Si los campos de saturación resultantes de procesos de imbibición presentan «patches» de tamaños mesoscópicos y, además, las compresibilidades de los fluidos involucrados son contrastantes, las ondas sísmicas pueden sufrir atenuación y dispersión por flujo inducido al viajar a través de este tipo de ambientes.

Las evidencias experimentales indican que los patrones de saturación asociados a procesos de imbibición pueden producir niveles significativos de atenuación y dispersión sísmica (p. ej., Tisato y Quintal, 2013; Yin et al., 1992). Discernir las causas de esta disipación no es una tarea fácil. Sin embargo, se ha observado en experimentos de laboratorio que los patrones de saturación generados por imbibición suelen presentar heterogeneidades de mesoescala. Por ello, se intuye que el flujo inducido por onda puede ser un mecanismo de disipación de la energía predominante. A modo de ejemplo, la Figura 5.1 muestra una serie de tomografías computadas obtenidas por Shi et al. (2011) que permiten observar los patrones de saturación durante un experimento de imbibición. En éste, se inyecta agua de formación en una arenisca Tako de $3.7 \mathrm{~cm}$ de diámetro y $14.5 \mathrm{~cm}$ de longitud, inicialmente saturada con $\mathrm{CO}_{2}$. Podemos observar que los fluidos porales toman distribuciones espaciales irregulares, agrupándose en «patches» de tamaños mesoscópicos saturados predominantemente por uno de los fluidos. Es esperable que este tipo de patrones de saturación contribuyan a disipar la energía de las ondas sísmicas por flujo inducido en la mesoescala.

Un parámetro de significativa importancia en los experimentos de imbibición es la tasa de inyección de fluido. Desde una perspectiva hidrológica, los trabajos de Lenormand y Zarcone (1984) y Riaz et al. (2007) reportan que la tasa de inyección de un proceso de imbibición determina, en gran parte, la geometría de la distribución de fluidos. Por otro lado, desde una perspectiva sísmica, se ha observado que los cambios en la tasa de inyección en experimentos de laboratorio tienen efectos significativos sobre la velocidad de las ondas (Lopes y Lebedev, 2012; Lopes et al., 2014). Sin embargo, los detalles de las causas físicas de la respuesta sísmica observada durante procesos de imbibición y, en particular, su relación con la tasa de inyección son actualmente objeto de discusión.

En la actualidad, la interpretación de la información sísmica en el marco del flujo inducido por onda se basa principalmente en modelos analíticos (p. ej., White, 1975; Pride et al., 2004) o procedimientos numéricos de upscaling (p. ej., Masson y Pride, 2007; Rubino et al., 2009). Sin embargo, como hemos visto en capítulos anteriores, estos modelos poseen limitaciones inherentes o han sido utilizados considerando distribuciones de fluidos muy simples. Por ejemplo, los modelos analíticos, tales como el modelo de White (White et al., 1975), consideran rocas saturadas por «patches» binarios de fluidos. Los modelos de upscaling numérico son más flexibles en este sentido, sin embargo, la mayoría de los trabajos consideran campos de saturación binarios basados en procesos estocásticos, tales como los utilizados en el modelo de la Figura 3.5 (p. 


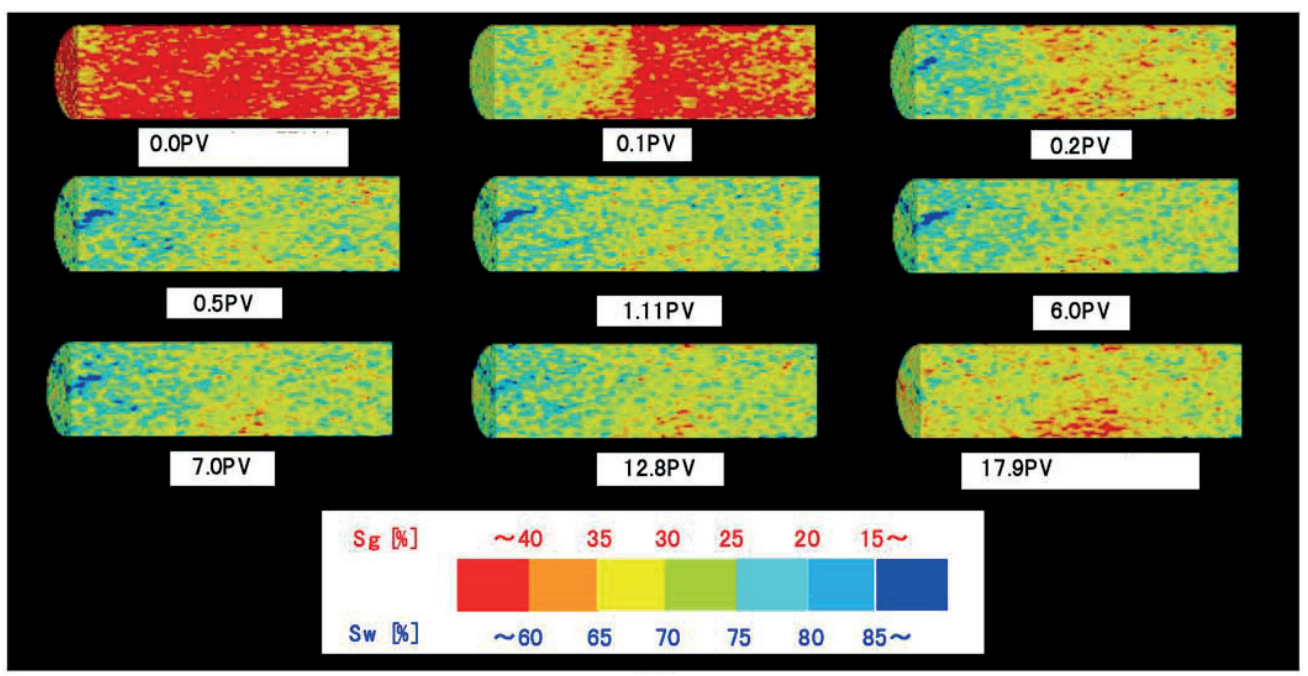

Figura 5.1. Imagen de tomografía computada de la distribución de agua de formación (color azul) y $\mathrm{CO}_{2}$ (color rojo) durante un test de imbibición. Los paneles corresponden a estadíos diferentes del proceso, donde el agua, inyectada desde el extremo izquierdo, satura a la roca con diferentes volúmenes porales (PV). La roca considerada es una arenisca Tako heterogénea de $3.7 \mathrm{~cm}$ de diámetro y $14.5 \mathrm{~cm}$ de longitud. La porosidad media es de 0.27 y el volumen poral de $41.61 \mathrm{~cm}^{3}$. La tasa de inyección, expresada como volumen inyectado por unidad de tiempo y unidad de área, es de $3 \times 10^{-6} \mathrm{~m} / \mathrm{s}$. Este experimento ha sido realizado por Shi et al. (2011).

ej., Rubino et al., 2011; Masson y Pride, 2011). Es importante resaltar que existen razones físicas que nos llevan a cuestionar la existencia de distribuciones binarias de saturación. De hecho, como hemos visto en la Figura 5.1, los estudios experimentales muestran que los campos de saturación, logrados en rocas representativas a partir de procesos de inyección de fluidos inmiscibles, varían de forma suave en el espacio (p. ej., Cadoret et al., 1995; Toms-Stewart et al., 2009).

El modelado numérico de atributos sísmicos utilizando patrones de saturación realistas derivados de leyes de flujo resulta escaso. Esto se debe a que la gran mayoría de los trabajos sobre flujo inducido por onda han estado concentrados en comprender las características del proceso de difusión y su efecto sobre las firmas sísmicas. En consecuencia, se han considerado geometrías simples y/o procesos matemáticos estocásticos para determinar las distribuciones de fluidos. Sin embargo, trabajos recientes han comenzado a considerar modelos más realistas de distribuciones de fluidos. Qi et al. (2014a) modelaron la atenuación sísmica utilizando un modelo de «patches»aleatorio (CRM). La información requerida por el modelo CRM, se obtuvo binarizando imágenes de rayos $\mathrm{X}$ de la distribución de fluidos porales obtenidas durante un proceso de imbibición de laboratorio. Recientemente, Liu et al. (2016) incorporaron variaciones en el tamaño de los «patches» de fluido en el modelo CRM. En particular, estos autores propusieron un modelo para explicar los resultados experimentales de Lopes y Lebedev (2012) y Lopes et al. (2014), donde se midieron velocidades ultrasónicas durante experimentos de imbibición con tasas de inyección variables. Sus resultados sugieren que existe una relación entre la tasa de inyección, el tamaño de los «patches» de fluido, y la respuesta sísmica del medio. Estos trabajos poseen en común que derivan las carac- 
terísticas geométricas de los «patches» binarizando campos de saturación medidos en laboratorio. Como veremos en el Capítulo 6 de este trabajo de Tesis, la binarización de modelos continuos produce consecuencias indeseadas, tales como la sobrestimación de los niveles de atenuación y dispersión. Es importante resaltar que algunos trabajos recientes han abandonado los modelos binarios, y han comenzado a considerar campos de saturación continuos. Rubino y Holliger (2012) exploraron la atenuación sísmica en rocas heterogéneas caracterizadas por campos de saturación continuos de agua y gas. Sin embargo, estos autores asociaron la saturación de la fase mojante con las propiedades de la matriz asumiendo equilibrio capilar. Este trabajo constituye un avance significativo en la cuantificación de la disipación por flujo inducido en medios parcialmente saturados. Sin embargo, la condición de equilibrio capilar aplicada no se satisface durante los experimentos de imbibición realizados en medios heterogéneos. Kuteynikova et al. (2014) modelaron la atenuación sísmica producida por flujo inducido por onda en la mesoescala en rocas homogéneas saturadas con distribuciones continuas de agua y aire. A pesar de considerar modelos simples (capas planas paralelas con transiciones suaves), y desestimar los procesos de flujo asociados a su generación, sus resultados muestran que pequeñas variaciones en la distribución espacial de los fluidos porales pueden cambiar significativamente los valores de atenuación. Para mejorar la comprensión de esta temática, en este Capítulo se realiza un análisis conjunto, considerando procesos de imbibición y cuantificando la atenuación y la dispersión de las ondas sísmicas por flujo inducido.

\subsection{Diseño de modelos numéricos 2-D}

A continuación analizaremos la evolución de la respuesta sísmica de una muestra de roca durante un procedimiento de inyección de fluidos. Como hemos comentado previamente, consideraremos el caso particular de un proceso de imbibición. El mismo consiste en inyectar agua de formación a tasa constante en una arenisca heterogénea, inicialmente saturada con una distribución homogénea de gas metano. Para ello, haremos uso de las herramientas numéricas descriptas en los Capítulos 3 y 4 de este trabajo de Tesis. Comenzaremos por describir las propiedades de la muestra de roca, para luego definir las condiciones del experimento.

\subsubsection{Muestra de roca heterogénea y propiedades físicas aso- ciadas}

Consideremos una muestra sintética de roca $2-\mathrm{D}$ de $36 \mathrm{~cm}$ de altura y $18 \mathrm{~cm}$ de ancho. Las propiedades mecánicas e hidráulicas de la matriz se estiman a partir del campo de porosidad. Empleando la función de densidad espectral del tipo von-Kármán expresada en la ecuación (3.62), generamos un campo de porosidad heterogéneo con variaciones suaves. Consideramos un proceso estocástico con una longitud de correlación isótropa de $1 \mathrm{~cm}$ y un número de Hurst $H=1$. La muestra numérica generada, representada en la Figura 5.2a, será empleada para realizar experimentos numéricos de imbibición. Luego calcularemos la atenuación y dispersión por flujo inducido en la mesoescala. Sin embargo, para evitar que las firmas sísmicas sean influenciadas por la condición 
impuesta en el borde inferior de la roca durante el procedimiento numérico de imbibición, es decir, saturación constante, la atenuación y la dispersión serán estimadas en una submuestra cuadrada de $18 \mathrm{~cm}$ de lado alejada del borde inferior (Figura 5.2b). La distribución de porosidad de esta submuestra posee un valor medio de 0.3 y una desviación estándar de 0.029. La Figura 5.2c muestra el número de celdas en función del valor de porosidad empleando un histograma. Asumiremos que la matriz está compuesta de granos de cuarzo, y que los fluidos considerados en el procedimiento de imbibición son agua de formación y gas metano. Las propiedades físicas de los granos y de los fluidos están expresadas en la Tabla 5.1.

a)

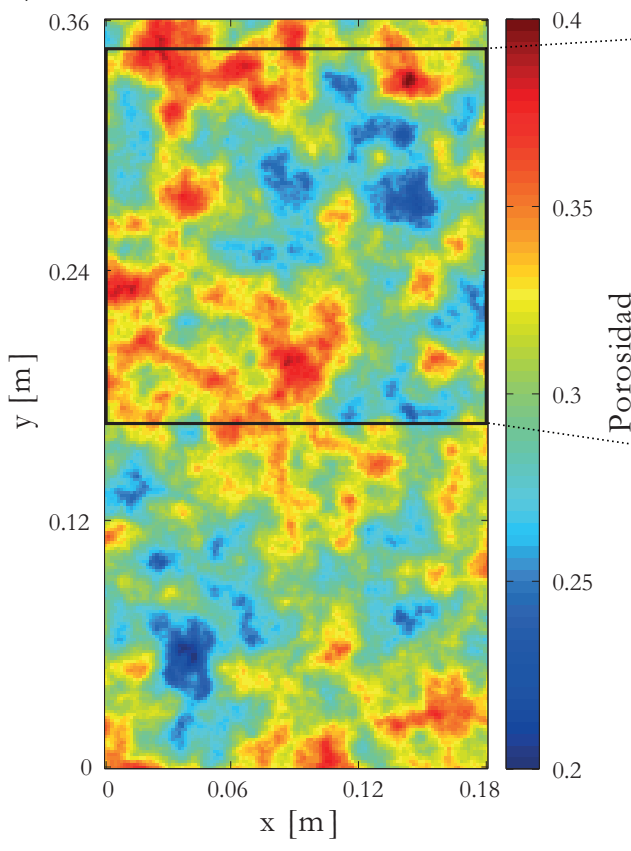

b)
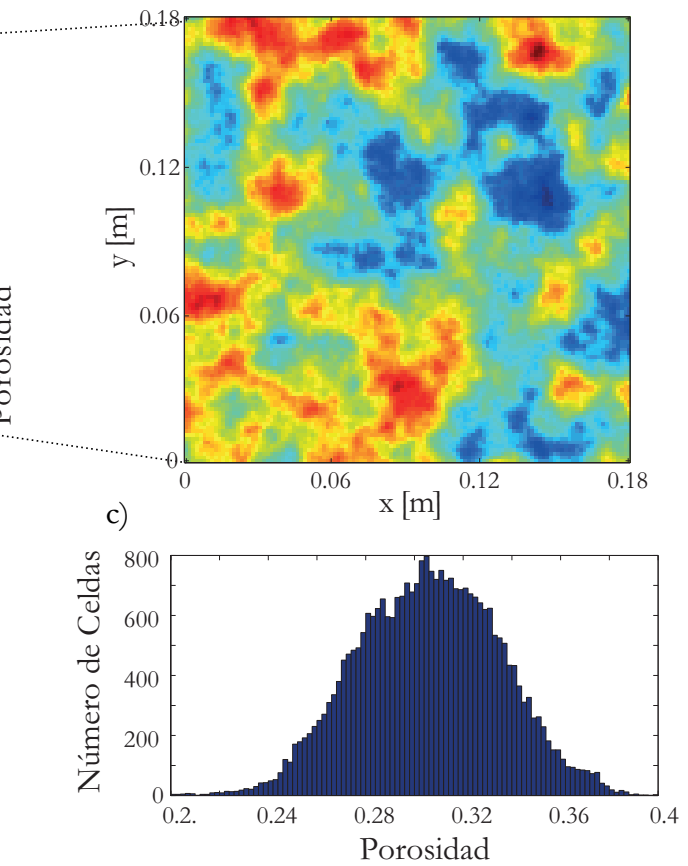

Figura 5.2. (a) Campo de porosidad heterogéneo 2-D considerado en los experimentos numéricos de imbibición y (b) submuestra considerada para analizar la atenuación y dispersión sísmica. (c) Histograma de la porosidad en la submuestra caracterizado por una media de 0.3 y una desviación estándar de 0.029.

Las variaciones en la porosidad implican cambios en otras propiedades físicas de la muestra de roca, incluyendo la permeabilidad, la presión de entrada, y los módulos elásticos de la matriz seca. Utilizaremos la ecuación de Kozeny-Carman para relacionar los valores de porosidad de la roca con la permeabilidad (Kozeny, 1927; Carman, 1937). Esta relación puede ser expresada de acuerdo a (Mavko et al., 2009)

$$
\kappa(\phi)=B \frac{\phi^{3}}{(1-\phi)^{2}} d_{s}^{2},
$$

donde $B=1 / 72 \tau^{2}$ es un factor geométrico que depende de la tortuosidad $\tau$ del medio poroso y $d_{s}$ denota el diámetro medio de los granos sólidos. En las simulaciones numéricas consideraremos una arena cuarzosa con $d_{s}=130 \mu \mathrm{m}$ (p. ej., Renaud et al., 2013). Además, asumiremos $\tau=3.45$, de acuerdo con la tortuosidad aproximada de la 
Tabla 5.1. Propiedades de los materiales considerados en este estudio. Adoptados de Rubino y Holliger (2012) y Rubino et al. (2011).

\begin{tabular}{lccc}
\hline \multicolumn{4}{c}{ Fase sólida } \\
\hline Cuarzo & $K_{s}=37 \mathrm{GPa}$ & $\mu_{s}=44 \mathrm{GPa}$ \\
\hline \multicolumn{3}{c}{ Fases fluidas } \\
\hline Agua & $K_{w}=2.3 \mathrm{GPa}$ & $\eta_{w}=0.001 \mathrm{Pas}$ & $\rho_{w}=1.09 \mathrm{~g} / \mathrm{cm}^{3}$ \\
Gas & $K_{g}=0.012 \mathrm{GPa}$ & $\eta_{g}=0.00015 \mathrm{~Pa} \mathrm{~s}$ & $\rho_{g}=0.078 \mathrm{~g} / \mathrm{cm}^{3}$ \\
\hline
\end{tabular}

arenisca Fontainebleau (Mair et al., 1999), usualmente considerada como un ejemplo representativo de una arenisca cuarzosa limpia.

La presión de entrada $p_{d}$ del modelo de Brooks y Corey (ecuación (4.9)) determina parcialmente el comportamiento capilar del proceso de imbibición. De hecho, la presión de entrada está condicionada por la estructura poral (p. ej., Bear, 1972). En este sentido, el modelo de Leverett determina la presión de entrada de acuerdo a (Leverett, 1941)

$$
p_{d}(\phi)=\gamma \sqrt{\frac{\phi}{\kappa(\phi)}},
$$

donde $\gamma$ es la tensión interfacial entre el agua y el gas. Este parámetro responde a $\gamma \simeq 65 \times 10^{-3} \mathrm{~N} / \mathrm{m}$, de acuerdo con los valores reportados por Ren et al. (2000) para interfases de metano y agua a 10MPa.

Finalmente, asumiremos que los módulos elásticos de la matriz seca pueden expresarse en términos de la porosidad de acuerdo al modelo de Pride (2005)

$$
\begin{gathered}
K_{m}=K_{s} \frac{(1-\phi)}{\left(1+c_{s} \phi\right)}, \\
\mu_{m}=\mu_{s} \frac{(1-\phi)}{\left(1+1.5 c_{s} \phi\right)},
\end{gathered}
$$

donde $K_{s}$ y $\mu_{s}$ denotan el módulo volumétrico y de corte de los granos de cuarzo (Tabla 5.1). El grado de consolidación entre los granos está dado por el parámetro de consolidación $c_{s}$. En el caso de areniscas consolidadas, $c_{s}$ se encuentra entre 2 y 20 (Pride, 2005). Siguiendo el trabajo de Pride y Berryman (2003), asumimos $c_{s}=10$.

A continuación, procederemos a realizar una serie de procesos de imbibición sobre la roca de prueba. Como nuestra intención es simular un proceso de imbibición de laboratorio, debemos determinar condiciones de borde adecuadas para el procedimiento de elementos finitos presentado en el Capítulo 4 de este trabajo de Tesis.

\subsubsection{Modelado del proceso de imbibición}

Utilizaremos el procedimiento descripto en el Capítulo 4 de este trabajo de Tesis para resolver las ecuaciones de flujo bifásico 2-D bajo condiciones inicial y de borde apropiadas, simulando condiciones experimentales de laboratorio para llevar a cabo un proceso de imbibición. Por lo tanto, imponemos una velocidad de Darcy constante $-q_{w}^{T}$ para el agua a lo largo del borde superior de la muestra. A su vez, imponemos una velocidad de Darcy nula para el gas en el borde superior. Tanto el borde izquierdo de la muestra 
como el derecho estarán sellados, es decir, impondremos condiciones de flujo nulo en ellas. En el borde inferior de la muestra impondremos una presión y una saturación de agua constantes. Ambas condiciones definirán de forma implícita a la presión de la fase no mojante. Es importante destacar que estas condiciones de borde son comúnmente utilizadas al realizar experimentos de inyección de fluidos en muestras de rocas de laboratorio (p. ej., Lopes et al., 2014; Pini et al., 2012).

Sea $\Omega=L_{x} \times L_{y}$ el dominio que representa la muestra de roca donde simulamos el proceso de imbibición, y sea $\Gamma$ el borde de $\Omega$, con $\Gamma=\Gamma^{L} \cup \Gamma^{B} \cup \Gamma^{R} \cup \Gamma^{T}$, donde

$$
\begin{aligned}
& \Gamma^{L}=\{(x, y) \in \Omega: x=0\}, \\
& \Gamma^{R}=\left\{(x, y) \in \Omega: x=L_{x}\right\}, \\
& \Gamma^{B}=\{(x, y) \in \Omega: y=0\}, \\
& \Gamma^{T}=\left\{(x, y) \in \Omega: y=L_{y}\right\} .
\end{aligned}
$$

Para resolver las ecuaciones de flujo bifásico, consideraremos una condición inicial de saturación constante

$$
S_{w}^{i n i}=0.08, \quad(x, y) \in \Omega .
$$

Las condiciones de borde responden a

$$
\begin{aligned}
\boldsymbol{q}_{w} \cdot \boldsymbol{\nu} & =-q_{w}^{T}, \quad(x, y) \in \Gamma^{T}, \\
\boldsymbol{q}_{w} \cdot \boldsymbol{\nu} & =0, \quad(x, y) \in \Gamma^{L} \cup \Gamma^{R}, \\
\boldsymbol{q}_{n} \cdot \boldsymbol{\nu} & =0, \quad(x, y) \in \Gamma^{T} \cup \Gamma^{L} \cup \Gamma^{R}, \\
p_{w} & =10 \mathrm{MPa}, \quad(x, y) \in \Gamma^{B}, \\
S_{w} & =0.08, \quad(x, y) \in \Gamma^{B} .
\end{aligned}
$$

La resolución del sistema de ecuaciones (4.27)-(4.30), necesita de relaciones constitutivas que expresen la permeabilidad relativa y la presión capilar como funciones de la saturación de agua. En este sentido, asumiremos que la presión capilar obedece al modelo de Brooks y Corey (1964) (ecuación (4.9)). La presión de entrada está dada por el modelo de Leverett (1941) (ecuación (5.2)). En el caso de areniscas consolidadas, el comportamiento capilar ha sido modelado con valores de $\lambda_{\mathrm{BC}}$ entre 1.4 y 2.7 (Pini y Benson, 2017). Luego, para las simulaciones numéricas consideradas en esta sección tomaremos $\lambda_{\mathrm{BC}}=2$. Además, la saturación residual del agua será $S_{w r}=0.05$, de acuerdo con la saturación irreducible de agua en la arenisca Fontainebleau (Mavko et al., 2009). Finalmente, tomaremos el modelo de Brooks y Corey (1964) (ecuaciones (4.11)-(4.12)) para determinar las permeabilidades relativas $k_{r, w}\left(S_{w}\right)$ y $k_{r, n}\left(S_{w}\right)$.

El método MHFE-DG propuesto en el Capítulo 4, junto con las relaciones constitutivas, y las condiciones de borde aquí definidas, nos permitirá estimar los campos de saturación y su evolución dentro de la muestra de prueba, representada en la Figura 5.2a.

A continuación, describiremos el procedimiento de cálculo de la atenuación y dispersión sísmica seleccionado para este análisis. 


\subsubsection{Cálculo de atenuación y dispersión sísmica por flujo inducido por onda}

Como se ha comentado previamente, nuestro interés yace en estudiar el efecto que producen patrones de saturación realistas, resultantes de procesos de imbibición, sobre las ondas sísmicas. Para ello, saturaremos la submuestra de prueba, representada en la Figura 5.2b, con las distribuciones de fluido obtenidas en las simulaciones de flujo en ciertos estadíos de interés. Luego, aplicaremos el test de upscaling numérico descripto en el Capítulo 3, es decir, someteremos a la roca a un test oscilatorio de relajación. Este procedimiento involucra la resolución de las ecuaciones de consolidación de Biot (2.46)-(2.47) y, a través de ellas, obtener el módulo de onda plana equivalente del medio saturado utilizando la hipótesis de medio viscoelástico equivalente (3.30). Finalmente, utilizando los valores de $M_{c}$ para distintas frecuencias de oscilación, calculamos el inverso del factor de calidad $Q_{p}^{-1}$ a través de la ecuación (3.1).

Por otro lado, para calcular la velocidad de fase equivalente de la onda compresional, utilizamos (p. ej., Rubino et al., 2009)

$$
V_{p}(\omega)=\left[\Re\left\{\sqrt{\frac{\left\langle\rho_{b}\right\rangle}{M_{c}(\omega)}}\right\}\right]^{-1},
$$

donde $\left\langle\rho_{b}\right\rangle$ denota la densidad promedio del agregado.

Como hemos visto en el Capítulo 2 de este trabajo de Tesis, las ecuaciones de Biot (Biot, 1941) consideran localmente la existencia de un único fluido poral. Sin embargo, durante un experimento de imbibición, cada celda de la roca numérica puede estar saturada por ambas fases, agua y gas. Con la intención de resolver esta limitación, emplearemos una fase fluida efectiva local al resolver las ecuaciones (2.46)-(2.47). Es decir, siguiendo el trabajo de Rubino y Holliger (2012), en cada celda computacional definiremos las propiedades de un fluido efectivo considerando las propiedades de las fases individuales pesadas por los valores de saturación respectivos. Luego, la densidad del fluido efectivo está dada por

$$
\rho_{f}=S_{w} \rho_{w}+\rho_{n}\left(1-S_{w}\right) .
$$

Otro parámetro clave en el proceso de flujo inducido por onda es el módulo de volumen del fluido efectivo. Como consideramos celdas cuyos tamaños son mucho menores que las longitudes de difusión involucradas en el proceso de flujo inducido, las perturbaciones en la presión poral generadas por el paso del campo de onda sísmica tienen suficiente tiempo como para equilibrarse dentro de cada celda computacional. Luego, la presión de fluido dentro de cada celda puede asumirse uniforme y podemos utilizar la ley de Wood (Wood, 1955) para obtener el módulo volumétrico del fluido efectivo

$$
K_{f}=\left(\frac{S_{n}}{K_{n}}+\frac{S_{w}}{K_{w}}\right)^{-1}
$$

donde $K_{n}$ y $K_{w}$ son los módulos de volumen del gas y del agua, respectivamente.

Por otro lado, siguiendo el trabajo de Rubino y Holliger (2012), utilizaremos la relación de Teja y Rice (1981) para obtener la viscosidad del fluido efectivo

$$
\eta_{f}=\eta_{n}\left(\frac{\eta_{w}}{\eta_{n}}\right)^{S_{w}}
$$


Es importante remarcar que, a pesar de considerar efectos capilares al resolver las ecuaciones de flujo bifásico, estos efectos no serán tenidos en cuenta al cuantificar el flujo inducido por onda a escala mesoscópica ya que se asume un único fluido con propiedades efectivas.

\subsection{Análisis de la atenuación y dispersión sísmica durante un experimento de imbibición}

En primer lugar sometemos a la muestra de roca, Figura 5.2a, a un experimento de imbibición, caracterizado por una tasa de inyección constante $q_{w}^{T}=1 \times 10^{-4}[\mathrm{~m} / \mathrm{s}]$, hasta llegar a un estado estacionario en la submuestra seleccionada. Consideramos un campo de saturación inicial homogéneo de gas, con un valor de saturación de agua de $8 \%$. El mismo se encuentra por encima de la saturación residual considerada en este análisis (5\%). Como ha sido mencionado previamente, analizaremos la evolución de las distribuciones de gas y agua y de las firmas sísmicas asociadas a la submuestra representada en la figura Figura 5.2b.

\subsubsection{Estimación de la atenuación}

Los gráficos de la Figura 5.3a muestran la evolución de los patrones de saturación que resultan del proceso de imbibición estudiado. Para caracterizar esta evolución, calculamos, en cada caso, la saturación global de la submuestra

$$
\left\langle S_{w}\right\rangle=\frac{\sum_{i j} S_{w}\left(\Omega_{i, j}\right) \phi\left(\Omega_{i, j}\right)}{\sum_{i j} \phi\left(\Omega_{i, j}\right)},
$$

con $\Omega_{i, j}$ denotando el elemento $i j$-ésimo de la malla computacional empleada. A estadíos tempranos del experimento de imbibición se observa una interfase de separación entre las zonas donde el agua ha ingresado y aquellas saturadas mayormente por gas. Esta interfase es conocida como «frente de saturación», y tiende a desaparecer de los gráficos cuando el proceso de imbibición afecta a toda la muestra. Observamos en la Figura 5.3a que el frente de saturación avanza a medida que la saturación global de la muestra aumenta de $\left\langle S_{w}\right\rangle=30 \%$ a $70 \%$. En detalle, se puede observar que, mientras el agua invade las diferentes regiones de la muestra heterogénea, los valores locales de saturación aumentan y la presión capilar disminuye, siguiendo la ecuación (4.9). Como cada celda que compone a la roca numérica se encuentra caracterizada por una porosidad, permeabilidad, y presión de entrada particular, el frente y el campo de saturación detrás de él son heterogéneos. Debido a la distribución espacial heterogénea de los fluidos porales, las diferentes regiones de la roca poseen compresibilidades diferentes. Por esta razón, las ondas sísmicas pueden sufrir disipación por flujo inducido por onda al propagarse por la roca de prueba. Para explorar este proceso, el panel inferior de la Figura 5.3a muestra, para los estadíos del proceso de imbibición previamente descriptos, la atenuación sísmica asociada en función de la frecuencia. Es posible observar que, efectivamente, las ondas sísmicas evidencian disipación por flujo inducido por onda en la mesoescala. Una inspección mayor de estas curvas permite identificar dos máximos de atenuación: el primero se encuentra localizado entre las frecuencias 10 y $100 \mathrm{~Hz}$ y 
a)

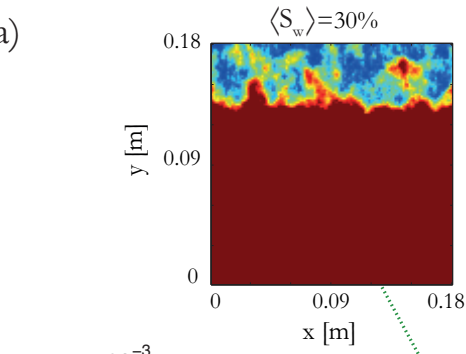

$\left\langle\mathrm{S}_{\mathrm{w}}\right\rangle=30 \%$

$\left\langle\mathrm{S}_{\mathrm{w}}\right\rangle=50 \%$

$\left\langle\mathrm{S}_{\mathrm{w}}\right\rangle=70 \%$

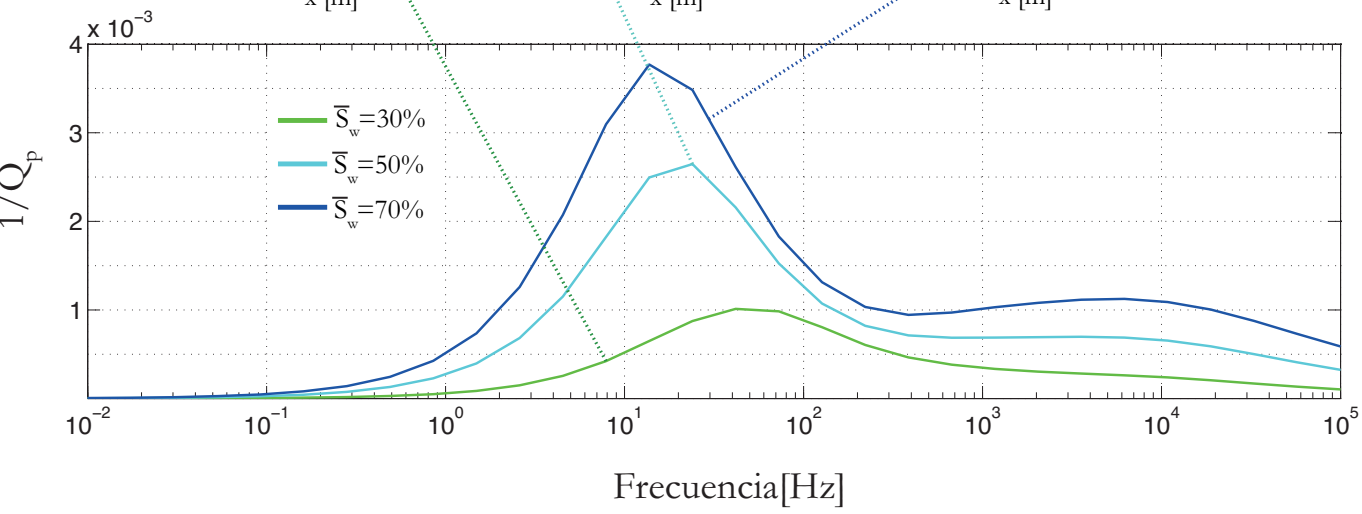

b)

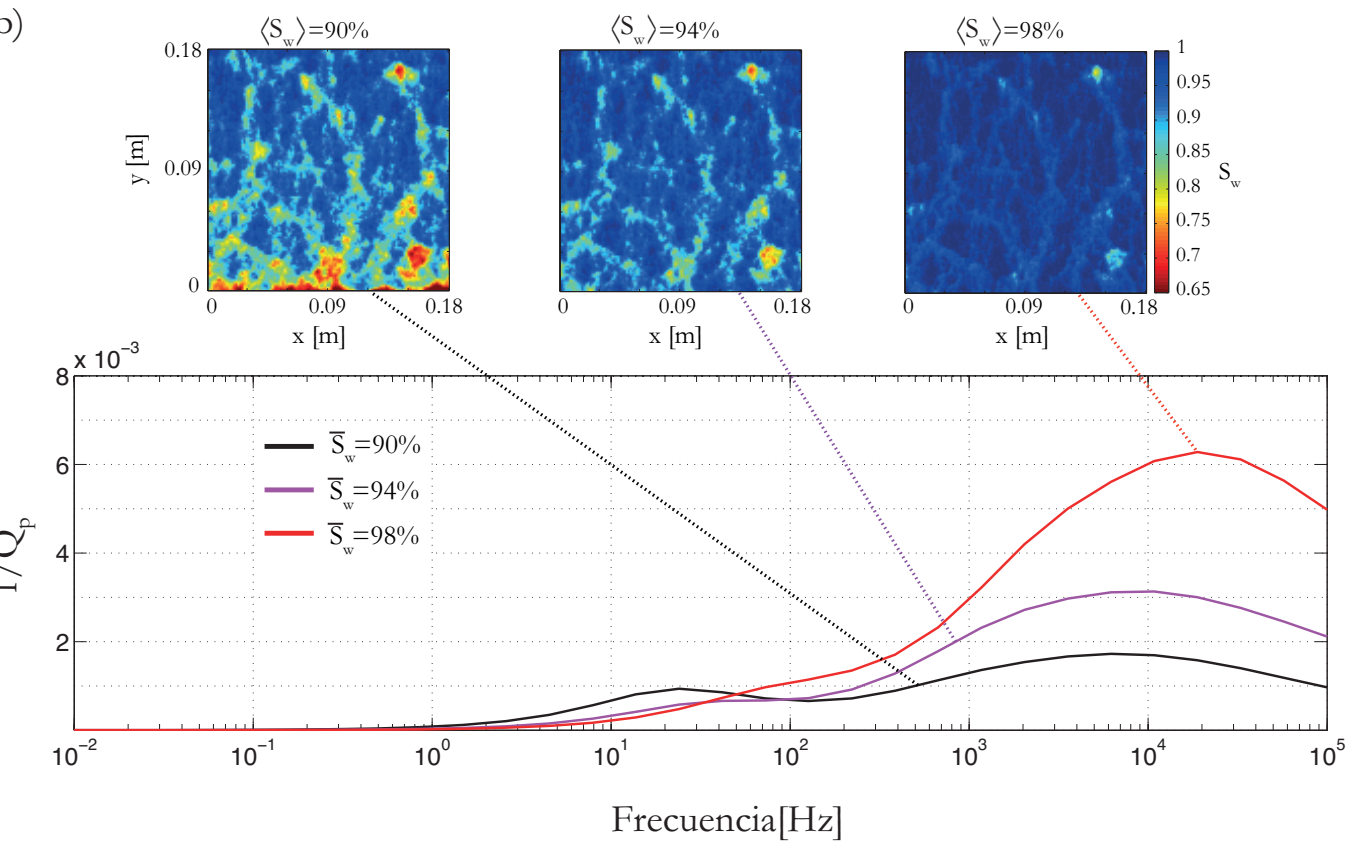

Figura 5.3. Campos de saturación de agua resultantes del procedimiento de imbibición numérico realizado con una tasa de inyección constante $q_{w}^{T}=1 \times 10^{-4}[\mathrm{~m} / \mathrm{s}]$ e inverso del factor de calidad $1 / Q_{p}$ en función de la frecuencia. (a) Las curvas color verde, azul claro, y azul oscuro denotan niveles de saturación global de $30 \%$, $50 \%$ y $70 \%$, respectivamente; (b) Las curvas color negro, violeta, y rojo denotan saturaciones globales de $90 \%, 94 \%$ y $98 \%$, respectivamente.

el segundo alrededor de los $6 \mathrm{kHz}$. Observamos que, al aumentar los valores de saturación global, los niveles de atenuación crecen para ambos máximos. Además, el primer máximo se desplaza hacia frecuencias menores cuando la saturación global aumenta.

Por otro lado, la Figura 5.3b muestra los resultados de las simulaciones a estadíos 
tardíos del proceso de imbibición, para los cuales el frente de saturación ya ha pasado a través de la submuestra. Observamos en los paneles superiores que ciertos «patches» de gas han quedado atrapados detrás del frente de saturación y que, a su vez, hay regiones que poseen altos valores de saturación de agua en sus alrededores. El panel inferior de la Figura 5.3b indica que para estos estadíos del procesos de imbibición, el primer máximo de atenuación es virtualmente inexistente, mientras que el segundo se hace más fuerte que en estadíos previos del proceso de inyección. Además, al aumentar la saturación global, el segundo máximo de atenuación toma valores mayores y se desplaza hacia frecuencias mayores.

Si comparamos las curvas de atenuación de las Figuras 5.3a y 5.3b, podemos relacionar el primer máximo de atenuación con la presencia del frente de saturación mientras que el segundo puede asociarse con la existencia de «patches» residuales detrás del frente de saturación. Como el máximo en bajas frecuencias desaparece cuando el frente abandona la muestra, tendemos a asociar este proceso difusivo al contraste de compresibilidades generado por el frente de saturación. En este sentido, el frente de saturación puede generar un cambio en las propiedades del medio, generando una distribución espacial similar a la de un modelo de doble capa de White (White et al., 1975). Por otro lado, el máximo de alta frecuencia tiende a crecer cuando aparecen regiones con valores altos de saturación de agua rodeados por zonas que contienen una combinación de agua y gas en el espacio poral. En este contexto, es interesante notar, realizando una comparación cualitativa, que los campos de saturación poseen características similares a los patrones de saturación observados en el laboratorio por Shi et al. (2011) (ver Figura 5.1), considerando que en dicho experimento la dirección de inyección es horizontal.

Las simulaciones numéricas muestran que las distribuciones de saturación que resultan de los experimentos de imbibición producen niveles de atenuación sísmica relativamente bajos. A pesar de que no hay consenso claro sobre el menor nivel de atenuación medible con la tecnología actual en experimentos de laboratorio, de acuerdo a Tisato y Madonna (2012), es posible medir la atenuación extensional $1 / Q_{E}$ para valores mayores a 0.003. Por otro lado, los trabajos de Winkler y Nur (1979) y Spencer y Shine (2016) nos permiten concluir que $1 / Q_{p}>1 / Q_{E}$ para muestras parcialmente saturadas. Esto implica que los niveles de atenuación obtenidos en este ejemplo son, en el mejor de los casos, medibles experimentalmente para la saturación global $\left\langle S_{w}\right\rangle=70 \%$ para el primer máximo (Figura 5.3a) y en los casos $\left\langle S_{w}\right\rangle=94 \%$ y $\left\langle S_{w}\right\rangle=98 \%$ para el segundo máximo (Figura 5.3b).

\subsubsection{Estimación de la dispersión sísmica}

El análisis de la variación de la velocidad con el contenido de fluido es un tópico ampliamente estudiado en el contexto de la Física de Rocas (p. ej., Murphy, 1984; Le Ravalec et al., 1996; Lebedev et al., 2009; Lopes y Lebedev, 2012; Alemu et al., 2013; Lopes et al., 2014; Zhang et al., 2015). Esto se debe a que una mayor comprensión de esta relación podría ayudar a estimar el contenido de fluidos de una formación de manera remota. Sin embargo, las medidas de laboratorio suelen realizarse en rangos de frecuencias mayores a los de la sísmica de exploración. Por lo tanto, sería de gran utilidad contar con modelos que permitan representar el comportamiento de la velocidad con 
la frecuencia y el grado de saturación, para extrapolar los efectos dispersivos observados al rango de frecuencias de interés. A continuación analizaremos la dispersión que sufren las ondas sísmicas por flujo mesoscópico en la roca bajo estudio (Figura 5.2b) para diferentes estadíos del proceso de imbibición.

a)

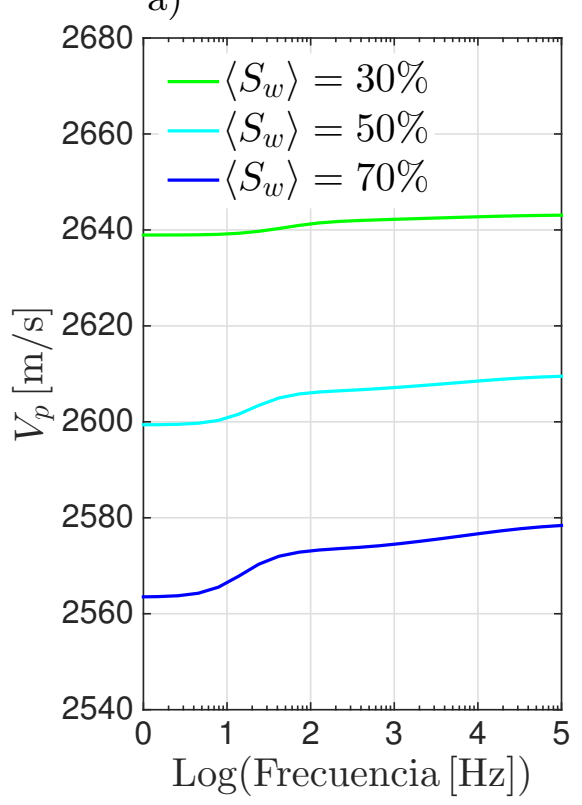

b)

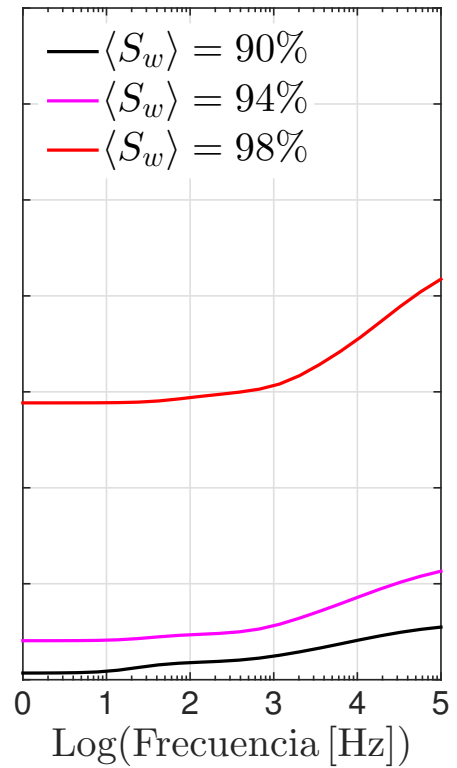

Figura 5.4. Velocidad de fase compresional en función de la frecuencia para (a) estadíos tempranos y (b) estadíos tardíos del proceso de imbibición. Los colores y niveles de saturación se corresponden con aquellos de la Figura 5.3a y Figura 5.3b.

La Figura 5.4a muestra la velocidad en función de la frecuencia para estadíos tempranos del proceso de imbibición. En particular, se grafican las velocidades asociadas a los paneles superiores de la Figura 5.3a, es decir, para saturaciones globales $\left\langle S_{w}\right\rangle=30 \%,\left\langle S_{w}\right\rangle=50 \%, \mathrm{y}\left\langle S_{w}\right\rangle=70 \%$. Podemos observar que, en todos los casos, la velocidad aumenta con la frecuencia. Esto se debe a que la roca tiende a ser más «rígida» a mayores frecuencias, debido a que se acerca a un estado no relajado. Las curvas presentan una inflexión en el rango de frecuencias comprendido entre los 10 y los $100 \mathrm{~Hz}$, que se encuentra directamente asociada al máximo de atenuación localizado en el mismo rango de frecuencias en la Figura 5.3a. A su vez, podemos observar, para el caso $\left\langle S_{w}\right\rangle=70 \%$ (curva azul), una segunda inflexión comienza a manifestarse en el rango de los kHz. Nuevamente, podemos asociar esta inflexión a las características de las curvas de atenuación, es decir, al segundo proceso de relajación localizado en altas frecuencias (Figura 5.3a). Por último, se observa que las velocidades decrecen a medida que la saturación pasa de $30 \%$ a $70 \%$ para todas las frecuencias. En particular, la mayor disminución se concentra en el rango de bajas frecuencias (1 a $10 \mathrm{~Hz}$ ), donde la velocidad decrece $\sim 75 \mathrm{~m} / \mathrm{s}$.

La Figura 5.4b muestra las velocidades sísmicas a estadíos tardíos del proceso de imbibición, correspondientes con el panel superior de la Figura 5.3b, es decir, para saturaciones globales de $\left\langle S_{w}\right\rangle=90 \%,\left\langle S_{w}\right\rangle=94 \%$, y $\left\langle S_{w}\right\rangle=98 \%$. Si analizamos la dispersión de la velocidad para el caso $\left\langle S_{w}\right\rangle=90 \%$ (curva negra) vemos dos inflexiones, una en bajas frecuencias y otra en altas. En este caso, como se observa en el panel 
superior de la Figura 5.3b, el frente no ha atravesado completamente la muestra y, además, existen «patches» de gas y agua detrás del frente, por lo cual podemos presumir la existencia de dos procesos de relajación asociados a estos contrastes de compresibilidad. Este comportamiento puede observarse en la curva de atenuación graficada en la Figura 5.3b (curva negra), donde se observan dos máximos. Sin embargo, una vez que el frente de saturación atraviesa la muestra completamente, tal como se observa para saturaciones $\left\langle S_{w}\right\rangle=94 \%$ y $\left\langle S_{w}\right\rangle=98 \%$, las curvas de velocidad asociadas (curvas rojo claro y rojo oscuro) presentan solamente una inflexión en altas frecuencias. Por último, de manera contraria a lo sucedido en estadíos tempranos del proceso de inyección, observamos un aumento de la velocidad con la saturación global, para todas las frecuencias. El mayor incremento de velocidad se da entre los $10^{4}$ y $10^{5} \mathrm{~Hz}$, donde la velocidad aumenta $\sim 70 \mathrm{~m} / \mathrm{s}$.

Los resultados numéricos evidencian que las distribuciones de saturación resultantes de los experimentos de imbibición producen niveles de dispersión de la velocidad sísmica relativamente bajos. Evidentemente, la precisión experimental al medir variaciones en la velocidad depende, en gran parte, del instrumental utilizado. Lopes et al. (2014) establecen que la precisión en sus mediciones experimentales es del orden de 7 $[\mathrm{m} / \mathrm{s}]$. Esto implica que los niveles de dispersión obtenidos en este ejemplo son experimentalmente medibles en todos los casos, con salvedad de la curva de $\left\langle S_{w}\right\rangle=30 \%$ (Figura 5.4a), cuyos niveles de dispersión son significativamente bajos.

Una de las características más estudiadas de la velocidad sísmica es su relación con la saturación (p. ej., Gassmann, 1951; Lebedev et al., 2009; Monsen y Johnstad, 2005; Alemu et al., 2013). En líneas generales, la variación de $V_{p}$ con $\left\langle S_{w}\right\rangle$ depende tanto de la densidad promedio del agregado $\left\langle\rho_{b}\right\rangle$ como del módulo de onda plana de la roca (ecuación (5.15)). En este sentido, la densidad promedio aumenta con el nivel de saturación $\left\langle S_{w}\right\rangle$, disminuyendo de forma gradual la velocidad $V_{p}$. Sin embargo, para saturaciones altas, el módulo de onda plana aumenta y domina por sobre el efecto de la densidad promedio del agregado. Como consecuencia, $V_{p}$ crece de manera abrupta al acercarse a saturaciones globales cercanas a $100 \%$ (p. ej., Knight y NolenHoeksema, 1990; Domenico, 1974). Este comportamiento se muestra en la Figura 5.4, donde la velocidad disminuye con la saturación en la Figura 5.4a, y luego aumenta para saturaciones cercanas al $100 \%$ en la Figura 5.4b.

El comportamiento de la velocidad con la saturación suele ser modelado por medio de la ecuación de Gassmann (Gassmann, 1951). En particular, el modelo de Gassmann asume que el medio poroso es homogéneo y que se encuentra saturado por un único fluido cuya presión poral está en equilibrio. Por lo cual, esta aproximación puede emplearse para encontrar el comportamiento de la velocidad en el límite de bajas frecuencias. Si la roca posee más de un fluido, el módulo de volumen efectivo del fluido puede estimarse utilizando ley de mezcla de Wood (ecuación (5.17)). En este sentido, la estimación de Gassmann empleando la ley de Wood (GW) responde a (p. ej., Johnson, 2001)

$$
V_{p}^{\mathrm{GW}}=\sqrt{\frac{M_{c}^{\mathrm{GW}}}{\rho_{b}}}
$$

donde $M_{c}^{\mathrm{GW}}=K_{m}+4 / 3 \mu_{m}+\alpha^{2} M$. En esta ecuación, $K_{m}, \mu_{m}, \alpha$, y $M$ son los parámetros efectivos asociados a una roca homogénea saturada con una mezcla uniforme de fases fluidas. 


\subsection{Análisis de la atenuación y dispersión sísmica durante un experimento de}

imbibición

Debido a que en nuestro caso la matriz de la roca de prueba no es homogénea (Figura 5.2b), es necesario calcular las propiedades efectivas equivalentes para emplear el modelo de GW. En este sentido, las magnitudes $\rho_{b}^{\mathrm{eq}}, K_{m}^{\mathrm{eq}}, \mu_{m}^{\mathrm{eq}}, \alpha^{\mathrm{eq}}$, y $M^{\mathrm{eq}}$ pueden estimarse empleando la porosidad media $\langle\phi\rangle$, la saturación global $\left\langle S_{w}\right\rangle$, y las relaciones (5.3) y (5.4). Veamos entonces qué sucede con la relación entre velocidad y saturación durante el experimento de imbibición en relación al límite de GW.

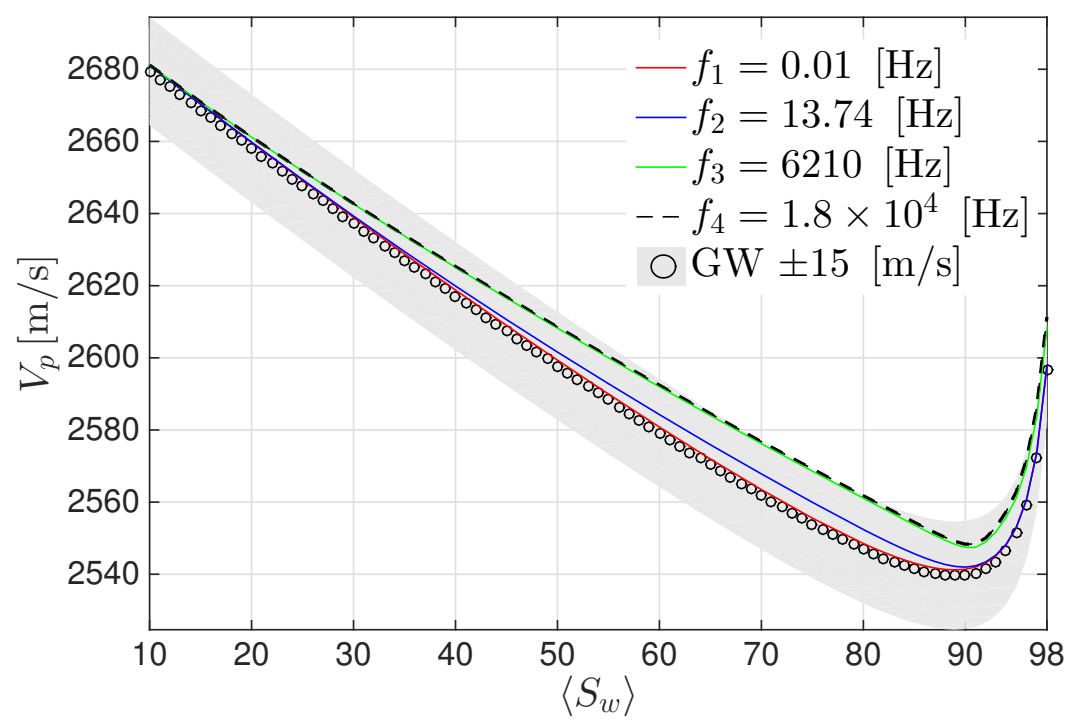

Figura 5.5. Velocidad de fase compresional en función de la saturación. Las curvas muestran el comportamiento de $V_{p}$ con $\left\langle S_{w}\right\rangle$ para cuatro frecuencias diferentes. Se grafica la curva de Gassmann-Wood (GW) para un medio homogéneo con porosidad $\langle\phi\rangle$, como referencia del comportamiento en el límite de bajas frecuencias.

En la Figura 5.5 se observa la variación de la velocidad con la saturación global de agua durante el proceso de imbibición, para diferentes frecuencias, obtenidas con el método de upscaling numérico. La velocidad de GW está representada por una linea discontinua de círculos. Además, se grafica, por medio de un sombreado gris, la zona donde las velocidades se encuentran a $\pm 15[\mathrm{~m} / \mathrm{s}]$ de la curva de GW. En particular, se grafica el comportamiento de la velocidad durante el proceso de imbibición para cuatro frecuencias: Una frecuencia significativamente baja $f_{1}=0.1 \mathrm{~Hz}$, asociada a un régimen relajado; las frecuencias $f_{2}=13.74 \mathrm{~Hz}$ y $f_{3}=6210 \mathrm{~Hz}$, asociadas a los dos máximos de atenuación para $\left\langle S_{w}\right\rangle=70 \%$ (Figura 5.3a); y la frecuencia $f_{4}=1.8 \times 10^{4} \mathrm{~Hz}$, asociada al único máximo de atenuación para $\left\langle S_{w}\right\rangle=98 \%$ (Figura 5.3b). Se observa que el comportamiento de la velocidad con la saturación puede ser modelado relativamente bien por la curva de GW. En el caso particular del comportamiento de la velocidad para las frecuencias $f_{3}$ y $f_{4}$, la variación respecto del límite de $\mathrm{GW}$ es inferior a 15 $[\mathrm{m} / \mathrm{s}]$.

Es importante resaltar que el campo de saturación de una muestra porosa depende de las características del proceso de inyección. En este sentido, los procesos de imbibición y drenaje constituyen dos regímenes extremos caracterizados por curvas de presión capilar y de permeabilidad relativa particulares (p. ej., Soldi et al., 2017). Esto, a su vez, genera un efecto de histéresis, que posee consecuencias significativas para la res- 
puesta sísmica asociada (Yin et al., 1992; Le Ravalec et al., 1996; Zhang et al., 2015). En particular, los experimentos de imbibición están asociados a niveles de atenuación y dispersión menores que los experimentos de drenaje (p. ej., Cadoret et al., 1995, 1998). La tendencia a observar niveles de disipación bajos durante procedimientos de imbibición se debe a que, como inicialmente la muestra contiene altos niveles de saturación de gas, es muy difícil que se generen regiones completamente saturadas con agua. Las regiones que se encuentran completamente saturadas, o altamente saturadas, con agua son cruciales para generar contrastes de compresibilidad dentro de la roca. Esto se debe a que el módulo volumétrico del fluido efectivo está dado por el promedio armónico de los módulos volumétricos individuales (ecuación (5.17)). La Figura 5.6 muestra el comportamiento del módulo volumétrico del fluido efectivo en función de la saturación de agua. Podemos observar que pequeñas cantidades de gas son suficientes como para producir un módulo efectivo muy cercano al del gas. En otras palabras, esto implica que, a menos que existan regiones esencialmente saturadas con agua, los contrastes de compresibilidad entre las diferentes partes de la roca tienden a ser débiles y por lo tanto están asociados a niveles de atenuación y dispersión por flujo inducido por onda en la mesoescala relativamente bajos. Como se observa en la Figura 5.3b, el escenario más dispersivo y atenuante tiene lugar cuando la saturación global de la muestra se encuentra cerca de la saturación máxima de agua, y se debe a que aparecen «patches» con saturaciones muy cercanas a la saturación máxima de agua rodeados de zonas que contienen gas.

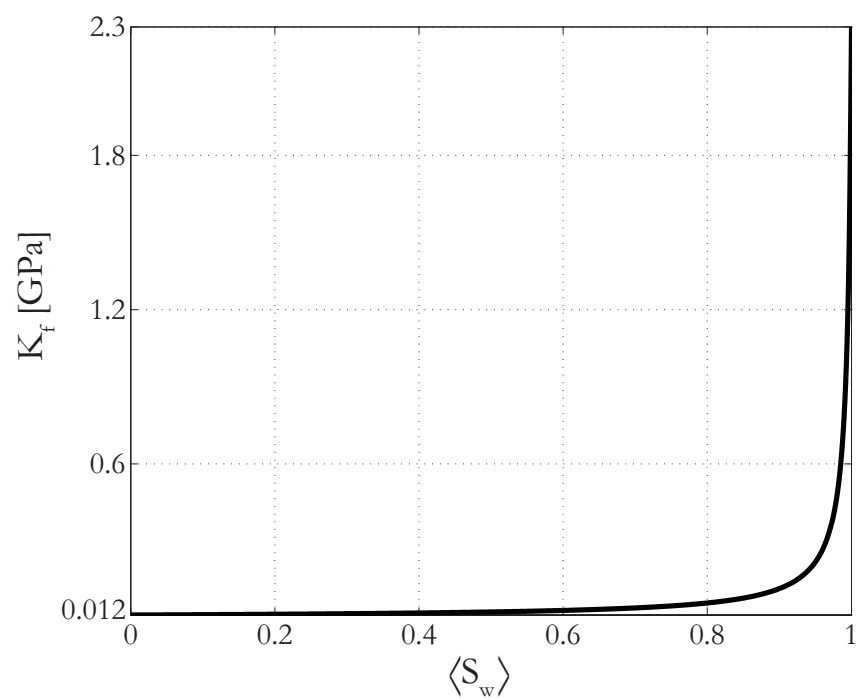

Figura 5.6. Módulo volumétrico del fluido efectivo $K_{f}$ en función de la saturación de agua $S_{w}$. $K_{f}$ varia entre $K_{g}$ y $K_{w}$ para niveles de saturación de agua con valores entre $0 \%$ y $100 \%$, respectivamente. Las propiedades físicas del gas y del agua están dadas en la Tabla 5.1. 


\subsubsection{Interpretación física de las curvas de atenuación y dis- persión}

A continuación analizaremos las razones físicas de los comportamientos observados en las curvas de atenuación y dispersión representadas en las Figuras 5.3 y 5.4. Para ello emplearemos la metodología presentada en el Capítulo 3, que permite calcular la contribución local a la atenuación $q_{i j}^{-1}$. La distribución espacial de $q_{i j}^{-1}$ nos permitirá determinar las regiones que efectivamente contribuyen al valor de atenuación para cada frecuencia.

Para explorar la distribución espacial de $q_{i j}^{-1}$ elegimos los estadíos $\left\langle S_{w}\right\rangle=70 \%$ y $\left\langle S_{w}\right\rangle=98 \%$. El primero es un estadío temprano que evidencia dos máximos de atenuación (ver Figura (5.3)a, curva azul), mientras que el segundo posee un máximo en altas frecuencias de muy alta amplitud (ver Figura (5.3)b, curva roja).

La contribución local a la atenuación para $\left\langle S_{w}\right\rangle=70 \%$, representada en las Figuras $5.7 \mathrm{~b}$ y $5.7 \mathrm{c}$, corresponde a las frecuencias $f_{2}=13.74 \mathrm{~Hz}$ y $f_{3}=6210 \mathrm{~Hz}$, respectivamente. Estas dos frecuencias corresponden a las ubicaciones aproximadas de los dos máximos de atenuación (Figura 5.3a). Para facilitar el análisis, incluimos en la Figura 5.7a la distribución de fluidos dentro de la muestra para este estado global de saturación. Una comparación directa de las Figuras 5.7a y 5.7b permite observar que, en la frecuencia asociada al primer máximo de atenuación $f_{2}$, la disipación ocurre principalmente en la región vecina al frente de saturación caracterizada por altos valores de saturación de agua. Allí existe un cambio abrupto de compresibilidad al pasar de una zona saturada principalmente con agua a una saturada principalmente por gas. En el caso de la frecuencia asociada al segundo máximo de atenuación $f_{3}$, la disipación de la energía tiene lugar detrás del frente de saturación (Figura 5.7c). Una comparación entre las Figuras 5.7a y 5.7c permite observar que estas zonas con altos valores de $q_{i j}^{-1}$ corresponden a los bordes de «patches» caracterizados por altos valores de saturación de agua. Este análisis demuestra que, como fue sugerido anteriormente, el primer máximo de atenuación observado en la Figura 5.3a corresponde al contraste de saturación generado por el frente de saturación, mientras que el segundo máximo está asociado con las regiones que poseen altos valores de saturación de agua y que se encuentran por detrás del frente.

La Figura 5.7e muestra la contribución local a la atenuación para $\left\langle S_{w}\right\rangle=98 \%$ en la frecuencia $f_{4}=1.8 \times 10^{4} \mathrm{~Hz}$, la cual está asociada al máximo de atenuación de la Figura 5.3b. Comparando la Figura 5.7d, donde se muestra la distribución de saturación, y la Figura 5.7e, podemos ver que las regiones donde existe disipación se corresponden con los bordes de los «patches» caracterizados por poseer altos valores de saturación de agua. Además, si comparamos las Figuras 5.7c y 5.7e, observamos que la presencia de valores locales de saturación de agua más altos para $\left\langle S_{w}\right\rangle=98 \%$ generan contrastes de comprensibilidad mayores y, por lo tanto, mayores niveles de disipación local de la energía. Al mismo tiempo, a medida que aumenta la saturación de $\left\langle S_{w}\right\rangle=70 \%$ a $\left\langle S_{w}\right\rangle=98 \%$, la fracción de la roca que contiene regiones con saturaciones locales de agua cercanas a $100 \%$ aumenta, por lo cual también este hecho favorece el aumento de los niveles de atenuación.

Como se ha mencionado en la Sección 2.3 de este trabajo de Tesis, la frecuencia asociada al máximo de atenuación $f_{c}=\omega_{c} / 2 \pi$, se encuentra influenciada por el tamaño 


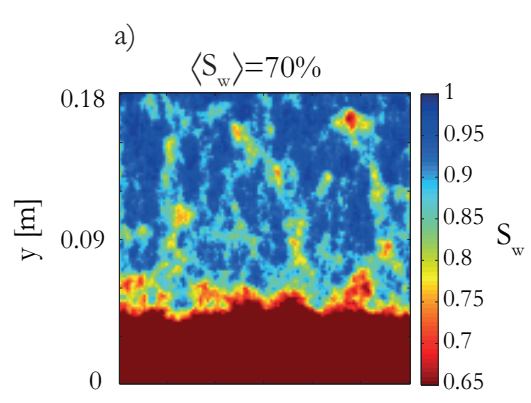

d)

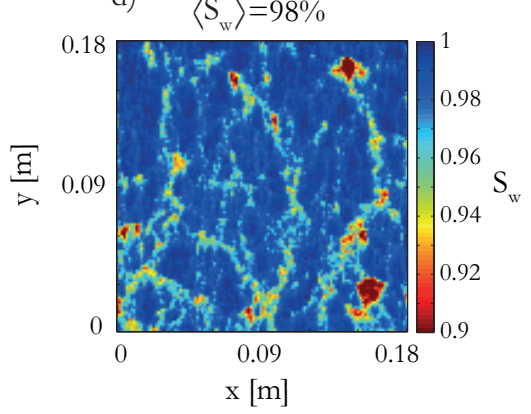

b) b) $f_{2}=13.74[\mathrm{~Hz}]$

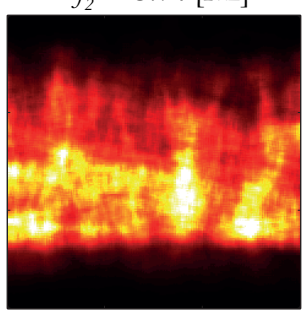

e) $f_{4}=1.8 \times 10^{4}[\mathrm{~Hz}]$

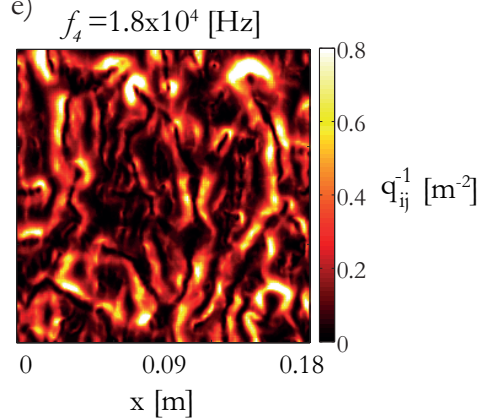

Figura 5.7. Distribución espacial de $q_{i j}^{-1}$ en la muestra sujeta al proceso de imbibición para $\left\langle S_{w}\right\rangle=70 \%$ utilizando la frecuencia del (b) primer y (c) segundo máximo de atenuación y en el caso de $\left\langle S_{w}\right\rangle=98 \%$ utilizando (e) la frecuencia asociada al único máximo de atenuación. Los paneles (a) y (d) muestran la distribución de fluidos dentro de la muestra para $\left\langle S_{w}\right\rangle=70 \%$ y $\left\langle S_{w}\right\rangle=98 \%$, respectivamente.

característico $a_{\text {meso }}$ de las heterogeneidades involucradas en el proceso de relajación de presión poral. Es decir, la atenuación máxima tiene lugar en una frecuencia $f_{c}$ que responde a la ecuación (2.52), por lo cual, $f_{c}$ es inversamente proporcional al tamaño característico de las heterogeneidades $a_{\text {meso }}$ Podemos observar en las Figuras 5.7a y $5.7 \mathrm{~d}$ que

$$
a_{\text {meso }}^{\text {frente }}>a_{\text {meso }}^{\text {patch }}
$$

donde $a_{\text {meso }}^{\text {frente }}$ y $a_{\text {meso }}^{\text {patch }}$ denotan el tamaño característico del frente de saturación y de los «patches» de agua que se encuentran detrás del mismo, respectivamente. Por lo cual, la ecuación $(2.52), \omega_{c} \simeq D / a_{\text {meso }}^{2}$, implica que el máximo de atenuación relacionado con el frente de saturación ocurre a frecuencias menores que el producido por los «patches» aislados, tal como se observa en las Figuras 5.3a y 5.3b.

Una característica de las curvas de atenuación de la Figura 5.3a es el corrimiento del máximo asociado a la presencia del frente de saturación hacia frecuencias menores a medida que se incrementa la saturación global de la muestra. Este comportamiento posee su contraparte en las curvas de dispersión (ver Figura 5.4a), donde, de manera menos notoria, se observa que la primera inflexión se desplaza hacia bajas frecuencias. La ecuación (2.52) permite explicar este corrimiento. Es decir, a medida que el proceso de imbibición tiene lugar, el frente de saturación avanza y la región que contiene niveles locales de saturación de agua altos crece. Por lo tanto, el tamaño característico $a_{\text {meso }}^{\text {frente }}$ aumenta y, consecuentemente, $f_{c}$ decrece. Es importante remarcar que la difusividad $D$ es un parámetro que depende de las propiedades físicas de la matriz y del fluido efectivo. De hecho, este parámetro aumenta a medida que aumenta la saturación, haciéndose particularmente grande para saturaciones cercanas a $S_{w}=100 \%$. De acuerdo con 
(2.52), $f_{c}$ es directamente proporcional a $D$. Entonces, hay una competencia entre los efectos producidos por el tamaño de la heterogeneidad y aquellos relacionados con el valor de la difusividad. Los resultados de la Figuras 5.3a y 5.4a indican que, en el caso del frente de saturación, los cambios en $a_{\text {meso }}$ juegan un papel predominante frente a las variaciones de $D$.

Por otro lado, tanto el máximo presente en la Figura 5.3b como la inflexión en altas frecuencias presente en la Figura 5.4b se encuentran asociados a la presencia de «patches» aislados caracterizados por altos niveles de saturación de agua. Es interesante observar que mientras la saturación de la muestra aumenta de $\left\langle S_{w}\right\rangle=90 \%$ a $98 \%$, la frecuencia característica se desplaza hacia altas frecuencias. Teniendo en cuenta que tanto el tamaño de los «patches» $a_{\text {meso }}^{\text {patch }}$ como los niveles locales de saturación aumentan, el comportamiento presente en la Figura 5.3b indica que, en este caso, el movimiento en frecuencia se encuentra dominado por un aumento local en la difusividad.

Finalmente, podemos emplear la ecuación (2.52) para estimar el tamaño característico de las heterogeneidades $a_{\text {meso }}^{\text {frente }}$ y $a_{\text {meso }}^{\text {patch }}$ utilizando valores aproximados de $f_{c}$ y $D$, en cada caso. Por ejemplo, podemos emplear la ecuación (2.52) para analizar los casos representados en las Figuras 5.7b y 5.7e. En cada caso, seleccionamos la frecuencia asociada al máximo de atenuación de la Figura 5.3, y las propiedades del fluido y la matriz se calculan tomando una porosidad media igual a 0.3 , y un fluido efectivo que depende del grado de saturación de agua de la heterogeneidad. Considerando que el valor promedio de la saturación de agua de la región donde ocurre la disipación de la energía en la situación contemplada en la Figura 5.7b es de aproximadamente $80 \%$, obtenemos un tamaño característico $a_{\text {meso }}^{\text {frente }} \simeq 6 \mathrm{~cm}$. Es interesante notar que este tamaño es comparable con el espesor de la región responsable de la atenuación (Figura $5.7 b)$. De igual manera, considerando una saturación de agua igual a $99 \%$ para los «patches» que controlan la disipación de la energía en la Figura 5.7e, obtenemos un tamaño característico $a_{\text {meso }}^{\text {patch }} \simeq 0.5 \mathrm{~cm}$. Comparando las Figuras $5.7 \mathrm{~d}$ y $5.7 \mathrm{e}$, observamos que también en este caso el tamaño característico es representativo de los «patches» saturados con agua que contribuyen efectivamente a la atenuación total de la muestra.

\subsection{Efecto de la tasa de inyección en la atenuación y dispersión sísmica}

El régimen de flujo de un proceso de imbibición está determinado por el balance entre las fuerzas viscosas, gravitacionales, y capilares (Lopes et al., 2014; Riaz et al., 2007). La tasa de inyección es un parámetro clave ya que afecta la relación entre estas fuerzas y, por lo tanto, determina en parte la evolución del campo de saturación dentro del medio poroso (Helmig et al., 2006). A continuación, exploraremos los efectos de cambiar la tasa de inyección sobre las características de la distribución de fluidos y sobre la atenuación y dispersión sísmica asociada. Con este objetivo, repetiremos el análisis presentado en las Secciones previas, pero consideraremos tres tasas de inyección diferentes: $q_{w_{1}}^{T}=$ $1 \times 10^{-4}[\mathrm{~m} / \mathrm{s}], q_{w_{2}}^{T}=5 \times 10^{-4}[\mathrm{~m} / \mathrm{s}], q_{w_{3}}^{T}=1 \times 10^{-3}[\mathrm{~m} / \mathrm{s}]$. Estas se encuentran en el rango de velocidades de inyección usualmente empleado en experimentos de laboratorio ( $\mathrm{p}$. ej., Iglauer et al., 2011; Lebedev et al., 2014; Nakagawa et al., 2013). Consideraremos las mismas propiedades de roca y fluidos que hemos utilizados en las secciones anteriores. 

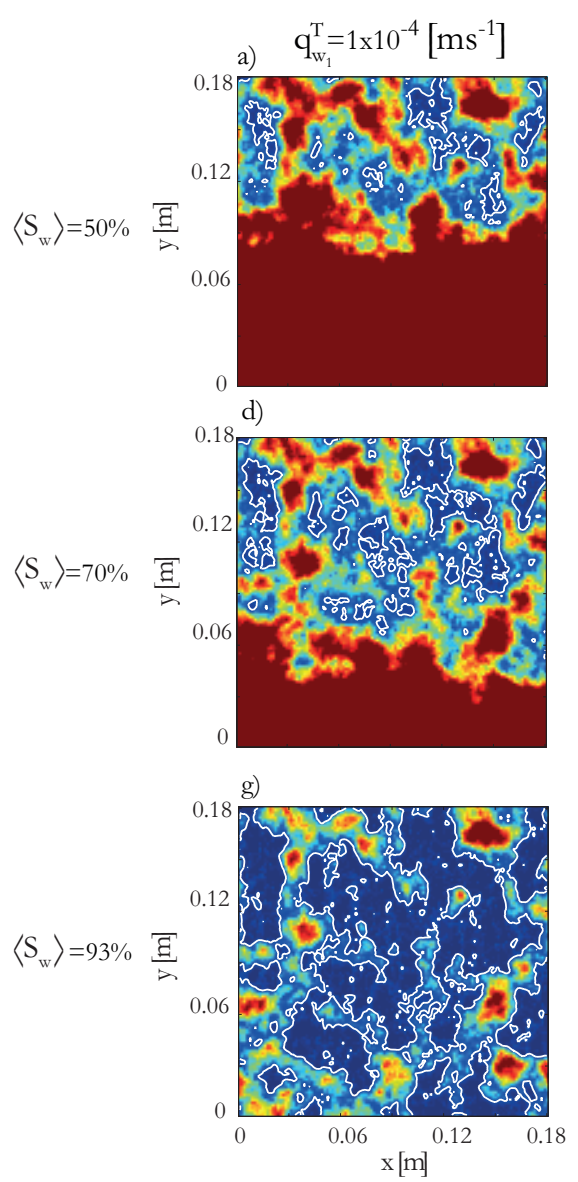

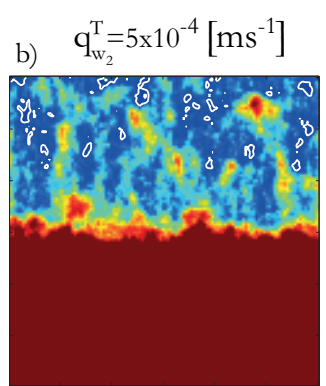

e)

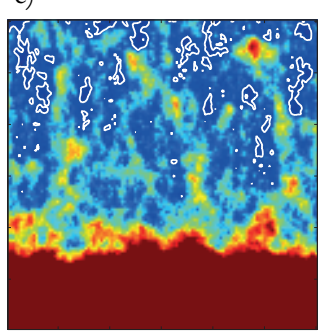

h)

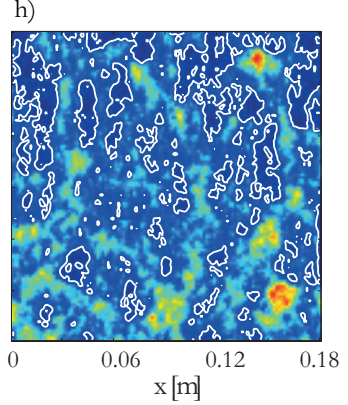

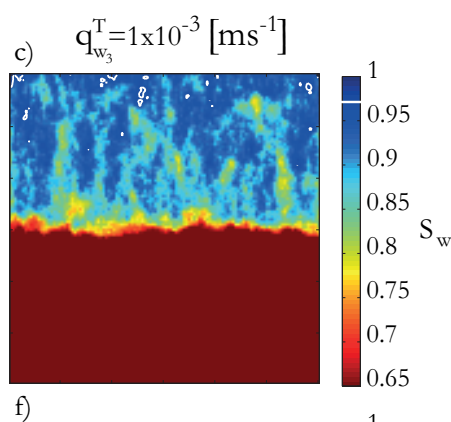
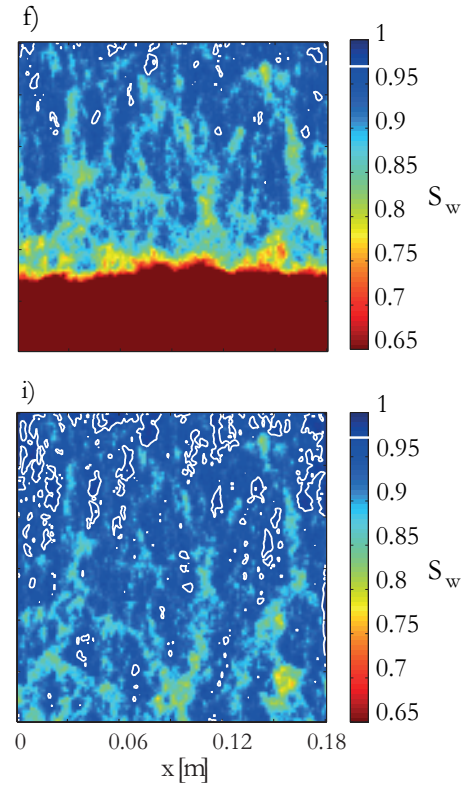

Figura 5.8. Campos de saturación de agua resultantes de experimentos de imbibición con tasas de inyección de (a, d, g) $q_{w_{1}}^{T}=1 \times 10^{-4}[\mathrm{~m} / \mathrm{s}],(\mathrm{b}, \mathrm{e}, \mathrm{h})$ $q_{w_{2}}^{T}=5 \times 10^{-4}[\mathrm{~m} / \mathrm{s}]$, y $(\mathrm{c}, \mathrm{f}, \mathrm{i}) q_{w_{3}}^{T}=1 \times 10^{-3}[\mathrm{~m} / \mathrm{s}]$. Las diferentes filas corresponden a diferentes instantes del proceso de imbibición caracterizadas por niveles de saturación de $\left\langle S_{w}\right\rangle=50 \%$ (a, b, c),$\left\langle S_{w}\right\rangle=70 \%$ (d, e, f) , y $\left\langle S_{w}\right\rangle=93 \%$ (g, h, i). Las lineas de contorno blancas delimitan las regiones donde la saturación local de agua supera el $97 \%$.

La Figura 5.8 muestra los campos de saturación resultantes de los tres experimentos de imbibición. Cada columna muestra los resultados de una tasa de inyección diferente, aumentando de izquierda a derecha. Por otro lado, cada fila corresponde a una misma saturación global de la muestra, alcanzada a tiempos diferentes, aumentando de arriba hacia abajo. Es importante resaltar que cada proceso de imbibición posee una saturación global máxima diferente, asociada con el estado estacionario alcanzado. Dada la importancia de las regiones con valores locales de saturación de agua altos para producir flujo inducido por onda, se incluye en cada panel una línea de contorno que delimita las regiones donde la saturación local de agua se encuentra por arriba de $97 \%$. Esto corresponde a un módulo volumétrico de $0.35 \mathrm{GPa}$ para el fluido efectivo, como puede verse en la Figura 5.6.

Observamos en la Figura 5.8 que, a medida que la tasa de inyección aumenta, los campos de saturación cambian de un proceso de inyección con un entrampamiento sustancial de gas y agua caracterizado por un frente de saturación irregular (Figuras 
5.8a, 5.8d, 5.8g) a un proceso cuya principal característica es la presencia de un frente de saturación abrupto (Figuras 5.8c, 5.8f, 5.8i). Es decir, cuando el proceso de imbibición posee una tasa de inyección baja, los gradientes de presión de la fase mojante son débiles y la acción de las fuerzas capilares es más notoria. Por lo tanto, la fase mojante tiende a ingresar por capilaridad en las zonas de menor permeabilidad, saturándolas. Consecuentemente, el proceso de imbibición presenta un frente de saturación dendrítico. En este escenario, se suelen formar regiones aisladas con alta saturación de agua detrás del frente de saturación, como se observa en las Figuras 5.8a, 5.8d, y 5.8g. Por otro lado, cuando el proceso de imbibición posee una tasa de inyección alta, el gradiente macroscópico de presión de agua genera una rápida saturación de todas las regiones de la muestra de roca, y los efectos de la capilaridad son casi imperceptibles. De manera correspondiente, la fase mojante avanza presentando un frente de saturación regular, del tipo «pistón», como se observa en las Figuras 5.8c, 5.8f, y 5.8i. En ellas, puede observarse que las regiones con valores altos de saturación de agua detrás del frente están relativamente ausentes y tienden a aparecer únicamente para valores de saturación global altos.

Evidentemente, las ondas sísmicas responden de manera diferente a la evolución de cada proceso de imbibición. Para explorar la atenuación en estos escenarios, incluimos en la Figura 5.9 la atenuación de onda $\mathrm{P}$ como función de la saturación global y la frecuencia para las tres tasas de inyección empleadas en la Figura 5.8. Observamos que, para bajas tasas de inyección, el máximo de atenuación de bajas frecuencias localizado entre los $10 \mathrm{~Hz}$ y los $100 \mathrm{~Hz}$, asociado con el frente de saturación, es insignificante (Figura 5.9a). Esta ausencia de disipación en las cercanías del frente para tasas de inyección bajas se debe a que el frente es altamente irregular, por lo cual, los procesos de relajación de presión que tienen lugar en el frente interactúan entre sí y tienden a cancelarse. En cambio, el máximo de atenuación generado por los «patches» ubicados detrás del frente de saturación es relativamente alto, con valores de $Q_{p}^{-1}$ mayores a 0.01 para valores de $\left\langle S_{w}\right\rangle$ por encima de $90 \%$. La importancia de esta manifestación de flujo inducido por onda se debe a que las tasas de inyección bajas favorecen la presencia de regiones con altos valores de saturación de agua detrás del frente de saturación, lo cual crea contrastes de compresibilidades significativos con el entorno.

Cuando aumentamos la tasa de inyección, la atenuación experimenta cambios significativos, como se puede ver en las Figuras 5.9b y 5.9c. Observamos la aparición de un máximo de atenuación localizado aproximadamente en $14 \mathrm{~Hz}$ para saturaciones globales de $30 \%$ a $80 \%$, cuya magnitud aumenta para tasas de inyección crecientes. Notamos en las Figuras $5.9 \mathrm{~b}$ y $5.9 \mathrm{c}$ que este máximo de bajas frecuencias presenta un comportamiento transitorio. Es decir, aumenta a medida que el tamaño característico del frente de saturación y la saturación de la muestra crecen, y, finalmente, desaparece una vez que el frente de saturación abandona la muestra. Además, como una tasa de inyección alta no favorece la generación de zonas aisladas de alta saturación de agua detrás del frente, el máximo de atenuación localizado aproximadamente en $1.8 \times 10^{5} \mathrm{~Hz}$ tiende a reducirse con el aumento de la tasa de inyección, como se observa en las Figuras 5.9b y $5.9 \mathrm{c}$.

A continuación analizaremos el efecto de la tasa de inyección en el comportamiento de las curvas de velocidad. Para ello, graficamos la velocidad en función de la frecuencia, para diferentes estadíos del proceso de imbibición, y diferentes tasas de inyección. 
a)

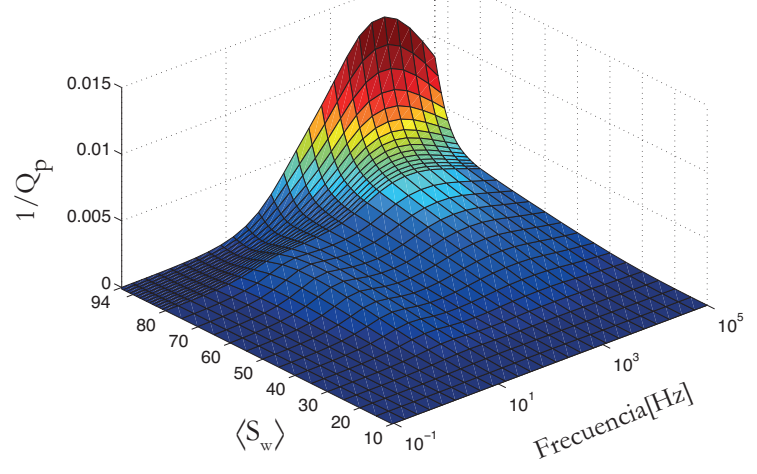

b)

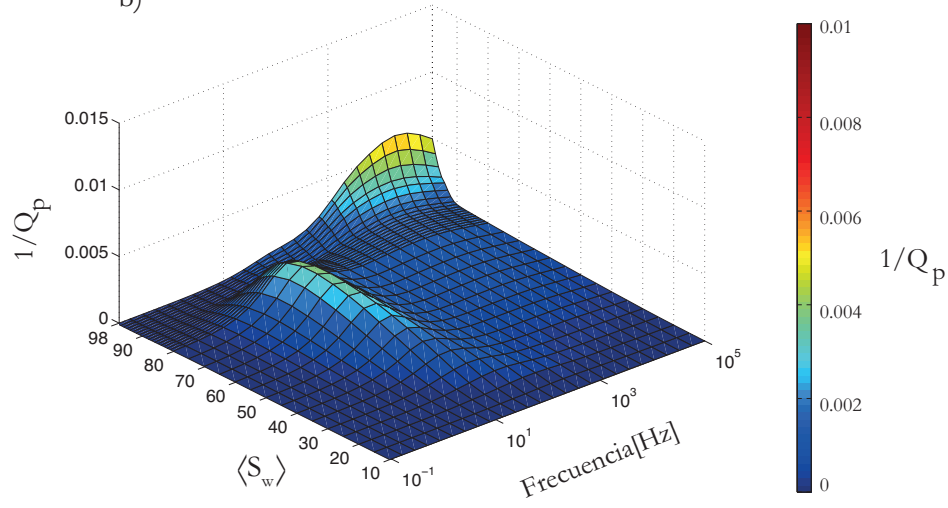

c)

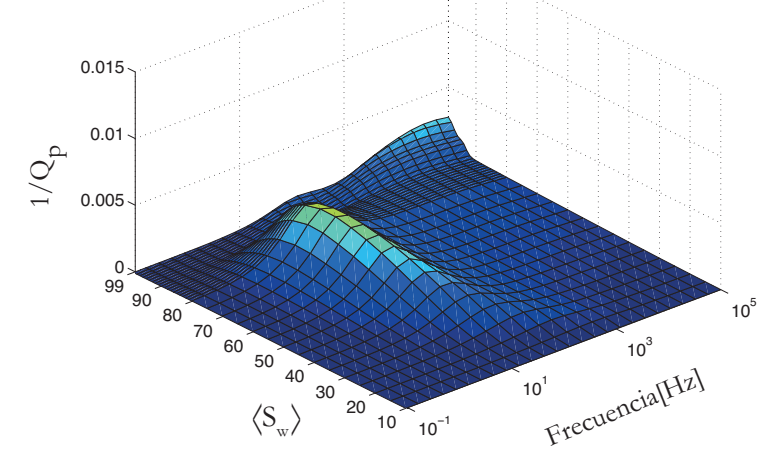

Figura 5.9. Inverso del factor de calidad $1 / Q_{p}$ en función de la saturación global y la frecuencia para las distribuciones de fluidos obtenidas con tasas de inyección (a) $q_{w_{1}}^{T}=1 \times 10^{-4}[\mathrm{~m} / \mathrm{s}],(\mathrm{b}) q_{w_{2}}^{T}=5 \times 10^{-4}[\mathrm{~m} / \mathrm{s}]$, y $(\mathrm{c}) q_{w_{3}}^{T}=1 \times 10^{-3}[\mathrm{~m} / \mathrm{s}]$.

La Figura 5.10 muestra la velocidad de onda $\mathrm{P}$ en función de la frecuencia para estadíos tempranos del proceso de imbibición. Se emplean líneas sólidas de color negro, azul, y rojo al graficar las curvas de dispersión para $q_{w_{1}}^{T}=1 \times 10^{-4}[\mathrm{~m} / \mathrm{s}], q_{w_{2}}^{T}=5 \times 10^{-4}[\mathrm{~m} / \mathrm{s}]$, y $q_{w_{3}}^{T}=1 \times 10^{-3}[\mathrm{~m} / \mathrm{s}]$, respectivamente. Observamos que, en todos los casos considerados en esta figura, la dispersión de la velocidad es despreciable para todas las tasas de inyección seleccionadas. De hecho, si consideramos que el error de medición de laboratorio es aproximadamente $7[\mathrm{~m} / \mathrm{s}]$, el comportamiento de la velocidad puede ser relativamente bien modelado utilizando una aproximación de GW de medio homogé- 

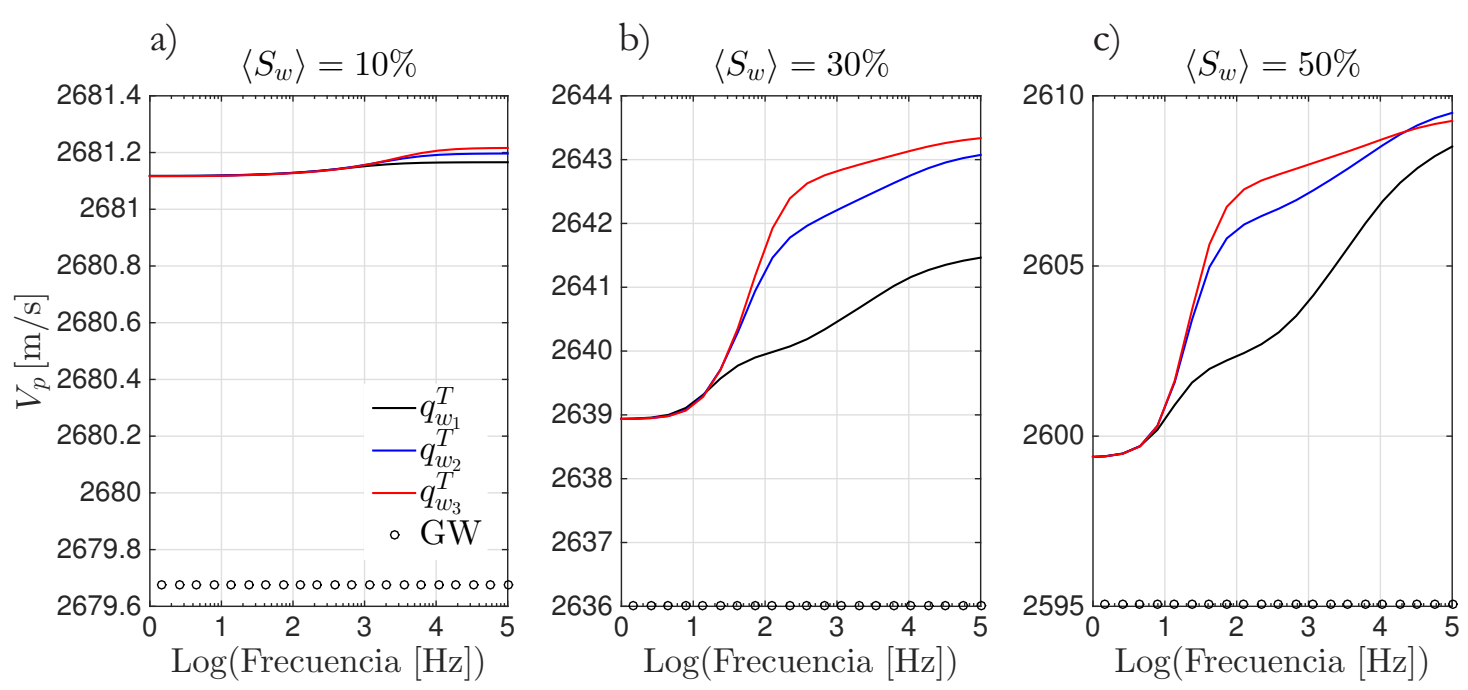

Figura 5.10. Velocidad de fase de onda $\mathrm{P}$ en función de la frecuencia para las diferentes tasas de inyección consideradas en este análisis: $q_{w_{1}}^{T}=1 \times 10^{-4}[\mathrm{~m} / \mathrm{s}]$, $q_{w_{2}}^{T}=5 \times 10^{-4}[\mathrm{~m} / \mathrm{s}]$, y $q_{w_{3}}^{T}=1 \times 10^{-3}[\mathrm{~m} / \mathrm{s}]$. Se ilustran estadíos tempranos del proceso de imbibición.

neo (círculos negros), es decir, empleando la ecuación (5.20). Más allá de los niveles de variación de la velocidad con la frecuencia, la dispersión en las Figuras 5.10a y 5.10b suele ser mayor para tasas de inyección altas (lineas rojas), que para tasas medias (lineas azules) y bajas (lineas negras). Esto se debe a que, para estadíos tempranos del proceso de inyección, la disipación tiene lugar principalmente en las cercanías del frente de saturación, y tiende a ser más significativa para altas tasas. A su vez, observamos que, a medida que la roca se satura, la frecuencia donde tiene lugar la primera inflexión disminuye, debido a un incremento en $a_{\text {meso }}^{\text {frente }}$ Por otro lado, en la Figura 5.10c se comienza a observar una segunda inflexión, localizada en altas frecuencias, asociada a la formación de «patches» de agua detrás del frente. Como hemos observado previamente, las tasas de inyección altas impiden la generación de «patches» de agua con altas saturaciones locales y, por lo tanto, esta segunda inflexión es más pronunciada para tasas de inyección bajas e intermedias que para tasas altas.

En la Figura 5.11 observamos la velocidad en función de la frecuencia para estadíos tardíos del proceso de imbibición, considerando las tres tasas de inyección analizadas. En el caso de las Figuras 5.11a y 5.11b, podemos observar las dos inflexiones, asociadas a la presencia del frente de saturación y a los «patches» aislados detrás del frente. A medida que la saturación global aumenta de $\left\langle S_{w}\right\rangle=70 \%$ a $\left\langle S_{w}\right\rangle=94 \%$, la curva de velocidad asociada a la tasa de inyección baja $q_{w_{1}}^{T}$ sobrepasa a aquellas relacionadas con tasas medias y altas. Sin embargo, al analizar la Figura 5.11b, observamos que, dependiendo del rango de frecuencias analizado, la relación entre $V_{p}$ y $q_{w}^{T}$ puede sufrir «inversiones». Por ejemplo, en la Figura 5.11b, en el rango de frecuencias entre $10 \mathrm{y}$ $100 \mathrm{~Hz}$, la menor velocidad corresponde a la menor tasa de inyección. Luego, en un rango de frecuencias comprendido entre los $100 \mathrm{~Hz}$ y los $5 \mathrm{kHz}$, la menor velocidad está dada por la tasa de inyección media $q_{w_{2}}^{T}$. Finalmente, para frecuencias mayores a $5 \mathrm{kHz}$, la menor velocidad está dada por la mayor tasa de inyección $q_{w_{3}}^{T}$. Este análisis muestra que establecer una relación unívoca entre velocidad y tasa de inyección durante un proceso 

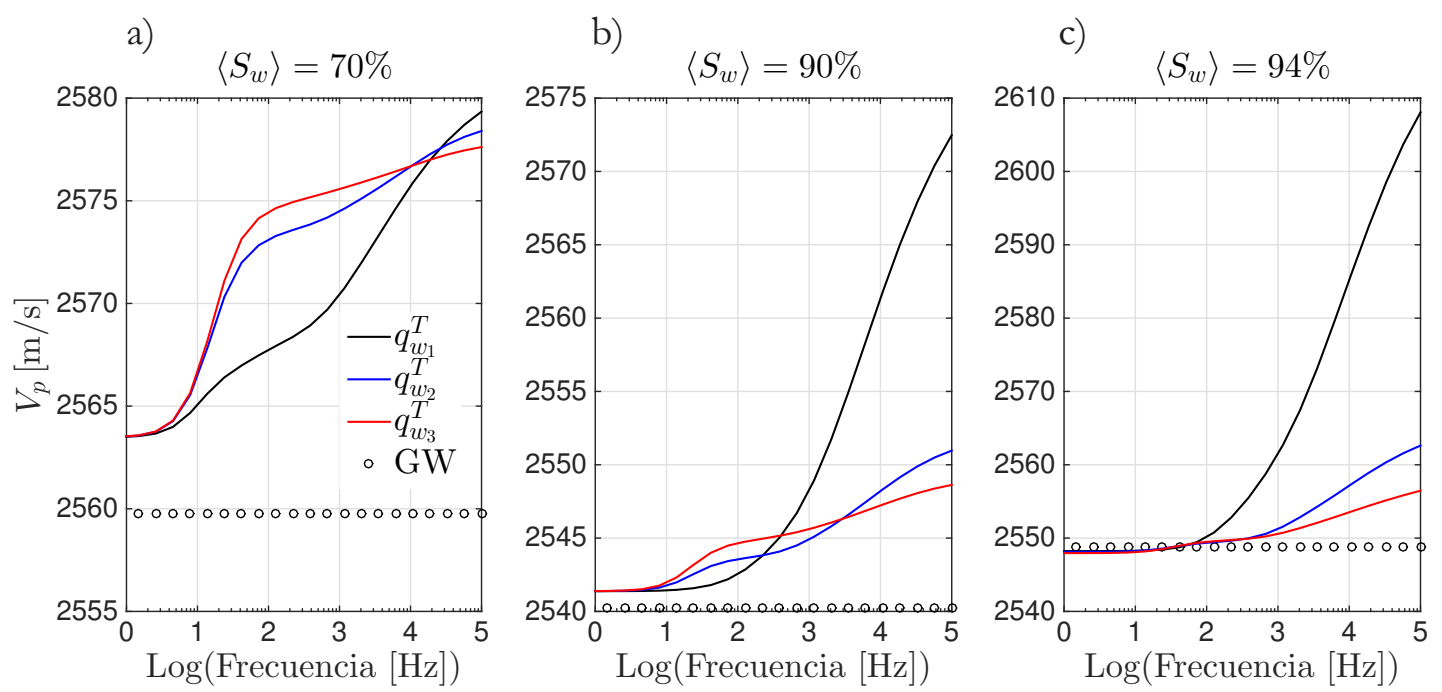

Figura 5.11. Velocidad de fase de onda $\mathrm{P}$ en función de la frecuencia para las diferentes tasas de inyección consideradas en este análisis: $q_{w_{1}}^{T}=1 \times 10^{-4}[\mathrm{~m} / \mathrm{s}]$, $q_{w_{2}}^{T}=5 \times 10^{-4}[\mathrm{~m} / \mathrm{s}]$, y $q_{w_{3}}^{T}=1 \times 10^{-3}[\mathrm{~m} / \mathrm{s}]$. Se ilustran estadíos tardíos del proceso de imbibición.

transitorio es algo difícil. Más allá de esto, para saturaciones relativamente altas (Figura 5.11c) la dispersión generada por tasas de inyección bajas es predominante. En todos los casos contemplados en la Figura 5.11, la dispersión de la velocidad se encuentra por encima del límite observacional. Además, observamos que la curva de GW no constituye una buena aproximación para frecuencias mayores a $1 \mathrm{kHz}$.

\subsection{Discusión de los resultados}

En general, el objetivo de los estudios desarrollados en el contexto de flujo inducido por onda yace en encontrar vínculos entre las propiedades de la roca, la distribución de los fluidos o características de otras heterogeneidades, y las propiedades del dato sísmico. En particular, varios estudios de laboratorio se abocaron a comprender las causas de la disipación sísmica observada durante procesos de inyección de fluidos en muestras de roca (p. ej., Yin et al., 1992; Cadoret et al., 1998). Este objetivo es de significativa importancia, ya que, si comprendemos cómo cambian las firmas sísmicas durante un proceso de inyección de fluidos, podremos utilizar los métodos sísmicos para realizar una caracterización hidráulica del subsuelo.

Nuestro análisis numérico indica que los patrones heterogéneos de saturación producidos durante procesos de imbibición, relacionados con heterogeneidades dentro de las muestras de roca, pueden generar niveles medibles de atenuación y dispersión sísmica debido a flujo inducido por onda en la mesoescala. En particular, podemos observar en las Figuras 5.9 y 5.11 que este mecanismo de disipación se vuelve significativo para saturaciones globales mayores a $90 \%$. Este resultado se corresponde con mediciones de atenuación sísmica realizadas en laboratorio (p. ej., Winkler y Nur, 1979; Murphy, 1984; Yin et al., 1992; Tisato y Quintal, 2013). Por ejemplo, Yin et al. (1992) realizaron experimentos de imbibición en una muestra de arenisca Berea y observaron un máximo 
en las curvas de atenuación en función de la saturación para una saturación global de agua igual $\mathrm{a} \sim 97 \%$. Estos autores atribuyeron el comportamiento de la atenuación con la frecuencia a flujo inducido por onda entre «patches» aislados, generados durante el proceso de imbibición, y su entorno. Recientemente, Tisato y Quintal (2013) estudiaron la atenuación sísmica en una muestra de arenisca Berea para valores de saturación global crecientes. Estos autores observaron atenuación dependiente de la frecuencia para saturaciones globales con valores entre $86.5 \%$ y $97.1 \%$, y argumentaron que se debía a flujo inducido por onda en la mesoescala debido a una distribución heterogénea de los fluidos.

En cuanto a las características de la velocidad sísmica observadas en laboratorio, la mayoría de las mediciones de $V_{p}$ en función de $\left\langle S_{w}\right\rangle$ realizadas en areniscas saturadas, empleando frecuencias de $1 \mathrm{~Hz}$ a $2500 \mathrm{~Hz}$, evidencian una disminución de la velocidad con la saturación seguida de un incremente abrupto de las velocidades para saturaciones mayores a $90 \%$ (p. ej., Batzle et al., 2006; Mikhaltsevitch et al., 2016). Este resultado se encuentra en acuerdo con lo observado en nuestras simulaciones (ver, p ej., Figura 5.5). Batzle et al. (2006) estudió el comportamiento de una arenisca del Mar del Norte para diferentes frecuencias y observó una variación de la velocidad de $\sim 600 \mathrm{~m} / \mathrm{s}$ entre los $5 \mathrm{~Hz}$ y los $5 \times 10^{5} \mathrm{~Hz}$, para una saturación global de $\left\langle S_{w}\right\rangle=90 \%$. Nuestras simulaciones presentan una dispersión de, como máximo, $60 \mathrm{~m} / \mathrm{s}$ de variación en este rango de frecuencias. Por lo cual, podemos asegurar que los niveles de dispersión modelados en este Capítulo son bajos. Sin embargo, es importante resaltar que en gran parte de los estudios citados calculan la atenuación y la dispersión en areniscas cuyas propiedades difieren de la muestra numérica analizada en este Capítulo. Asimismo, ninguno de estos experimentos realiza la saturación de la muestra controlando la tasa de inyección. Esto es problemático a la hora de realizar comparaciones con modelos numéricos o entre diferentes experimentos de laboratorio ya que, como hemos demostrado, la tasa de inyección seleccionada afecta la respuesta sísmica de la roca. Por último, existen mecanismos adicionales de disipación, no modelados en este trabajo, tales como, flujo inducido por onda a escala poral o anaelasticidad de la matriz, cuyos efectos pueden ser considerables durante experimentos de imbibición.

Debido a limitaciones computacionales, el análisis presentado en este Capítulo ha sido realizado utilizando muestras $2-\mathrm{D}$. Sin embargo, se espera que la naturaleza general de los resultados obtenidos se mantenga en muestras 3-D. Además, es importante remarcar que los comportamientos derivados del procedimiento numérico de homogeneización empleado, descripto en el Sección 3.2 de esta Tesis, pueden ser considerados comparables con aquellos obtenidos en el laboratorio utilizando técnicas de oscilación subresonantes (p. ej., Tisato y Madonna, 2012). En este sentido, el máximo de atenuación asociado al frente de saturación y la correspondiente inflexión en la curva de dispersión no podrán ser observados experimentalmente si la atenuación sísmica se obtiene a través de la transmisión de un impulso sísmico. De hecho, en este caso, el frente de saturación será interpretado en los datos como una interfase en la cual las ondas se reflejan. En cambio, los «patches» que contienen niveles altos de saturación de agua, localizados detrás del frente de saturación, sí pueden producir disipación por flujo inducido por onda a escala mesoscópica. Sin embargo, para frecuencias suficientemente altas, en vez de producir atenuación y dispersión debido a flujo inducido por onda, generarán «scattering». Además, a pesar de que los análisis numéricos incluidos 
en este trabajo consideran distribuciones heterogéneas de las propiedades de la matriz, se espera que los resultados encontrados sean aplicables a procedimientos de imbibición realizados en rocas comúnmente consideradas como homogéneas. Esto se debe a que la permeabilidad es un parámetro altamente fluctuante, cuya variación en cortas distancias puede llegar a varios ordenes de magnitud, y cuya determinación en un medio poroso es difícil de lograr. De hecho, los valores de permeabilidad obtenidos de mediciones experimentales son valores efectivos calculados a escala de muestra, y no proveen información sobre las posibles fluctuaciones de permeabilidad que ocurren dentro de las rocas analizadas. Teniendo en cuenta que la permeabilidad es un parámetro clave en los procesos de flujo, se espera que en el laboratorio se generen heterogeneidades en la distribución de los fluidos porales cuyas características sean similares a las observadas en nuestros experimentos numéricos.

Los resultados de las simulaciones numéricas muestran que, más allá del valor de la saturación global, las características geométricas de la distribución de fluidos controlan la respuesta sísmica de la muestra. Como se observa en la Figura 5.8, distintas tasas de inyección generan diferentes distribuciones de gas y agua para la misma saturación global. El impacto de la tasa de inyección en la generación de «patches» de fluidos puede ser analizada a través del número capilar $C_{a}$. Este número adimensional se utiliza para cuantificar la relación existente entre las fuerzas viscosas y capilares. Existen diferentes definiciones para el número capilar, sin embargo, para un proceso de flujo a escalas mesoscópicas este puede ser expresado de acuerdo a (Hilfer y Øren, 1996),

$$
C_{a}=\frac{\eta_{w}\left\langle q_{w}\right\rangle\langle l\rangle}{\langle\kappa\rangle\left\langle p_{d}\right\rangle}
$$

donde $\langle l\rangle,\left\langle q_{w}\right\rangle,\left\langle p_{d}\right\rangle, \mathrm{y}\langle\kappa\rangle$ son valores característicos para la longitud de la muestra, la velocidad de Darcy de la fase mojante, la presión de entrada, y la permeabilidad de la roca, respectivamente. Si un proceso de inyección posee un número capilar $C_{a}$ mayor a 1, las fuerzas viscosas dominan el desplazamiento de los fluidos. De manera contraria, si $C_{a}<1$, las fuerzas capilares controlan las características del mismo (Armstrong et al., 2014). Tomaremos $\langle l\rangle$ como la longitud de la submuestra, $\left\langle q_{w}\right\rangle$ como la tasa de inyección, y $\langle\kappa\rangle$ y $\left\langle p_{d}\right\rangle$ como las medias aritméticas de la permeabilidad y la presión de entrada calculadas en la submuestra, respectivamente. Obtenemos así tres números capilares, $C_{a_{1}}=0.45, C_{a_{2}}=2.24$, y $C_{a_{3}}=4.45$, asociados a las tasas de inyección previamente propuestas, $q_{w_{1}}^{T}=1 \times 10^{-4}[\mathrm{~m} / \mathrm{s}], q_{w_{2}}^{T}=5 \times 10^{-4}[\mathrm{~m} / \mathrm{s}], y$ $q_{w_{3}}^{T}=1 \times 10^{-3}[\mathrm{~m} / \mathrm{s}]$, respectivamente. Entonces, podemos explorar las características de las curvas de atenuación y dispersión en función del número capilar. Los resultados muestran que los procesos de imbibición dominados por procesos viscosos $\left(C_{a}>1\right)$ se caracterizan por una discontinuidad fuerte asociada al frente de saturación (Figuras 5.8c, 5.8f, 5.8i) y, por lo tanto, presentan un máximo de atenuación dominante localizado en frecuencias relativamente bajas. Este se desplaza hacia frecuencias menores a medida que el frente de saturación avanza dentro de la muestra, y luego desaparece cuando el frente abandona la muestra de roca (Figura 5.9c). De manera análoga, las curvas de dispersión de velocidad evidencian una primera inflexión que se desplaza hacia bajas frecuencias y desaparece para muy altas saturaciones globales (Figuras $5.10 \mathrm{y}$ 5.11). Esta manifestación de flujo inducido por onda en la mesoescala tiene un carácter transitorio, asociado a la presencia del frente de saturación dentro de la muestra. Por 
otro lado, en el caso de procesos dominados por fuerzas capilares, los campos de saturación se caracterizan por un alto nivel de entrampamiento de agua y gas y un frente de saturación irregular (Figuras 5.8a, 5.8d, 5.8g). La atenuación y dispersión sísmica se deben principalmente a flujo inducido por onda entre los «patches» con alta saturación de agua y su entorno. Nuestro análisis numérico indica que el máximo de atenuación se localiza en frecuencias relativamente altas y que este se desplaza hacia frecuencias mayores a medida que aumenta la saturación global de la muestra (Figura 5.9a). Con respecto a la dispersión de velocidad, se observa una inflexión en altas frecuencias que se desplaza hacia frecuencias mayores para valores crecientes de saturación global. Estos comportamientos contrastantes, evidenciados en la ubicación y el desplazamiento del máximo de atenuación y del punto de inflexión de las curvas de velocidad hacia altas o bajas frecuencias, indican que puede ser posible discernir si un determinado proceso de imbibición está dominado por fuerzas capilares o viscosas observando la evolución de las firmas sísmicas asociadas.

\subsection{Conclusiones}

Nuestros resultados muestran que los procesos de imbibición pueden generar atenuación y dispersión medible debido a flujo inducido por onda en la mesoescala, especialmente para niveles altos de saturación global. Además, pueden observarse dos manifestaciones de flujo inducido. Un análisis de la contribución local a la atenuación global permite demostrar que la primera manifestación se produce por la existencia de un contraste de compresibilidad asociado al frente de saturación. Por ello, su efecto se desvanece cuando el frente de saturación abandona la muestra. Además, este mecanismo tiende a aparecer cuando el flujo está dominado por fuerzas viscosas, las cuales resultan de utilizar altas tasas de inyección. Por otro lado, la segunda manifestación de flujo inducido por onda afecta a las curvas de atenuación y dispersión en frecuencias más altas, y se produce por la presencia de «patches» caracterizados por alta saturación de agua y que se encuentran localizados detrás del frente de saturación. Esta manifestación de flujo inducido por onda posee un rol importante durante procesos dominados por fuerzas capilares, es decir, para tasas de inyección relativamente bajas. La magnitud de los máximos de atenuación asociados, y de la dispersión correspondiente, tienden a aumentar con la saturación global. Además, mientras el máximo de atenuación y la inflexión en las curvas de velocidad producidos por el frente de saturación se mueve hacia frecuencias menores para saturaciones globales crecientes, los efectos asociados a la presencia de «patches» de alta saturación de agua se desplazan hacia frecuencias mayores. Los resultados de este análisis, en combinación con el procedimiento numérico empleado, constituyen un estudio de gran importancia en el contexto de la interpretación de la atenuación y dispersión sísmica en medios parcialmente saturados en general y durante experimentos de imbibición en particular. 


\section{Capítulo 6}

\section{Atenuación y dispersión en medios parcialmente saturados: Modelos rea- listas vs. modelos binarios de satura- ción}

Al modelar los efectos del flujo mesoscópico en la respuesta sísmica de rocas parcialmente saturadas, se suele asumir que el campo de saturación es binario. Es decir, se considera que los fluidos porales se distribuyen en regiones de tamaños mesoscópicos completamente saturadas por una de las fases, rodeadas por zonas completamente saturadas por la fase restante. Sin embargo, los estudios experimentales evidencian que los campos de saturación están relacionados con las heterogeneidades de la matriz, y que sus valores locales varían de forma continua entre 0 y 1 . Como se ha observado previamente, las curvas de atenuación y dispersión dependen de la distribución de fluidos empleada y, por ello, los modelos binarios de saturación pueden resultar en estimaciones erróneas de la atenuación y velocidad de las ondas. En este Capítulo se analizan las consecuencias de modelar la respuesta sísmica de medios parcialmente saturados empleando campos binarios de saturación. En primer lugar, se analiza la capacidad del modelo de White para replicar las curvas de atenuación y dispersión asociadas a la presencia de un frente de saturación resultante de un proceso de imbibición en una roca homogénea. Luego, se exploran las consecuencias de considerar campos binarios de saturación para representar la respuesta sísmica de una roca heterogénea 2-D sometida a un proceso de imbibición.

\subsection{Introducción}

Desde los trabajos pioneros de White (1975) y White et al. (1975), donde se consideran distribuciones binarias de saturación en medios porosos homogéneos, los campos binarios de saturación han sido ampliamente utilizados en el contexto del flujo inducido por ondas sísmicas a escalas mesoscópicas. En particular, en las últimas dos décadas, los modelos binarios han sido considerados en trabajos de gran impacto en la literatura específica (Johnson, 2001; Pride et al., 2004; Rubino et al., 2011; Masson y Pride, 2011; Quintal et al., 2011). Johnson (2001) estudió el comportamiento del módulo de volumen «undrained» efectivo de un medio poroso homogéneo que contiene «patches» binarios irregulares. Pride et al. (2004) analizaron los efectos de flujo inducido empleando soluciones analíticas para una serie de casos, incluyendo medios homogéneos saturados con 
«patches» binarios. Rubino et al. (2011) analizaron el efecto de distribuciones binarias de $\mathrm{CO}_{2}$ y agua en las trazas sísmicas, en el contexto de un proceso de inyección de $\mathrm{CO}_{2}$ en repositorios geológicos. Utilizando un algoritmo de invasión por percolación, Masson y Pride (2011) calcularon la atenuación sísmica en medios saturados de forma binaria por dos fluidos inmiscibles. Quintal et al. (2011) propusieron un método de diferencias finitas para estudiar los efectos del flujo inducido en la mesoescala y lo aplicaron a medios saturados por distribuciones binarias de fluidos. Más allá de estos estudios numéricos y teóricos, los modelos binarios de saturación suelen ser empleados para interpretar resultados experimentales. En este sentido, Tisato y Quintal (2013) emplearon un modelo de «patches» binarios de aire y agua para modelar los resultados de un experimento oscilatorio en una arenisca de la formación Berea. Recientemente, Chapman et al. (2016) utilizaron el modelo de White et al. (1975) para modelar los resultados de un experimento oscilatorio realizado en una muestra de arenisca de la formación Berea para diferentes presiones de confinamiento. Sin embargo, como ha sido mencionado previamente, las distribuciones de fluidos de escalas mesoscópicas observadas experimentalmente en muestras de roca, por medio de tomografía de rayos $\mathrm{X}$ $(\mathrm{CT})$, poseen variaciones espaciales suaves asociadas a las heterogeneidades del medio, con saturaciones de las fases que toman valores locales entre 0 y 1 (p. ej., Cadoret et al., 1998; Toms et al., 2007; Shi et al., 2011; Perrin y Benson, 2010; Li y Benson, 2015). Por lo tanto, es razonable cuestionar tanto la existencia de las distribuciones binarias de mesoescala de fluidos como la validez de las propiedades sísmicas derivadas en este tipo de escenarios.

Si bien los campos de saturación observados con CT en rocas porosas heterogéneas distan de ser binarios a escalas mesoscópicas, al analizar la distribución de los fluidos dentro del espacio poral es posible definir «burbujas» o «clusters» microscópicos. Estudios recientes han logrado mapear la distribución de fluidos inmiscibles dentro del espacio poral empleando microtomografías computadas de rayos $\mathrm{X}(\mu \mathrm{CT})$ (Rahman et al., 2016; Armstrong et al., 2014; Georgiadis et al., 2013; Iglauer et al., 2011). A modo de ejemplo, Rahman et al. (2016) tomaron imágenes de $\mu \mathrm{CT}$ de la distribución espacial de $\mathrm{CO}_{2}$ y agua en una muestra de arenisca Bentheimer $(10 \mathrm{~mm}$ de altura y $5 \mathrm{~mm}$ de diámetro), con una porosidad promedio de $22 \%$, luego de estadíos sucesivos de drenaje e imbibición. En este trabajo, las imágenes de $\mu \mathrm{CT}$ permiten medir el tamaño de los «clusters» de $\mathrm{CO}_{2}$ dentro del espacio poral. Podemos observar en la Figura 6.1a un corte 2-D de la roca empleada en este análisis, donde se observa un mapa segmentado de las fases fluidas y de la matriz sólida. $\mathrm{El} \mathrm{CO}_{2}$ está asociado a las regiones amarillas, el agua a las regiones azules y la matriz a las regiones grises. Se observa que los «clusters» de $\mathrm{CO}_{2}$ poseen diversas escalas, graficadas en 3-D en la Figura 6.1b. La Figura 6.1c muestra una imagen 3-D de un «cluster» de $\mathrm{CO}_{2}$ con un tamaño característico grande. Es interesante observar que, en general, el tamaño de los «clusters» está en el orden del tamaño de los granos y poros y, por lo tanto, no pueden ser considerados heterogeneidades mesoscópicas. Más allá de la escala de los mismos, puede observarse en la Figura 6.1a que el espacio poral que contiene a los «clusters» de $\mathrm{CO}_{2}$ se encuentra a su vez saturado con agua. Es decir, los «clusters» de $\mathrm{CO}_{2}$ ocupan el «centro» del espacio poral, y tienden a estar rodeados por una capa de agua que «moja» la paredes del poro. Este tipo de análisis de microescala brinda aún más razones para considerar incorrectos a los modelos binarios de saturación en la mesoescala. 
a)

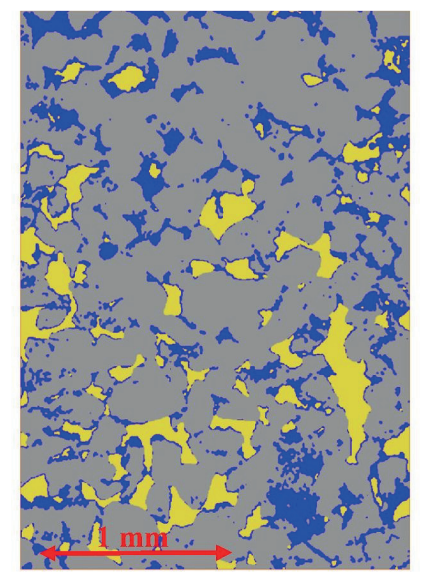

b)

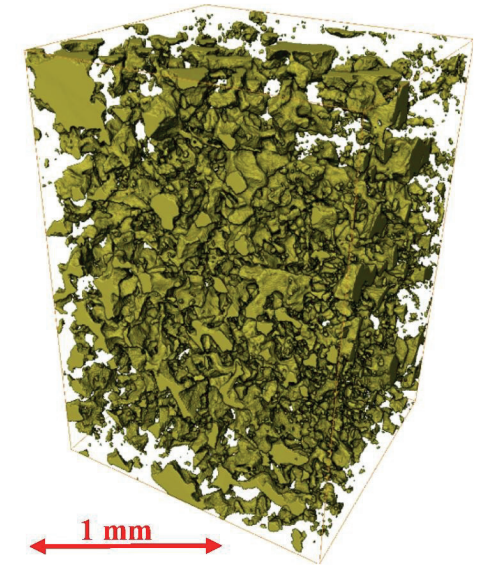

c)

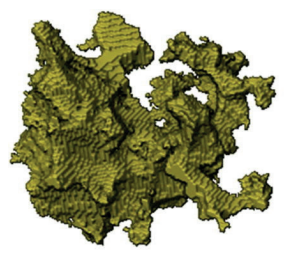

$500 \mu \mathrm{m}$

Figura 6.1. Imágenes de $\mu \mathrm{CT}$ de una muestra de roca arenisca de la formación Bentheimer saturada con $\mathrm{CO}_{2}$ y agua de formación a $10 \mathrm{MPa}$ y $318 \mathrm{~K}$ : (a) Corte 2-D de la muestra con las fases segmentadas. Los «clusters» de $\mathrm{CO}_{2}$ están asociados a las regiones color amarillo, el agua al color azul, y la matriz al gris; (b) «clusters» de $\mathrm{CO}_{2}$ en 3 -D, para un volumen de $3 \mathrm{~mm}^{3}$; (c) geometría de un «cluster» de $\mathrm{CO}_{2}$ de gran tamaño. Figuras tomadas de Rahman et al. (2016).

En los últimos años, se ha comenzado a explorar la respuesta sísmica de rocas saturadas por distribuciones continuas de dos fluidos inmiscibles, abandonando los modelos binarios de saturación. Rubino y Holliger (2012) exploraron la atenuación sísmica en rocas heterogéneas caracterizadas por campos de saturación continuos de agua y gas, asumiendo equilibrio capilar. Kuteynikova et al. (2014) modelaron la atenuación sísmica producida por flujo inducido por onda en la mesoescala en rocas homogéneas saturadas con distribuciones continuas de agua y aire. A su vez, en el Capítulo 5 de este trabajo de Tesis, hemos analizado el comportamiento de las curvas de atenuación y de dispersión de las ondas sísmicas durante un procesos de imbibición en un medio poroso heterogéneo. Sin embargo, ninguno de estos trabajos ha estudiado la capacidad de los modelos binarios de saturación para representar la respuesta sísmica de ambientes saturados con distribuciones realistas.

En este Capítulo, exploramos la capacidad de los modelos que consideran distribuciones binarias de mesoescala de fluidos para representar la atenuación y dispersión de las ondas sísmicas en medios que contienen distribuciones realistas de fluidos. Con este objetivo, en primer lugar analizamos la respuesta sísmica de un medio 1-D. Este 
consiste en una roca homogénea que contiene un frente de saturación resultante de un experimento de imbibición, logrado empleando el procedimiento numérico descripto en el Capítulo 4. Luego, determinamos la velocidad y la atenuación sísmica de este medio utilizando los procedimientos descriptos en los Capítulos 3 y 5 , es decir, empleando un test oscilatorio de relajación y un fluido efectivo. A su vez, analizamos el alcance del modelo de White para replicar las curvas de atenuación y dispersión asociadas. En un segundo análisis, estudiaremos un caso 2-D que consiste en una roca heterogénea cuyo campo de saturación resulta de un experimento de imbibición analizado en el Capítulo 5 de este trabajo de Tesis. Exploramos las consecuencias de tomar diferentes valores umbrales de binarización del campo de saturación en el contexto de la respuesta sísmica del medio, y analizamos si es posible obtener los valores de atenuación y dispersión del medio parcialmente saturado original empleando «patches» binarios de mesoescala.

\subsection{Respuesta sísmica de una roca homogénea 1-D parcialmente saturada}

\subsubsection{Muestra de roca y campo de saturación}

En esta Sección analizamos la capacidad del modelo de White para representar la respuesta sísmica de una roca que contiene un frente de saturación. Para ello simulamos un proceso de imbibición en una muestra 1-D de $1 \mathrm{~m}$ de largo, en la cual se inyecta agua a tasa constante. Se asume que la muestra está inicialmente saturada de forma homogénea con un $92 \%$ de $\mathrm{CO}_{2}$ y un $8 \%$ de agua. Las propiedades físicas de los fluidos están dadas en la Tabla 3.1, mientras que las propiedades de la muestra considerada se corresponden con las de una arenisca de la formación Utsira (ver Tabla 3.1). A su vez, utilizamos el modelo de Brooks y Corey, ecuaciones (4.9), (4.11), y (4.12), con $\lambda_{\mathrm{BC}}=2$ y $p_{d}=3450.18[\mathrm{~Pa}]$, y una saturación residual $S_{w, r}=0.05$. En cuanto a las condiciones de borde, imponemos una tasa de inyección en la parte superior de la muestra $(y=1 \mathrm{~m})$ igual a $q_{w}^{T}=5 \times 10^{-4} \mathrm{~m} / \mathrm{s}$, mientras que en la base imponemos una saturación $S_{w}^{B}=0.08$ y una presión $p_{w}^{B}=10[\mathrm{MPa}]$.

La Figura 6.2 muestra un instante del proceso de imbibición, a $t=600[\mathrm{~s}]$ desde el inicio del proceso de inyección. En este instante la roca posee una saturación global igual a $\left\langle S_{w}\right\rangle=75.6 \%$. Observamos que el campo de saturación toma la forma de un frente con características continuas, es decir, con valores locales de $S_{w}$ que varían entre 0 y 1. La muestra de roca considerada, saturada con el campo de la Figura 6.2, es sometida a un experimento oscilatorio de relajación. Es importante resaltar que consideramos una roca con matriz homogénea, por lo cual, el único contraste de compresibilidad presente en el medio se debe al frente de saturación. Empleamos el experimento oscilatorio de relajación descripto en el Sección 3.2 de esta Tesis para calcular la atenuación y la dispersión debidas a flujo inducido por onda a escalas mesoscópicas. Como cada celda del dominio posee una combinación de fases fluidas, debemos aplicar el procedimiento descripto en la Sección 5.2.3 de este trabajo de Tesis para obtener las propiedades efectivas del fluido poral.

Por otro lado, empleamos el modelo binario de capas planas paralelas de White para intentar representar la respuesta sísmica del medio. Es decir, buscamos una con- 


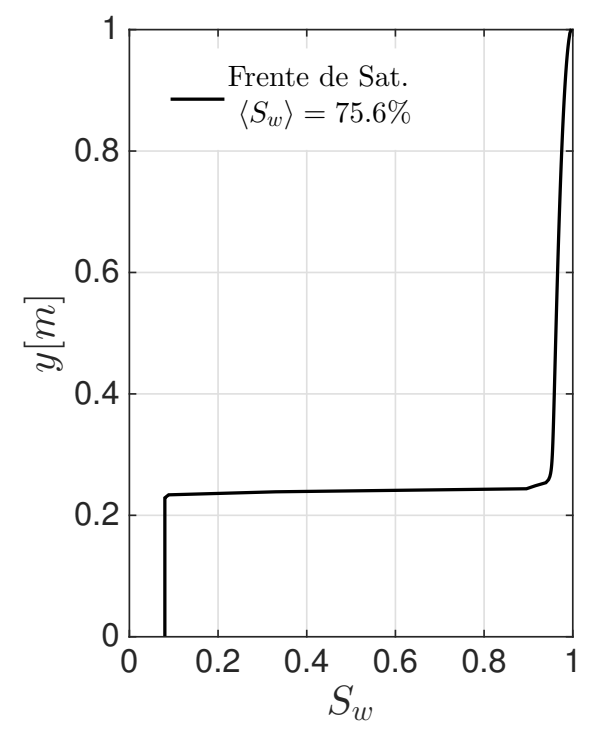

Figura 6.2. Frente de saturación a tiempo $t=600[\mathrm{~s}]$ en una arena Utsira.

figuración binaria que reproduzca la respuesta sísmica del modelo realista de la Figura 6.2. El modelo de White et al. (1975), descripto en el Sección 3.6.1, permite estimar el módulo de onda plana equivalente $M_{c}(\omega)$ (ecuación (3.61)). Luego, es posible obtener la atenuación y la dispersión para diferentes espesores de capas de $\mathrm{CO}_{2}$ y agua empleando las ecuaciones (3.1) y (5.15).

\subsubsection{Atenuación y dispersión de las ondas sísmicas}

La Figura 6.3 muestra el inverso del factor de calidad en función de la frecuencia para la roca saturada por el campo realista representado en la Figura 6.2, y para tres escenarios binarios de interés. Se grafica, en la Figura 6.3a, el campo de saturación realista, obtenido por medio de simulaciones de flujo, y la curva de atenuación en función de la frecuencia asociada al mismo (curva negra en la Figura 6.3e). El modelo graficado en la Figura $6.3 \mathrm{~b}$ es el modelo de White que presenta igual saturación global que la muestra realista, es decir $\left\langle S_{w}\right\rangle=75.6 \%$. Podemos observar que la curva de atenuación asociada a este modelo (curva azul en la Figura 6.3e) sobrestima considerablemente la atenuación del medio. Además, la frecuencia crítica del modelo binario es mayor que la del modelo realista.

Por otro lado, las Figuras 6.3c y 6.3d presentan los dos únicos modelos binarios de capas planas paralelas que poseen el mismo nivel de atenuación máximo $Q_{p, m a ́ x}^{-1}$ que el modelo realista. Estos modelos poseen saturaciones globales de $\left\langle S_{w}\right\rangle=31 \%$ y $\left\langle S_{w}\right\rangle=99.15 \%$, respectivamente. Es evidente que ambos casos poseen saturaciones completamente diferentes a las del medio realista. Además, ambos poseen una frecuencia crítica mayor a la del medio de la Figuras 6.3a. Hemos verificado que, considerando las propiedades físicas de la matriz y de los fluidos saturantes de este ejemplo, no existe un medio de White que posea la misma frecuencia crítica que el medio realista.

En la Figura 6.4 podemos observar la velocidad de onda $\mathrm{P}$ en función de la frecuencia para los diferentes casos analizados en la Figura 6.3. Se grafica, a su vez, el límite de 


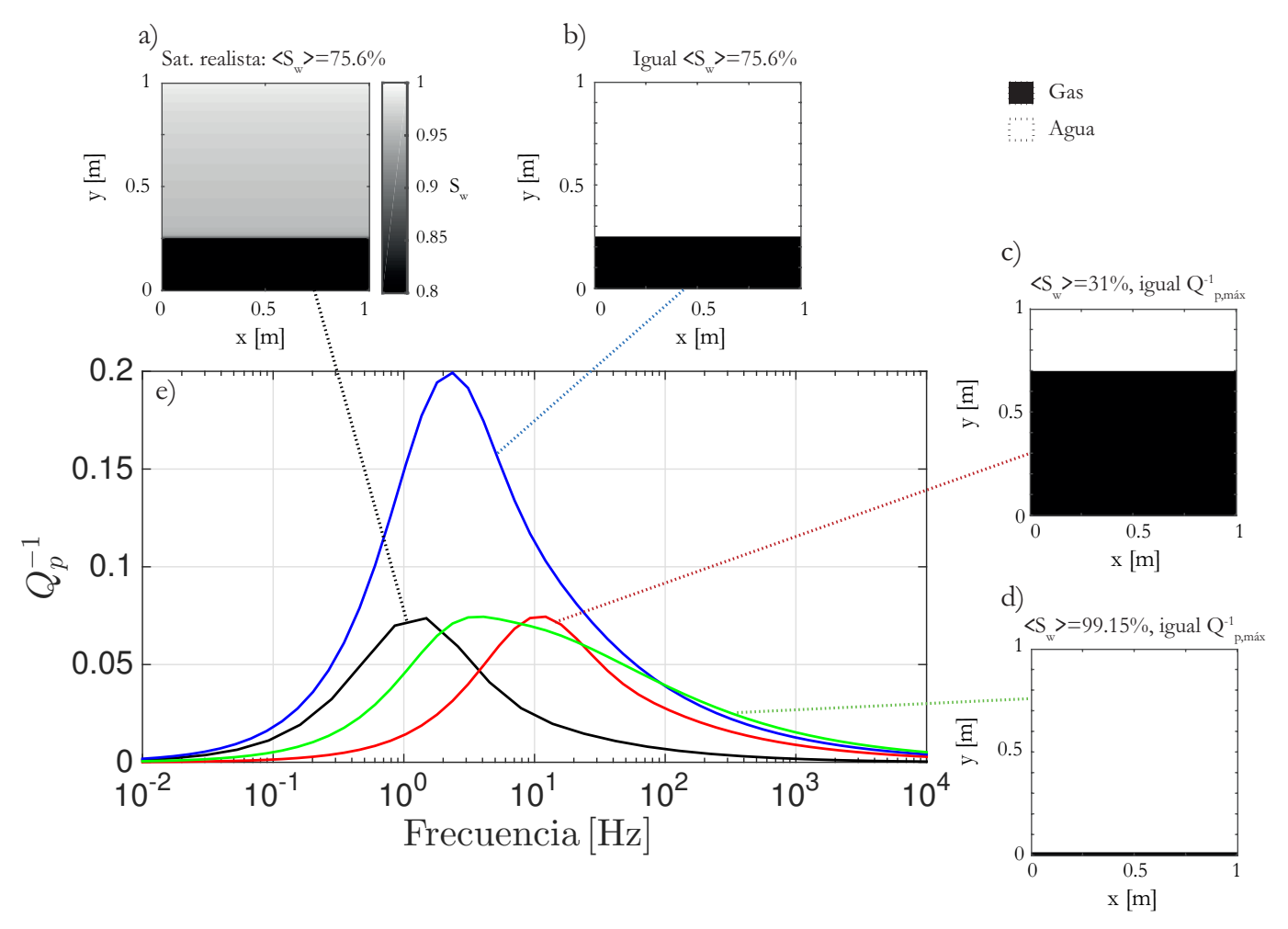

Figura 6.3. Inverso del factor de calidad de ondas compresionales en función de la frecuencia para el modelo de la Figura 6.2 y para diferentes modelos binarios de saturación.

bajas frecuencias de Gassmann-Wood (GW) empleando círculos negros. La dispersión asociada al medio realista está representada por una curva negra. Observamos que, al considerar el modelo binario de la Figuras 6.3b, el cual respeta la saturación global del medio original, la curva de dispersión asociada (curva azul) representa bien el comportamiento de la velocidad asociada al medio realista en el rango de frecuencias comprendido entre los $10^{-2} \mathrm{~Hz}$ y $1 \mathrm{~Hz}$, pero sobrestima considerablemente la dispersión por fuera de este rango.

Por otro lado, la dispersión asociada a los modelos binarios de las Figuras 6.3c y $6.3 \mathrm{~d}$, se encuentra graficada en la Figura 6.4 con curvas color rojo y verde, respectivamente. El modelo con igual atenuación máxima $Q_{p, \text { máx }}^{-1}$ y con $\left\langle S_{w}\right\rangle=31 \%$ (curva roja) presenta una inflexión en la curva de velocidad alrededor de los $100 \mathrm{~Hz}$, mientras que el medio realista posee una inflexión en $10 \mathrm{~Hz}$. Es interesante notar que, a pesar de poseer una saturación diferente a la del modelo realista, este modelo binario posee una velocidad similar en el rango de frecuencias comprendido entre los $10^{-2} \mathrm{~Hz}$ y $10^{-1} \mathrm{~Hz}$. Esto se debe a que la velocidad de GW, para estas propiedades de matriz y fluidos, varía muy poco $( \pm 10 \mathrm{~m} / \mathrm{s})$ al incrementar la saturación global $\left\langle S_{w}\right\rangle$ de $5 \%$ a $80 \%$. Para saturaciones globales mayores a $80 \%$ la velocidad aumenta considerablemente. Este es el caso del modelo binario con igual $Q_{p, \text { máx }}^{-1}$ y con $\left\langle S_{w}\right\rangle=99.15 \%$ (curva verde), cuya velocidad y dispersión son significativamente mayores que las del medio realista.

En base a lo observado en este análisis, es posible concluir que la utilización de modelos binarios deriva en estimaciones incorrectas de la atenuación y la velocidad de las ondas sísmicas. Observamos que ningún modelo binario puede representar el 


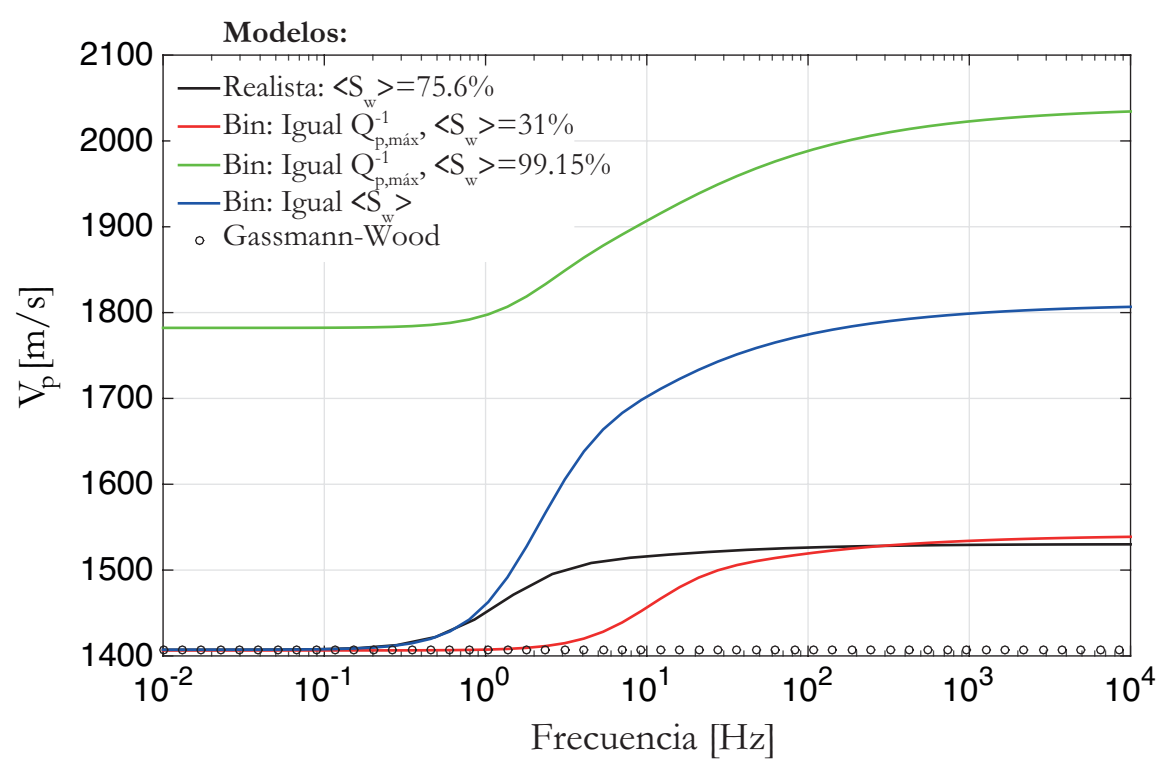

Figura 6.4. Velocidad de onda $\mathrm{P}$ en función de la frecuencia para los diferentes casos de la Figura 6.3.

comportamiento sísmico e imitar el valor de saturación global de la muestra, de forma simultánea. En particular, el modelo que mejor representa la distribución de fluidos del medio realista (Figura 6.3b), sobrestima la atenuación y la dispersión del medio que desea representar. A continuación analizaremos la contribución local a la atenuación con la intención de explorar las causas de las discrepancias observadas.

\subsubsection{Contribución local a la atenuación}

La contribución local a la atenuación permite observar las regiones de la roca que contribuyen significativamente a disipar la energía sísmica por flujo inducido. A continuación emplearemos los patrones espaciales de $q^{-1}(y)$ para analizar cuáles son las discrepancias entre el modelo binario de igual saturación (Figura 6.3b) y el modelo realista derivado de simulaciones de flujo (Figura 6.3a).

La Figura 6.5a presenta, de forma conjunta, el campo de saturación resultante del proceso de imbibición (curva negra) y el modelo de saturación binario previamente representado en la Figura 6.3b (curva discontinua azul). La contribución local a la atenuación global se calcula para la frecuencia asociada al máximo de atenuación $f_{c}$ correspondiente a cada modelo, es decir, $f_{c}^{\text {Real }}=1.4 \mathrm{~Hz}$ y $f_{c}^{\text {Bin }}=2.6 \mathrm{~Hz}$. Las contribuciones locales asociadas con el modelo realista $q_{\text {Real }}^{-1}$ y para el modelo binario $q_{\text {Bin }}^{-1}$ están graficadas en la Figura 6.5b empleando curvas color negro y azul, respectivamente. Podemos observar que el modelo binario presenta una contribución local con valores aproximadamente 4 veces mayores a los del modelo realista. Esto es esperable, ya que hemos observado que este sobrestima los niveles de atenuación y dispersión de la muestra. En particular, observamos en la Figura 6.5b que la principal diferencia se da en las cercanías de la interfase agua- $\mathrm{CO}_{2}(y=0.25 \mathrm{~m})$. Allí se observa una mayor contribución a la atenuación para el modelo binario, que se debe a un contraste de compresibilidad significativo de propiedades físicas entre ambas capas completamente saturadas. Esta característica da lugar a la generación de un gradiente de presión considerable en la 

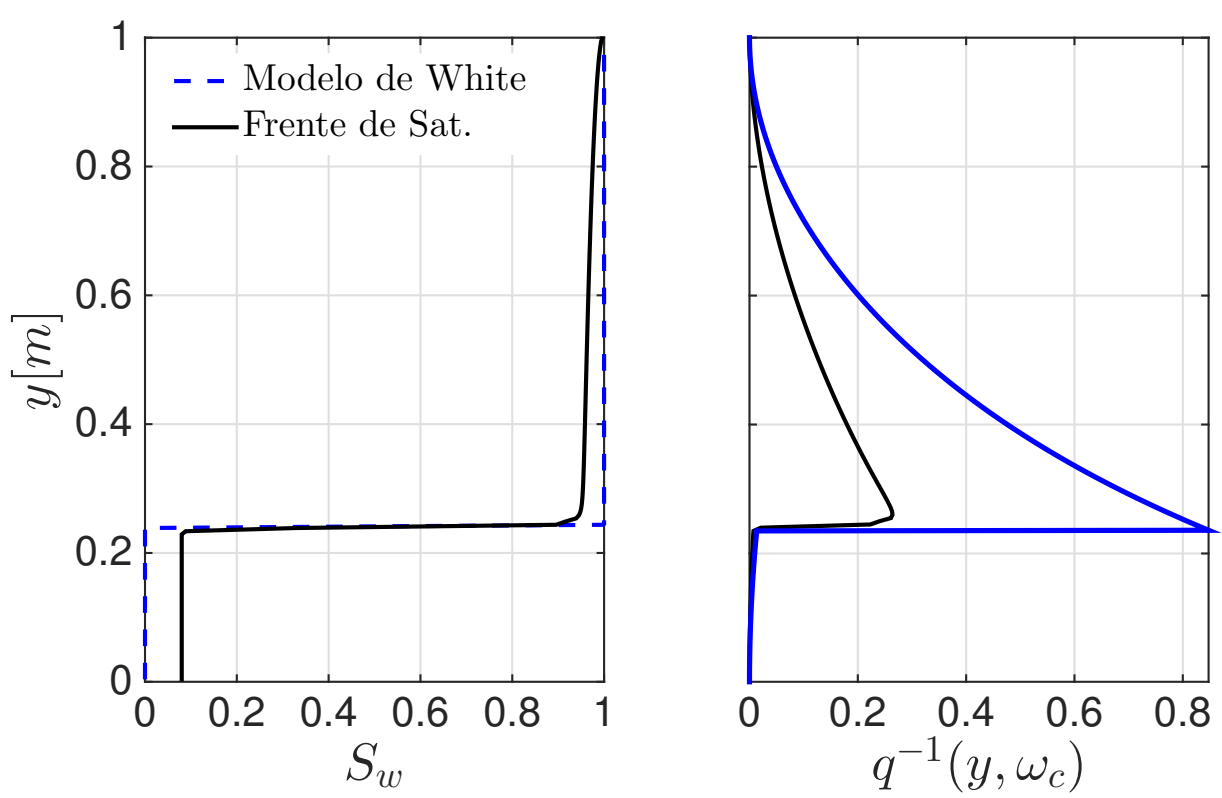

Figura 6.5. (a) Modelos de saturación binario (Figura 6.3b) y realista (Figura 6.2), representados por una curva azul discontinua y una curva negra sólida, respectivamente. (b) Contribución local a la atenuación para estos modelos.

interfaz entre ambas regiones. Para el modelo saturado de forma realista, las propiedades varían más gradualmente. Por lo cual, los gradientes de presión inducidos en el fluido son menores a los generados en el caso binario. Por estas razones, la atenuación y dispersión obtenidas al binarizar el campo de saturación son mayores.

\subsection{Respuesta sísmica de una roca heterogénea 2- D parcialmente saturada}

\subsubsection{Muestra de roca y campo de saturación}

A continuación comparamos la respuesta sísmica de una muestra de roca heterogénea 2-D que contiene un campo de saturación resultante de un proceso de imbibición, con la de un medio heterogéneo con un campo de saturación binario. Para generar una roca saturada de forma realista, tomamos los resultados del Capítulo 5 de este trabajo de Tesis. Es decir, consideramos la roca representada en la Figura 5.2b, saturada con una distribución de agua y metano resultante de un experimento de imbibición realizado con una tasa de inyección constante $q_{w_{1}}^{T}=1 \times 10^{-4}[\mathrm{~m} / \mathrm{s}]$. En particular, seleccionamos el estadío con saturación global $\left\langle S_{w}\right\rangle=94 \%$.

A diferencia del caso unidimensional de la Sección 6.2, donde empleamos un medio binario con geometría conocida (capas planas paralelas), la roca estudiada en esta Sección presenta una distribución bidimensional de fluidos altamente heterogénea. En consecuencia, es necesario definir un procedimiento que permita generar modelos binarios de saturación a partir del campo de saturación realista. Para ello, seguiremos el criterio empleado por Rubino y Holliger (2012) y Qi et al. (2014a), el cual consiste en 
derivar un campo binario a partir de una distribución continua de fluidos empleando el método de «thresholding». Este método, que ya ha sido empleado en la Sección 3 de este trabajo de Tesis para generar distribuciones binarias de fluidos, emplea un valor de saturación umbral $S_{w}^{\text {Thrs }}$ que sirve como criterio de corte para realizar la binarización. Es decir, se compara numéricamente el valor de saturación de agua de todas las celdas que conforman la roca de prueba numérica con el valor $S_{w}^{\text {Thrs }}$ elegido. Luego, toda celda cuyo valor de saturación sea mayor o igual a $S_{w}^{\text {Thrs }}$, se considera completamente saturada con agua. De lo contrario, toda celda cuya saturación es menor a $S_{w}^{\text {Thrs }}$ se considera completamente saturada con gas. Empleamos el método de «thresholding» para determinar una serie de campos binarios derivados de la muestra realista. Luego, calculamos la respuesta sísmica asociada a cada uno de ellos con la intención de explorar sus similitudes y diferencias respecto de la respuesta sísmica del medio realista original. Después de una búsqueda exhaustiva, considerando distintos valores umbrales $S_{w}^{\text {Thrs }}$, apartamos una serie de casos de interés, que serán analizados a continuación.

\subsubsection{Atenuación y dispersión de las ondas sísmicas}

La Figura 6.6 muestra las curvas de atenuación correspondientes al modelo de roca con saturación realista y una serie de modelos de saturación binaria. El esquema de la Figura 6.6a muestra el campo de saturación realista con $\left\langle S_{w}\right\rangle=94 \%$, resultante del experimento de imbibición previamente descripto. La curva de atenuación en función de la frecuencia de este medio, graficada en la Figura 6.6e, está representada utilizando una curva negra. El primer modelo binario de saturación analizado está dado por la Figura 6.6b, el cual posee igual saturación global que la roca original $\left(\left\langle S_{w}\right\rangle=94 \%\right)$. Para su determinación se emplea un valor umbral de $S_{w}^{\text {Thrs }}=79 \%$. La curva de atenuación en función de la frecuencia de este modelo (curva azul) evidencia una atenuación considerablemente mayor a la del modelo realista. Además, posee una frecuencia crítica menor.

Por otro lado, el modelo ilustrado en la Figura 6.6c responde a un valor umbral de $S_{w}^{\text {Thrs }}=88 \%$ y posee una saturación global de $\left\langle S_{w}\right\rangle=82.26 \%$. Lo interesante de este modelo es que posee una curva de atenuación (curva verde graficada en la Figura 6.6e) con una frecuencia crítica idéntica a la observada en la curva de atenuación de la roca saturada por el campo realista. Sin embargo, este modelo posee una saturación global menor, y presenta un nivel de atenuación mucho mayor a los valores asociados al modelo realista.

Por último, consideramos la muestra de la Figura 6.6d, que posee igual atenuación máxima que la roca saturada de forma realista (curva roja en la Figura 6.6e) y una saturación global de agua de $\left\langle S_{w}\right\rangle=99.88 \%$. Este modelo responde a un proceso de «thresholding» con un valor umbral de $S_{w}^{\text {Thrs }}=55 \%$. Podemos observar que la frecuencia crítica del modelo realista es mayor a la resultante de esta binarización. Además, al comparar las Figuras 6.6a y 6.6c observamos que la distribución de fluidos del modelo binario no es representativa de la distribución derivada de procesos de flujo. Asimismo, la saturación global del modelo binario asciende a $\left\langle S_{w}\right\rangle=99.88 \%$, por lo cual, el modelo binario sobrestima de forma considerable el nivel de saturación global de agua.

La Figura 6.7 presenta las curvas de velocidad de los modelos ilustrados en la 


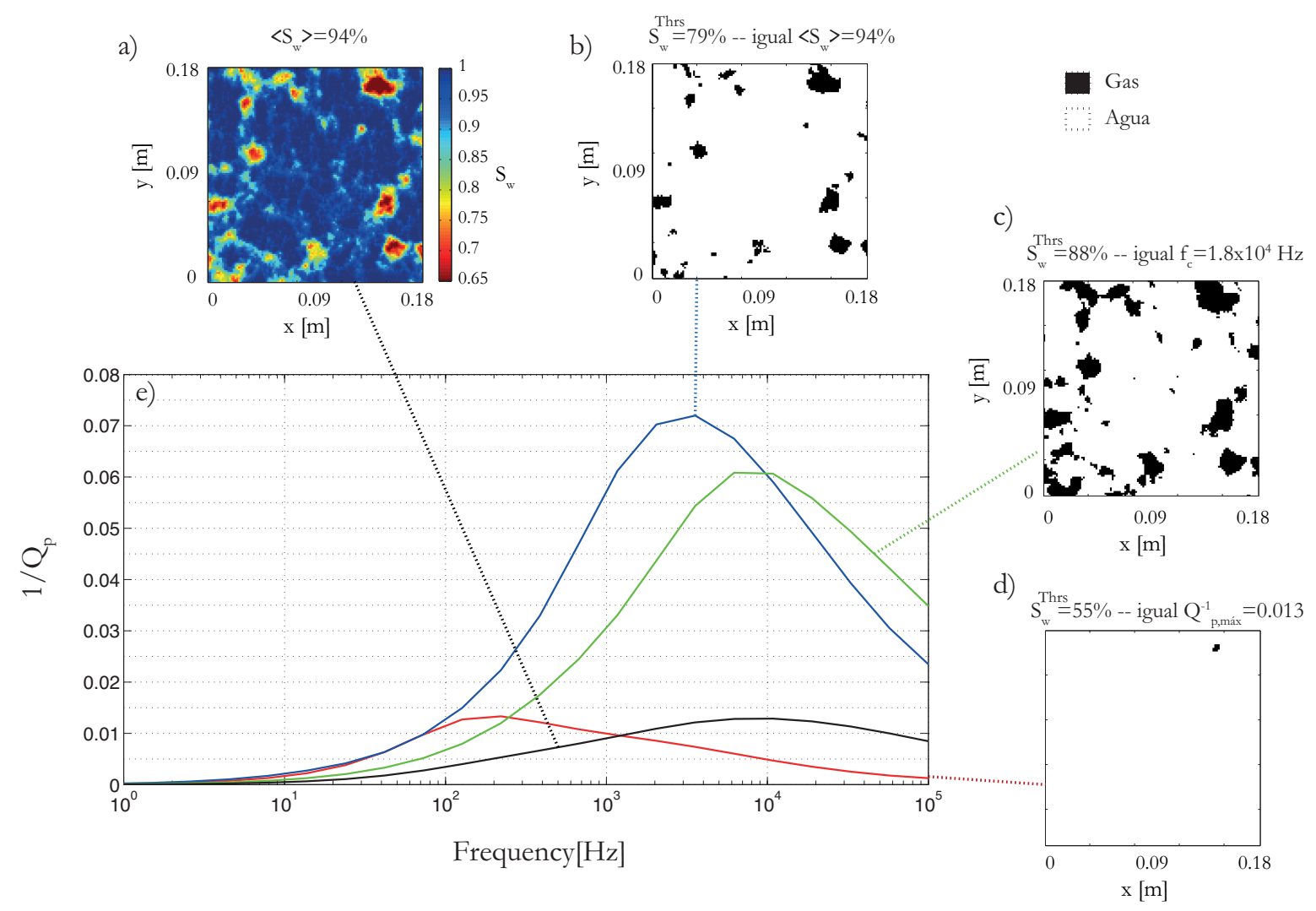

Figura 6.6. Inverso del factor de calidad de onda $\mathrm{P}$ en función de la frecuencia para diferentes modelos de saturación de la roca de prueba.

Figura 6.6. Podemos observar que el modelo realista presenta una dispersión menor a la de los modelos binarios de igual saturación (curva azul) y de igual frecuencia crítica (curva verde), que responden a las figuras 6.6b y 6.6c, respectivamente. Para frecuencias cercanas a $10^{5} \mathrm{~Hz}$, ambos modelos binarios sobrestiman la velocidad en más de $180 \mathrm{~m} / \mathrm{s}$. Es interesante notar que, a pesar de poseer saturaciones globales diferentes, ambos modelos binarios poseen velocidades similares a las del medio realista entre $1 \mathrm{~Hz}$ y $100 \mathrm{~Hz}$, las cuales se encuentran relativamente bien modeladas por la aproximación de GW (círculos negros) considerando una saturación global de $\left\langle S_{w}\right\rangle=94 \%$. Esto se debe a que la velocidad de GW no varía significativamente al pasar de una saturación global de agua de $82.26 \%$, asociada modelo representado en la Figura 6.6c, a una saturación global de $94 \%$, asociada a los modelos de las Figuras 6.6 a y 6.6b.

Por otro lado, en la Figura 6.7 se grafica la velocidad de fase asociada al modelo binario que posee igual atenuación máxima que el modelo realista. Observamos que el comportamiento de la velocidad con la frecuencia (curva roja) es absolutamente diferente a la del medio realista. Además, como al binarizar con este criterio obtenemos un medio saturado con $\left\langle S_{w}\right\rangle=99.88 \%$, el valor de las velocidades en el límite de bajas frecuencias es significativamente diferente.

Podemos concluir entonces que, al igual que en el caso 1-D previamente analizado, las curvas de atenuación y dispersión por flujo inducido asociadas a medios que contienen campos de saturación binarios, obtenidos por «thresholding», no pueden satisfacer de forma simultánea los criterios de igual saturación global, igual nivel de 


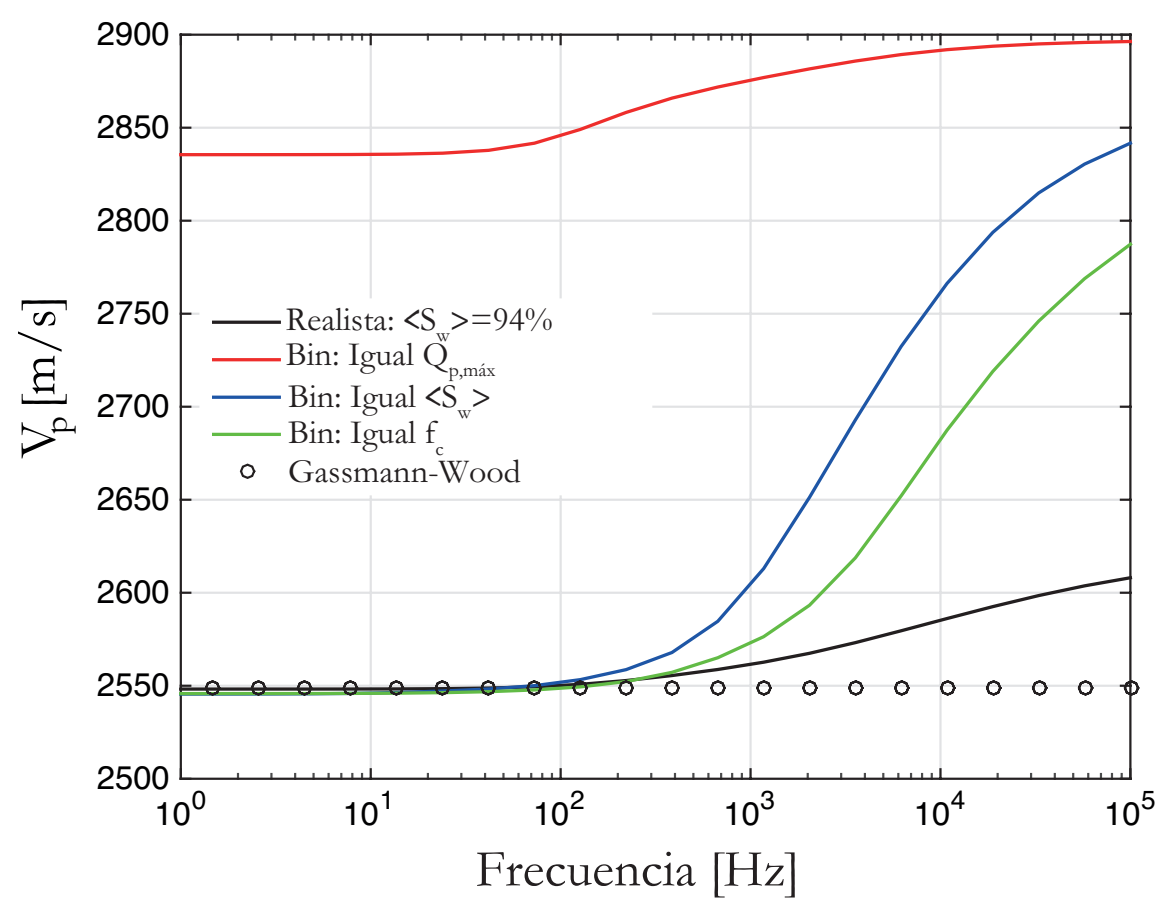

Figura 6.7. Velocidad de onda $\mathrm{P}$ en función de la frecuencia para los diferentes casos de la Figura 6.6.

atenuación y dispersión, e igual frecuencia crítica que la muestra saturada por medio de un proceso de flujo. En particular, el modelo binario que posee igual saturación que la muestra realista sobrestima considerablemente los niveles de atenuación y dispersión sísmica. Estos ejemplos nos llevan a cuestionar la validez de las curvas de atenuación y dispersión obtenidas empleando modelos binarios de saturación. A continuación, con la intención de explorar las diferencias en los patrones de disipación asociados a los diferentes modelos, estudiaremos la contribución local a la atenuación.

\subsubsection{Contribución local a la atenuación}

En esta Sección analizaremos cuáles son las regiones de la roca que contribuyen más significativamente a disipar la energía sísmica por flujo inducido para los modelos 2-D previamente considerados. Con esta motivación, exploraremos las características de la contribución local a la atenuación $q_{i j}^{-1}$.

Para realizar una comparación de los patrones de $q_{i j}^{-1}$ seleccionamos el modelo realista representado por la Figura 6.6a y los dos modelos binarios representados en las Figuras 6.6b (modelo de igual saturación) y 6.6c (modelo de igual frecuencia crítica). No analizaremos el modelo de la Figura $6.6 \mathrm{~d}$ ya que, a pesar de presentar igual valor para $Q_{p, m a ́ x}^{-1}$, la distribución de fluidos asociada es poco representativa del medio realista.

En las Figuras 6.8a, 6.8b, y 6.8c graficamos los campos de saturación para estos modelos de roca saturada. A su vez, debajo de estos, se grafica la contribución local a la atenuación en cada caso, calculada en la frecuencia crítica correspondiente (Figuras 6.8d, 6.8e, y 6.8f). Realizando una comparación conjunta de las distribuciones de fluidos en las Figuras 6.8a y 6.8b, y de los patrones de $q_{i j}^{-1}$ correspondientes (Figuras 6.8d y 


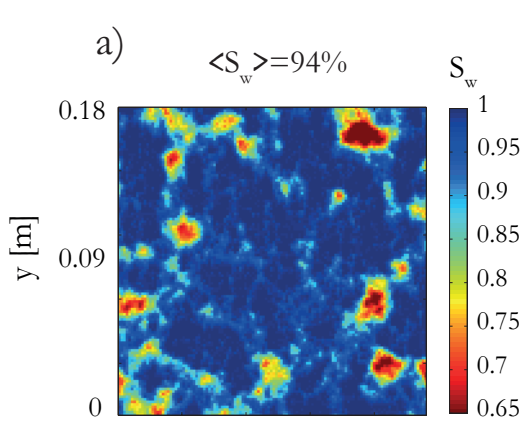

d)

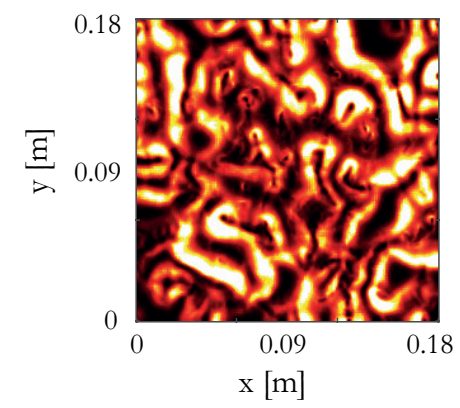

b) Igual $\left\langle\mathrm{S}_{\mathrm{w}}>=94 \%\right.$

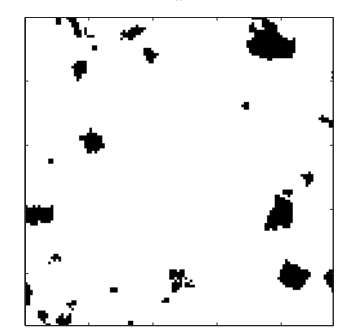

e)

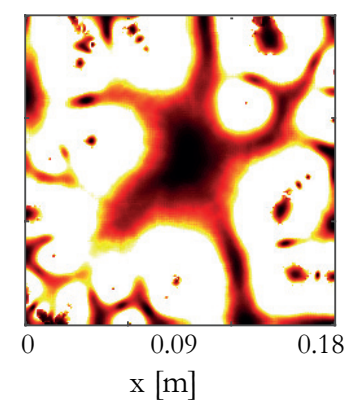

c) Igual $\mathrm{f}_{\mathrm{c}}=1.8 \times 10^{4} \mathrm{~Hz}$

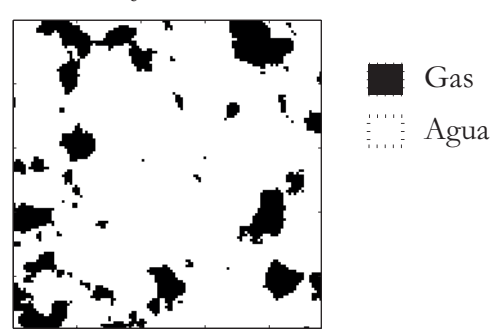

f)

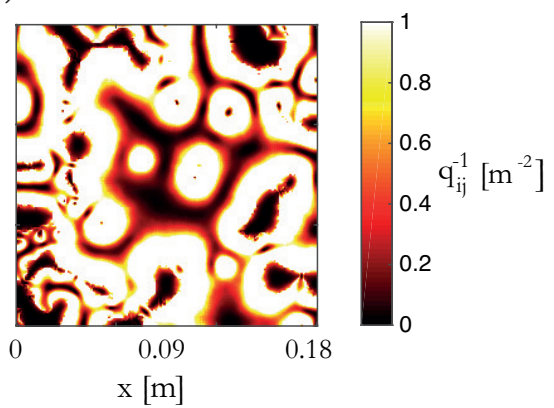

Figura 6.8. Campos de saturación y contribución local a la atenuación $q_{i j}^{-1}\left(\omega_{c}\right)\left[\mathrm{m}^{-2}\right]$. Los campos de saturación graficados en los paneles (a), (b), y (c) están asociados a los modelos de las Figuras 6.6a, 6.6b, y 6.6c. Los paneles (d), (e) y (f) muestran la contribución local a la atenuación asociada a los modelos correspondientes que se encuentran en la fila superior.

6.8e), observamos que localmente los valores de $q_{i j}^{-1}$ son mayores para el modelo binario. esto se debe a que el contraste de compresibilidad es mayor y, entonces, el flujo inducido es más importante. Además, la superficie total de las regiones contribuye de forma significativa a la atenuación es también mayor en el modelo binario, lo que explica que la atenuación global sea mayor. Lo mismo sucede al comparar la contribución local del modelo binario de igual frecuencia crítica (Figura 6.8f) con el del modelo realista. En particular, observamos que cuanto más compleja es la distribución de gas dentro del medio, mayor es el proceso de interacción entre patches y, entonces, la extensión espacial del proceso difusivo se ve reducida. Más allá de esto, observamos que ninguno de los modelos binarios es capaz de representar las características de la distribución espacial de $q_{i j}^{-1}$ del modelo realista.

Como se ha observado en este análisis, los modelos binarios no pueden representar de manera simultánea los niveles de atenuación y dispersión, la saturación global de la muestra, y la frecuencia crítica asociada a un modelo realista. Consecuentemente, en el eventual caso de utilizar modelos binarios en un esquema de inversión a partir de datos sísmicos, se obtendrán estimaciones erróneas del contenido de fluido, de las características de su distribución espacial, y de las propiedades hidráulicas de la matriz. Estos resultados resaltan la necesidad de avanzar hacia modelos realistas de roca saturada al realizar modelado de propagación de ondas sísmicas y al interpretar los datos correspondientes. 


\subsection{Limitaciones técnicas y prácticas asociadas al cálculo de los efectos de flujo mesoscópico en medios parcialmente saturados}

El flujo inducido por onda es actualmente considerado como un mecanismo de atenuación dominante en medios porosos parcialmente saturados, siempre y cuando el campo de saturación presente heterogeneidades de tamaño mesoscópico y las fases fluidas posean compresibilidades contrastantes. Las mediciones de laboratorio realizadas en rocas con heterogeneidades mesoscópicas, empleando frecuencias sísmicas y sónicas, indican la existencia de niveles de atenuación y dispersión sísmica medibles que suelen atribuirse a flujo mesoscópico (Yin et al., 1992; Cadoret et al., 1998; Tisato y Quintal, 2013; Chapman et al., 2016). Sin embargo, el rol que juegan los efectos del flujo mesoscópico en experimentos de campo es aún incierto. De hecho, desde la introducción del mecanismo de atenuación de flujo inducido por onda en medios porosos en los trabajos de White (1975) y White et al. (1975), los modelos desarrollados no han sido aún acoplados a esquemas de inversión eficientes.

La falta de aplicación de los modelos de flujo mesoscópico en escenarios de campo posee varias razones. En primer lugar, las mediciones de atenuación son difíciles de realizar, y la incerteza en las mismas aumenta rápidamente a medida que la atenuación disminuye (Sams et al., 1997). Además, las mediciones de campo se suelen realizar en un entorno de banda limitada, lo cual condiciona el análisis de la variación de la atenuación y velocidad con la frecuencia. En este sentido, aunque se desarrolle un modelo adecuado para la atenuación y la velocidad observadas en el rango de frecuencias considerado, la implementación del mismo requiere de mediciones en un amplio rango de frecuencias para verificar que sus estimaciones sean correctas. Sams et al. (1997) reportaron los resultados de una serie de experimentos en un amplio rango de frecuencias, que incluyen: Perfilaje sísmico vertical (VSP) $(30$ a $280 \mathrm{~Hz}$ ), datos sísmicos de «crosshole» (200 a $2300 \mathrm{~Hz}$ ), sónicos ( 8 a $24 \mathrm{kHz}$ ), y mediciones de laboratorio (0.3 a $0.9 \mathrm{MHz}$ ). Empleando estos datos, Pride (2005) propuso un modelo de doble porosidad que explica la atenuación observada en todos los rangos de frecuencia considerando disipación por flujo mesoscópico. El modelo propuesto consiste en una arenisca porosa consolidada fracturada con «cracks» mesoscópicas elípticas de radio igual a $5 \mathrm{~cm}$. Sin embargo, no existe evidencia de que este modelo haya sido verificado en el sitio de investigación, y como será argumentado a continuación, es poco probable que la atenuación asociada a un rango de frecuencias tan amplio esté únicamente asociada a disipación por flujo mesoscópico.

Por otro lado, como se ha resaltado previamente, la disipación sísmica asociada al mecanismo de flujo inducido por onda está relacionada con una gran cantidad de parámetros del medio, tales como la porosidad, la permeabilidad, la longitud característica de las heterogeneidades, las propiedades físicas de los fluidos saturantes. Algunos de estos parámetros, tales como, por ejemplo, la permeabilidad, son parámetros muy difíciles de determinar y que pueden variar varios órdenes de magnitud en cortas distancias. Por lo cual, si se desea llevar a cabo una inversión de datos sísmicos en el marco de flujo mesoscópico, es preciso contar con una gran cantidad de información de las formaciones analizadas. En líneas generales, debe contarse con estimaciones de 
la porosidad efectiva (p. ej., empleando perfilajes de densidad), de la saturación (p. ej., empleando perfilajes eléctricos), y de la composición litológica (analizando coronas, muestras de roca, o perfiles de pozo). Además, el muestreo de estas propiedades físicas debe ser llevado a cabo a escalas significativamente menores que las longitudes de onda utilizadas, que en el caso de un sónico suelen encontrarse en las decenas de cm. Esta característica suele ser altamente limitante, y por ello comúnmente se recurre modelos de física de rocas para obtener las propiedades en las escalas deseadas (p. ej., Milani et al., 2015). Un esfuerzo interpretativo en esta dirección fue dado por Caspari et al. (2011). Los autores de este trabajo emplearon datos sónicos de un experimento de almacenamiento de $\mathrm{CO}_{2}$ en Nagaoka para estimar una relación velocidad-saturación de $\mathrm{CO}_{2}$ para diferentes profundidades del reservorio. Con esta motivación, se propuso un modelo de flujo mesoscópico de «patches» binarios de $\mathrm{CO}_{2}$ que logra explicar el comportamiento observado. Para ello se utilizó el modelo de «patches» aleatorios CRM, considerando tamaños característicos para los «patches» binarios que varían entre 1 y $5 \mathrm{~mm}$. Sin embargo, como hemos observado en este Capítulo, los modelos de «patches» binarios tienden a ser poco representativos de las características del medio estudiado.

Por último, existe una serie de mecanismos adicionales que afectan las amplitudes sísmicas en los medios porosos heterogéneos saturados, tales como el «squirt flow», el mecanismo intrínseco de Biot, el «scattering», la anaelasticidad de la matriz, y la divergencia geométrica. Es importante resaltar que existen trabajos donde se realizaron esfuerzos para modelar los efectos combinados de varios de estos procesos disipativos. Milani et al. (2015) propusieron explorar la atenuación y dispersión sísmica empleando información de un perfilaje sónico de multifrecuencia (1 a $30 \mathrm{kHz}$ ) realizado en sedimentos aluviales saturados por agua (Baron y Holliger, 2011). El modelo desarrollado considera los efectos del mecanismo intrínseco de Biot, de «squirt flow», y del flujo mesoscópico asociado a cambios en la porosidad de los sedimentos monosaturados. Para ello, en primer lugar, las mediciones deben corregirse por divergencia esférica. Luego, una conjunción de datos de perfilaje complementarios y modelos de física de rocas permiten estimar los parámetros poroelásticos necesarios para calcular y ajustar las mediciones de atenuación y dispersión sísmica observadas. Los autores resaltan que el flujo mesoscópico no posee un rol importante en este contexto, lo cual es esperable ya que no existen contrastes de compresibilidad considerables asociados a fracturas y/o a saturación parcial. Recientemente, Sayar y Torres-Verdín (2017) han propuesto un modelo de medio efectivo que considera una amplia gama de mecanismos de disipación. Entre ellos se destacan el flujo mesoscópico asociado a la presencia de fracturas, al mecanismo intrínseco de Biot, y a «scattering» asociado a la presencia de inclusiones esféricas y esferoidales orientadas aleatoriamente. Este modelo está basado en los modelos de flujo mesoscópico de Chapman et al. $(2002,2006)$ y considera que la disipación mesoscópica está asociada a relajación de presión poral entre fracturas elipsoidales y su entorno. El trabajo de Sayar y Torres-Verdín (2017) provee de una metodología de fácil aplicación, lo cual es importante en un potencial esquema de inversión. En este sentido, los costos computacionales asociados al modelado numérico de los efectos disipativos poroelásticos, tales como, la realización de experimentos oscilatorios numéricos de relajación, suelen ser demasiado altos. Debido a esta razón, a pesar de sus limitaciones, las soluciones analíticas, tales como el modelo de Johnson (2001) o el modelo de White et al. (1975) analizado en el Capítulo 3 de este trabajo de Tesis, poseen un 
mayor potencial para ser incluidas en esquemas de inversión.

Por estas razones, de todas las posibles técnicas sísmicas, los experimento de laboratorio son los que tienen un mayor potencial para ser interpretados utilizando los modelos presentados en los Capítulos 5 y 6 de este trabajo de Tesis. En particular, los mismos deben llevarse a cabo utilizando técnicas de oscilación subresonantes (p. ej., Tisato y Madonna, 2012; Chapman et al., 2016). Además, se debe poseer la capacidad de controlar la tasa de inyección de fluidos, lo cual es una práctica común en varios estudios experimentales (p. ej., Lopes y Lebedev, 2012; Lopes et al., 2014). La determinación de la importancia de los efectos del flujo mesoscópico en los experimentos de laboratorio permitiría también, analizando las discrepancias entre modelo y dato, determinar la existencia de otros mecanismos de atenuación. A continuación presentaremos un posible flujo de trabajo para analizar los niveles de atenuación y dispersión por flujo inducido por onda en la mesoescala en medios parcialmente saturados durante procesos de inyección de fluidos. Este esquema ha sido implementado en los Capítulos 5 y 6 de esta Tesis para estudiar las propiedades sísmicas en medios saturados por patrones realistas y se presenta como una alternativa superadora a los modelos basados en «patches» binarios de fluidos.

\subsection{Recomendaciones de aplicación práctica}

La Figura 6.9 resume nuestras recomendaciones prácticas para la implementación de los modelos de flujo mesoscópico durante procesos de inyección de fluidos. Este flujo de trabajo permite partir de las propiedades hidráulicas y mecánicas de una muestra de roca de laboratorio, de dimensiones $L_{x} \times L_{y}$, y estimar la atenuación $Q_{p}^{-1}(\omega)$ y dispersión $V_{p}(\omega)$ considerando patrones realistas de saturación.

Previo a la implementación del método, se requiere de una serie procedimientos que permitan obtener las propiedades hidráulicas y mecánicas de la roca de prueba. En primer lugar, la distribución de la porosidad media de la muestra de roca $\phi(\mathbf{x})$ y la saturación inicial $S_{w}^{\text {ini }}(\mathbf{x})$ pueden ser calculadas empleando tomografías de rayos X (p. ej., Shi et al., 2011; Krevor et al., 2011; Alemu et al., 2013). Las saturaciones residuales $S_{r, w}, S_{r, n}$ de las fases pueden obtenerse analizando las saturaciones mínimas de ambas fases empleando tomografías de rayos $\mathrm{X}$ o bien empleando modelos de física de rocas presentes en la literatura (p. ej., Mavko et al., 2009). Las propiedades mecánicas efectivas de los granos sólidos $K_{s}, \mu_{s}$ y $\rho_{s}$ pueden obtenerse analizando la composición mineralógica de la muestra de roca determinada mediante un análisis de difracción de rayos X (Alemu et al., 2013). La tensión interfacial $\gamma$ y las propiedades físicas de los fluidos saturantes $K_{n}, K_{w}, \rho_{n}, \rho_{w}, \eta_{n}, \mathrm{y} \eta_{w}$, pueden obtenerse de tablas presentes en la literatura específica considerando las temperaturas y presiones utilizadas en los experimentos correspondientes (p. ej., Nakagawa et al., 2013; Batzle y Wang, 1992).

El flujo de trabajo puede resumirse en los siguientes pasos:

- En primer lugar, se determinan las propiedades mecánicas de la roca seca $K_{m}(\mathbf{x})$ y $\mu_{m}(\mathbf{x})$, la presión de entrada $p_{d}(\mathbf{x})$, y la permeabilidad $\kappa(\mathbf{x})$. Hemos comprobado que la variación espacial de las propiedades mecánicas de la roca seca posee un efecto despreciable en las mediciones de atenuación y dispersión en medios parcialmente saturados. Por ello recomendamos emplear un único valor para toda la 
muestra numérica, es decir, $K_{m}(\mathbf{x}) \equiv\left\langle K_{m}\right\rangle$ y $\mu_{m}(\mathbf{x}) \equiv\left\langle\mu_{m}\right\rangle$. Estos valores a escala muestra pueden obtenerse a partir de experimentos de laboratorio realizados sobre la roca seca. La presión de entrada y la permeabilidad pueden obtenerse en cada celda empleando modelos de física de rocas apropiados (p. ej., Pride, 2005; Leverett, 1941; Kozeny, 1927; Carman, 1937).

- Luego, se utiliza un código que simule el flujo bifásico en dicho medio, considerando las condiciones de inyección seleccionadas. El mismo está complementado por modelos constitutivos determinados (p. ej., Brooks y Corey, 1964; van Genuchten, 1980). De esta forma se obtiene el campo de saturación $S_{w}(\mathbf{x})$ luego de un tiempo determinado de inyección $t$.

- El tercer paso está dado por la determinación de las propiedades efectivas del fluido saturante $K_{f}(\mathbf{x}), \eta_{f}(\mathbf{x})$ y $\rho_{f}(\mathbf{x})$ utilizando relaciones adecuadas (p. ej., Teja y Rice, 1981; Wood, 1955).

- Finalmente, se determinan los parámetros poroelásticos $\alpha(\mathbf{x}), M(\mathbf{x})$, y $\lambda_{c}(\mathbf{x})$ y la densidad promedio del agregado $\rho_{b}(\mathbf{x})$. A través de los mismos es posible obtener estimaciones de la velocidad $V_{p}(\omega)$ y de la atenuación $Q_{p}^{-1}(\omega)$ sísmica por flujo mesoscópico empleando el test oscilatorio de relajación descripto en el Capítulo 3. La frecuencia angular $\omega$ puede variarse en el rango de frecuencias del experimento considerado.

Es importante resaltar que el acuerdo entre las mediciones realizadas y los resultados del esquema aquí propuesto dependerán de los modelos de física de rocas empleados. Por lo cual, es esperable que sea necesario un ajuste de los parámetros de los mismos si se desea representar adecuadamente los datos. En particular, la principal limitación en la aplicación de este modelo se encuentra en la estimación del campo de permeabilidad $\kappa(\mathbf{x})$, ya que este no puede medirse con el instrumental actual y es un parámetro clave en los procesos de flujo. Existen diferentes relaciones empíricas para estimar la permeabilidad a partir del campo de porosidad $\phi(\mathbf{x})$ que pueden ser utilizadas (p. ej., Kozeny, 1927; Carman, 1937; Krause et al., 2009, 2013). En este sentido, se recomienda repetir el proceso considerando distintas relaciones empíricas, con la intención de analizar el impacto de la incertidumbre asociada a la estimación de este parámetro en los valores de $Q_{p}^{-1}(\omega)$ y $V_{p}(\omega)$.

El esquema de trabajo propuesto en esta sección tiene como objetivo proveer de una estimación de los niveles de atenuación y dispersión por flujo mesoscópico en rocas parcialmente saturadas. De hecho, diversos trabajos han propuesto distribuciones binarias de fluidos con este propósito. Por ejemplo, Tisato y Quintal (2013) midieron la atenuación sísmica en una roca Berea luego de un proceso de imbibición, considerando agua y aire, empleando técnicas de oscilación subresonantes. Estos autores propusieron como mecanismo de atenuación predominante al flujo inducido a escala mesoscópica debido a la existencia de heterogeneidades en el campo de saturación, y emplearon un modelo de «patches» binarios para modelar sus datos. Como hemos observado en este Capítulo, los modelos binarios de saturación tienden a estar asociados a estimaciones erróneas de las características del medio. En este contexto, el presente modelo podría permitir comprender la importancia del flujo mesoscópico empleando distribuciones 
más realistas. En particular, las posibles discrepancias con los datos podrían ser indicadores de que otros mecanismos de atenuación, como el «squirt flow», deben ser considerados.

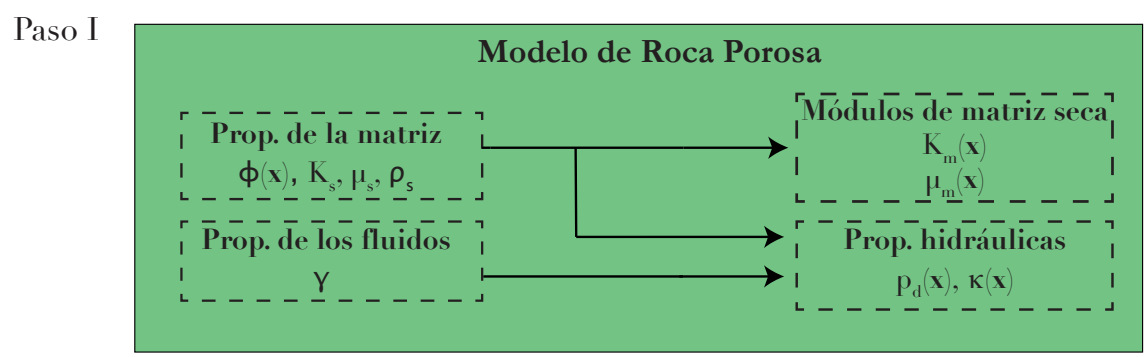

Paso II

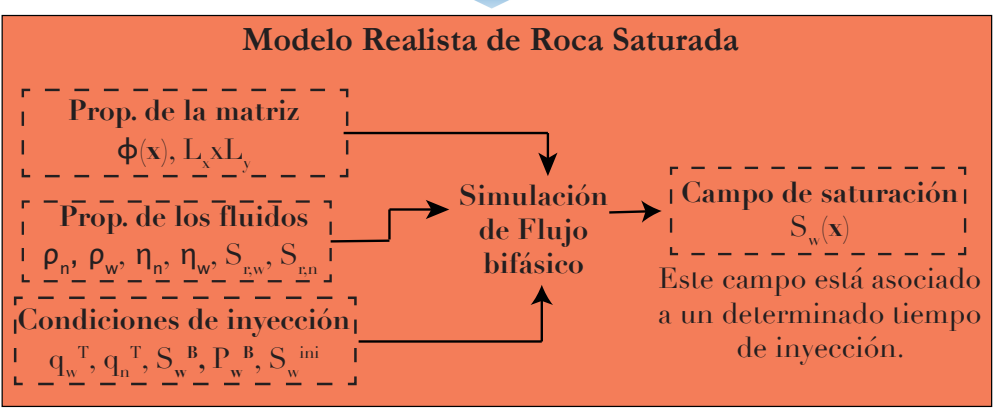

Paso III

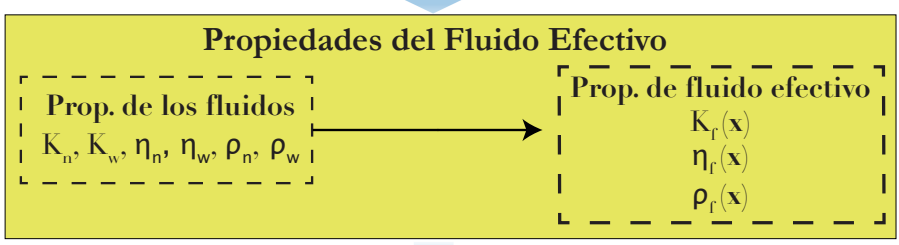

Paso IV

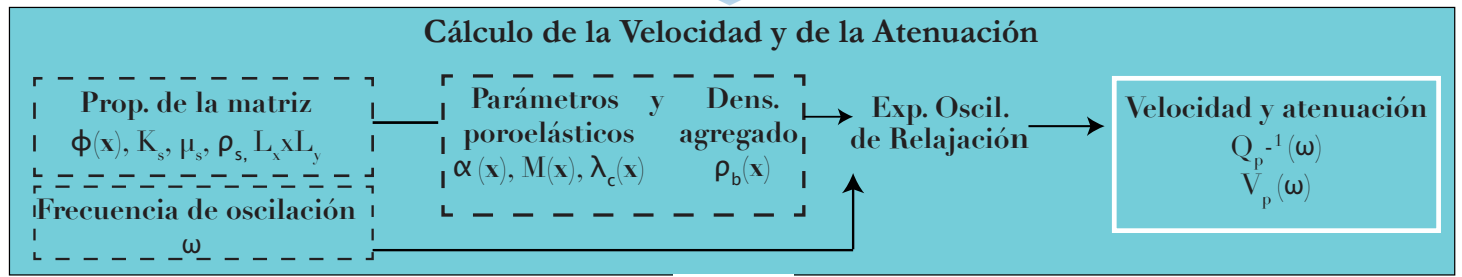

Comparación entre $Q_{\mathrm{p}}{ }^{-1}(\omega) \mathrm{y}$

$V_{p}(\omega)$ y mediciones de velocidad y atenuación de laboratorio

Figura 6.9. Flujo de trabajo para calcular la atenuación y dispersión por flujo inducido en la mesoescala empleando campos de saturación realistas. 


\subsection{Conclusiones}

En este Capítulo hemos analizado la capacidad de los modelos binarios para representar la atenuación y dispersión sísmica debido a flujo inducido por onda en la mesoescala asociada a modelos con distribuciones realistas de fluidos inmiscibles. En primer lugar, hemos empleado el modelo de White para intentar reproducir la respuesta sísmica de un frente de saturación resultante de un experimento de imbibición realizado en una roca homogénea. Luego, hemos generado distribuciones binarias de saturación a partir de un modelo 2-D de saturación realista, derivado de un proceso de imbibición en una roca heterogénea. Los resultados evidencian que las estimaciones obtenidas por medio de modelos binarios no han podido representar, de manera simultánea, los niveles de atenuación y dispersión, el nivel de saturación global, y la frecuencia crítica de la muestra saturada de forma realista.

Un análisis detallado de las características de la contribución local a la atenuación nos permite observar que los modelos binarios más representativos, es decir, aquellos cuya distribución de fluidos se asemeja a la distribución original, tienden a sobreestimar los niveles de disipación local alrededor de los «patches» en comparación con los valores asociados a modelos realistas. Esto hace que la atenuación y la disipación sísmica resultante tienda a ser considerablemente mayor en comparación con los efectos asociados a los campos de saturación realista correspondientes. Por estas razones, si se emplean modelos binarios para interpretar la respuesta sísmica de un medio poroso parcialmente saturado, se puede incurrir en estimaciones erróneas del contenido de fluidos, la geometría de los «patches», y de las propiedades hidráulicas de la matriz. Estos resultados permiten afirmar que, si se desea modelar correctamente la atenuación y dispersión sísmica por flujo inducido en medios parcialmente saturados, es preciso considerar patrones de fluidos realistas. Asimismo, en base a este análisis, gran parte de las conclusiones obtenidas en los trabajos de la literatura empleando distribuciones binarias de fluidos deberían reverse o cuestionarse.

Por último, se propone un posible flujo de trabajo para analizar los niveles de atenuación y dispersión por flujo mesoscópico en medios parcialmente saturados durante procesos de inyección de fluidos. El mismo se presenta como una alternativa superadora a los modelos basados en «patches» binarios de fluidos. 


\section{Capítulo 7}

\section{Conclusiones}

En este trabajo de Tesis se propuso analizar los efectos del flujo inducido sobre las ondas sísmicas en medios parcialmente saturados con distribuciones realistas de fluidos inmiscibles. Con este objetivo, en primer lugar, se presentó una metodología original para calcular la atenuación sísmica de este tipo de medios en el contexto de la teoría de la poroelasticidad de Biot. La misma utiliza la relación entre la potencia disipada y la energía de deformación elástica almacenada en un medio poroelástico deformado por el paso de un campo de ondas sísmicas. Esta técnica permitió validar la hipótesis de medio viscoelástico equivalente, ampliamente utilizada en la literatura, para una serie de escenarios clásicos. Este resultado es de utilidad e importancia, ya que los parámetros viscoelásticos efectivos derivados pueden emplearse en modelos de propagación de ondas sísmicas considerando los efectos disipativos del flujo mesoscópico.

A su vez, la metodología propuesta permitió obtener la contribución local a la atenuación global, una herramienta de análisis que no es posible obtener a través de los métodos convencionales empleados en la literatura. Esta herramienta demostró ser de gran utilidad para explorar las características del proceso difusivo, en particular para comprender el rol que tienen la longitud de difusión asociada, la geometría de las heterogeneidades, y la relación de estas con la respuesta sísmica. Analizando modelos de roca parcialmente saturada con «patches» binarios se observó que la disipación tiene lugar en las zonas saturadas con el fluido mas viscoso que se encuentran en las inmediaciones de las zonas saturadas con el fluido restante. Se observó que, para frecuencias cercanas a la crítica, las regiones donde tiene lugar la mayor parte de la disipación tienden a distribuirse de forma regular alrededor de los «patches» del fluido menos viscoso. Para frecuencias menores, observamos que existe una interacción entre «patches» durante el proceso difusivo, mientras que para frecuencias mayores a la critica la disipación tiende a concentrarse en el entorno inmediato de estos «patches». Al analizar medios fracturados, se observó que la disipación tiene lugar principalmente en la región circundante a los extremos de las fracturas. Cabe destacar que la metodología desarrollada en el Capítulo 3 de este trabajo de Tesis para calcular la contribución local a la atenuación es actualmente considerada una herramienta de gran utilidad para analizar las características internas de los patrones de disipación por flujo inducido, y ha sido utilizada en los trabajos de Rubino et al. (2016), Barbosa et al. (2017), Hunziker et al. (2018) y Barbosa et al. (2018).

Debido a que los campos binarios de saturación no representan adecuadamente las heterogeneidades de mesoescala observadas en el laboratorio, se propuso estudiar la respuesta sísmica de rocas saturadas por campos realistas. Con este fin, se implementó una técnica numérica para resolver las ecuaciones de flujo bifásico que permite obtener la distribución espacial de dos fluidos porales inmiscibles de manera transitoria en 
un medio poroso heterogéneo. Esta técnica fue empleada para modelar un proceso de imbibición considerando la inyección de agua en una roca heterogénea bidimensional inicialmente saturada por gas metano. Se estudiaron las características geométricas de los campos de saturación resultantes y el rol de la tasa de inyección. Para tasas de inyección altas, el proceso de imbibición está dominado por fuerzas viscosas. En este caso, el frente de saturación se presenta como una discontinuidad abrupta dentro de la muestra de roca. A su vez, el agua desplaza el gas existente en la muestra y no se evidencia un significativo entrampamiento de las fases fluidas en forma de regiones aisladas detrás del frente. Por otro lado, al inyectar a tasas bajas el proceso de imbibición está dominado por fuerzas capilares. Entonces, el frente de saturación es irregular y se favorece el entrampamiento en forma de regiones aisladas de agua y gas detrás del frente, asociadas a las heterogeneidades de la matriz. Esto implica que la tasa de inyección determina la distribución espacial de las fases fluidas, y puede elegirse favoreciendo el desplazamiento y/o el entrampamiento de las fases.

Con el objetivo de estudiar el impacto de estas características del campo de saturación en la respuesta sísmica, se emplearon las técnicas previamente descriptas para obtener las curvas de atenuación y dispersión de las ondas sísmicas en la roca de prueba sometida al experimento de imbibición. De esta manera se estimaron los efectos del flujo inducido en las firmas sísmicas. Se observaron dos manifestaciones de flujo inducido, las cuales generan dos máximos de atenuación y dos inflexiones en la curva de dispersión de la velocidad. Las mismas están asociadas a procesos difusivos que tienen lugar debido al contraste de compresibilidad generado por el frente de saturación y a la existencia de «patches» con características continuas detrás del frente de saturación. La primera manifestación ocurre para valores intermedios de saturación global y desaparece cuando el frente de saturación del proceso de imbibición abandona el medio. La segunda manifestación aparece para altos valores de saturación y tiende a aumentar su magnitud con el incremento de la saturación global. Los niveles de atenuación para estas manifestaciones de flujo inducido varían además en función de las características del proceso de imbibición, es decir, si este es impulsado por fuerzas capilares o por fuerzas viscosas. De esta manera, se establece un vínculo directo entre características del proceso de inyección y la respuesta sísmica del medio, lo cual constituye una de las principales contribuciones de este trabajo de Tesis.

Finalmente, se analizaron las consecuencias de representar la respuesta sísmica de medios parcialmente saturados utilizando campos binarios de saturación, ya que estos son frecuentemente utilizados en la literatura. Se consideró una serie de modelos binarios para representar, de manera simple, distribuciones realistas de fluidos inmiscibles. En este sentido, los campos de saturación realistas provienen de realizar modelado numérico de procesos de imbibición en medios homogéneos y heterogéneos. Hemos observado que las estimaciones obtenidas por medio de modelos binarios no pueden reproducir los niveles de atenuación y dispersión, la saturación global, y la frecuencia crítica asociadas al medio realista de forma simultánea. A su vez, empleando la contribución local a la atenuación, observamos que las regiones que contribuyen a disipar la energía por fricción viscosa y las intensidades de la disipación en modelos realistas y binarios son fundamentalmente diferentes. Por todas estas razones, se concluye que los resultados obtenidos en la literatura empleando distribuciones binarias de fluidos deberían reverse o cuestionarse. Asimismo, los resultados de este trabajo de Tesis resal- 
tan la importancia de emplear patrones de saturación realistas, que respeten las leyes de flujo en medios porosos, al modelar los efectos del flujo inducido por ondas sísmicas en medios parcialmente saturados. En este contexto, luego de discutir las limitaciones técnicas y prácticas asociadas al cálculo de los efectos del flujo mesoscópico, se propone un posible flujo de trabajo para analizar los niveles de atenuación y dispersión en medios parcialmente saturados durante procesos de inyección de fluidos.

En síntesis, en el presente trabajo de Tesis se han desarrollado herramientas numéricas y teóricas que permiten mejorar la comprensión actual de la respuesta sísmica de rocas heterogéneas saturadas por fluidos inmiscibles. En particular, es de significativa importancia la vinculación de los procesos de flujo con las características de las curvas de atenuación y dispersión de las ondas sísmicas. La necesidad de emplear campos realistas de saturación se ve resaltada por el hecho de que los modelos binarios de saturación estiman de forma incorrecta la atenuación y la dispersión de las ondas sísmicas por flujo inducido por onda a escalas mesoscópicas. Estos resultados han sido publicados en revistas de la especialidad en los trabajos Solazzi et al. (2014), Solazzi et al. (2016), y Solazzi et al. (2017), y conforman una base teórica sólida para la realización de distintos trabajos a futuro. Entre ellos se destacan explorar los efectos de los fenómenos de histéresis (realizando drenaje e imbibición) en las firmas sísmicas, estudiar los efectos de la relación de viscosidad entre los fluidos inyectados y analizar los efectos del flujo inducido empleando una teoría extendida de Biot que permita considerar dos fluidos inmiscibles en el espacio poral, entre otros. Ampliar la comprensión actual de las conexiones entre los procesos de flujo, la distribución espacial de los fluidos porales, y los observables geofísicos podría permitir alcanzar algunos de los objetivos centrales de la Geofísica Aplicada y Ambiental, tales como la determinación no invasiva de los parámetros hidráulicos de un reservorio, y del tipo de fluido poral y su evolución temporal. 


\section{Agradecimientos}

Estos años de formación han marcado mi vida debido a la interacción constante con profesionales de altísima calidad y a los momentos compartidos con gente maravillosa. Esta tesis no podría haber sido posible sin la guía y la motivación de mis directores, Germán y Luis. Horas de incansables charlas, cuentas, códigos numéricos, y trabajo con Uds. han terminado en forma resumida en estas páginas. Sin embargo, durante estos años se escribió algo mucho más valioso que una tesis doctoral. Gracias por haberme enseñado a pensar de una forma diferente.

La investigación científica es una actividad intensa y puede convertirse, en ciertos casos, en una experiencia altamente frustrante. Gracias a los hermanos que me dio la vida: Gasty, Chino, Flaco, Bruni, Pitu, Mumbru, Leo, Fede, Gusty, y Marian, por no importarles en lo más mínimo los medios porosos y las ondas sísmicas. Se que probablemente nunca lean esta tesis, pero aun así Uds. son mi cable a tierra. También quiero agradecer a Dieguito, Euge, Pabliteno, Nacho, Pani, Facu, Campe, Lau, Mica, Lemele, Nico, Cotita, y Vicky. Citando a Paul Auster (1990): "The story is not in the words, is in the struggle"; gracias por darme ánimo en los momentos de "lucha" y cerveza en los momentos de felicidad.

Quiero agradecerles profundamente a Marina, Damian, Anahí y a Ger por ser mi familia en Suiza. Estudiar en el exterior fue una experiencia extremadamente divertida y enriquecedora, principalmente gracias a su compañía. Son y seguirán siendo una familia para mi. También quiero agradecer a Laureline, Serdar, Alexis, Luma, Titi, Arthur, Cindy, Mathis, Lisa, Ale, Kaltrina, Gabi, y Vale por los momentos que compartimos durante mi estadía en la Lausanne. Por Uds. siempre sentiré a la ciudad de Lausanne como una segunda casa.

Un doctorado constituye un gran desafío intelectual. Tanto en los momentos felices como en los momentos complicados, mi familia ha sido siempre un sinónimo de seguridad, de consejos, y de cariño incondicional. Gracias a mi papá (Omar), a mi mamá (Dina), y a mi hermana (Clara). No existen palabras para expresarles lo fundamentales que son en mi vida. Especial agradecimiento a mi Abuela Hortensia J. Digdanian (Titi), que con sus 91 años es el pilar de esta familia, y a mis tíos (Edith, Horacio, y Daniel), primos (Julia, Cesi, Hora, Ale, y Sabri), y sobrinos (Male, Ade, Tito, Isa, Saru, y Nina). Estoy convencido de que el ruido de la familia en un almuerzo de domingo puede curar cualquier mala sensación que me aqueje. Por ello, todo mi trabajo está dedicado a Uds.

Durante mi doctorado he interactuado con profesionales que ayudaron a enriquecer mis ideas y me han dado consejos invaluables cuando todos los caminos llevaban a un irremediable «segmentation fault». Entre ellos quiero destacar el apoyo de Klaus Holliger, Tobias Müller, y de Marco Milani por sus consejos y excelentes sugerencias. En particular, quiero agradecer a Leonardo Monachesi, que me ha ayudado en momentos claves durante los primeros años de mi doctorado y a quien considero un gran amigo. Más allá de esto, mi alma mater ha sido el departamento de Geofísica Aplicada de la UNLP. No quiero dejar de agradecer a Fabio, Claudia, Danilo, Patricia, Ger, Juani, Peto, Checha, Gelpi y Marian, y a los chicos de "la gemela": Juli, Jony, Fran, y Angie. En particular, quiero dedicarle un especial y robusto agradecimiento a mis amigos Gabo y Sole, a quienes admiro profundamente: Uds. me hacen ser una mejor persona.

La escritura de «papers», la programación de un código numérico, y el ensamblaje de la tesis pueden ser procesos arduos y, a su vez, emocionantes. Durante estas idas y vueltas emocionales, mi eje de referencia fue siempre Lucía. Lula: Gracias por tu paciencia y tu fuerza. Siempre confiaste en mi, me diste cariño, y los más acertados consejos. Sin vos esta tesis no estaría escrita en papel. Gracias por ser mi compañera.

Quiero agradecer brevemente a Alexandra Elbakyan, creadora de sci-hub.com. El acceso conocimiento es una necesidad básica de los seres humanos y posee la potencialidad de 
generar personas más libres y dignas. Su movimiento me permitió acceder gratuitamente al conocimiento científico generado en los más diversos rincones del planeta. Simplemente gracias.

Finalmente quiero agradecer a la Nación Argentina, por financiar mi formación por medio de la UNLP y el CONICET. También quiero agradecer al gobierno Suizo, por financiar mis investigaciones por medio del FCS y la UNIL. Si los límites de nuestro lenguaje son los límites de nuestro mundo ${ }^{1}$, las políticas públicas promovidas por estas entidades gubernamentales me han permitido crecer como individuo y como profesional, nutriéndome de experiencias invaluables. Espero poder devolver con docencia e investigación lo que se me ha dado en formación.

Santiago G. Solazzi

\footnotetext{
1"The limits of my language are the limits of my mind. All I know is what I have words for." (Wittgenstein, 1980).
} 


\section{Bibliografía}

Aki, K. y Richards, P. G. (1980). Quantitative Seismology: Theory and Methods. W. H. Freeman, New York, USA.

Alemu, B. L., Aker, E., Soldal, M., Johnsen, Ø., y Aagaard, P. (2013). Effect of sub-core scale heterogeneities on acoustic and electrical properties of a reservoir rock: $\mathrm{A} \mathrm{CO}_{2}$ flooding experiment of brine saturated sandstone in a computed tomography scanner. Geophys. Prospect., 61:235-250.

Armstrong, R. T., Georgiadis, A., Ott, H., Klemin, D., y Berg, S. (2014). Critical capillary number: Desaturation studied with fast X-ray computed microtomography. Geophys. Res. Lett., 41(1):55-60.

Auster, P. (1990). The New York Trilogy. Penguin.

Barbosa, N. D., Rubino, J. G., Caspari, E., y Holliger, K. (2017). Sensitivity of seismic attenuation and phase velocity to intrinsic background anisotropy in fractured porous rocks: A numerical study. J. Geophys. Res., 122(10):8181-8199.

Barbosa, N. D., Rubino, J. G., Caspari, E., y Holliger, K. (2018). Impact of fracture clustering on the seismic signatures of porous rocks containing aligned fractures. En revisión en: Geophysics.

Baron, L. y Holliger, K. (2011). Constraints on the permeability structure of alluvial aquifers from the poro-elastic inversion of multifrequency P-wave sonic velocity logs. IEEE Trans. Geosci. Remote Sens., 49(6):1937-1948.

Batzle, M. y Wang, Z. (1992). Seismic properties of pore fluids. Geophysics, 57(11):1396-1408.

Batzle, M. L., Han, D., y Hofmann, R. (2006). Fluid mobility and frequency-dependent seismic velocity - direct measurements. Geophysics, 71(1):N1-N9.

Bear, J. (1972). Dynamics of Fluids in Porous Media. Am. Elsevier, New York.

Binning, P. y Celia, M. A. (1999). Practical implementation of the fractional flow approach to multi-phase flow simulation. Adv. Water Resour, 22(5):461-478.

Biot, M. A. (1941). General theory of three-dimensional consolidation. J. Appl. Phys., 12:155-164.

Biot, M. A. (1956a). Theory of propagation of elastic waves in a fluid saturated porous solid. I. Low frequency range. J. Acoust. Soc. Am., 28:168-178.

Biot, M. A. (1956b). Theory of propagation of elastic waves in a fluid saturated porous solid. II. Higher frequency range. J. Acoust. Soc. Am., 28:179-191.

Biot, M. A. (1962). Mechanics of deformation and acoustic propagation in porous media. $J$. Appl. Phys., 33(4):1482-1498. 
Biot, M. A. y Willis, D. G. (1957). The elastic coefficients of the theory of consolidation. J. appl. Mech, 24:594-601.

Borcherdt, R. D. (2009). Viscoelastic Waves in Layered Media. Cambridge University Press.

Bourbié, T., Coussy, O., y Zinszner, B. (1987). Acoustics of porous media. Institut français du pétrole publications, Paris, France.

Brajanovsky, M., Gurevich, B., y Schoenberg, M. (2005). A model for P-wave attenuation and dispersion in a porous medium permeated by aligned fractures. Geophys. J. Int., $163: 372-384$.

British Petroleum Co., B. (2016). Statistical Review of World Energy, June 2016. London: British Petroleum Co.

Brooks, R. y Corey, A. (1964). Hydraulic Properties of Porous Media. Hydrology Papers No. 3. Colorado State University.

Buckley, S. E. y Leverett, M. (1942). Mechanism of fluid displacement in sands. Trans AIME, 146 (01):107-116.

Burdine, N. (1953). Relative permeability calculations from pore size distribution data. $J$. Petrol. Tech., 5(03):71-78.

Cadoret, T., Marion, D., y Zinszner, B. (1995). Influence of frequency and fluid distribution on elastic wave velocities in partially saturated limestones. J. Geophys. Res., 100(B6):97899803 .

Cadoret, T., Mavko, G., y Zinszner, B. (1998). Fluid distribution effect on sonic attenuation in partially saturated limestones. Geophysics, 63:154-160.

Carcione, J. (2007). Wave fields in real media: Wave propagation in anisotropic, anelastic, porous and electromagnetic media. Elsevier, Amsterdam.

Carcione, J. M. y Picotti, S. (2006). P-wave seismic attenuation by slow-wave diffusion: Effects of inhomogeneous rock properties. Geophysics, 71:O1-O8.

Carman, P. (1937). Fluid flow through a granular bed. Trans. Inst. Chem. Eng., 15:150-167.

Caspari, E., Müller, T. M., y Gurevich, B. (2011). Time-lapse sonic logs reveal patchy $\mathrm{CO}_{2}$ saturation in-situ. Geophys. Res. Lett., 38(13).

Celia, M. A. y Binning, P. (1992). A mass conservative numerical solution for two-phase flow in porous media with application to unsaturated flow. Water Resour. Res., 28(10):28192828.

Chapman, M., Liu, E., y Li, X.-Y. (2006). The influence of fluid sensitive dispersion and attenuation on avo analysis. Geophys. J. Int., 167(1):89-105.

Chapman, M., Zatsepin, S. V., y Crampin, S. (2002). Derivation of a microstructural poroelastic model. Geophys. J. Int., 151(2):427-451.

Chapman, S., Tisato, N., Quintal, B., y Holliger, K. (2016). Seismic attenuation in partially saturated Berea sandstone submitted to a range of confining pressures. J. Geophys. Res., 121(3):1664-1676. 
Chavent, G. (1976). A new formulation of diphasic incompressible flows in porous media. En Applications of methods of functional analysis to problems in mechanics, pp. 258-270. Springer.

Chavent, G., Cohen, G., Jaffre, J., Eyard, R., Guerillot, D. R., y Weill, L. (1990). Discontinuous and mixed finite elements for two-phase incompressible flow. SPE Reserv. Eng., 5(04):567-575.

Chavent, G. y Jaffré, J. (1986). Mathematical models and finite elements for reservoir simulation: single phase, multiphase and multicomponent flows through porous media, volumen 17. Elsevier.

Chen, Z., Huan, G., y Ma, Y. (2006). Computational methods for multiphase flows in porous media. SIAM.

Cheng, A. H.-D. (2016). Poroelasticity, volumen 27. Springer.

Conte, S. D. y De Boor, C. (1980). Elementary numerical analysis: An algorithmic approach, volumen 3. McGraw-Hill.

Corey, A. T. (1954). The interrelation between gas and oil relative permeabilities. Producers Monthly, 19(1):38-41.

Courant, R., Friedrichs, K., y Lewy, H. (1928). Über die partiellen Differenzengleichungen der mathematischen Physik. Math. Ann., 100(1):32-74.

Domenico, S. (1974). Effect of water saturation on seismic reflectivity of sand reservoirs encased in shale. Geophysics, 39(6):759-769.

Dubey, R. K. (2010). A composite semi-conservative scheme for hyperbolic conservation laws. App. Math. Comp., 215(9):3335-3342.

Dutta, N. C. y Odé, H. (1979a). Attenuation and dispersion of compressional waves in fluidfilled rocks with partial gas saturation (White model)-Part I: Biot theory. Geophysics, 44:1777-1788, doi:10.1190/1.1440938.

Dutta, N. C. y Odé, H. (1979b). Attenuation and dispersion of compressional waves in fluid-filled rocks with partial gas saturation (White model)-Part II: Results. Geophysics, 44:1789-1805.

Dvorkin, J., Mavko, G., y Nur, A. (1995). Squirt flow in fully saturated rocks. Geophysics, 60(1):97-107.

Dvorkin, J., Nolen-Hoeksema, R., y Nur, A. (1994). The squirt-flow mechanism: Macroscopic description. Geophysics, 59(3):428-438.

Frenkel, J. (1944). On the theory of seismic and seismo-electric phenomena in moist soils. $J$. Phys, 8:230-241.

Fučík, R. y Mikyška, J. (2011). Discontinous galerkin and mixed-hybrid finite element approach to two-phase flow in heterogeneous porous media with different capillary pressures. Procedia Comput. Sci., 4:908-917. 
Gassmann, F. (1951). Über die elastizität poröser medien. Vierteljahrsschrift der Naturforschenden Gesellschaft in Zurich, 96:1-23.

Georgiadis, A., Berg, S., Makurat, A., Maitland, G., y Ott, H. (2013). Pore-scale microcomputed-tomography imaging: Nonwetting-phase cluster-size distribution during drainage and imbibition. Phys. Rev. E, 88(3):033002.

Goldstein, H.and Poole, C. P. y Safko, J. L. (2014). Classical Mechanics: Pearson New International Edition. Pearson Higher Ed.

Green, E. I. (1955). The story of Q. Am. Sci., 43(4):584-594.

Guarracino, L. y Santos, J. E. (2004). Stochastic modeling of variably saturated transient flow in fractal porous media. Math. Geology, 36(2):217-238.

Gurevich, B., Brajanovsky, M., Galvin, R. J., Müller, T. M., y Toms-Stewart, J. (2009). P-wave dispersion and attenuation in fractured and porous reservoirs- poroelasticity approach. Geophys. Prospect., 57:225-237.

Gurevich, B. y Lopatnikov, S. L. (1995). Velocity and attenuation of elastic waves in finely layered porous rocks. Geophysics, 121:933-947.

Hashin, Z. (1964). Theory of mechanical behavior of heterogeneous media. Appl. Mech. Rev., $17: 1-9$.

Helmig, R., Miller, C. T., Jakibs, H., Class, H., Hilpert, M., Kees, C. E., y Niessner, J. (2006). Multiphase flow and transport modeling in heterogeneous porous media. En Di Bucchianico, A., Mattheij, R., y Peletier, M., editores, Progress in Industrial Mathematics at ECMI 2004, pp. 449-488. Springer.

Hilfer, R. y Øren, P. E. (1996). Dimensional analysis of pore scale and field scale immiscible displacement. Transp. Porous Med., 22(1):53-72.

Hill, R. (1963). Elastic properties of reinforced solids: Some theoretical principles. J. Mech. Phys. Solids, 11:357-372.

Hill, R. (1972). On constitutive maco-variables for heterogeneous solids at finite strain. Proc. R. Soc. Lond. A, 326:131-147.

Hoteit, H. y Firoozabadi, A. (2008). Numerical modeling of two-phase flow in heterogenous permeable media with different capillary pressures. Adv. Water Resour., 31:56-73.

Hunziker, J., Favino, M., Caspari, E., Quintal, B., Rubino, J. G., Krause, R., y Holliger, K. (2018). Seismic attenuation and stiffness modulus dispersion in porous rocks containing stochastic fracture networks. J. Geophys. Res.

Iglauer, S., Paluszny, A., Pentland, C. H., y Blunt, M. J. (2011). Residual $\mathrm{CO}_{2}$ imaged with X-ray micro-tomography. Geophys. Res. Lett., 38(21).

Jänicke, R., Quintal, B., y Steeb, H. (2015). Numerical homogenization of mesoscopic loss in poroelastic media. Eur. J. Mech. A Solids, 49:382-395.

Johnson, D. L. (2001). Theory of frequency dependent acoustics in patchy-saturated porous media. J. Acoust. Soc. Am., 110:682-694. 
Johnson, D. L., Koplik, J., y Dashen, R. (1987). Theory of dynamic permeability and tortuosity in fluid-saturated porous media. J. Fluid Mech., 176:379-402.

Johnston, D. H., Toksöz, M. N., y Timur, A. (1979). Attenuation of seismic waves in dry and saturated rocks: II. Mechanisms. Geophysics, 44(4):691-711.

Knight, R. y Nolen-Hoeksema, R. (1990). A laboratory study of the dependence of elastic wave velocities on pore scale fluid distribution. Geophys. Res. Lett., 17(10):1529-1532.

Kou, J. y Sun, S. (2010). A new treatment of capillarity to improve the stability of impes two-phase flow formulation. Comput. Fluids, 39(10):1923-1931.

Kozeny, J. (1927). Über kapillare leitung des wassers im boden. Wien, Akad. Wiss., 136(2a):271-306.

Krause, M., Krevor, S., y Benson, S. M. (2013). A procedure for the accurate determination of sub-core scale permeability distributions with error quantification. Transport Porous Med., 98(3):565-588.

Krause, M. H., Perrin, J. C., y Benson, S. M. (2009). Modeling permeability distributions in a sandstone core for history matching coreflood experiments. SPE Journal, 16(04):768-777.

Krevor, S., Pini, R., Li, B., y Benson, S. M. (2011). Capillary heterogeneity trapping of $\mathrm{CO}_{2}$ in a sandstone rock at reservoir conditions. Geophys Res. Lett., 38(15).

Kuteynikova, M., Tisato, N., Jänicke, R., y Quintal, B. (2014). Numerical modeling and laboratory measurements of seismic attenuation in partially saturated rock. Geophysics, 79:L13-L20.

Lawyer, L. C., Bates, C. C., y Rice, R. B. (2001). Geophysics in the affairs of mankind: A personalized history of exploration geophysics. Society of Exploration Geophysicists.

Le Ravalec, M., Guéguen, Y., y Chelidze, T. (1996). Elastic wave velocities in partially saturated rocks: Saturation hysteresis. J. Geophys. Res., 101:837-844.

Lebedev, M., Iglauer, S., y Mikhaltsevich, V. (2014). Acoustic response of reservoir sandstones during injection of supercritical $\mathrm{CO}_{2}$. Energy Proc., 63:4281-4288.

Lebedev, M., Toms-Stewart, J., Clennell, B., Peruvukhina, M., Shulakova, V., Paterson, L., Müller, T. M., Gurevich, B., y Wenzlau, F. (2009). Direct laboratory observation of patchy saturation and its effects on ultrasonic velocities. The Leading Edge, 28(1):24-27.

Lenormand, R. (1990). Liquids in porous media. J. Phys. Condens. Matter, 2:79-88.

Lenormand, R. y Zarcone, C. (1984). Role of roughness and edges during imbibition in square capillaries. En SPE Annual Technical Conference and Exhibition. Society of Petroleum Engineers.

Leverett, M. (1941). Capillary behavior in porous solids. Trans. AIME Petr. Eng. Div., $69: 142-152$.

Li, B. y Benson, S. M. (2015). Influence of small-scale heterogeneity on upward $\mathrm{CO}_{2}$ plume migration in storage aquifers. Adv. Water Resour., 83:389-404. 
Liu, J., Müller, T. M., Qi, Q., Lebedev, M., y Sun, W. (2016). Velocity-saturation relation in partially saturated rocks: Modelling the effect of injection rate changes. Geophys. Prospect., 64(4):1054-1066.

Lopes, S. y Lebedev, M. (2012). Research note: Laboratory study of the influence of changing the injection rate on the geometry of the fluid front and on $\mathrm{P}$-wave ultrasonic velocities in sandstone. Geophys. Prospect., 28(1):24-27.

Lopes, S., Lebedev, M., Müller, T. M., Clennell, M. B., y Gurevich, B. (2014). Forced imbibition into a limestone: Measuring P-wave velocity and water saturation dependence on injection rate. Geophys. Prospect., 62(5):1126-1142.

Mair, R. W., Wong, G. P., Hoffmann, D., Hürlimann, M. D., Patz, S., Schuartz, L. M., y Walsworth, R. L. (1999). Probing porous media with gas diffusion NMR. Phys. Rev. lett., 83(16):3324-3327.

Margat, J. y Van der Gun, J. (2013). Groundwater around the world: a geographic synopsis. CRC Press.

Masson, T. J. y Pride, S. R. (2007). Poroelastic finite difference modeling of seismic attenuation and dispersion due to mesoscopic-scale heterogeneity. J. Geophys. Res., 112, B03204.

Masson, Y. J. y Pride, S. R. (2011). Seismic attenuation due to patchy saturation. J. Geophys. Res., 116:B0326.

Masson, Y. J. y Pride, S. R. (2014). On the correlation between material structure and seismic attenuation anisotropy in porous media. J. Geophys. Res. Solid Earth, 119, B03206.

Mavko, G., Mukerji, T., y Dvorkin, J. (2009). The Rock Physics Handbook: Tools for Seismic Analysis of Porous Media. Cambridge University Press.

Mavko, G. M. y Nur, A. (1979). Wave attenuation in partially saturated rocks. Geophysics, 44:161-178, doi:10.1190/1.1440958.

Mikhaltsevitch, V., Lebedev, M., y Gurevich, B. (2016). Laboratory measurements of the effect of fluid saturation on elastic properties of carbonates at seismic frequencies. Geophys. Prospect., 64(4):799-809.

Milani, M., Rubino, J. G., Baron, L., Sidler, R., y Holliger, K. (2015). Attenuation of sonic waves in water-saturated alluvial sediments due to wave-induced fluid flow at microscopic, mesoscopic and macroscopic scales. Geophys J. Int., 203(1):146-157.

Milani, M., Rubino, J. G., Müller, T. M., Quintal, B., Caspari, E., y Holliger, K. (2016). Representative elementary volumes for evaluating effective seismic properties of heterogeneous poroelastic media. Geophysics, 81:D21-D33.

Monsen, K. y Johnstad, S. E. (2005). Improved understanding of velocity-saturation relationships using 4D computer-tomography acoustic measurements. Geophys. Prospect., 53(2):173-181.

Mualem, Y. (1976). A new model for predicting the hydraulic conductivity of unsaturated porous media. Water Resour. Res., 12(3):513-522. 
Müller, T. M., Gurevich, B., y Lebedev, M. (2010). Seismic wave attenuation and dispersion resulting from wave-induced flow in porous rocks - a review. Geophysics, 75:147-163.

Müller, T. M. y Rothert, E. (2006). Seismic attenuation due to wave-induced flow: Why Q in random structures scales differently. Geophys. Res. Lett., 33:doi:10.1029/2006GL026789.

Murphy, W. (1984). Acoustic measures of partial gas saturation in tight sandstones. $J$. Geophys. Res., 89:11,549-11,559.

Nakagawa, S., Kneafsey, T. J., Daley, T. M., Freifeld, B. M., y Rees, E. V. (2013). Laboratory seismic monitoring of supercritical $\mathrm{CO}_{2}$ flooding in sandstone cores using the split hopkinson resonant bar technique with concurrent X-ray computed tomography imaging. Geophys. Prospect., 61(2):254-269.

Nakagawa, S. y Schoenberg, A. (2007). Poroelastic modeling of seismic boundary conditions across a fracture. J. Acoust. Soc. Am., 122:831-847.

Nedelec, J. C. (1980). Mixed finite elements in $\mathrm{r}^{3}$. Numer. Math., 35:315-341.

Norris, A. N. (1993). Low-frequency dispersion and attenuation in partially saturated rocks. J. Acoust. Soc. Am., 94:359-370.

O'Connell, R. J. y Budiansky, B. (1978). Measures of dissipation in viscoelastic media. Geophys. Res. Lett., 5:5-8.

Pavuluri, S. (2014). Kinetic Approach for Modeling Salt Precipitation in Porous-Media. GRIN Publishing.

Peaceman, D. W. (1977). Fundamentals of Numerical Reservoir Simulation. Elsevier, New York.

Perrin, J.-C. y Benson, S. (2010). An experimental study on the influence of sub-core scale heterogeneities on $\mathrm{CO}_{2}$ distribution in reservoir rocks. Trans. porous med., 82(1):93-109.

Picotti, S., Carcione, J. M., Rubino, J. G., Santos, J. E., y Cavallini, F. (2010). A viscoelastic representation of wave attenuation in porous media. Comput. Geosci., 36:44-53.

Pini, R. y Benson, S. M. (2013). Characterization and scaling of mesoscale heterogeneities in sandstones. Geophys. Res. Lett., 40(15):3903-3908.

Pini, R. y Benson, S. M. (2017). Capillary pressure heterogeneity and hysteresis for the supercritical $\mathrm{CO}_{2} /$ water system in a sandstone. Adv. Water Resour.

Pini, R., Krevor, S. C., y Benson, S. M. (2012). Capillary pressure and heterogeneity for the $\mathrm{CO}_{2}$ /water system in sandstone rocks at reservoir conditions. Adv. Water Resour., $38: 48-59$.

Plona, T. J. y Johnson, R. K. (1980). Biot's second compressional wave: measurement and theory. J. Acoust. Soc. Am., 68(S1):S14-S14.

Pride, S. R. (2005). Relationships between seismic and hydrological properties. En Rubin, Y. y Hubbard, S., editores, Hydrogeophysics, capítulo 9, pp. 253-290. Springer.

Pride, S. R. y Berryman, J. G. (2003). Linear dynamics of double-porosity dual-permeability materials. I. Governing equations and acoustic attenuation. Phys. Rev. E, 68, 036603. 
Pride, S. R., Berryman, J. G., y Harris, J. M. (2004). Seismic attenuation due to wave-induced flow. J. Geophys. Res., 109, B01201.

Pride, S. R., Tromeur, E., y Berryman, J. G. (2002). Biot slow-wave effects in stratified rock. Geophysics, 67(1):271-281.

Qi, Q., Müller, T. M., Gurevich, B., Lopes, S., Lebedev, M., y Caspari, E. (2014a). Quantifying the effect of capillarity on attenuation and dispersion in patchy-saturated rocks. Geophysics, 79:WB35-WB50.

Qi, Q., Müller, T. M., y Rubino, J. G. (2014b). Seismic attenuation: Effects of interfacial impedance on wave-induced pressure difusion. Geophys. J. Int.

Quintal, B., Schmalholz, S. M., y Podladchikov, Y. (2008). Low-frequency reflections from a thin layer with high attenuation caused by interlayer flow. Geophysics, 74(1):N15-N23.

Quintal, B., Steeb, H., Frenher, M., y Schmalholz, S. M. (2011). Quasi-static finite element modeling of seismic attenuation and dispersion due to wave-induced fluid flow in poroelastic media. J. Geophys. Res., 116:B01201.

Rahman, T., Lebedev, M., Barifcani, A., e Iglauer, S. (2016). Residual trapping of supercritical $\mathrm{CO}_{2}$ in oil-wet sandstone. J. Colloid Interface Sci., 469:63-68.

Raviart, P. y Thomas, J. (1977). Mixed finite element method for 2nd order elliptic problems. Lect. Notes Math., 606:292-315.

Ren, H., Goloshubin, G., y Hilterman, F. J. (2009). Poroelastic analysis of amplitude-versusfrequency variations. Geophysics, 74(6):N41-N48.

Ren, Q. Y., Chen, G. J., Yan, W., y Guo, T. M. (2000). Interfacial tension of $\left(\mathrm{CO}_{2}+\right.$ $\left.\mathrm{CH}_{4}\right)+$ water from $298 \mathrm{~K}$ to $373 \mathrm{~K}$ and pressures up to $30 \mathrm{MPa}$. J. Chem. Eng. Data, 45(4):610-612.

Renaud, G., Rivière, J., Le Bas, P.-Y., y Johnson, P. A. (2013). Hysteretic nonlinear elasticity of Berea sandstone at low-vibrational strain revealed by dynamic acousto-elastic testing. Geophys. Res. Lett., 40:715-719.

Riaz, A., Tang, G. Q., Tchelepi, H. A., y Kovscek, A. R. (2007). Forced imbibition in natural porous media: Comparison between experiments and continuum models. Phys. Rev. E, $75(3), 036305$.

Rubino, J. g., Caspari, E., Müller, T. M., Milani, M., Barbosa, N. D., y Holliger, K. (2016). Numerical upscaling in 2-d heterogeneous poroelastic rocks: Anisotropic attenuation and dispersion of seismic waves. J. Geophys. Res., 121(9):6698-6721.

Rubino, J. G., Guarracino, L., Müller, T. M., y Holliger, K. (2013a). Do seismic waves sense fracture connectivity? Geophys. Res. Lett., 40(4):692-696.

Rubino, J. G. y Holliger, K. (2012). Seismic attenuation and velocity despersion in heterogeneous partially saturated porous rocks. Geophys. J. Int., 188:1088-1102.

Rubino, J. G., Monachesi, L. B., Müller, T. M., Guarracino, L., y Holliger, K. (2013b). Seismic wave attenuation and dispersion due to wave-induced fluid flow in rocks with strong permeability fluctuations. J. Acoust. Soc. Am., pp. 4742-4751. 
Rubino, J. G., Müller, T. M., Guarracino, L., Milani, M., y Holliger, K. (2014). Seismoacustic signatures of fracture connectivity. J. Geophys. Res., 119:2252-2271.

Rubino, J. G., Ravazzoli, C. L., y Santos, J. E. (2006). Reflection and transmission of waves in composite porous media: A quantification of energy conversions involving slow waves. J. Acoust. Soc. Am., 120 (5):2425-2436.

Rubino, J. G., Ravazzoli, C. L., y Santos, J. E. (2009). Equivalent viscoelastic solids for heterogeneous fluid-saturated porous rocks. Geophysics, 74:N1-N13.

Rubino, J. G. y Velis, D. R. (2011). Seismic characterization of thin beds containing patchy carbon dioxide-brine distributions: A study based on numerical simulations. Geophysics, 76(3):R57-R67.

Rubino, J. G., Velis, D. R., y Sacchi, M. D. (2011). Numerical analysis of wave-induced fluid flow effects on seismic data: Application to monitoring of $\mathrm{CO}_{2}$ storage at the Sleipner field. J. Geophys. Res., 116:1088-1102.

Sams, M. S., Neep, J. P., Worthington, M. H., y King, M. S. (1997). The measurement of velocity dispersion and frequency-dependent intrinsic attenuation in sedimentary rocks. Geophysics, 62(5):1456-1464.

Sayar, P. y Torres-Verdín, C. (2017). Effective medium modeling of velocity dispersion and attenuation in isotropic rocks. Geophysics, 82(2):D135-D156.

Shi, J., Xuw, Z., y Durucan, S. (2011). Supercritical $\mathrm{CO}_{2}$ core flooding and imbibition in Tako sandstone- Influence of sub-core scale heterogeneity. Int. J. Greenh. Gas Con., 5:75-87.

Sidler, R., Rubino, J. G., y Holliger, K. (2013). Quantitative comparison between simulations of seismic wave propagation in heterogeneous poro-elastic media and equivalent viscoelastic solids for marine-type environments. Geophys. J. Int., 193:463-474.

Solazzi, S. G., Guarracino, L., Rubino, J. G., Müller, T. M., y Holliger, K. (2017). Modeling forced imbibition processes and the associated seismic attenuation in heterogeneous porous rocks. J. Geophys. Res., 122(11):9031-9049.

Solazzi, S. G., Rubino, J. G., Müller, T. M., Milani, M., Guarracino, L., y Holliger, K. (2014). An energy-based approach to estimate seismic attenuation due to wave-induced fluid flow. En SEG Technical Program Expanded Abstracts 2014, pp. 1991-1995.

Solazzi, S. G., Rubino, J. G., Müller, T. M., Milani, M., Guarracino, L., y Holliger, K. (2016). An energy-based approach to estimate seismic attenuation due to wave-induced fluid flow in heterogeneous poroelastic media. Geophys. J. Int., 207(2):823-832.

Soldi, M., Guarracino, L., y Jougnot, D. (2017). A simple hysteretic constitutive model for unsaturated flow. Trans. Porous Med., 120(2):271-285.

Spencer, J. W. y Shine, J. (2016). Seismic attenuation and modulus dispersion in sandstones. Geophysics, 81(3):D219-D239.

Teja, A. S. y Rice, P. (1981). Generalized corresponding states method for viscosities of liquid mixtures. Ind. Eng. Chem. Fundam., 20:77-81.

Terzaghi, K. (1943). Theoretical soil mechanics. Wiley. 
Tisato, N. y Madonna, C. (2012). Attenuation at low seismic frequencies in partially saturated rocks: Measurements and description of a new apparatus. J. Appl, Geophys., 86:44 - 53.

Tisato, N. y Quintal, B. (2013). Measurements of seismic attenuation and transient fluid pressure in partially saturated Berea sandstone: Evidence of fluid flow on the mesoscopic scale. Geophys. J. Int., 195:342-351.

Tod, S. R. (2003). An anisotropic fractured poroelastic effective medium theory. Geophys. J. Int., 155(3):1006-1020.

Toms, J., Müller, T. M., y Gurevich, B. (2007). Seismic attenuation in porous rocks with random patchy saturation. Geophys. Prospect., 55(5):671-678.

Toms-Stewart, J., Müller, T. M., Gurevich, B., y Paterson, L. (2009). Statistical characterization of gas-patch distributions in partially saturated rocks. Geophysics, 72(2):WA51-WA64.

Tronicke, J. y Holliger, K. (2005). Quantitative integration of hydrogeophysical data: Conditional geostatistical simulation for characterizing heterogeneous alluvial aquifers. Geophysics, 70:H1-H10.

UNESCO (2003). The World Water Development Report 1: Water for People, Water for Life.

van Genuchten, M. T. (1980). A closed-form equation for predicting the hydraulic conductivity fo unsaturated soils. Soil Sci. Soc. Am. J., 44:892-898.

Vogelaar, B. y Smeulders, D. (2007). Extension of White's layered model to the full frequency range. Geophys. Prospect., 55:685-695.

Wang, H. F. (2017). Theory of linear poroelasticity with applications to geomechanics and hydrogeology. Princeton University Press.

Welge, H. J. (1952). A simplified method for computing oil recovery by gas or water drive. Trans AIME, 195:91-99.

Wenzlau, F., Altman, J., y Müller, T. M. (2010). Anisotropic dispersion and attenuation due to wave-induced fluid flow: Quasi-static finite element modeling in poroelastic solids. $J$. Geophys. Res., 115:B07204.

White, J. E. (1975). Computed seismic speeds and attenuation in rocks with partial gas saturation. Geophysics, 40:224-232.

White, J. E., Mikhaylova, N. G., y Lyakhovitsky, F. M. (1975). Low-frequancy seismic waves in fluid-saturated layered rocks. Phys. Solid Earth, 11:654-659.

Winkler, K. y Nur, A. (1979). Pore fluids and seismic attenuation in rocks. Geophys. Res. Lett., 6.1:1-4.

Winkler, K. W. (1985). Dispersion analysis of velocity and attenuation in Berea sandstone. J. Geophys. Res., 90(B8):6793-6800.

Wittgenstein, L. (1980). Tractatus logico-philosophicus logisch-philosophische abhandlung.

Wood, A. (1955). A Textbook of Sound. MacMillan Publishing Company, New York. 
Yang, H., Yang, C., y Sun, S. (2016). Active-set reduced-space methods with nonlinear elimination for two-phase flow problems in porous media. SIAM J. Sci. Comput., 38(4):B593B618.

Yin, C. S., Batzle, M. L., y Smith, B. J. (1992). Effects of partial liquid/gas saturation on extensional wave attenuation in Berea sandstone. Geophys. Res. Lett., 19(13):1399-1402.

Zhang, Y., Nishizawa, O., Kiyama, T., y Xue, Z. (2015). Saturation-path dependency of $\mathrm{P}$-wave velocity and attenuation in sandstone saturated with $\mathrm{CO}_{2}$ and brine revealed by simultaneous measurements of waveforms and X-ray computed tomography images. Geophysics, 80:D403-D415. 